\title{
Modernisierung und Erweiterung des staatlichen Bildungswesens in Taiwan im Zeitraum von 1885 bis 1987
}

\author{
Dissertation zur Erlangung des Doktorgrades \\ der Sozialwissenschaftlichen Fakultät \\ der Georg-August-Universität Göttingen
}

\author{
Vorgelegt von: \\ Yatzu Chuang \\ aus Kaohsiung, Taiwan
}

Göttingen, Dezember 2011 


\section{Inhaltsverzeichnis}

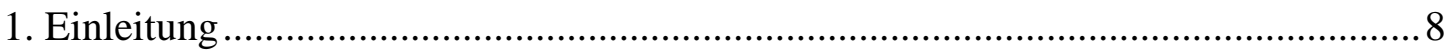

1.1. Fragestellung und Forschungsstand ...............................................................

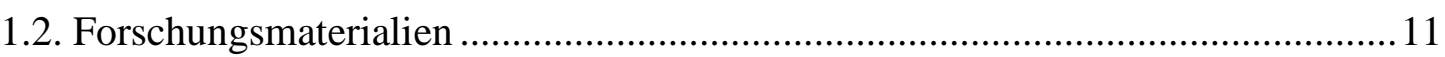

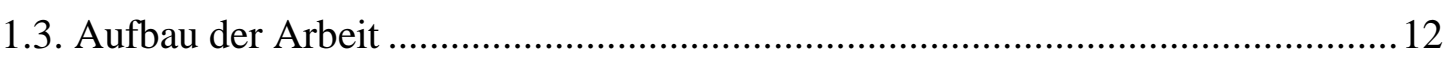

2. Konfuzianische Erziehung und das kaiserliche Beamtenprüfungssystem ................14

2.1. Grundgedanken des Konfuzianismus zur Erziehung ......................................... 14

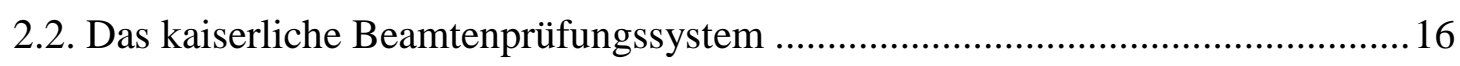

2.2.1. Die Entstehung des kaiserlichen Beamtenprüfungssystems ...............................16

2.2.2. Die Stufung des kaiserlichen Beamtenprüfungssystems .................................... 17

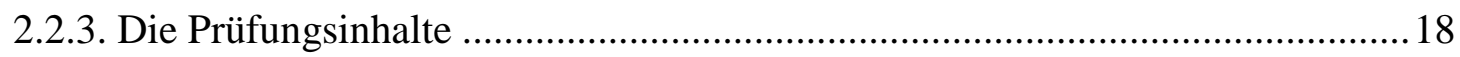

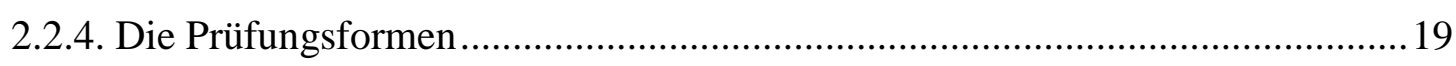

2.2.5. Voraussetzungen für das Teilnehmen an die kaiserliche Beamtenprüfungen ....21

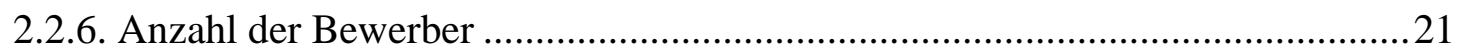

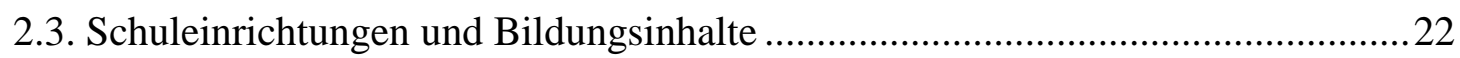

2.3.1. Einrichtungen und Lehrinhalte der elementaren Erziehung und Bildung .........22

2.3.2. Einrichtungen und Lehrinhalte der höheren Bildung.........................................26

2.4. Wirkung des kaiserlichen Beamtenprüfungssystems..........................................28

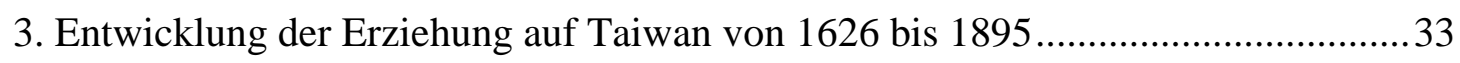

3.1. Erziehung unter der Besatzung der Spanie und Niederland von 1626 bis $1662 \ldots 33$

3.2. Erziehung unter dem Ming- und Ching-Kaiserreich von 1662 bis 1894 auf

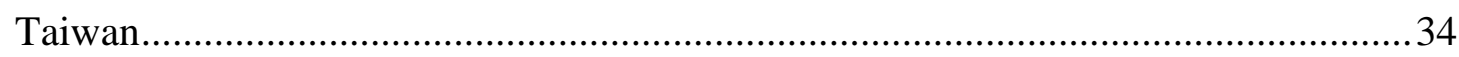

3.2.1. Begann mit der Errichtung der konfuzianischen Bildungseinrichtungen von

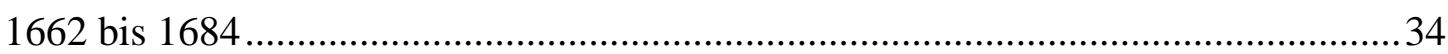

3.2.2. Erweiterung der Erziehungseinrichtungen unter dem Ching-Kaiserreich von

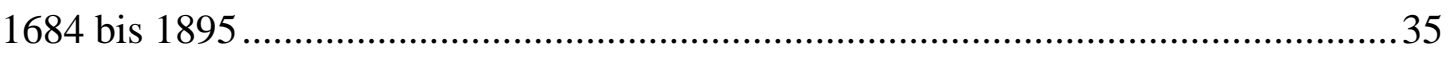

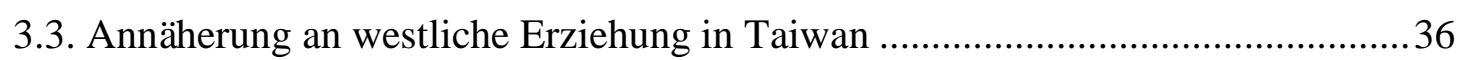

3.3.1. Beginn mit der Einführung der westlichen Bildungsformen innerhalb der „Selbststärkungsbewegung“ im Ching-Kaiserreich von 1862 bis 1894 ........................36

3.3.2. Konstruktion der Provinz Taiwan und Einführung der Modernisierungsprojekte

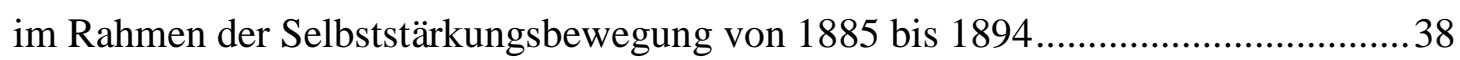
3.3.3. Erster Versuch für die Errichutng der westlichen Bildungsformen von 1885 bis 1894

4. Aufbau des modernen Bildungssystems unter der japanischen Kolonial- herrschaft von 1895 bis 1944 
4.1. Japanische Bildungspolitik im 19. Jahrhundert

4.2. Bildungspolitik unter der japanischen Kolonialherrschaft ..................................43

4.3. Aufbauprozess des modernen Bildungssystems ..............................................47

4.3.1. Die Primarstufe: Elementarbildung und öffentliche Schule .............................47

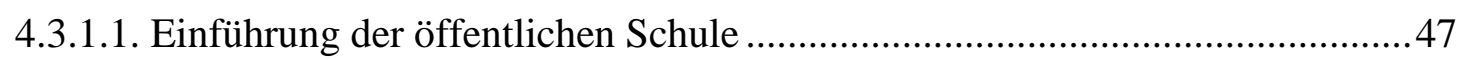

4.3.1.2. Die konfuzianische Schule als Übergang der öffentlichen Schulen ...............49

4.3.1.3. Erweiterung der elementaren Schulen ........................................................51

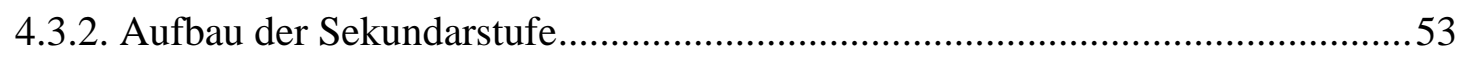

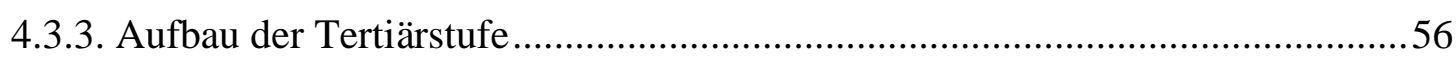

5. Debatte über die Gestaltung eines modernen staatlichen Bildungswesens 1898 bis 1949

5.1. Entwurf eines modernen staatlichen Bildungswesens im Zug der Hundert- tage Reform 1898

5.2. Das erste Edikt zur Gestaltung eines modernen staatlichen Bildungswesens im

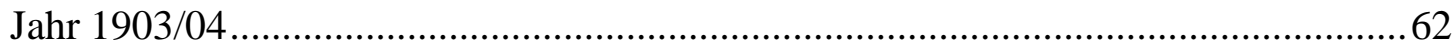

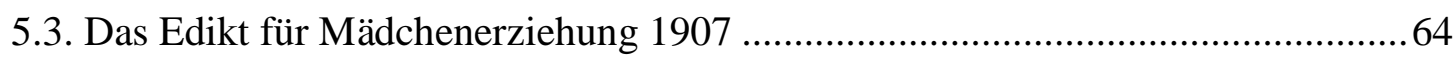

5.4. Bildungsreformen zum Beginn der Grüdnung der Republik China .....................65

5.4.1. Reform des staatlichen Bildungswesens nach der Gründung der Republik 1912

5.4.2. Reform des Bildungswesens nach der „Bewegung des Vierten Mai 1919“......67

5.5. Gestaltung des Bildungswesens unter dem KMT-Regime von 1928 bis 1949 ....72

5.5.1. Suns „Drei Prinzipien des Volkes“ ................................................................... 72

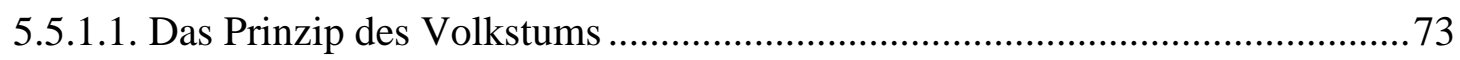

5.5.1.2. Das Prinzip der Volksrechte .................................................................... 75

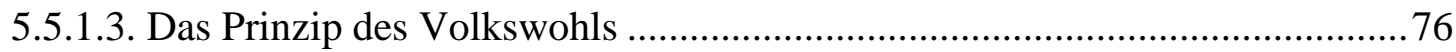

5.5.2. Die „Drei Prinzipien des Volkes“ als Grundlage des Bildungswesens ..............80

6. Umsetzung der Bildungspolitik gemäß den „Drei Prinzipien des Volks“ in Taiwan

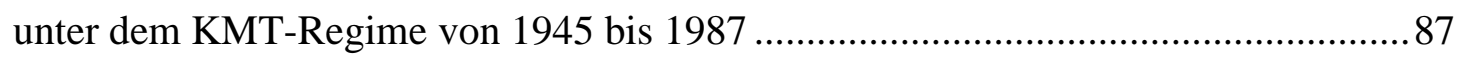

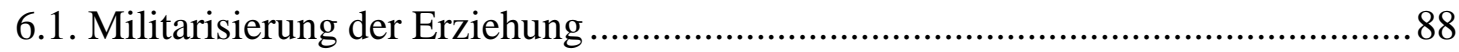

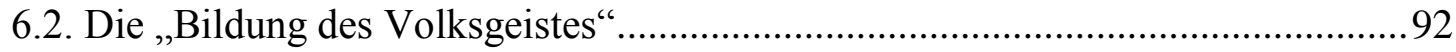

6.2.1. Die Vereinheitlichung der Verkehrssprache ....................................................93

6.2.2. Bewegung der Dejapanisierung bzw. Sinisierung ..........................................96

6.2.3. Bewegung zur Verbreitung der Nationalsprache ...........................................97

6.2.4. Bewegung für die Bewahrung und Restauration der chinesischen Kultur ........98

6.2.4.1. Historischer Hintergrund der „Bewegung für die Bewahrung und Restauration

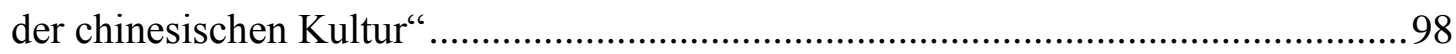

6.2.4.2. Chiangs Forderung nach „Ethik, Demokratie und Wissenschaft"“............... 104

6.2.4.3. Inhalte der „Bewegung für die Bewahrung und Restauration der chinesischen 
Kultur"

6.2.4.4. Der Einfluss der „Bewegung für die Bewahrung und Restauration der chinesischen Kultur" auf die schulische Erziehung....

6.3. Pläne für die Gestaltung der beruflichen Ausbildung sowie der naturwissenschaftlichen und technischen Forschung und Bildung

6.3.1. Die Debatte über berufliche Ausbildung sowie naturwissenschaftliche und technische Forschung und Bildung innerhalb des KMT .......................................... 115

6.3.2. Unterstützung durch die US-Entwicklungshilfe .......................................... 120

6.3.3. Das Gutachten US-amerikanischer Berater ...................................................... 123

6.3.4. Aufbau der beruflichen Ausbildung............................................................. 125

6.3.4.1. Aufbau der Lehrerausbildung und Umorganisation der Fachhochschulen... 126

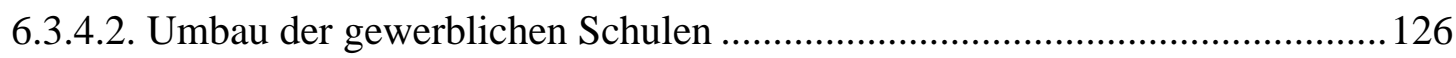

6.3.4.3. Aufbau landwirtschaftlicher Berufsschulen............................................... 127

6.3.5. Aufbau naturwissenschaftlicher und technischer Forschung und Aus bildung 129

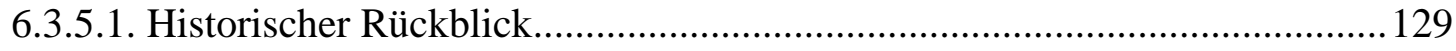

6.3.5.2. Die taiwanesisch-amerikanische Kooperation ............................................ 131

6.3.5.3. Regimekritik infolge der wissenschaftlichen Kooperation........................... 137

6.3.5.4. Der „Direct Council for Development of Science“ ....................................... 140

7. Der Umbau des Bildungssystems von 1945 bis 1987 ........................................... 143

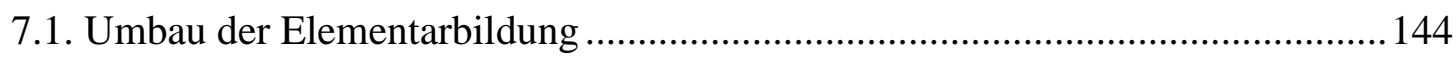

7.2. Umbau des Bildungswesens: sekundäre und tertiäre Stufe ............................... 144

7.3. Der Erweiterungsprozess des Bildungssystems von 1945 bis 1987 .................. 145

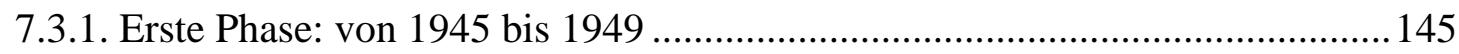

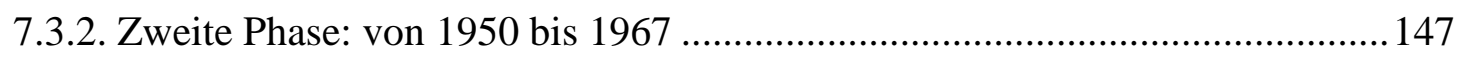

7.3.2.1. Erweiterung der Elementar- und Sekundarstufe ........................................ 148

7.3.2.2. Erweiterung differenzierter Ausbildungsgänge .......................................... 150

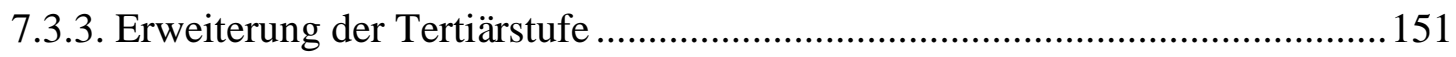

7.3.3.1. Ausbau von Universitäten und Fachhochschulen ........................................ 151

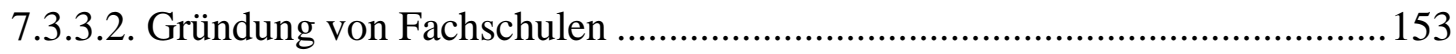

7.3.3.3. Schaffung von Abendkursen und Förderkursen ........................................ 153

7.4. Die Konzepte „Human Capital“ und „Human Resources“ “.................................. 154

7.5. Bildungsökonomie und staatliche Planung ....................................................... 157

7.5.1. Der Forschungsbericht des „Stanford Research Institute“.............................. 158

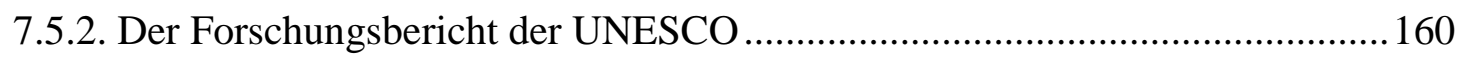

7.5.3. Die „Arbeitsgruppe Arbeitskräfteplanung“ .................................................... 162

7.5.4. Die „Pläne zur Ausbildung von Arbeitskräften“ “............................................. 166

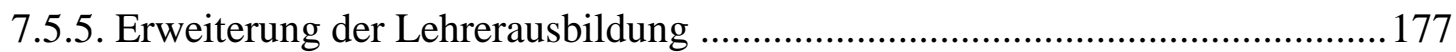

7.5.6.Ungleiche staatliche Unterstützung der allgemeinbildenden und beruflichen 
Bildungszweige.

8. Das einheitliche Aufnahmeprüfungssystem als Methode für Verteilung der

Bildungsplätze.

8.1. Einführung der Abschlussprüfung und des einheitlichen

Aufnahmeprüfungssystems zur Hochschule auf dem chinesischen Festland in den 1930er Jahren

8.2. Einführung eines einheitlichen Aufnahmeprüfungssystems in Taiwan.............. 183

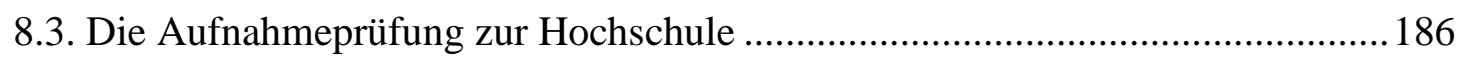

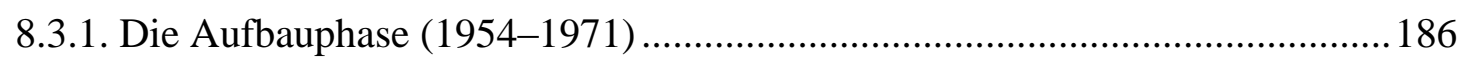

8.3.1.1. Die Organisation des Aufnahmeprüfungssystems ....................................... 186

8.3.1.2. Voraussetzungen für die Teilnahme an der Aufnahmeprüfung .................... 187

8.3.1.3. Gliederung der Studienfächer und Gruppierung der Prüfungsteilnehmer .... 188

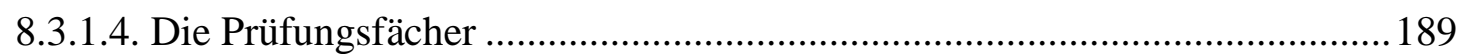

8.3.1.5. Prüfungsaufgaben und -verfahren......................................................... 192

8.3.1.6.Berechnungsmethoden der Prüfungsergebnisse und Verteilungsverfahren .. 193

8.3.1.7. Sondermaßnahmen für bestimmte soziale Gruppen ................................... 194

8.3.2. Die Erneuerungsphase (1972-1986) ............................................................. 195

8.3.2.1. Umorganisation des Aufnahmeprüfungssystems ........................................ 195

8.3.2.2. Änderung von Anmeldung zur Prüfung und Zuteilung der Studienplätze ... 198

8.3.2.3. Neue Aufgabenstellung und Neubewertung der Prüfungsergebnisse............ 199

9. Der Kampf um einen Studienplatz.........................................................................201

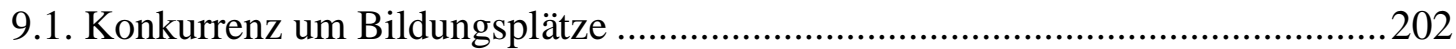

9.1.1. Qualitative Abstufungen bei gleicher Schulform ........................................202

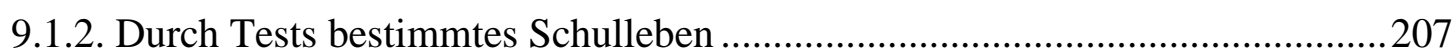

9.2. Kritik am „blinden Streben nach höherer Bildung“.........................................207

9.3. Das Scheitern der Bildungsreform von 1996 ..................................................209

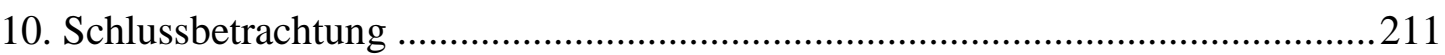

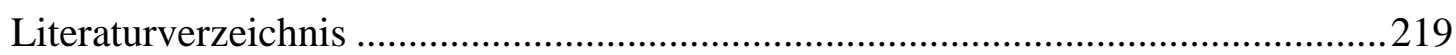


Verzeichnis der Abbildungen

Abbildung 1. Bildungssystem Ende der japanischen Besatzung

Abbildung 2. Bildungssystem 1949 in Taiwan

Abbildung 3. Bildungssystem gemäß den Pläne zur Ausbildung der Arbeitkräfte

Verzeichnis der Tabellen

Tabelle 1 Entwicklung der Anzahl der Schulen, Schüler und der Schulbesuchsrate in den Konfuzianischen Schulen und der Anzahl der Schulen sowie der Einschulungsrate in den „Öffentlichen Schulen“ von 1899 bis 1944

Tabelle 2 Wandel der Anzahl der taiwanesische Schüler in allen Bildungseinrichtungen von 1900 bis 1943

Tabelle 3 Anzahl der Schulen und der Schüler (Anzahl der gesamten Schüler, der taiwanesischen Schüler und der japanischen Schüler) und Anteil der taiwanesischen Schüler in den allen Bildungseinrichtungen im Jahr 1944

Tabelle 4 Entwicklung der Anzahl von Schulen, Klassen, Lehrern und Schülern sowie der relativen Schulbesuchsrate in der Grundschule von 1944 bis 1949

Tabelle 5 Entwicklung der Schülerzahlen in Junior High Schools, Junior Vocational High Schools, dreijährigen Lehrerausbildungsstätten, Senior High Schools und Senior Vocational High Schools, fünfjährigen pädagogischen Fachschulen, Fachschulen und Universitäten von 1945 bis 1949

Tabelle 6 Entwicklung der relativen Schulbesuchsrate, der Anzahl der Schulen, Schulklassen und Schüler sowie der durch schnittlichen Anzahl der Klassen und Klassenstärke in der Elementarbildung von 1950-1990

Tabelle 7 Entwicklung der Einschulungsrate in der Sekundarstufe I bzw. Junior High School und Junior vocational High School, Anzahl der Schulen, Schulklassen und Schüler sowie die durchschnittliche Anzahl der Klassen pro Schule und Klassenstärke in den Junior High Schools und Anzahl der Schulen und Schüler in den Junior Vocational High Schools von 1950 bis 1967

Tabelle 7a Die Schulbesuchsrate zu Grundschulen, Junior High Schools, Senior High Schools und Senior Vocational High Schools von 1950 bis 1990

Tabelle 8 Wandel der Anzahl der Schulen und Schüler an den Senior High Schools, Senior Vocational High Schools und Fachschulen von 1950 bis 1967

Tabelle 9 Entwicklung der Schülerzahlen in unterschiedlichen Lehrgängen in den Junior und Senior Vocational High Schools von 1952 bis 1967

Tabelle 9a Entwicklung der Schüleranzahl und Anteil der Schüler in unterschiedlichen Lehrgängen in den Junior und Senior Vocational High Schools in den Jahren 1952,1960 und 1967

Tabelle 10 Entwicklung der Anzahl der Fachschulen, Fachhochschulen und Universitäten sowie die Studentenzahl in den Hochschulen von 1950 bis 1990

Tabelle 11 Entwicklung der Einschulungsrate zur Sekundarstufe II, der Anzahl der Schulen, Schulklasse und Schüler sowie der durchschnittlichen Anzahl der Klassen pro Schule und der Klassenstärke in den Junior High Schools von 1968-1990

Tabelle 12 Entwicklung der Anzahl der Schulen und Schüler an den Senior High Schools, Senior Vocational High Schools und Fachschulen von 1968 bis 1990 
Tabelle 13 Entwicklung der Schülerzahlen in unterschiedlichen Lehrgängen in den Senior Vocational High Schools von 1968 bis 1990

Tabelle 13 a Entwicklung der Schüleranzahl und Anteil der Schüler in unterschiedlichen Lehrgängen in der Senior Vocational High School in den Jahren 1968, 1978 und 1986

Tabelle 14 Entwicklung der Anzahl der Schulen und Schüler in den gesamten und privaten Bildungseinrichtungen für Senior High Schools und Senior Vocational High Schools von 1968 bis 1990

Tabelle 15 Entwicklung der Anzahl der gesamten und privaten Bildungseinrichtungen und Studenten in den Hochschulen von 1950 bis 1990

Tabelle 16 Wandel der Anazahl der Prüfungsanmeldungen und der aufgenommenen Studenten, Aufnahmeraten zur Hochschulen von 1954 bis 2001

Tabelle 16 a Entwicklung der Prüfungsanmeldungen, der aufgenommenen Studenten und die Aufnahmerate zu den Hochschulen von 1967 bis 1983

Tabelle 17 Wandel der Prüfungsfächer in der Aufnahmeprüfung zur Hochschule von 1954 bis 1983

Tabelle 18 Anzahl und Anteil der angemeldeten und aufgenommenen Bewerber zur Universität im Jahr 1981 


\section{Einleitung}

Die vorliegende Arbeit will einen Beitrag zur historischen Forschung im Zusammenhang von Modernisierung und Erweiterung des staatlichen Bildungswesens in Taiwan im Zeitraum von 1885 bis 1987 leisten, d.h. von der Errichtung der Provinz Taiwan noch im chinesischen Kaiserreich bis zur Aufhebung des Kriegsrechts unter dem Regime der Nationalpartei Chinas (Kuomintang, abk. KMT). ${ }^{1}$

\subsection{Fragestellung und Forschungsstand}

Die Mitte des 18. Jahrhunderts in Westeuropa und den USA mit Modernisierung und industrieller Revolution einhergehende stark beschleunigte Entwicklung von Technologie, Produktivität und Wissenschaften führte zum Umbruch der politischen, wirtschaftlichen und sozialen Verhältnisse in der traditionellen Gesellschaft. Aufgrund wachsender funktionaler Differenzierung und fortschreitender Arbeitsteilung sowie der entsprechenden administrativen und organisatorischen Erfordernisse in der zunehmend urbanisierten Welt wuchs auch der Bedarf an kognitiven und kommunikativen Fähigkeiten wie Lesen, Schreiben und Rechnen.

Doch fand sich die Forderung nach einer allgemeinen Bildung für Jungen wie für Mädchen bereits grundlegend in Martin Luthers Schrift „An die Bürgermeister und Ratsherren aller Städte in deutschen Landen, daß sie christliche Schulen aufrichten

\footnotetext{
${ }^{1}$ Kuomintang, abgekürzt KMT oder GMD, Nationalpartei Chinas. Sun Yat-sen gründete im Jahr 1894, am Ende der Ching-Dynastie, in Honolulu, Hawaii, die „Hsing-chung Hui“, zu Deutsch „Gesellschaft für die Erneuerung Chinas"). Deren Ziel war es, die Mandschu-Regierung bzw. das Ching-Kaiserreich zu stürzen, um China zu reformieren. 1905 fusionierte die Hsing-chung Hui mit mehreren Revolutionsgruppen in Tokio. Nach der Revolution 1911 schloss Sun seine Partei mehrfach mit verschiedenen anderen politischen Parteien zusammen und gründete 1912 in Peking die Kuomintang. (Nationalpartei). 1914 organisierte sich in Tokio die KMT neu als Chung-hua Ke-ming Tang (Chinesische Revolutionäre Partei). 1919 wurde die Gruppe umbenannt in „Chung-kuo Kuomintang“, Nationalpartei Chinas. Vgl. Jürgen Domes 1970 und Chang Yuh-fa 1998.
} 
und halten sollen“ (1524). Nach Luthers Lehre sollte jeder Mensch Gottes Wort selbst lesen können, demzufolge sei Leseunterricht für jeden Christen vom Staat sowie Fürsten und Städten gemeinsam zu fördern und eine allgemeine Unterrichtspflicht einzuführen. ${ }^{2}$ In vielen protestantischen Fürstentümern wurde daraufhin „Leseunterricht für alle“ angeboten, z.B. 1642 in Gotha. Mit dem Generaledikt Friedrich Wilhelms I. von Preußen wurde im Jahr 1717 die Unterrichtspflicht eingeführt. Damit war Preußen einer der ersten europäischen Staaten mit Anspruch auf eine allgemeine Volkserziehung. ${ }^{3}$ Im Laufe des 18. Jahrhunderts griffen deutsche Aufklärungspädagogen das Konzept auf: Jeder Staatsbürger sollte die Bildung erhalten, die ihn in seinem Stande dazu befähigt, optimale Leistung für das Wirtschafts- und Wohlfahrtssystem des Staates zu erbringen, dies sollten eine Industrie- und Bauernschule für das einfache Volk, eine Real- und Fachschule für die bürgerliche Schicht sowie Ritterakademien als „Berufsschulen für den Adel“ anstelle der traditionellen Lateinschule leisten. ${ }^{4}$ Infolge von amerikanischer Unabhängigkeitserklärung (1776) und Französischer Revolution (1789-1794) wurde anschließend eine allgemeine Elementarbildung für die gesamte Bevölkerung als Aufgabe des Staates eingefordert und damit insgesamt ein Umwandlungsprozess in Gang gesetzt, von auf feudaler Gesellschaft und Religion aufbauender Erziehung und Bildung in ein vom Staat kontrolliertes Schulsystem als wesentliches Instrument zur Beseitigung des Analphabetismus, zur Erziehung zu mündigen und treuen Staatsbürgern sowie zur Ausbildung von Arbeitskräften. In Laufe des 19. Jahrhunderts strebten Länder wie Frankreich, Großbritannien, Preußen, die USA und Japan danach, ein öffentliches und staatlich kontrolliertes Bildungswesen aufzubauen und die Schulpflicht zu verordnen,

\footnotetext{
${ }^{2}$ Vgl. Friedrich Heman 1909, S. 43-47.

3 Diese war keine Schulpflicht, weil kein Schulbesuch gefordert wurde. Die Schulpflicht wurde in Deutschland erst mit der Weimarer Reichsverfassung von 1919 erlassen. Vgl. Herwig Blankertz 1982, S. 59 und Ulrich Herrmann 1993, (a) und (b), S. 563-582.

${ }^{4}$ Vgl. Herwig Blankertz 1982, S. 56-82 und Heinz-Elmar Tenoth 2000.
} 
so wie es heute weltweit Standard ist. ${ }^{5}$ Das bestehende Bildungswesen in Taiwan ist auch nach dieser Standard aufgebaut. Die Fragen dieser Arbeit sind folgend : wie das taiwanesische Bildungswesen im Laufe dieses Modernisierungsprozess von eigener bzw. konfuzianischer Erziehung zu einem modernen staatlichen Bildungswesen umgewandelt wurde?

Eine Auseinandersetzung mit der Beziehung zwischen konfuzianischer Erziehung in Taiwan und der modernen staatlichen Bildung westlicher Länder findet sich bei Forschern im englischen und deutschen Sprachraum. So beschäftigte sich etwa E. Patricia Tsurumi (1977) behandelte den Einführungsprozess der modernen Erziehung, vor allem der elementaren Bildung, während der japanischen Besatzung in Taiwan, ${ }^{6}$ Douglas C. Smith (1991) die Entwicklung der unterschiedlichen Bildungsphasen ${ }^{7}$ und Udo Dörnhaus (1988) den Aufbauprozess der Berufsausbildung nach Übersiedlung des KMT-Regimes, unterstützt durch die US-Entwicklungshilfe. ${ }^{8}$ Hsiao Chih-bang (2006) untersuchte den Wandlungsprozess in der Lehrerbildung von der konfuzianischen Tradition zur modernen professionellen Ausbildung ${ }^{9}$ und Liou Weichih (2006) den Prozess der Wissenstransformation von deutschen Bildungskonzepten ins chinesische bzw. taiwanesische Bildungssystem durch Pädagogikstudenten aus China in Deutschland. ${ }^{10}$

\footnotetext{
5 Nach den Untersuchungsergebnissen von John Meyer hatten im Jahr 1985 85\% aller Länder der Welt die Schulpflicht eingeführt. Vgl. John W. Meyer, Francisco O. Ramirez und Conrad W. Snyder 1992, S. 128-149.

6 Tsurumi, E. Patricia 1977: Japanese Colonial Education in Taiwan (1895-1945).

7 Smith, Douglas C. (Hrsg.) 1991: The Confucian continuum: educational modernization in Taiwan.

8 Dörnhaus, Udo 1988: Berufsbildungspolitik Taiwans im Verlauf der wirtschaftlichen Entwicklung (1949-1985).

${ }^{9}$ Hsiao Chih-bang 2006: Lehrerbildung als Politikum - ihre Befreiungs- und Professionalitätsprozesse in Taiwan - eine historische Darstellung.

${ }^{10}$ Liou Wei-chih 2006: „Aus deutschem Geistesleben ...“ Zur Rezeption der deutschen Pädagogik in China und Taiwan zwischen 1900 und 1960.
} 
Die vorliegende Arbeit fokussiert die Neugestaltung der Schulstruktur und die Prinzipien bei Erweiterung und Zuteilung von Bildungsplätzen in Taiwan, um daran den Umwandlungsprozess von konfuzianischer Erziehung zu moderner staatlicher Bildung zwischen 1885 und 1987 aufzuzeigen. Dazu wird folgenden Fragen nachgegangen:

1.Was ist konfuzianische Erziehung?

2.Wie sahen das kaiserliche Beamtenprüfungssystem und die konfuzianische Schule im traditionellen China aus?

3.Welche Wert- und Deutungsmuster wurden dadurch besonders in den Vordergrund gestellt?

4.In welchem historischen Zusammenhang wurde die Umwandlung von der konfuzianischen zur modernen staatlichen Bildung in Gang gesetzt?

5.Welche Organisationen und Individuen haben dazu beigetragen?

6.Welche Konzepte eines modernen staatlichen Bildungswesens wurden im Laufe der Zeit - unter Bezugnahme zahlreicher Strömungen und kontrovers diskutierter Bildungsansätzen - formuliert? Wie wurden diese schließlich umgesetzt?

7.Welche Probleme, welcher Widerstand und welches Versagen begleitete die Durchführung?

\subsection{Forschungsmaterialien}

Folgende Materialien wurden als Grundlage der hier vorgenommenen historischen und kritischen hermeneutischen Quellenanalyse genommen: Dokumente wie amtliche Edikte, die Jahrbücher der „Bildung Republic of China (ROC)“, ${ }^{11}$ statistische Angaben sowie Reden von Präsidenten und Bildungs- ministern, die wegweisende

11 ROC $=$ Republic of China 
Funktionen in der Bildungspolitik in Taiwan ausübten. Hinzu kommen Berichte einiger von internationalen Erziehungsberatern durchgeführter Forschungsprojekte, z.B. „The Reorganisation of Education in China 1932“ der Bildungskommission des Völkerbunds, „Education and Development - The Role of Educational Planning in the Economic Development of the Republic of China“" (1962) der Stanford Universität, „Bildungsplan der ROC - Langfristiger Bildungsplan“ der UNESCO von 1964 sowie „Pläne zur Ausbildung von Arbeitskräften“ der „Arbeitsgruppe Arbeitskräfteplanung“ des Wirtschaftsministeriums in den 1970er- und 1980er- Jahren, darüber hinaus sozialwissenschaftliche Untersuchungen, Magisterarbeiten und Dissertationen, die sich auf die Themenfelder Erweiterung und Kontrolle von Bildungsangeboten und Zuteilung von Bildungsplätzen beziehen. Auf relevante ausgewählte Fachliteratur, Bücher, Zeitschriften- bzw. Zeitungsartikel wird bei der Interpretation amtlicher Dokumente Bezug genommen. Eine weitere Informationsquelle stellen Angaben bzw. Daten aus dem Internet dar, welche die Beschreibung bzw. Analyse der Sachverhalte unterstützen sollen.

\subsection{Aufbau der Arbeit}

Die vorliegende Arbeit ist wie folgt aufgebaut: Zunächst werden die Grundgedanken des Konfuzianismus zur Erziehung, das kaiserliche Beamtenprüfungssystem und die konfuzianische Schule in China behandelt, um die Besonderheiten der traditionellen chinesischen Erziehung und Bildung auszuleuchten. Anschließend wird die Erziehung und Bildung von 1662 bis 1894 geschildert und dann der Umwandlungsprozess von konfuzianischer Erziehung zur modernen staatlichen Bildung unter dem chinesischen Ching-Kaiserreich und unter japanischer Kolonialherrschaft untersucht. Es folgt eine Untersuchung zur Gestaltung des modernen staatlichen Bildungswesens unter dem 
KMT-Regime von 1945 bis 1987: Erweiterung, Differenzierung und Verteilung der Schul- und Studienplätze. Abschließend werden der Kampf um Bildungsplätze und das Phänomen des von Chiang Kai-shek und den Kuomintang kritisierten „Shenghsueh chu-i“(升學主義, zu Deutsch: „blinden Bildungseifer“) ${ }^{12}$ vorgestellt und analysiert.

Die phonetische Umschrift der chinesischen Zeichen bzw. Sprache in die Phonetik des römischen bzw. lateinischen Alphabets richtet sich in dieser Arbeit nach dem Wade-Giles System, jedoch ohne vier Töne und Umlaute, das in Taiwan üblicherweise verwendet wird. Wenn die Autoren ihre Namen mit Hilfe eines anderen Systems umgeschrieben hatten, wird dies direkt übernommen.

12 „Sheng-hsueh“ bedeutet „,die eine weiterführende höhere Bildungsinstanz zu besuchen“. Der Ausdruck „Sheng-hsueh chu-i“ lässt sich schwer direkt ins Deutsche übertragen. Er ist sehr vieldeutig, weil die Inhalte davon abhängig sind, wie man die Probleme der schulischen Bildung versteht und interpretiert, außerdem wird er nach dem Wandel des schulischen Zustandes auf Taiwan immer wieder neu definiert. Im Allgemeinen beinhaltet dieser Begriffer eine geistige Haltung, wonach nicht die Bildungsinhalte das eigentlich Wichtige an der Erziehung darstellen, sondern das schulische Vorankommen, das Aufsteigen in die höhere Klasse bzw. in eine „bestmögliche“ höhere Schule und letztlich der Erwerb eines Diploms. 


\section{Konfuzianische Erziehung und das kaiserliche Beamtenprüfungssystem}

Die konfuzianische Lehre war im traditionellen China Grundlage von Staatslehre und Erziehung, so auch beim kaiserlichen Beamtenprüfungssystem. In diesem Kapitel sollen Grundgedanken des Konfuzianismus zur Erziehung und das kaiserliche Beamtenprüfungssystem vorgestellt werden.

\subsection{Grundgedanken des Konfuzianismus zur Erziehung}

Konfuzius (551-479 v. Chr.) wird als größter Philosoph und Pädagoge Chinas verehrt. ${ }^{13}$ Seine Lehre lässt sich als moralische wie auch politische Theorie auffassen, denn sie basiert auf Moralität. Die Legitimation der Herrschaft sollte sich nicht, wie in der feudalen Gesellschaft in seiner Zeit, auf verwandtschaftliche Abstammung gründen, sondern auf Persönlichkeit, Tüchtigkeit und Intelligenz. Das Staatsbeamtentum sollte sich für Jugendliche aus allen sozialen Schichten öffnen. Ein „Idealmensch“, ein „Edler“ oder „Gebildeter“ akzeptiere die harmonische Ordnung von Natur, Herrschaft und Familie, verehre Natur und Ahnen und sei loyal gegenüber dem Herrscher sowie ehrfürchtig vor den Eltern. Im Kapitel „Großes Lernen“ im „Buch der Riten“ wird darauf verwiesen, dass Bildung mit dem Erlernen von Grundkenntnissen in der Sittlichkeit wie den fünf Kardinaltugenden Humanität, Gerechtigkeit, geziemendes Benehmen, Weisheit und Vertrauen sowie der Kultivierung der Persönlichkeit beginne. ${ }^{14}$ Die Basis des sittlichen Lebens sei die familiäre Bindung, besonders wichtig sei kindliche Ehrfurcht und Liebe den Eltern sowie den Geschwistern gegenüber. Konfuzius suchte damit eine moralisch verantwortliche Lebensführung anzuregen: Die Liebe zu Eltern und Geschwistern erweitere sich später auch auf den Ehepartner, Verwandte, Freunde, Bekannte sowie auch

\footnotetext{
13 Vgl. Gregor Paul 2001 und Heiner Roetz 1995 und Yuen Chin-shu 1991.

14 Vgl. Chu Hsi 2007, S. 6-26.
} 
Unbekannte wie die Mitmenschen im Dorf, in der Provinz, im Reich und schließlich des gesamten Kosmos. Deswegen beruhten die Harmonie des Kosmos und die Stabilität bzw. Ordnung und Frieden des Staates auf der Moral der Einzelnen und der Harmonie in der Familie. Daher sei das Ziel der Erziehung wesentlich, dass die Schüler Verantwortung übernehmen und die erworbenen Fähigkeiten in den Dienst der Gemeinschaft stellen, allerdings erst dann, wenn sie wirklich in der Lage seien, mit ihren reichen Kulturkenntnissen und ihrer vervollkommneten Persönlichkeit als Vorbilder der Gemeinde, etwa als Beamte oder sogar auch als Herrscher des Kaiserreichs, zu wirken. ${ }^{15}$

Nach Konfuzius sind alle Menschen bildungsfähig und formbar, er forderte daher den Zugang zur Bildung für alle. Der Gedanke der Klasse in der Erziehung sollte eliminiert werden, Lehren und Lernen geschehe ohne Ansehen der Klassenunterschiede, Erziehungschancen gebührten allen, die nach Bildung streben, ohne Rücksicht auf Herkunft und soziale Stellung. ${ }^{16}$ Erziehung zielt nach Konfuzius neben der Schulerziehung auf alltägliche Bereiche wie den familiären Umgang, gesellschaftliche Kontakte und persönliche Gespräche. Jedes Individuum sollte nach seinen Talenten und Fähigkeiten von Nutzen sein, die Schüler sollten ihren Begabungen gemäß erzogen und ihnen entsprechende Lehrinhalte vermittelt werden. ${ }^{17}$ Bücher verschiedenster Art sollten gelesen werden, um sich mit diversen Interessengebieten zu befassen. Als grundlegende Lehrinhalte galten dabei neben den sechs Grundfähigkeiten Sittlichkeit, Musik (Rhetorik), Bogenschießen, Reitkunst und Wagenlenken, die Kalligraphie (Lesen und Schreiben) sowie Mathematik (Arithmetik und Astrologie). Hinzu kamen sechs Standardklassiker: „das Buch der Lieder“, „das Buch

\footnotetext{
15 Vgl. Kapitel von Tzu-chang im Lun-yu.

16 Vgl. Kapitel von Wei-ling-gong im Lun-yu.

17 Vgl. Kapitel von Wei-cheng im Lun-yu.
} 
der Urkunde“, „das Buch der Riten“, „das Buch der Wandlungen“, „das Buch der Musik“ und „die Frühlings- und Herbstannalen“. ${ }^{18}$

\subsection{Das kaiserliche Beamtenprüfungssystem}

Konuzianismus zufolge sollten Moralität und Kulturkenntnisse einer Person als Kriterien für die Berufung eines Beamten im Vordergrund stehen, sondern nicht Abstammung und Familiehintergrund. Jedoch wie wurde dies politische Ideal in der Praxis im alten China umsetzt? Das kaiserliche Beamtenprüfung galt als eine Verkörperung dieses politischen Ideals im alten China. In diesem Abschnitt sollen die Entstehung und das System der kaiserlichen Beamtenprüfung geschildert werden.

\subsubsection{Die Entstehung des kaiserlichen Beamtenprüfungssystems}

Der Konfuzianismus wurde in der Han-Dynastie (202 v. Chr. bis 220 n. Chr.) zur Staatsdoktrin erhoben und damit auch zum Leitprinzip bei der Ausbildung und Auswahl von Beamten. ${ }^{19}$ Inzwischen sollte die Auswahl der Anwärter außerhalb des Vererbungssystems auf Empfehlung sowie durch Abfragen konfuzianischer Kenntnisse getroffen werden. ${ }^{20}$ Im Jahr 606 wurde auf Befehl des Kaisers Yang (560-618) in der Sui-Dynastie (581-618) eine zentrale kaiserliche Beamtenprüfung offiziell gestaltet ${ }^{21}$ und während der Tang-Dynastie (618-907) erweitert. Neben

\footnotetext{
${ }^{18}$ Vgl. Hu Shih-ching 2009, S.548-558.

${ }^{19}$ Der konfuzianische Gelehrte der Han-Dynastie, Tung Chung-shu (179-104 v. Chr.), hatte dem damaligen Kaiser Wu (156-87 v. Chr.) vorgeschlagen, die anderen seit der „Frühlings- und Herbstperiode“ (770-476 v. Chr.) sowie der Zeit der Streitenden Reiche (475-221 v. Chr.) entstandenen konkurrierenden Lehren auszuschalten und dem Konfuzianismus den Status als herrschende Staatslehre zu verleihen. Vgl. Li Hsin-ta 1995, S. 26f.; Wang Bing-chao und Hsu Yung 2002, S. 118-125.

20134 v. Chr. wurde das Empfehlungssystem gestaltet. Die hohen oder lokalen Beamten, die für die Auswahl der neuen Beamten zuständig waren, suchten die kompetenten Beamtenkandidaten in Reichweite mit Rücksicht auf ihre Moralität und literarischen Tätigkeiten und nach dem proportionalen Anteil der Bevölkerung des Landes aus. Dies ermöglichte einen Aufstiegsweg für kompetente Literaten in den Dienst des Kaiserreiches und zur Übernahme der wichtigen Aufgaben des Kaiserreiches, diese durften aber nicht aus einer Beamtenfamilie stammen. Vgl. Benjamin A Elman 2000, S. 5f..

${ }^{21}$ Vgl. Li Hsin-ta 1995, S. 105-110.
} 
Vererbung, Empfehlung, persönlicher Beziehung und Ämterkauf erlaubte es nun duch eine Reihe von öffentlichen komplizierten Prüfungsverfahren, in den Staatsdienst eintreten zu dürfen. ${ }^{22}$ Damit wurde Konfuzius' politisches Ideal, dass die politische Macht durch Leistung und Tüchtigkeit und nicht durch Vererbung oder Abstammung bestimmt werden solle, radikaler als zuvor praktiziert. Das System wurde erst kurz vor dem Verfall des Ching-Kaiserreichs (1644-1911), im Jahre 1905, aufgehoben. ${ }^{23}$

\subsubsection{Die Stufung des kaiserlichen Beamtenprüfungssystems}

Seit dem 6. Jahrhundert wurde das kaiserliche Beamtenprüfungssystem allmälich zu wesenlicher Methode der Auswahl der Beamten, zugleich wurde ein anspruchvolles Prüfungssystem in der chinesischen Geschichte entwickelt. Im Allgemein folgten die kaiserlichen Beamtenprüfungen einem festen Verlauf: Nach der elementaren Erziehung in der Familie bzw. in der lokalen, privaten Schule nahm ein Kandidat zunächst an einer lokalen Prüfung, manchmal auch „Prüfung für junge Schüler“ genannt, teil, d.h. einem Bezirks-, Präfektur- und Qualifikationsexamen innerhalb des Landkreises. Diese berechtigte ihn noch nicht zum Eintritt in den Beamtendienst, sondern lediglich zum Besuch der lokalen amtlichen Schule, wo er neue Instruktionen erhielt und sich auf die künftigen Prüfungen vorbereiten konnte. Mit dem Bestehen der ersten Stufe gewann der Kandidat den Lizenziatenstatus und damit den Amtstitel Hsiu-tsai (zu Deutsch ,ausgezeichnetes Talent“ oder „,ausgesuchter Schüler"),. Anschließend durfte er an den folgenden Prüfungen, den Provinz-, Hauptstadt- und Palastexamen teilnehmen. Deren erfolgreiche Kandidaten trugen die Titel Chu-jen, (zu Deutsch „empfohlener Beamtenkandidat“); die höhere Titel wie Kung-shih (zu Deutsch ,ausgesuchter Literat“), und Chin-shih (zu Deutsch

\footnotetext{
22 Vgl. Denis Crispin Twitchett 1976; Li Hsin-ta 1995, S. 99-131 und Kao Ming-shih 2006,S.181-215.

23 Vgl. Chang Ya-chun 2005.
} 
„hervorragender Literat"“) und konnten nun als Beamter berufen werden. ${ }^{24}$

\subsubsection{Die Prüfungsinhalte}

Die Prüfungsfächer waren in allgemeine und Spezialfächer unterteilt. Zentraler Bestandteil der allgemeinen Prüfungen waren die Werke des Konfuzianismus wie „Vier Bücher“ des Konfuzianismus: „Lun-yu“, die Analekte des Konfuzius, gesammelte Gespräche mit seinen Schülern, „Meng-tzu“, Sammlung der Gespräche von bedeutenden Nachfolgern von Konfuzius, „Ta-hsueh“, das Buch des großen Lernens, eine Erziehungsfibel für die gehobenen Stände und die höhere Bildung, und „Chung-yung“, das Buch von Mitte und Maß, sowie die „Fünf Klassiker“ „das Buch der Lieder“, „das Buch der Urkunde“, „das Buch der Riten“, „das Buch der Wandlungen“ und „die Frühlings- und Herbstannalen“, welche seit der Han-Dynastie zum verbindlichen Kanon für Gelehrten erhoben wurden. ${ }^{25}$ In der Nordlichen Song-Dynastie 960-1127) kamen 1061 noch einige Prüfungsinhalte hinzu, insgesamt wurden die Werke nun als „dreizehn Klassiker“ bezeichnet: Buch der Wandlungen, Urkunden, Lieder und Riten, Letzteres unterteilte sich in die Beamten und Riten der Chou (die konfuzianischen Riten des Königreichs Chou), ${ }^{26}$ Zeremonien und Aufzeichnungen über Riten, sowie die Annalen von Tso Chuan, Kung-yang Chuan, Ku-liang Chuan, Lun-yu, Hsiao-ching, Meng-tzu und Erh Ya. ${ }^{27}$ Währen der Yuan-Dynassite (1271-1368), im Jahre 1314 kamen die Kommentare der „Vier Bücher“ und „Fünf Klassiker“ von wichtigen Vertretern des Neo-Konfuzianismus ${ }^{28}$

\footnotetext{
${ }^{24}$ Vgl. Ichisada Miyazaki 1981, S. 18-101.

25 Vgl. Wolfgang Bauer 2006, S. 46.

${ }^{26}$ Dieses wahrscheinlich erst zur Zeit um Jahre 9-23 verfasste Buch beschreibt sehr sorgfältig und detailliert die konfuzianischen Riten des Königreichs Chou, wobei im Mittelpunkt Riten der Staatsführung stehen. Vgl. Hu Shih-ching 2009 S. 550.

27 Zur Wandlung der standardisierenden Lerninhalte siehe Lee Thomas H.C. 2000, S. 363-430.

${ }^{28}$ Neo-Konfuzianismus ist eine Denkrichtung des Konfuzianismus, er wird der Song-Dynastie aktiv praktiziert. Die Neo-Konfuzianisten beschäftigten sich besonders mit den Themen bzw.die Heraus-
} 
in Südliche Song-Dynastie, Chu Hsi (1130-1200) ${ }^{29}$ hinzu. Später, im Laufe der Ming- und Ching-Dynasite wurde die Werke und Kommentare zu konfuzianischen Klassiker von Chu Hsi zur Standarauslegung in der kaiserlichen Beamtenprüfung angenommen.

Mitte der Ming-Dynastie (um 1600) kamen zahlreicher jesuitischer Missionare wie Metteo Ricci (1581-1610), Johann Adam Schall von Bell (1591-1666), P. Ferdinand Verbist (1623-1688) u. a. in China an. Die jesuitischen Missionare propagierten nicht nur das Christentum, sondern auch die mittelalterlichen Naturwissenschaften wie Astronomie, Geometrie, Medizin, Physik, Philosophie usw. am chinesischen Kaiserhof. ${ }^{30}$ Unter dem Einfluss der Jesuiten wurde seitdem eine sogenannte „pragmatische Lehre“, die sich auf Technik und naturwissenschaftliche Forschung konzentrierte, ${ }^{31}$ unter den chinesischen Gelehrten entwickelt wurden die Themen der Astrologie, Mathematik sowie Naturwissenschaft in der Zeit auch in den kaiserlichen Prüfungen vorgekommen. ${ }^{32}$

\subsubsection{Die Prüfungsformen}

Obwohl jede Dynastie unterschiedliche Schwerpunkte bei den Prüfungen setzte, wurden die Prüfungsformen zumeist in drei Bereiche gegliedert: allgemeine

forderung aus dem Budhisten zu antowrten: die Rolle der Menschen im Kosmos und das Gesetz der Natur und Menschheit. Sie versuchten ihre Lösung außer dem Konfuzianismus in den Lehren von Taoismus und Buddhismus zu finden. Vgl. Hu Hsi-ching 2009, S.575-585.

${ }^{29}$ Chu Hsi (1130-1200) war der wichtigste Vertreter des Neo-Konfuzianismus, und lehrte an der berühmten Akademie der Weißen Hirsch-Grotte. Er kommentierte die Werke des Konfuzianisten und stellte die neuen Konzepte für die elementare und höhere Erziehung dar. Er war Lehrer und Berater des Song-Kaisers. Vgl. Chen Jung-chieh 1990.

30 Vgl. Wang Chung-fu1981, S.501-507; Hsu Kuang-tai 2003, S. 445-471 und ders. 2004, S. 77-104.

31 Vgl. Shen Ting-ping 1994, S. 87-117; ders. 2001.

32 Vgl. Benjamin A. Elman 2005. 
Kenntnisse der klassischen Lektüren, Debattiertaktik und Dichtkunst. Für jede Ebene gab es drei Sitzungen: In der ersten Sitzung wurde das Verständnis der konfuzianischen Klassiker geprüft, in der zweiten der Diskurs, das Schreiben von amtlichen Ankündigungen und die richterlichen Entscheidungen. In der dritten Sitzung standen die Auseinandersetzung mit einem politischen Thema und der Kommentar zu einem bestimmten historischen Ereignis oder zu einer bestimmten juristischen Fall oder die Erstellung einer Lösung für akute soziale Frage oder übertragbare Krankheit im Mittelpunkt; manchmal wurden Gedichte $\mathrm{zu}$ vorgegebenen Themen und nach vorgegebenem Versmaß abgefasst. ${ }^{33}$

In der Ming-Dynastie (1368-1644) instrumentalisierte der Kaiserreichgründer Chu Yuen-chang (1328-1398) das kaiserliche Beamtenprüfungssystem für die Vereinheitlichung der öffentlichen Meinung. Die Prüfungsformen wurden daher formeller und einheitlicher als zuvor gestaltet. Ab 1371 wurde eine neue Prüfungsform, der sog. „Pa-ku-wen“ (zu Deutsch: „,achtgliedriger Aufsatz“), ${ }^{34}$ mit starker Ritualisierung und Reglementierung eingeführt. Darin wurden detaillierte Kenntnisse der Klassiker sowie bestimmte stilistische Fertigkeiten abgefragt. ${ }^{35}$

\footnotetext{
${ }^{33}$ Vgl. Benjamin A. Elman und Alexander Woodside 1994, S. 118-123, sowie Li Hsin-ta 1995, S. 277f.

${ }^{34}$ Der hohe Beamte und Gelehrte Wang An-shih (1021-1086) forderte schon in den 1070er-Jahren eine Standardisierung der kaiserlichen Beamtenprüfung. Der Zweck war, damit die Moralität der Prüfungsteilnehmer zu stärken. Diese sollten einerseits die Inhalte der konfuzianischen Lektüren gut beherrschen, andererseits in diesem Sinne die realen sozialen Probleme interpretieren oder Lösungsvorschläge finden. Mit der Standardisierung sollten Prüfungslektüren und Korrekturkriterien festgehalten werden. Wang hatte die Argumentation zum Vorbild vorgelegt, was später als Urform von „Pa-ku-wen“genommen wurde. In der Regel sollte „Pa-ku-wen“ nach Bestimmungen in der ChingDynastie mit einem Wortschatz von 700 Worten geschrieben werden; Themen waren die Lehren des Konfuzianismus. Jeder der acht Abschnitte hatte eine festgelegte Funktion (etwa Einleitung, Eröffnung der Diskussion, Abstraktion, Positve Argumentation, Vertiefende Argumentation, Gegenargumentation, Synthese, Schlussworte usw.), und es gab gesonderte Regeln für jeden Teil, etwa zur Anzahl der Sätze, zu Format und Stil, sowie Reimgesetze, Symmetrieregeln und Ähnliches. Kaiser Chien-long (17111799) hatte Sammelbände der Standardaufsätze zur Nachahmung für Prüfungsteilnehmer veröffentlicht. Vgl. Kong Ching-mao 2008, Liu Hong 2006, S. 82-93, Lee Thomas H.C. 2000, S. 159-161 und Benjamin A. Elman 2010, S. 194-227.
}

35 Vgl. Chien Mu 1977, S. 92f. 


\subsubsection{Voraussetzungen für das Teilnehmen an die kaiserliche Beamtenprüfungen}

Obgleich der Zugang zum Beamtenstatus allen sozialen Schichten offen stehen sollte, verfügten tatsächlich nur Männer, die aus einer gehobenen und angesehenen Familie stammten oder entsprechende finanzielle Unterstützung erhielten, über die geforderte umfangreiche Vorbildung. Behinderte, Kranke, Strafgefangene, Schauspieler, Sklaven, buddhistische und taoistische Mönche, Händler sowie auch Frauen und Mädchen waren von vornhinein ausgeschlossen. ${ }^{36}$ Auch wenn die Eltern kurz vor der Prüfung gestorben waren, durften sich diese Kandidaten nicht zur Prüfung melden. ${ }^{37}$

\subsubsection{Anzahl der Bewerber}

Die Prüfungen fanden alle drei Jahre statt, z. B. in der Ching-Dynastie wurden in der ersten Stufe ca. 30.000 Kandidaten, in der zweiten ca. 1.500 und in der dritten ca. 300 aufgenommen. ${ }^{38}$ Die Menge hing zunächst von der Bevölkerungszahl in den Herkunftsprovinzen ab. In der letzten Stufe der Prüfung wurde die Anzahl der aufgenommenen Bewerber nicht mehr festgesetzt, sondern hing von der Qualität der Bewerber ab. Dadurch konnten die Beamten relativ gleichmäßig aus den verschiedenen Provinzen ausgewählt werden. ${ }^{39}$ Nach der Berechnung von Chang Chung-li (1955) lag die Quote um 1860 bei der Provinzprüfung in der Ching-Dynastie bei ungefähr 1,5\% (ca. 2 Millionen Prüfungsteilnehmer konkurrierten um 30.000 Plätze), die Quote für aufgenommene Bewerber in der Hauptstadtprüfung lag bei ca. $20 \% .^{40}$

\footnotetext{
36 Vgl. Chou Yu-wen 2001, S. 132f..

37 Nach konfuzianischer Sitte sollten die Kinder mindestens drei Jahre nach dem Tode ihrer Eltern an den öffentlichen Aktivitäten nicht teilnehmen, dadurch ihre Kindespietät zu äußern.

38 Vgl. Wang Yi-chu 1966, S. 13.

39 Vgl. Edward A. Kracke 1957, S. 298 und Chou Yu-wen 2001, S. 146-151.

40 Vgl. Chang Chung-li 1955, S. 88-94.
} 


\subsection{Schuleinrichtungen und Bildungsinhalte}

Nach der Einführung des kaiserlichen Beamtenprüfungssystems wurde die Bestehen der kaiserlichen Beamtenprüungen nach und nach zur wichtigsten Voraussetzung für die Bewerbung um eine Beamtenstelle. Im Laufe der chinesichen Geschichte verbreitete sich die sog. „,konfuzianische Schule“, eine Schulform zur Vermittlung der konfuzianischen Lehre und entsprechender Lektüren sowie zur Vorbereitung auf die kaiserlichen Beamtenprüfungen. Im diesen Abschnitt sollen unterschiedliche Einrichtungen und Lehrinhalte der Erziehung und Bildung im traditionellen China vorgestellt werden.

\subsubsection{Einrichtungen und Lehrinhalte der elementaren Erziehung und Bildung}

Die konfuzianische Erziehung stand seit Han-Dynasite nur gehobenen sozialen Gruppen offen. ${ }^{41}$ Bis zum 6. und 7. Jahrhundert übten die ausgebildeten Beamten und die gehobenen Familien und Sippen die sogenannte „Familien- und Sippenerziehung“ aktiv aus. Durch die „Familien- und Sippenerziehung“ überlieferten die Gebildeten mit ihrer umfangreichen konfuzianischen Kenntnissen zu ihren Nachwuchsen der Familienn und Sippen, ${ }^{42}$ sowohl Jugend als auch Mädchen. Zugleich

41 Vgl. Kao Ming-shih 2005, S.1-420.

42 Der Umfang einer Familie oder Sippe in altem China konnte sich auf die ganze Gemeinde bzw. das ganze Dorf beziehen und aus zehntausenden Mitgliedern bestehen. Die Familie beschränkte sich mit einigen Dutzend Menschen unter einem Dach aber auf die Oberschicht, normalerweise bestand die Familie aus Elternpaar, Kindern und Großeltern. Die Angehörigen einer Sippe stammten väterlicherseits von demselben Ahnherrn ab, trugen denselben Familiennamen und lebten gewöhnlich in derselben Gegend, oft im gleichen Dorf zusammen. Die Größe der Sippe war unterschiedlich, teils umfasste sie ein paar hundert Mitglieder, teils mehrere tausend. Entscheidend war, ob ein Sippenangehöriger es zu Amt und Würden gebracht hatte, denn Einfluss und Reichtum kam allen Sippenmitgliedern zugüste. Alle hatten Anspruch auf Unterstützung durch die Sippe. Zu den ideellen gehörte vor allem die Ahnenverehrung, das gemeinsame Feiern von Festen, die Führung genealogischer Bücher der Sippen; zu den materiellen Aufgaben die Unterhaltung von Schulen, die Unterstützung alter oder kranker, die Ausbildung begabter Sippengenossen, die man auch auf Kosten der Sippe zu den privaten Prüfungen schickte, die Sorge für Bewässerungsanlagen, Dämme, Brücken und andere Bauarbeiten. Weiter wachte die Sippe über Sitte und Ordnung und bestrafte Missetäter aus ihren Reihen, in extremen Fällen sogar mit dem Tode. Sie bildete in Handwerk, Handel und Landwirtschaft im Normalfall eine Produktionseinheit. Für gute Söhne und ehrbare Witwen errichtete die Sippe Gedenktore; sie schlichtete Streitigkeiten unter ihren Mitgliedern und stellte häufig sogar einen 
förderten und forderten sie ihre Mitglieder der Familien und Sippen der Sitte und Normen des Konfuzianismus zu praktizieren. ${ }^{43}$

Mit der Einführung des kaiserlichen Beamtenprüfungssystems waren weite Teile der Bevölkerung motiviert, an den kaiserlichen Beamtenprüfungen teilzunehmen, ${ }^{44}$ da mit Bestehen der Prüfungen erhebliche politische Macht, Reichtum, Privilegien und soziales Ansehen verbunden war. Die Vorteile begünstigten nicht nur die Prüflinge selbst, sondern auch deren Familie, indem sie von bestimmten Steuern und körperlichen Strafen befreit waren; zudem wurde die Teilnahme an lokalen Angelegenheiten ermöglicht. ${ }^{45}$ Weiterhin ließ sich der politische Einfluss einer Familie durch den Erfolg des Nachwuchses verstärken. Daher wurde dieser nicht nur als Leistung einer einzelnen Person, sondern auch als große Ehre und Stolz der gesamten Familie angesehen. ${ }^{46}$ Außerdem bestand nur eine geringe Anzahl die komplizierten Beamtenprüfungen, genossen die Erfolgreichen deswegen ein erhebliches soziales Ansehen, selbst wenn sie nicht ins Amt berufen wurden. Der Bedarf an konfuzianischer Erziehung und Bildung bzw. konfuzianischen Schulen wurde daher immer mehr zugenommen.

Währen der Sui- und Tang-Dynastie (618-906) und Song-Dynastie (960-1279) gestaltete das Kaiserreich nicht nur das Beamtenprüufngssystem, sondern setztete

bewaffneten Selbstschutz auf, eine Art Sippenmiliz. Als materielle Basis für ihre zahlreichen Ausgaben diente der Landbesitz, welcher der Sippe als solcher gehörte (also unabhängig vom Privatbesitz der einzelnen Mitglieder) und zumeist aus Stiftungen reicher Mitglieder bestand. Vgl. John C.H. Fei und Liu Tsui-jung 1982, S. 375-408.

43 Vgl. Kao Min-shih 2005, S.444-466.

44 Anfang des 11. Jahrhunderts gab es jedes Mal 20.000 bis 30.000 Prüfungsteilnehmer an der lokalen Beamtenprüfung, bis Mitte des 13. Jahrhunderts waren es über 40.000. Vgl. John W. Chaffee 1995, S.33-35.

${ }^{45}$ Vgl. ebd. S. 3-27 und 194-230. Zur Familienerziehung und Reproduktion der konfuzianischen Lehre durch Familienerziehung siehe Lee Thomas H.C. 2000, S. 477-521.

${ }^{46}$ Vgl. Brian E. Mcknight 1971, S. 178-185; Yang Mao-chun 1988, S. 133-167; Wen Chung-i 1988, S. 49-90 und 133-167; Hsiung Ping-chen 1992, S. 201-238. 
auch massive Bildungsmitteln ein, um amtliche Schulen zu errichten. Zugleich mit der Verbreitung des Buddhismus boten die buddhistischen Tempel eine elementare Bildung für Mönche, Nonnen und Laien an, damit sie sowohl buddhistische als auch konfuzianische Texte lesen und studieren konnten. ${ }^{47}$ Es gab auch in dieser Zeit zahlreichen ausgebildeten Einsiedler, die Unterrichten gaben. Die Jugendlichen aus den bildungungüstigen Familien gewannen dadurch die Gelegenheit, konfuzianische Lektüren $\mathrm{zu}$ studieren und an die kaiserliche Beamtenprüfung teilnehmen $\mathrm{zu}$ könnnen. ${ }^{48}$ Zudem unterstützten die erhobenen Familien und Sippen oder Geschäftsleute gern die Errichutng der Bildungseinrichtung erheben, weil ihre soziale Position und Ansehen dadurch erhöhen konnten.

Es gab drei Schulformen für die elementare Bildung: Die erste und am meisten verbreitete Schulform war die private Schule (Ssu-shu) oder die Studienzimmer (Shu-fan), die entweder von den konfuzianischen Gelehrten selber den Unterrichten organisiert werden oder von den Familien oder Sippe für ihren Nachwuchs errichtet wurden. ${ }^{49}$ Die zweite Schulform war die amtliche Gemeindeschule (She-hsueh), die, von der Provinzregierung finanziert, die elementare Bildung förderte, doch wurde keine Schulpflicht praktiziert. Eine weitere Schulform war die Sponsoren- oder Wohlfahrtsschule (I-hsueh) für Kinder aus hilfsbedürftigen Familien. Sie wurden entweder von der Provinzregierung oder von wohltätigen Stiftungen durch reiche Gelehrte und Großgrundbesitzer wie auch von den buddhistischen Tempeln finanziert. ${ }^{50}$ In der Regel waren die konfuzianischen Schulen nicht groß, aber relativ zahlreich. Der Unterricht fand häufig entweder im Haus eines Lehrers, der Schüler

\footnotetext{
47 Vgl. Erik Zürcher 1989, S. 19-56 und Lee Thomas H.C. 1989, S. 105f.

48 Vgl. Kao Min-shih 2005, S. 466- 488.

49 Vgl. Hu Shih-ching 2009, S. 299.

${ }^{50}$ Vgl. Chou Yu-wen 2001, S. 346-354.
} 
oder aber in einem Raum bzw. einer Ecke im Tempel statt.

Die Lehrkräfte der konfuzianischen Schulen waren oft Gelehrte, die die kaiserliche Beamtenprüfung abgelegt hatten oder sich auf die nächste Stufe der kaiserlichen Beamtenprüfung vorbereiteten. Sie waren von jeglicher körperlicher Arbeit befreit und wurden vom Staat gefördert, zugleich standen sie unter kaiserlicher Aufsicht. ${ }^{51}$ Wie Ersatzväter verehren, standen sie häufig in enger Beziehung zu den Schülern und deren Familien und genossen ein hohes soziales Ansehen in ihren Gemeinden.

Mit der Ausweitung der elementaren Bildung besuchten immer mehr Kinder aus sozial niedrig stehenden Familien die Schule. Es bedurfte daher neuer Lehrstoffe und einer neuen Didaktik für sie. In der Südlichen Song-Dynastie (1127-1279) erstellte der bedeutende Philosoph Chu Hsi (1130-1200) ein Programm, das sog. „Kleine Lernen“ (Hsiao-hsueh), mit kindgemäßer, für die breite Masse der Bevölkerung passender und praxisbezogener Pädagogik. ${ }^{52}$ Die Phase vom „Kleinen Lernen“ ist im Alter von acht bis fünfzehn Jahren. Im „, Kleinen Lernen“ gab es imsgesamt 385 Themen. Es beinhaltete zunächst grundlegende Benimmregeln und die Verinnerlichung konfuzianischer Tugenden durch alltäglichen Umgang, um dann Gedichte und Klassiker auswendig zu lernen, wenngleich die Schüler den Inhalt nicht verstanden. Im Laufe der Zeit wurden zahlreiche Bücher für Kinder bzw. Lernanfänger geschrieben, z.B. „Drei-Zeichen-Klassiker“, „Tausend-Zeichen-Klassiker“, „Gedichtsammlung von Tausenden Dichtern“, „Hunderte Familiennamen“, „Regeln für Kinder“ sowie „Lektüre für die Kindererziehung“, die aus kleinen Texten in Form von Gedichten zur Vermittlung der Grundkenntnisse und Sittlichkeit sowie zur

\footnotetext{
51 Vgl. Hu Shih-ching 2009, S.312f.

52 Vgl. M. Theresa Keller 1989, S. 219-251; Wu Pei-yi 1989, S. 370-324 und Hsu Tzu 2006, S.57-282.
} 
Ausbildung der Fähigkeit des Dichtens zusammengestellt waren. ${ }^{53}$ Diese Kinderbücher waren die Grundlage für das Lernen der 5000 chinesischen Zeichen.

In der Praxis lag das Einschulungsalter bei acht Jahren, es gab jedoch keine altersmäßige Einteilung in Klassen, ebenso wenig einen festgelegten Lehrplan. Zudem war es für die Schüler möglich, nur sporadisch am Unterricht teilzunehmen. ${ }^{54}$ Die Lehrinhalte richteten sich häufig nach den Wünschen der Eltern oder der individuellen Entwicklung der Kinder. Sie dienten entweder zur Vorbereitung auf die kaiserliche Beamtenprüfung oder anderen pragmatischen Zwecken: Briefe lesen und schreiben, amtliche Edikte verstehen, Quittungen und Rechnungen erstellen sowie die Regeln der sozialen Ordnung einhalten. ${ }^{55}$ Nachahmung, Rezitation und ständige Wiederholung waren dabei die wesentlichen Lerntechniken. ${ }^{56}$

\subsubsection{Einrichtungen und Lehrinhalte der höheren Bildung}

Nach dem „Kleinen Lernen“ c.a. Beginn mit dem Alter von fünfzehn Jahren durften die Jugendlichen sich mit beim „Großen Lernen“ (Ta-hsueh) bzw. mit dem Studium der „Vier Bücher“ und „Fünf Klassiker“ sowie mit umfangreichen Lektüren über die chinesische Geschichte usw. beschäftigen und auch Aufsätze schreiben; hinzu kamen Kalligraphie, Auslegung der Klassiker und Annalen, Dichten, Rechnen usw.. ${ }^{57}$ Dadurch bildeten die Schüler nicht nur das rationale Denken und das ästhetische Geschmack aus, sondern verinnerlichten auch die moralischen und politischen

\footnotetext{
${ }^{53}$ Zur Entwicklung der Lehrstoffe für elementare Erziehung siehe Chou Yu-wen 2001, S. 361-370 und zur Entwicklung der Einstellung zur Didaktik der elementaren Erziehung siehe Chou Yu-wen 2001, S. 370-380.

${ }^{54}$ Vgl. Hsu Pei-hsien 2001, S. 207.

55 Vgl. ebd., S. 263f.

56 Vgl. Chou Yu-wen 2001, S. 355-359.

57 Vgl. John W. Chaffee 1995, S. 5f.; Peter K. Bol 1989, S. 151-185 und Wm. Theodore de Bary 1989, S. 186-218.
} 
Führungen des Konfuzianismus bzw. Kaiserreichs. Daneben etablierten sich Formen der Fachausbildung, etwa der Naturwissenschaften mit klassischer Astronomie und Astrologie oder der chinesischen Medizin und Geographie, außerdem fanden in amtlichen Fachschulen und Instituten für den Dienst am kaiserlichen Hof Lektionen in Kampfkunst, Malen, Rechnen und Musik statt. ${ }^{58}$ Jedoch beschränkte sich die sog. „Militärerziehung“ auf die Kampfkunst wie Bogenschießen und Reiten sowie auf das Studium von Strategie und Taktik der Kriegsführung in der höheren Bildung. Denn das Kaiserreich verbot eine private militärische Ausbildung, um schlagkräftige Aufstände zu vermeiden. Sportunterricht und Militärerziehung wurden daher selten angeboten. $^{59}$

Nach Bestehen der Prüfung bekamen die Schüler Plätze in den amtlichen konfuzianischen Schulen, die meist staatlich finanziert wurden, um die nächste Stufe der Prüfung vorzubereiten. Mit der erfolgreichen Absolvierung des Unterrichts und der Prüfungen durften die Schüler an der nächsten Stufe der Beamtenprüfung teilnehmen. Die vom Kaiserreich errichteten Einrichtungen für die höhere Bildung gab es folgend: Die „Han-lin Akademie“ (Han-lin Yuen), ${ }^{60}$ war die höchste Akademie des chinesischen Kaiserreichs, deren Mitglieder oft unter den Chin-shih (hervorragenden Literaten), die den Palastexamen abgelgt haben, ausgesucht wurden. ${ }^{61}$ Ferner gab es Kollegs (Kuo-tzu-chien) für die Kinder der Kaiser, der Adeligen und der Hohen Beamten, kaiserliche Schulen für höhere Studien (Tai-hsueh) sowie unterschiedliche, nach Landkreisen und Provinzen abgestufte Bildungs-

\footnotetext{
58 Vgl. Lee Thomas H.C. 2000, S. 521-541.

59 Vgl. Chen Fang-ping und Bi Chen 1996, S. 331.

${ }^{60}$ Diese war eine Einrichtung, die u. a. Schreib- und literarische Aufgaben für den Kaiserhof ausführte. Nur die erlesensten Gelehrten durften Mitglieder der Akademie werden. Eine ihrer Hauptaufgaben war, Entscheidungen über die Auslegung der konfuzianischen Klassik zu treffen. Diese bildeten die Basis für die Beamtenprüfungen, die wiederum den Zugang zu den höheren Beamtenrängen regelten.

61 Vgl. Li Hsin-ta 1995, S. 250f. und 267-304.
} 
einrichtungen, die eine Reihe von kaiserlichen Beamtenprüfungen koordinierten.

Es gab auch private konfuzianische Schulen für Schüler im Anschluss an die elementare Bildung, die sog. „Akademien“ (Shu-yuen). Sie entstanden mit der Verbreitung des Buddhismus in dem 6. Jahrhundert. mit Hilfe der freien und privaten Unterrichten während der Südlichen Song-Dynastie. ${ }^{62}$ Anzahl der Shu-yuen war 500 Ende Song-Dynastie, 2000 Ende Ming-Dynastie, über 4000 Ende Ching-Dynastie. ${ }^{63}$ Oft waren sie als wohltätige Stiftungen errichtet worden. Manche dieser Schulen hatten sogar eigene Ackerflächen und konnten sich dadurch eigenständig versorgen. Zunächst versuchten die konfuzianischen Gelehrten die Freiheit der Lehre und Forschung zu verwirklichen, ${ }^{64}$ später jedoch, während der Ming- und Ching-Dynastie, reduzierte sich ihre Rolle auf die Vorbereitung zur kaiserlichen Beamtenprüfung, sie standen somit unter der Aufsicht des Staates. ${ }^{65}$

\subsection{Wirkung des kaiserlichen Beamtenprüfungssystems}

Nach Konfuzianismus unterscheidet der Gebildete sich vom Ungebildeten, der einer „niederen Tätigkeit““, z. B. in Handwerk und Technik, nachgehen. Auf diesem Grund lehnten es die konfuzianischen Edler ab, zum Fachtechniker auszubilden und sich für weltliche Dinge zu interessieren. Die von Kaiserreich kanonisierten Lehrinhalten und Schreibstill in den Beamtenprüfungen bildeten die gemeinsame Sprache und politische, soziale, kulturelle und moralische Identität der Gelehrten. Dadurch wurde der Unterschied zwischen Gebildeten und Umgebildeten deutlich ausdifferenziert. Daher unterstüzte die kaiserliche Beamtenprüfung nicht nur die Auswahl der Beamten,

\footnotetext{
${ }^{62}$ Vgl. Linda Walton 1989, S. 457-492.

63 Vgl. Bai Hsi-liang 1995.

${ }^{64}$ In „China's University 1895-1995“ (1996) hat Ruth Hayhoe die unterschiedlichen Funktionen in der Bildung zwischen „Akademien“ (Shu-yuen) und Universitäten in Europa behandelt.

65 Vgl. Teng Hung-po 2005 und Huang Guang-liang 1976.
} 
legitmierte sondern auch die Ungleichheit der sozialen Schichten in der alten chinesischen Gesellschaft: Der Gelehrte besaß den höchsten Rang innerhalb der gesellschaftlichen Hierarchie, gefolgt von den Bauern, den Handwerkern und schließlich den Händlern und Kaufleuten auf der untersten Stufe. ${ }^{66}$

Obwohl konnte das kaiserliche Beamtenprüfungssystem die soziale Mobiltäten in der Tat nicht sehr stark betrieben, aber die Massen der Prüfungsausfallenden entwickelten eine soziale Schichte der Gebildeten, die sich mit ihrer reichend Kulturtechnik und -kenntnissen in vielen Bereichen umsetzten konnten, wie als Hauslehrer oder Lehrer in den konfuzianischen Schulen, Artzt, Anwalt, Dichter, Maler, Erzähler, Anbeter in Familietemple usw.. ${ }^{67}$ Sie bildeten sich einen sogenannten „konfuzianische Literatenstand“ (Shih-dai-fu), der nicht von Herkunft oder Besitz, sondern von der Intelligenz des Individuums bestimmt war und mit hohem Ansehen verbunden war. ${ }^{68}$ So entstanden Spruchworte Werte und Normen, nach denen ,es keine wertvollere Sache für eine Person als den Erwerb von Kulturtechniken und -kenntnissen gibt“ im sehr verbreitenden Kinderbuch „Gedichtsammelung der Wunderkinder“. Die durch Bildung gewonnene soziale Anerkennung und der Stolz des Individuums wurden als erstrebenswert angesehen, während körperliche Arbeit verachtet und nichtliterarisches Wissen gering geschätzt wurde, hierauf begründete sich die Legitimation sozialer Ungleichheit.

Das stark kokurrierende kaiserliche Beamtenprüfungsystem gewann die Unterstützung des Kaierreichs und der Gelehrten, weil die polititsche Ideologie des Kaiserreichs durch die Erziehung und Bildung ausbreiten konnte, zugleich

\footnotetext{
${ }^{66}$ Iona Man-Cheong 2004.

67 Vgl. Ho Ping-ti 1965.

68 Vgl. Max Weber 1991, S.111-135 und Benjamin A. Elman 2000, S.167-170.
} 
ermöglichte es den sozialen Aufstieg durch Bildung und diente gleichzeitig der Legitimierung der hierarchischen Gesellschaft. ${ }^{69}$

Zwar wurde der Eifer der Bevölkerung in Richtung Bildung angeregt, zugleich jedoch das konfuzianische Ideal der Erziehung abgeschwächt, denn es gab nur wenige, die den Wunsch nach moralischer Läuterung und geistiger Erleuchtung als Motivation zum Lernen hatten. Die meisten lockte die Aussicht auf einen lukrativen Posten. ${ }^{70}$ In den Prüfungen wurden detaillierte Kenntnis der Klassiker sowie bestimmte stilistische Fertigkeiten geprüft, was sich acht Jahrhunderte lang weitgehend nicht änderte. Durch die kaiserliche Beamtenprüfung steht alle Intelligenz des Kaiserreichs zu staatlichen Diensten und auch unter Kontrolle des Kaisereichs. Mit der Kanonisierung der Prüfungsinhalte und des Schreibstils sollte zwar mehr Gerechtigkeit hergestellt werden, aber der Kenntnisumfang der Prüfungsteilnehmer wurde auf den vom Herrscher erwünschten Kanon gerichtet, ja gesteuert. Die Gelehrten neigten mehr und mehr dazu, das praktische Element der konfuzianischen Klassiker beiseite zu lassen, um sich als Kandidat für den Staatsdienst zu qualifizieren. Die entstandenen negativen Wirkungen dieser Entwicklung sowohl auf den Staat als auch auf das Individuum wurden im Laufe der Geschichte von vielen Gelehrten angeprangert.

So übten die Gelehrte nach verfallen des Ming Kaiserreichs, wie Huang Tsung-shi $(1610-1695)^{71}$ und Ku Yen-wu (1613-1682), ${ }^{72}$ die Kritik daran, dass sich die

\footnotetext{
${ }^{69}$ Dass es zur sozialen Mobilität in der chinesischen Geschichte beigetragen hätte, wird nach heutigen Untersuchungsergebnissen als relativ gering eingeschätzt. Die Debatte über den Beitrag des kaiserlichen Beamtenprüfungssystems zur Mobilität der Gesellschaft im traditionellen China siehe Edward A. Kracke 1947, S. 103-123; Ho Ping-ti 1959, S.330-359 und ders. 1965; Denis C. Twitchett 1965, S. 28-41; Benjamin A. Elman 2000 und John W. Chaffee 1995.

${ }^{70}$ Zudem ist fraglich, ob man durch die Prüfung wirklich die Persönlichkeit der Teilnehmer erkennen konnte. Vgl. Lee Thomas H.C. 1993, S. 265-294.

71 Huang Tsung-shi (1610-1695) war Naturalist, Mathematiker, politische Theoritiker, Historiker, und Philosoph während Ende der Ming-Dynastie und Beginn der Ching-Dynastie. Vgl. Lu Shu-huei 2006.

${ }^{72} \mathrm{Ku}$ Yen-wu (1613-1682) war Historiker, Philologe und Geographe. Er nahme an der Aktivität von
} 
Literaten die meiste Zeit dem Studium der Klassiker widmeten, was für das Bewahren des Vaterlandes vor dem Einmarch des Mandchu-Volks und die Entwicklung des Gemeinwohls keinerlei praktischen Nutzen besäße. ${ }^{73}$ Ähnliche Kritikpunkte und die Forderung finden sich vor allem seit Mitte des 19. Jahrhunderts, als das ChingKaiserreich unter der Herausforderung des westlichen Imperialismus stand. ${ }^{74}$

Die chinesische Weltauffassung und Gesellschaftsordnung wurden über Jahrtausende wenig geändert, dazu zählen auch die Inhalte von Bildung und Erziehung. Erste Kontakte mit der abendländischen und christlichen Kultur entstanden Mitte der MingDynastie (um 1600) durch die Ankunft zahlreicher jesuitischer Missionare. Aufgrund der unterschiedlichen Einstellung zu religiösen Ritualen verhängte Kaiser Yungcheng (1678-1735) jedoch ab 1723 ein Verbot der Missionsaktivität und erließ einen Vertreibungsbefehl für jesuitische Missionare. ${ }^{75}$ Anschließend wurde fast 120 Jahre lang der Austausch mit der abendländischen Kultur, die in der Zwischenzeit eine drastische Änderung durch die Entwicklung neuer Kenntnisse, Aufklärung und Industrialisierung durchlief, unterbrochen. ${ }^{76}$ Mit den dauernden Angriffen der Weltmächte ab 1839 wurden die konfuzianische Erziehung und das kaiserliche Beamtenprüfungssystem in China in starke Erschütterung versetzt. Die fortgeschrittenden Gelehrten nahmen die Kenntnisse der Wissenschaft aus Europa und den USA schnell auf und waren der Meinungen, dass die Verbreitung der

Anti-Manchu teil und wurde vom Ching-Kaiserreichs verhfatet. Vgl. Chen Ban-chen 1988.

73 Vgl. Li Hsin-ta 1995, S. 328-336.

${ }^{74}$ Die Prüfungsform des gegliederten Aufsatzschreibens bzw. das kaiserliche Beamtenprüfungssystem verhinderten die Übernahme der „neuen“ bzw. „westlichen“ Kenntnisse; diese Meinung hatten Feng Kui-fen und Cheng Kuan-ying schon vor der Niederlage des Opiumkrieges geäußert. Nach dem sino-japanischen Krieg forderten auch Gelehrte wie Chang Chih-tung und Kang You-wei die Abschaffung. Vgl. Chen Chun-hua 2008, S. 7-9 und Rainer Hoffmann 1980, S. 83.

${ }^{75}$ Die Missionare wollten die Ahnenverehrung verbieten, ein wichtiger Teil der chinesischen Tradition. Die ideologischen Konflikte verschärften sich ab 1704, und ab 1723 wurden das Aktivitätsverbot und der Befehl zur Vertreibung der jesuitischen Missionare in China verhängt. Vgl. Gu Schang-sheng 2004, S. 11-15.

${ }^{76}$ Vgl. Benjamin A. Elman 2006, S. 13-35. 
Wissenschaftskenntnissen zur Aufklärung der chinesischen Gesellschaft. Am Ende des Ching-Kaiserreichs wurde das kaiserliche Beamtenprüfungssystem im Anschluss an das Schuledikt von 1903/04 im Jahr 1905 aufgehoben und damit die Fortsetzung der konfuzianischen Erziehung und Bildung infrage gestellt. ${ }^{77}$ Weiter kam es zu einer Kontroverse um die Gestaltung eines modernen, staatlichen Bildungswesens. Zudem wird es in dem Abschnitt 5 behandeln.

77 Vgl. Kuo Shu-ying 2002, S. 90-112. 


\section{Entwicklung der Erziehung auf Taiwan von 1626 bis 1895}

Zwar liegt die Insel Taiwan nah vom chinesischen Festland, jedoch war sie zu den weniger beachteten Teilen des chinesischen Kaiserreichs. Mit der Entdeckungsfahrt der Europäer seit Ende des 16. Jahrhunderts trat die Insel Taiwan wegen ihrer geographischen Lage auf die Weltgeschichte. Aufgrund des Machtwechsels in chinesischem Festland fluchten die besiegeten Beamten nach der Insel Taiwan und danach beherrschte der neue Sieger, das Ching-Kaiserreich über Taiwan. Seitdem immer mehr die Han-Chinesen nach der Insel Taiwan überwandelten, zugleich war die taiwaneseische Gesellschaft unter dem Einfluss der chinesischen Kultur. Wie die Erziehung auf Taiwan unter den Machtweschel entwickelte, behandelt dieser Abschnitt.

\subsection{Erziehung unter der Besatzung der Spanie und Niederland von 1626 bis 1662}

Im Jahre 1590 wurde die Insel Taiwan von portugiesischen Seefahrern „entdeckt“ und mit dem Namen Ilha Formosa (wunderschöne Insel) versehen. Mit der Ausbreitung der Entdeckungsfahrt wurde die Insel Taiwan als Basis der Transport der Europäer in Ostasien ausgewählt. Von 1626 bis 1642 hatten die Spanier Nordtaiwan besetzt, doch wurde Südtaiwan von 1624 bis 1662 von der „Dutch East India Company“ kontrolliert. Während dieser Zeit bemührteten sich katholische (in Nordtaiwan) aus Spanie und calvinistische (in Südtaiwan) aus Niederland Missionare, die Taiwanesen durch eine Bildungskampagne zum Christentum zu bekehren. ${ }^{78}$

\footnotetext{
78 Vgl. Tsao Yung-hen 1979, S. 25-44; Willim Campbell 2001 und Tonio Andrade 2007, S.162-378.
} 
3.2. Erziehung unter dem Ming- und Ching-Kaiserreich von 1662 bis 1894 auf Taiwan

Seit der Mitte des 17. Jahrhunderts wanderten immer mehr Chinesen nach der Insel Taiwan. Die taiwanesische Gesellschaft wurde dadurch unter der chineseischen Kultur geprägt. Wie die Erziehung unter dem Ming- und Ching-Kaiserreich von 1662-1894 auf Taiwan entwickelt wurde, stellt dieser Abschnitt vor.

\subsubsection{Begann mit der Errichtung der konfuzianischen Bildungseinrichtungen von 1662 bis 1684}

1644 beherrschte das Mandschu-Volk China und das neue Kaiserreich - ChingDynastie (1644-1911) - etabliert. 1662 floh Chen Cheng-kung (1624-1662), ein chinesischer General des Ming-Kaiserreichs, mit etlichen Beamten nach Taiwan, um dort eine Basis für die Rückeroberung des Festlands gegen das neu gegründete Ching-Kaiserreich zu errichten.

Seit 1662 begannen die geflohenen Loyalisten des Ming-Kaiserreichs das Bildungssystem nach dem Vorbild des Ming-Kaiserreiches auf Taiwan auszubauen, langsam verloren die von der holländischen und spanischen Kirche gegründeten Missionsschulen ihren Einfluss. ${ }^{79} 1666$ wurde der erste konfuzianische Tempel in Taiwan errichtet. In dem wurde kaiserliche Schule für höhere Studien und zur Ausbildung der höheren Beamten (Tai-Hsueh) sowie die konfuzianischen Schulen für elementare Erziehung und Bildung, in denen die Kinder und Jugendliche in chinesischer Sprache und Kultur unterrichtet wurden, gestaltett. Die Lehrinhalte waren denen auf dem chinesischen Festland sehr ähnlich. Zudem wurde das administrative System für zentrale und locale Bildung aufgebaut.

\footnotetext{
79 Vgl. Yen Hsien-chun 1996, S. 88-90 und Hsu Nan-hao 1996, S. 214.
} 


\subsubsection{Erweiterung der Erziehungseinrichtungen unter dem Ching-Kaiserreich von 1684 bis 1895}

Die Ming-Loyalisten wurden jedoch 1682 von der Mandschu-Dynastie vertrieben, anschließend wurde 1684 die Insel Taiwan dem Ching-Kaiserreich als eine Präfektur der Provinz Fuchien zugefügt. ${ }^{80}$ Anhand der Statistik gab es 1684 50-70 Tausende Einwohner auf Insel Taiwan. Die Zuwanderungsströme aus den Südprovinzen des chinesischen Festlands ließen die Bevölkerungszahl in den nachfolgenden 212 Jahren anwachsen, vor der Abgabe an japanischer Herrschaft (1895) gab es ca. 2,5 Millionen Einwohner auf Taiwan. ${ }^{81}$ Inzwischen wurden zahlreiche chinesische Systeme auf Taiwan umgesetzt. Das kaiserliche Beamtenprüfungssystem war eins davon. Der aufgenommen Anzahl der Kandiaten in der kaiserlichen Beamtenprüungen wurden langsam erhöht - z. B. bis 1889 wurden die Anzahl der aufgenommen Kandiaten, Hsiu-tsai (zu Deutsch „ausgezeichnetes Talent“ oder „ausgesuchter Schüler“) für die erste Stufe der kaiserlichen Beamtenpürung auf 208 erhöht. ${ }^{82}$ Das Bildungswesen nach dem Vorbild des chinesischen Festlands wurde im Laufe der Zeit erweitet. Nach den über zwei Hundert Jahren Herrschaft des Chinesischen Kaiserreichs wurden die konfuzianische Erziehung und Schule in Taiwan gut verbreitet. In den 1890er-Jahren gab es 13 amtliche konfuzianische Schulen für die höhere Bidlung zur Ausbildung der Beamten und 63 „Akademien““ ${ }^{83}$ außerdem 273 ,amtliche Gemeindeschulen“, 83 „Sponsoren schulen“ und 1,127 „Studienzimmer“. ${ }^{84}$

\footnotetext{
${ }^{80}$ Vgl. Kao Ting-i 1988, S.82f.

${ }^{81}$ Vgl. Chang Hai-pung und Tao Wen-chao 2010, S.33.

${ }^{82}$ Vgl. edb. S.58.

${ }^{83}$ Vgl. Lai An-ming 1996, S. 135.

${ }^{84}$ Vgl. Tseng Hui-wen 1999, S. 19-305 und Chiu Hei-yuen 2003, S. 1-39.
} 


\subsection{Annäherung an westliche Erziehung in Taiwan}

Ende der Herrschaft des chinesischen Kaiserreichs wurde die westliche Form der Ausbildung in Taiwan eingeführt. In diesem Abschnitt werden der Hintergrund der Annäherung an westliche Erziehung und der Erster Versuch für die Errichutng der westlichen Bildungsformen von 1885 bis 1894 behandeln.

\subsubsection{Beginn mit der Einführung der westlichen Bildungsformen innerhalb der „Selbststärkungsbewegung““ im Ching-Kaiserreich von 1862 bis 1894}

Laut Vertrag von 1860 - nach dem Sieg von Briten und Franzosen im zweiten Opiumkrieg - wurde allen protestantischen und katholischen Missionaren in China völlige Bewegungs- und Betätigungsfreiheit zugesichert. ${ }^{85} 1868$ zwang die US-Regierung - obwohl sie an den zweiten Opiumkrieg nicht teilgenommen hatte das Ching-Kaiserreich sogar dazu, eine Genehmigung für die Errichtung von Missionsschulen zu unterzeichnen, ${ }^{86}$ welche dann Schritt für Schritt der westlichen Form von Grundschule, Mittelschule und Hochschule angepasst wurden. Neben christlichen Werten wurde hier Mathematik, Physik, Chemie, Geographie, Medizin, westliche Geschichte und Rechtslehre unterrichtet. Um die westlichen Kenntnisse in China zu verbreiten, veröffentlichten die Missionare Zeitungen, übersetzten Bücher ${ }^{87}$ und waren auch aktiv in der Selbststärkungsbewegung, z.B. die Timothy Richard (1845-1919) und Alexander Wylie (1815-1887) aus Großbritannien sowie William A.

\footnotetext{
${ }^{85}$ Vgl. Jürgen Osterhammel 1989, S. 149.

${ }^{86}$ Die christlichen Missionsschulen breiteten sich in China sehr rasch aus. Bis 1898 gab es 1032 Missionsschulen für die elementare Erziehung mit 16.310 Schülern, 74 für die Mittelstufe mit 3819 Schülern sowie einige Bildungseinrichtungen für die höhere Bildung. Vgl. Chen Fang-ping und Bi Chen 1996, S. 328. Gemäß der Untersuchung von „The China Christian Educational Association“ gab es im Jahr 1918 folgende von christlichen Missionaren gestaltete Erziehungseinrichtungen: 15 Kindergärten mit 3196 Kindern, 5276 Grundschulen mit 133.826 Schülern, 575 höhere Grundschulen mit 19.605 Schülern, 233 Mittelschulen mit 12.533 Schülern, 28 Fachschulen mit 1499 Schülern, 40 Berufsschulen mit 1409 Schülern und 13 Universitäten mit um 18.000 Studenten sowie andere Erziehungseinrichtungen, z.B. Waisenhäuser. Vgl. Li Chih-kang 1992, S. $211 \mathrm{f}$.

${ }^{87}$ Vgl. Benjamin A Elman 2005 und 2006.
} 
P. Martin und Young John Allen aus den USA. ${ }^{88}$

Nach der Niederlage im zweiten Opiumkrieg gegen Großbritannien und Frankreich sowie die Niederschlagung des Taiping-Aufstands ${ }^{89}$ in Südchina wurde 1862 die sogenannte „Selbststärkungsbewegung“ unter Leitung von Prinz Kung I-hsin (18331898) und den Beamten Tseng Kuo-fan (1811-1872) und Li Hung-chang (1823-1901) gestartet. $^{90}$ Deren besonderes Interesse lag in der Annäherung an technische und naturwissenschaftliche Kenntnisse des Abendlandes sowie der Beschaffung militärischer Rüstung durch Vermittlung der christlichen Missionare. In der Folge wurden drei Arten von Fachschulen errichtet: Fremdsprachen- ${ }^{91}$, Technik- ${ }^{92}$ und Militärschulen. ${ }^{93}$ Dies waren unabhängige Bildungseinrichtungen, d. h., sie übernahmen die westlichen Schulformen ohne Rücksicht auf die Verbindung zu anderen Bildungsstätten. Außerdem gab es nur wenige Plätze ${ }^{94}$ in diesen Schulen, sie konnten daher das traditionelle Erziehungssystem nicht beeinflussen. Um Schiffbau, Schifffahrt und Militärtechnik zu lernen, wurde ein Teil der Schüler nach England, Frankreich und Preußen entsandt. ${ }^{95}$ Zwischen 1872 und 1875 wurden zudem viermal

\footnotetext{
${ }^{88}$ Vgl. Lee Han-yun 2003, S. 55-58.

${ }^{89}$ Der Taiping-Aufstand in Südchina (1850-1864), eine antimandschurische, religiöse und soziale Rebellion gegen das Ching-Kaiserreich unter Führung von Hung Hsiu-chuan, hatte den Aufbau der Sozialutopie des Konfuzianismus zum Ziel: die ,große Gemeinsamkeit der Welt“. Mithilfe britischer und US-amerikanischer Truppen wurde der Aufstand niedergeschlagen. Vgl. Teng Ssu-Yu 1971 und Lee Han-yun 2003, S. 33-43.

90 Vgl. Kuo Ting-i 1987.

91 Die erste Fremdsprachenschule in Peking von 1862 und ähnliche in Shanghai und Kuangtung dienten dazu, Übersetzer für internationales Recht, internationale Beziehungen und Diplomaten auszubilden. Später wurde die Übersetzung der westlichen Kenntnisse in Astronomie, Mathematik, Physik, Chemie, Medizin, Botanik, Geographie, Geologie, Maschinenbau und Navigation mit einbezogen. Nur Kinder vom Mandchu-Hofe durften diese Schulen besuchen. Vgl. Li Hsi-suo 1987, S. 79-85; Benjamin A. Elman 2006, S. 100-131 und Yang Kuo-tzu 1981 (a), S. $726 f$.

${ }^{92} 1867$ wurde die Schiffbau- und Schifffahrtschule in Fuzhou, 1874 in Shanghai errichtet. Vgl. Chien Man-chien und Chin Lin-hsiang 1996, S. 42-45; Yang Kuo-tzu (a) 1981, S. $727 f$.

931881 Errichtung der Militärschulen in Tienchin und 1886 in Kuangtung. Vgl. Chien Man-chien und Chin Lin-hsiang 1996, S. 45-47; Yang Kuo-tzu (a) 1981, S. 727.

${ }^{94}$ Z.B. gab es in der Schiffbau- und Schifffahrtsschule in Fuchien von 1866 bis 1907 nur 628 Absolventen. Vgl. Chien Man-chien und Chin Lin-hsiang 1996, S. 33-35.

95 Vgl. Li Hsi-suo 1987, S. 77-116.
} 
jeweils vierzig ausgesuchte Jungen im Alter zwischen zehn und vierzehn Jahren im Rahmen des Programms „Chinese Educational Mission to the US“ in die USA geschickt. ${ }^{96}$ Die Schüler sollten vielmehr lediglich bestimmte Errungenschaften und Fertigkeiten der fremden Kultur erlernen, jedoch nicht westliche Mentalität und Benehmen. $^{97}$

\subsubsection{Konstruktion der Provinz Taiwan und Einführung der Modernisierungsprojekte im Rahmen der Selbststärkungsbewegung von 1885 bis 1894}

Nach dem ersten Opiumkrieg 1840 begehrten die Weltmächte wie Großbritannien, USA, Fankreich und Japan nicht nur die Häfen auf dem chinesischen Festland, sondern auch auf der Insel Taiwan zum Vertragshafen, nämlich freie Handelshäfen zu fordern. ${ }^{98}$ Unter den dauernden Angriffe und der Anforderung der Weltmächte auf die Häfen auf Taiwan nahmen die Beamten wie Shen Bao-chen (1820-1879) ${ }^{99}$ und Liu Ming-chuan (1836-1896) ${ }^{100}$ die militärischen und wirtschaftlichen Bedeutung der Insel Taiwan wahr, daher bemührten sie sich Taiwan zu eigenständiger Proviz zu konstituieren. ${ }^{101} 1885$ wurde Insel Taiwan zu zwanzigster Provinz des chinesischen

\footnotetext{
${ }^{96}$ Das Projekt wurde jedoch vorzeitig wieder abgebrochen, der Grund lag in der Verhaltensänderung der entsandten Schüler, denn diese wandten sich von der chinesischen Tradition ab, schnitten sich z. B. ihre Zöpfe ab, sympathisierten mit dem Christentum, trugen westliche Anzüge und widersetzten sich der Niederwerfung vor den Beamten, was diese nicht hinzunehmen bereit waren. Vgl. Sung Hsi 1985, S. 425-263.

${ }_{97}$ Vgl. Li Hsi-suo 1987, S. 50-55; Wolfgang Franke 1967, S. 70; Lai Hui-lan 1985 und Sung Hsi 1985, S. 425-463.

${ }^{98}$ Z.B. Laut dem Vertrag Tenjin 1860 forderten nicht nur Großbritanien, Frankreich die Häfen in Taiwan wie Tamsui und Kaohsiung u.a. zu Vertragshäfen zu öffnen, sondern auch US-Amerika und Rußland, anschließend die anderen Länder wie Preußen(1861), Niederland und Dänemark (1863), Spanien (1864), Belgien (1865); Italien (1866), Österreich (1869), Japan (1871), Peru (1874) und Brasilien (1881).

${ }^{99}$ Shen Baozhen war ein der wichtigen Beamten innerhalb der "Selbststärkungsbewegung“ und für den Aufbau der modernen Schiffe und Schule der Schiffbau- und Schifffahrt in Fuzhou zuständig. 1874, als Japan Taiwan angeriffen hatte, hatte Shen Taiwan verteidigt. Vgl. Chang Hsi-Hsien 2009, S.219-220

${ }^{100}$ Liu Ming-chuan war ein der wichtigen Offizier in den Krieg gegen Frankreich im Jahr 1884. Als er zum ersten Generalgouverneure der Provinz Taiwan berief und zum Modernisierung Taiwan groß beitrug. Vgl. Chang Shih-Hsien 2009, S.232-235.

${ }^{101}$ Vgl. ebd. S.215-224.
} 
Kaiserreichs erhoben. Seitdem die Modernisierungsprojete im Zusammenhang der „Selbststärkungsbewegung“ (1861- 1894) ${ }^{102}$ in Taiwan im Gang gesetzt wurden, d.h. die Einsetzung der militärischen Rüstungen und der Aufbau der wirtschaftlichen, politischen und erzierlichen Infrastruktur wurden gestärkt und verwestlicht bzw. modernisiert.

\subsubsection{Erster Versuch für die Errichutng der westlichen Bildungsformen von 1885 bis 1894}

Der erste Gouverneur, Liu Ming-chuan, bemührte sich nicht nur die Techniker aus den europäischen Ländern zur Durchführung seiner Modernisierungsprojekte wie Aufbau des Kommunikationssystems wie Telegramme und Post sowie des Verkehrsystems wie Eisenbahnaufbau in Taiwan einzuladen, sondern auch die Bildungseinrichtungen nach westlichem Vorbild $\mathrm{zu}$ gestalten, in den die entsprechenden Fachkräfte im Dienst seiner Modernisierungsprojekte auszubilden. 1887 wurde eine Einrichtung zur Ausbildung von Dolmetschern und Dipolmaten errichtet, in der die westlichen Lehrer die Fremdsprachen wie Englisch und Französische, westliche Geographie, Geschichte, Mathematik, Messung, Physik und Chemie einerseits, andererseits die chinesischen Lehrer die chinesischen Klassiker unterrichteten. 1890 wurde eine Einrichtung zur Ausbildung von Fachkräften für Telegrafie errichtet, derer Schüler von Einrichtung zur Ausbildung von Dolmetschern und Dipolmaten ausgesucht wurden. 1890 nach Lius Abtreten kehrte man wegen der Mangel an ausreichenden Mittel langsam zum alten Konzept zurück. ${ }^{103}$ Obwohl die beiden westlichen Ausbildungseinrichtungen in Taiwan nur für kurze Zeit dauerten und es lediglich von 64 Schülern gab, aber es galt als das erste Modernisierungsprojet

\footnotetext{
102 Vgl. 3.3.1. in dieser Arbeit.

103 Vgl. Tai Pao-tsun 2006, S. 177f.
} 
der Erziehung, die westlichen Kenntnisen in den amtlichen Bildungseinrichtungen zu unterrichten und eine Verbindung zwischen des staatlichen Aufbaus und beruflichen Ausbildung zu ermöglichen. Nach der Ankuft der japanischen Kolonialherrschaft, 1895 wurde das Projekt für Annäherung an den westlichen Bildungsformen weiter und breiter fortgesetzt. 


\section{Aufbau des modernen Bildungssystems unter der japanischen Kolonial- herrschaft von 1895 bis 1944}

Mit der Niederlage des Kaiserreichs im Sino-Japanischen Krieg (1894-1895) musste das Ching-Kaiserreich die Insel Taiwan sowie die Pescadoren an Japan abtreten. In der Folgezeit wurde ein modernes staatliches Bildungssystem in Taiwan eingeführt. In diesem Abschnitt soll die Bidlungspolitik und der Aufbauprozess des modernen Bildungssystems unter der japanischen Kolonialherrschaft von 1895 bis 1944 untersucht werden.

\subsection{Japanische Bildungspolitik im 19. Jahrhundert}

Im Laufe des 19. Jahrhunderts strebten die Japaner nach Europäisierung, im Zuge der sog. „Meiji-Restauration (1868-1912)“،104 nahmen sie vor allem Preußen zum Vorbild für wirtschaftliche Entwicklung und Stärkung des Militärs, und Japan wurde zum ersten modernen Industriestaat in Asien. Die kaiserliche Verfassung von 1889 postuliert - in starker Anlehnung an die preußische Verfassung von 1850 - einen starken Obrigkeitsstaat mit dominanter Rolle des Militärs als Damm gegen liberale und demokratische Bestrebungen.

Währden der „Meiji Restauration“ wurden zahlreiche westliche moderne Erziehungskonzepte in Japan eingeführt, wie die Werke der Pädagogen, D.P. Page (1810-1848), Herbert Spencer (1820-1903), Johann Heinrich Pestalozzi (1746-1827) und Johann Friedrich Herbart (1776-1841) usw. ins japanische übersetzt wurden und einen starken Einfluss auf der japanischen Bildung und Erziehung bis 1930er-Jahren

\footnotetext{
104 Die „Meiji Restauration“ bezeichnet den politischen Umbruch im Jahr 1868 und den Beginn einer politischen Reform unter Leitung des Meiji-Kaisers. Die Bewegung vollzog, wie der bedeutende Denker, Fukuzawa Yukichi (1835-1901) konstatierte, „die Abkehr Japans vom Rest Asiens und die Integration in Europa“. Vgl. Bernd Martin 1987, S. 17-44.
} 
hatten. ${ }^{105}$ Im Zuge der Verwestlichung wurden auch die Ideen eines allgemeinen und staatlich kontrollierten Bildungswesens übernommen, um die Aufklärung der Bevölkerung zu mündigen Staatsbürgern voranzutreiben und eine nationale Identität auszubilden. 1871 wurde das Kulturministerium zur Administation des staatlichen Bildungswesen errichtet, anschließend wurde 1872 ein Entwurf für Aufbau des Volksbildungswesens anhand den Modelle von England, Niederland, US-Amerika und Preußen erstellt. ${ }^{106}$ Am 30. Oktober 1890 erließ der erste Präsident vom Beratungsgremium des japanischen Kaisers (1890-1891), Graf Itō Hirobumi (18411909), ${ }^{107}$ ein Schuledikt, das einen Kompromiss bildete zwischen westlichen Bildungskonzepten - vor allem Patriotismus und Militarisierung - zum einen und konfuzianischer Morallehre und dem traditionellen Geist Japans, Shintoismus, zum anderen. Beide Ideale begleiteten den Aufbau des modernen Bildungswesens in Japan von den 1890er-Jahren bis zum Zweiten Weltkrieg. ${ }^{108}$ Das reformierte Kaiserreich übernahm nach westlichem Vorbild die Verwaltung sowie Gestaltung der Schulen. Zugleich wurden Richtlinien geschaffen, nach denen sich der Schulalltag richten sollte. So wurde die Einführung bestimmter Schulfächer detailliert geplant. Neben der Beherrschung von Lesen, Schreiben und Rechnen wurden eine einheitliche nationale Verkehrssprache sowie die Fächer Geschichte und Geographien des Japans und der Welt eingeführt, damit die Schüler ein nationales Zugehörigkeitsgefühl entwickeln konnten. Des Weiteren wurden Sport und Hygiene im Lehrplan aufgenommen, um die physische Kondition der Bevölkerung zu verbessern. Hinzu kamen naturwissenschaftliche Fächer wie Physik, Chemie und Geometrie. ${ }^{109}$ Aufgrund der

\footnotetext{
${ }^{105}$ Vgl. Chu Je-ying 2011, S. $23-27$.

${ }^{106}$ Vgl. Chu Je-ying 2011, S. $17 \mathrm{f}$.

${ }^{107}$ Ito war ein bedeutenster Politiker und war der erste und auch viermal Premierminister Japans (1885-1888, 1892-1896, 1898-1898 und 1900-1901) Ku Ming-yuen 2000, S. 194-198.

108 Vgl. Ku Ming-yuen 2000, S. 572.

109 Vgl. Horst E. Witting 1976, S. 89f.; Teruhisa Horio 1998, S. 305-322, und Volker Schubert 1998, S. 323-337.
} 
Mobilisierung aller Bevölkerung zur Militarisierung wird die Schulpflicht für Elementarbildung ab 1900 eingeführt. ${ }^{110}$

\subsection{Bildungspolitik unter der japanischen Kolonialherrschaft}

Als Kolonialherrscher war Japan noch unerfahren. Nach der Landung der Truppen war der bewaffnete Widerstand gegen die Besatzungsmacht stark, und es stellte sich die Frage, ob Japan seine Kolonie wirksam kontrollieren konnte und wie Taiwan regiert werden sollte. Die verschiedenen Standpunkte hierzu können grob in zwei Sichtweisen unterteilt werden:

Der Mediziner Gotō Shimpei (1857-1929) ${ }^{111}$ vertrat die durch britisches Kolonialdenken von Darwin's ,the origin of species“ beeinflusste Ansicht, dass die Ureinwohner in den Kolonien aufgrund anderer „biologischer Eigenschaften“ nicht vollkommen assimiliert werden könnten, sie seien daher anders zu regieren als die Menschen in Japan und müssten einen völlig neuen Gesetzeskatalog erhalten. Der Pädagoge Izawa Syuji (1851-1917) $)^{112}$ und später auch Premierminister Hara Takashi (1856-1921) $)^{113}$ gingen hingegen davon aus, dass die Taiwanesen ähnliche Werte, Normen sowie einen vergleichbaren Lebensstil wie den Japaner hätten, was auf konfuzianischen Einfluss zurückgehe. Taiwan sei deshalb als direkte Verlängerung

\footnotetext{
${ }^{110}$ Vgl.Wang Chih-hsiang 2008, S.89-92.

111 Gotō Shimpei hatte von 1890 bis 1892 Medizin in Deutschland studiert, sein Vorbild war Otto von Bismarck. Er war von 1898 bis 1906 Direktor der Administration für innere Angelegenheiten (Zivilgouverneur) im Gouvernement Taiwan, danach erster Präsident der südmandschurischen Eisenbahn sowie der Pfadfinderverein in Japan, Minister für Unterhaltung, Politiker in verschiedenen Kabinetten und später Bürgermeister von Tokio.

${ }^{112}$ Izawa Syuji war der erste japanische Pädagogikstudent an der Bridgewater Normal School in Massachusetts, USA, und mit dem Entwurf eines Erziehungskonzepts für die Volksbildung durch das staatliche Bildungssystem vertraut. Er amtierte von 1895-1897 als Bildungsminister auf Taiwan. Als er nach Japan zurückkehrte, beschäftigte er sich mit moderner Musikerziehung und Erziehung für blinde und taube Kinder. Vgl. Chu Je-ying 2011, S. 28-36.

${ }_{113}$ Hara Takashi, der erste nicht adlige Premierminister in der japanischen Geschichte (1918-1921) und Premier des ersten reinen Parteienkabinetts, erlangte Berühmtheit durch die Einführung des parlamentarischen Systems in Japan.
} 
Japans zu betrachten und relativ gleich zu behandeln. Durch die Erziehung sollten die Bürger dann langsam den Japanern angeglichen werden. ${ }^{114}$

Die Kontroverse zog sich durch die gesamte Kolonialzeit, auch in der Bildungspolitik spielte sie eine Rolle, speziell in der Frage, wie das moderne Bildungssystem in Taiwan aufgebaut werden solle. Hier verfolgte Japan verschiedene Strategien. Während der 50-jährigen Kolonialzeit herrschten 19 Generalgouverneure über Taiwan, von 1895 bis 1918 sieben Militärgeneräle, von 1919 bis 1936 neun Zivilbeamte und schließlich von 1936 bis 1945 wieder drei Militärgeneräle. ${ }^{115}$

Mit dem Herrschaftswechsel ging auch eine veränderte Kolonialpolitik einher. Von 1895 bis 1918 versuchten die Generalgouverneure mit starker Militär- und Polizeipräsenz die dauernden Widerstände der Taiwanesen zu unterdrücken, zugleich verfolgten sie eine behutsame Herrschaftspolitik nach dem Prinzip des „Gradualismus“ von Gotō Shimpei. Die japanische Regierung beeilte sich also nicht mit Integrationsmaßnahmen, sondern untersuchte zunächst die Sitten und Gebräuche des Landes, um auf ethischer Basis die Regierungspolitik zu erstellen. Das neu eingeführte moderne Bildungssystem im elementaren Bereich sollte dabei vor allem zur Verbreitung der japanischen Sprache und Kultur dienen, um die Kommunikation zwischen Taiwanesen und Japanern zu ermöglichen.

\footnotetext{
114 In jener Zeit gab es in Japan zwei akademische Standpunkte zur Staatskörperlehre: Die einen waren der Meinung, dass Japan aus vielen Völkern bestehe, welche wie innerhalb einer Großfamilie unter dem japanischen Kaiser solidarisch lebten. Die fremden Völker in der Kolonie können als Adoptivkinder in die Großfamilie integriert werden, sofern sie auf ihre eigene Kultur verzichten und sich der japanischen Kultur angleichen. Die anderen sahen die Japaner als homogenes Staatsvolk und lehnten eine ethnische Mischung ab. Vgl. Nobuo Fujikawa 2006, S. 45-66.

115 Vgl. Wang Chin-chueh 2002, S. 131-158.
} 
Innerhalb der Debatte warnten einige Stimmen davor, dass die Einführung einer höheren Bildung zur Aufklärung führe, indem sie das Selbstbewusstsein der Menschen in den Kolonien stärke, was wiederum mit der Kritik an der japanischen Fremdherrschaft einhergehen und eine Forderung nach Selbstbestimmung und die Destabilisierung der Kolonialmacht nach sich ziehen könne. ${ }^{116}$ „Die Plätze für eine höhere Bildung anzubieten ist absolut keine kluge Sache“, widersprach der Direktor der Administration für innere Angelegenheiten im Gouvernement Taiwan (19101915), Uchida Kakichi (1866-1933), den Reformplänen und brachte damit die Meinung des größten Teils der japanischen Beamten und Gelehrten zum Ausdruck. Vielmehr sei die berufliche Ausbildung und die Vermittlung von praktischen Fertigkeiten zu fördern, damit sich die Kolonialisierten „im Leben zurechtfinden können““. ${ }^{117}$ Diese Bedenken führten dann zu einem sehr auf Praxis ausgerichteten und militarisierten Bildungswesen, zugleich wurden die japanischen und taiwanesischen Kinder aufgrund der Separationsmaßnahme in der Schulerziehung ungleichbehandelt.

Erst nach dem Ersten Weltkrieg bekamen die Befürworter einer Assimilationspolitik mehr Unterstützung seitens der japanischen Gesellschaft und Regierung. ${ }^{118}$ Taiwan sollte von einem Überwachungs- zu einem Verfassungsstaat umgewandelt, genauer: in die Pläne zur Schaffung eines „Großjapanischen Wirtschaftsraumes“ als Erweiterung des japanischen Kaiserreichs integriert werden, um als „Basis für den Sprung nach Süden“ der nach Südostasien gerichteten Politik und der Ausdehnung

\footnotetext{
116 Als Beispiele für derartige Entwicklungen dienten Indien und die Philippinen, wo die Forderung nach mehr Selbstbestimmung der Völker und der Widerstand gegen die Kolonialherren nach dem Ausbau des Schulwesens stark zunahmen. Vgl. E. Patricia Tsurumi 1977, S. 45-48; Tsai Pei-huo 1971, S. 36 und Wu Wen-hsing 1992, S. 98.

117 Zit. nach E. Patricia Tsurumi 1977, S. 47f. Eigene Übersetzung vom Englischen ins Deutsche.

118 Vgl. Yanaihara Tadao 1987, S. 176-180.
} 
des japanischen Imperialismus zu dienen. Die Strategie der Wirtscahftsentwicklung wird auch ab 1930 von agrawirtschaftlicher Produktion zur Industrialisierung umgestellt. In der Phase, von 1919 bis 1936, versuchten die Generalgouverneure das Bildungssystem an Japan anzugleichen und Bildungsangebote in der sekundären und tertiären Stufe für taiwanesische Jugendliche zu schaffen. Dadurch sollten die für die Industriealisierung benötigen Arbeitskräfte und die Forschungskräfte für Untersuchung über Südostasien ausgebildet werden.

Während des Zweiten Weltkriegs in Ostaisen bzw. Sino-Japansichen Kriegs (19371945) richtete sich die schulische Erziehung am Militarismus aus, um die Taiwanesen zu Untertanen des japanischen Kaisers zu erziehen. Ab 1942 wurden immer mehr Jugendliche dazu angeregt, als Kriegsfreiwillige in die Kaiserliche Japanische Armee oder Marine einzutreten, und schließlich 1944 zum Wehrdienst berufen. ${ }^{119}$ Darüber hinaus wurde die Bewegung der Japanisierung vorangetrieben. D.H., dass die Taiwanesen auf ihren Glauben verzichten, stattdessen die japanischen Götter und den Kaiser verehren und sich den Sitten und Gebräuchen anpassen sollten, z.B. japanische Kleidung tragen und in japanisch gebauten Häusern wohnen. Chinesische Zeitungen wurden verboten, um die Verbindung zu China und zur chinesischen Kultur zu unterbrechen. Und die Einheimischen sollten in der Öffentlichkeit Japanisch und kein Chinesisch und Taiwanesisch sprechen. Familien, die sowohl in der Öffentlichkeit als auch im privaten Bereich die japanische Sprache anwandten, wurde ein Zeugnis verliehen, das ihnen einige soziale Privilegien sicherte, so durften ihre Kinder die Grund- und Mittelschule für japanische Jugendliche besuchen, hatten mehr Aussichten auf amtliche Stellen, erhielten größere Essensrationen usw. 1940 wurden

119 Vgl. Chen Hsiao-chung 2005, S. 303-311; He I-lin 1986 und Oskar Weggel 1991, S. 72-88. 
Gesetze verabschiedet, die bestimmten, dass die Taiwanesen auf ihren chinesischen Namen verzichten und die japanische Form des Namens benutzen sollten. ${ }^{120}$

\subsection{Aufbauprozess des modernen Bildungssystems}

In diesem Abschnitt wird der Aufbau der primären, sekundären und tertiären Stufe im Bildungswesen unter der japanischen Besatzung vorgestellt.

\subsubsection{Die Primarstufe: Elementarbildung und öffentliche Schule}

1895 trat Izawa Syuji den Dienst als erster Bildungsminister des Gouvernement Taiwans an. Taiwan diente fortan als Versuchsfeld seines Erziehungskonzeptes mithilfe eines staatlichen Bildungssystems nach westlichem Vorbild. Er war der Meinung, dass die japanische Regierung Taiwanesen nicht nur durch die Waffen erobern sollte, sondern auch durch den Geist kultivieren sollte. D.H., dass mithilfe der Bildung des Geistes die geistigen Verbindung der Taiwanesen mit dem China abgebrochen sollte und die Taiwanesen zu richtigen japanischen Bürger umgebildet werden sollten. Daher sollte die japanische Regierung die Aufgabe für Erziehung der Taiwanesen übernehmen und die Kontrolle über die Bildung und Erziehung ausüben sollte. ${ }^{121}$ Die Entwicklung der Bildungspolitik für die elementare Erziehung unter der japanischen Besatzung ist folgend.

\subsubsection{Einführung der öffentlichen Schule}

1895 errichtete er in Taipei die erste „Landessprachliche Lehranstalt“ zur elementaren Erziehung, in der die japanische Sprache gelehrt wurde, später kamen klassisches

\footnotetext{
${ }^{120}$ Vgl. Hung Hsuan-fan, 1994; Li Hsiao-feng und Liu Feng-sung 1994, S. 149.

121 Vgl. Chu Je-ying 2011, S. 29f.
} 
Chinesisch, Arithmetik, Musik, Gesundheitswesen und militärische Gymnastik hinzu. 1896 wurde die „Landessprachschule“ in „Landessprachliche Lehranstalt“ umbenannt. Da die Resultate zufriedenstellend waren, befahl der vierte Generalgouverneur, Kodama Gentarō (1898-1906), 1898 die Einrichtung von sechzehn weiteren Lehranstalten, so für japanische Beamte, Soldaten und Polizisten zum Zweck der Aneignung des Taiwanesischen. Hinzu kamen Grundschulen für japanische Kinder, die mit ihren Eltern, Beamte, Lehrer und Militärs, nach Taiwan übersiedelt waren.

Im Juli 1898 wurde die „Regelung für die öffentliche Schule in Taiwan“ zur Verbreitung der japanischen Sprache und Kultur und zur Ausbildung der Berufstätigkeit in Taiwan verkündet. Die elementare Bildung wurde nach ethnischer Zugehörigkeit in drei Schulformen mit unterschiedlichen Lehrplänen und Lehrinhalten gegliedert: die sechsjährige „primäre Schule“ für japanische Kinder, die sechsjährige „öffentliche Schule“ für taiwanesische Kinder und eine vierjährige Schule für die Kinder der Ureinwohner. ${ }^{122}$

Die „Landessprachlichen Lehranstalten“ waren also in „öffentliche Schulen“ für taiwanesische Kinder im Alter von acht bis vierzehn Jahren zum Zweck des Japanischlernens und der bürgerlichen Erziehung umgewandelt worden. In der öffentlichen Schulen wurde nur von japanischen Herrschaft anerkannten Werten und Normen sowie Kenntnisse systematisch vermittelt. Außerdem wurde die Bildung der Benehmen als japanischen Bürger und alltäglichen Gewohnheiten zur Lehrinhalt einbezogen. Die Schulfächer dienten als Mittel zur Assimilation, so etwa „Persönlichkeitspflege“ (Japanisch: shūshin, „Staatsbürgerkunde und moralische Instruktion"), in dem die Schüler die japanische Sitte und Normen sowie die ${ }^{122}$ Vgl. Yen Hsien-chun 1996, S. 96-99. 
erzieherischen Reden des japanischen Kaisers erlernen sollten, „Japanische Sprache“ in Form von Lesen, Schreiben und literarischem Schreiben, „Singen“ und „Sport mit japanischer Kampfkunst“ ${ }^{123}$ Die japanische Sprache war der wesentliche Lehrinhalt, daneben wurden unter westlichem Einfluss auch Kenntnisse in Arithmetik, Naturwissenschaften und Geographie vermittelt. Außerdem wurden die Abschlußreisen, Wettbewerb im Sport, Singen, Tanzen und Theater als Teil der schulischen Aktivitäten angetrieben. Die nach westlichen Standards ausgerichteten Lehrinhalte spiegelten die Forderungen des modernen Staates an seine Bevölkerung wider und verkörperten damit eine neue pädagogische Zielsetzung. Als polytechnische Fächer kamen Handwerk, Ackerbau, Fischerei, Buchführung und Grundzüge der Mathematik, Arithmetik sowie Nähen und Hausarbeit für Mädchen hinzu, wodurch die Jugendlichen in Arbeitstugenden wie Fleiß, Sparsamkeit, Gehorsam, Sorgfältigkeit, Sauberkeit, Hygiene herangebildet und zur Abkehr vom Aberglauben bewegt werden sollten. Zusätzlich wurden sie körperlich ertüchtigt. Durch die elementare Erziehung förderte die japanische Regierung eine Ausbildung für einfach qualifizierte Arbeitskräfte zum Zwecke des wirtschaftlichen und infrastrukturellen Aufbaus der Kolonie in den Sektoren Landwirtschaft, Verkehr, Handel und Verwaltung. ${ }^{124}$

\subsubsection{Die konfuzianische Schule als Übergang der öffentlichen Schulen}

Die Erziehungskonzepte in der öffentlichen Schule stellten auch eine Herausforderung an die Vorstellung der konfuzianischen Erziehung dar. In der ersten Phase stand die taiwanesischen Gelehrten vor einem Spannungsverhältnis zwischen alten chinesischen

\footnotetext{
123 Vgl. E. Patricia Tsurumi 1977, S. 18 und Wan Chih-ting 1959, S. 42. Das Fach Japanische Sprache diente der äußerlichen, das Fach Persönlichkeitspflege der innerlichen Assimilation. Vgl. Li Yuen-hui 1983, S. 7 und Wang Chin- chueh 2005, S. 144-183.

124 Vgl. Wu Wen-Hsing 1978, 264-291 und Hsieh Li-fa 1997, S. 100-102.
} 
Tradition und neuen japanischen Kultur. Weil die Mehrheit der taiwanesischen Gelehrten noch starke Verbidnung mit der chinesischen Tradition und Kultur hatten. Sie erwarteten immer noch, dass sie eine Amtstelle durch das Studium der konfuzianischen Lektüren und den Bestehen bei den kaiserlichen Beamtenprüfungen erwerben zu dürfen. Sie genossen ihr literarisches Leben durch Dichten und Aufsätzenschreiben weiter und betrieben weiter die erzieherischen Aktivitäten in den konfuzianischen Schulen. Zudem standen sie skeptische Abneigung die japanische Kultur und dem Unterrichtskonzept der japanischen öffentlichen Schule gegenüber, vor allem den Fächern Singen und Sport, die in der chinesischen Tradition wenig geschätzt wurden. Sie nannten die japanische öffentliche Schule als „barbarischen Schule“125 und schickten ihre Kinder wie zuvor in konfuzianische Schule. Nur die Kinder der Kaufleute sowie der mittleren und unteren Schichten besuchten die öffentliche Schule. ${ }^{126}$

Laut dem Prinzip des „Gradualismus“ hatte die Kolonialregierung die konfuzianischen Schulen nicht sofort abgeschafft, sondern „reformierte“ sie zu „Übergangsschulen“. Im Zuge dessen musste mindestens zwei Stunden pro Tag zusätzlich Unterricht in japanischer Sprache und Arithmetik, den vom Gouvernement anerkannten Lehrstoffen für öffentliche Schulen, erteilt werden. Darüber hinaus sollten die Lehrer auf die hygienischen Gewohnheiten der Schüler achten, denn es gab damals sehr viele Seuchen im Zusammenhang mit einer Mäuseplage, auch Malaria und Cholera traten auf, was die japanische Regierung durch schulische Erziehung zu bekämpfen versuchte. ${ }^{127}$

\footnotetext{
125 Weil die meisten chinesischen bzw. taiwanesischen Gelehrten China als Kulturzentrum der Welt vorgestellt haben und die anderen Teile der Welt als barbarische Gesellschaft angesehen haben. Vgl. 5.5.1.1. in dieser Arbeit.

${ }^{126}$ Vgl. ebd., S. 34-40, und Li Yuen-huei 1983, S. $91 f$.

127 Vgl. Hsu Pei-hsien 2001, S. 96-100 und Hsu Hsi-ching 2008, S. 408-423.
} 


\subsubsection{Erweiterung der elementaren Schulen}

Jedoch spielte die Haltung der taiwanesischen Gelehrten eine entscheidende Rolle für die Förderung der öffentlichen Schulen. Denn die japanische Regierung noch keine ausreichenden Mittel zur Erweiterung der öffentlichen Schulen zur Verfügung gestellt und nur die Gehälter der Lehrer übernommen hatte, während die anderen Ausgaben von den Gemeinden und reichen Gelehrten und Großgrundbesitzern getragen wurden. ${ }^{128}$ Mit der Einführung des Polizeisystems nahmen die taiwanesischen Gelehrten immer weniger an den Widerständen gegen die japanische Herrschaft teil. Mit dem Erlass des Schuledikts 1903/04 und der Aufhebung des kaiserlichen Beamtenprüfungssystems 1905 in chinesischem Kaiserreich erkannten die taiwanesischen Gelehrten, dass die Verbidung zwischen dem Studium der konfuzianischen Lektüren und dem Erwerb um die Amtstelle abgebrochen ist. Nachdem Japan den Krieg gegen den Russland 1905 gewonnen hatte, wurden viele Gelehrten in Ostasien daduch aufgeklärt, dass Japan durch die „Meiji Restauration“ zu neuer Weltmacht erfolgreich geführt wurde. D. H., dass eine Bewegung der Verwestlichung wie „Meiji Restauration“ einen „richtigen“ Weg für die künfige Entwicklung ihres Landes ist, z.B. „Hundertage Reform 1898“ wurde unter diesem Einfluss im Ching-Kaiserreich gefordert. Zugleich nahmen die taiwaneischen Gelehrten die Realität der japanischen Kolonialherrschaft über Taiwan wahr, dass die neue Weltmacht, Japan, in Taiwan für lange weilen wurde. Sie änderten dewegen ihre Haltung der japanischen öffentlichen Schule gegenüber. Sie schickten nicht nur ihre Kinder zur öffentlichen Schule, sondern auch forderten ihre Kinder die gleiche Bildungsqualität wie die japanischen Kinder erhalten zu können, und schickten sogar ihre Kinder direkt nach Japan zur Schule. ${ }^{129}$ Anhand der Statistik

\footnotetext{
128 Vgl. Hsu Pei-hsien 2001, S. 68.

129 Vgl. Hsu Hsi-ching 2008, S.397-427 und Li Kuan-chih 2006, S. 58-60.
} 
(Tabelle 1) zeigte es deutlich ab 1904 die Anzahl der Kinder in konfuzianischen Schulen jährlich abnahm, hingegen nahm die Anzahl der Kinder in den öffentlichen Schulen zu.

Mit dem „Edikt der Volksschule“ im März 1941 wurde die ethnische Unterteilung in den Schulen, einige Eingeborenenschulen ausgenommen, in Taiwan aufgehoben. Die Primar- und öffentlichen Schulen wurden $\mathrm{zu}$ „Volksschulen“ umgewandelt und sollten allen Schülern offen stehen, ungeachtet der ethnischen Zugehörigkeit. Allerdings wurden die Schüler in drei Stufen gemäß ihrer Kompetenz in der japanischen Sprache auf die ihrem Niveau entsprechende Volksschule verteilt. Kinder aus Japanisch sprechenden Familien erhielten Zugang zur Volksschule Nummer 1, in die auch japanische Kindern gingen. Die meisten taiwanesischen Kinder besuchten Nummer 2, die Volksschule für taiwanesische Kinder, während die Kinder der Ureinwohner wegen mangelnder japanischer Sprachkompetenz in die dritte Stufe eingeschult wurden. ${ }^{130}$

Im April 1943 wurde die sechsjährige Schulpflicht für Kinder im Alter zwischen acht und vierzehn Jahren eingeführt. ${ }^{131}$ Die Eintrittsquote in die Grundschule steigerte sich von 33,1\% im Jahr 1930 auf 41,5\% im Jahr 1935 und auf 71,31\% im Jahr 1943 (Tabelle 2). Damit war die relative Schulbesuchsrate in Taiwan die zweithöchste in Asien und wurde nur von Japan selbst übertroffen. ${ }^{132}$ Die schulische Landschaft wurde von konfuzianischer Schule zur modernen öffentlichen Schule umgewandelt.

\footnotetext{
130 Vgl. Wu Wen-hsing 2000, S.169.

131 Vgl. Li Yuen-hui 2005, S. 556-558.

132 Vgl. Chen Hsiao-chung 2005, S. 303-311.
} 


\subsubsection{Aufbau der Sekundarstufe}

Trotz anfänglichem Zögern wurde die höhere Bildung für taiwanesische Jugendliche aufgrund des Bedarfs an Lehrkräften in den öffentlichen Schulen durch die Einführung der elementaren Bildung sowie an Arbeitskräften für die Industrialisierung eingeführt und Bildungseinrichtungen für die Sekundarstufe II sowie die Tertiärstufe systematisch ausgebaut

1896 wurde die „Landessprachschule“ in drei Bildungsgängen eingerichtet: zur Ausbildung von Lehrkräften in den „öffentlichen Schulen“, zur höheren allgemeinen Bildung sowie zur höheren beruflichen Ausbildung für die mittleren Arbeitskräfte in den Bereichen Eisenbahn und Telegraphie (errichtet 1900), Agrarwirtschaft, Industriewirtschaft, Forstwirtschaft und Zuckerindustrie (errichtet 1904). ${ }^{133}$

1899 wurden drei dreijährige Lehrerausbildungsanstalten für öffentliche Schulen in Taipei, Taichung und Tainan - also im Norden, in der Mitte und im Süden Taiwans für jeweils 150 Studenten eingerichtet. ${ }^{134}$ Diese Qualifikation gewann jedoch keine gesellschaftliche Anerkennung. Bildungshungrige Jugendliche mussten nach Japan fahren, um dort die höheren Bildungsmöglichkeiten zu nutzen. ${ }^{135}$ Und immer mehr taiwanesische Jugendliche aus wohlhabenden Familien gingen nach China, Japan und Europa sowie in die USA, um eine weiterführende Bildung zu erhalten. ${ }^{136}$ Dort lernten sie die Zeitströmungen kennen, etwa die „Selbstbestimmung der Völker“ und Demokratie von Woodrow Wilson in den USA, ${ }^{137}$ Bewegungen zur

\footnotetext{
133 Vgl. Wu Wen-hsing 2000, S. 18.

134 Vgl. E. Patricia Tsurumi 1977, S. 16-23.

135 Vgl. Wang Chin-chueh 2002, S. 142 und E. Patricia Tsurumi 1977, S. $126 f$.

136 Vgl. Wu Wen-hsing 1992, S. 118-125.

137 1918, noch während des Ersten Weltkriegs, stellte US-Präsident Woodrow Wilson (1856-1924) das 14-Punkte-Programm“ (Fourteen Points) vor, das die Leitsätze von Demokratie, „Selbstbestimmung der Völker", kollektiver Sicherheit und freiem Handel als Basis einer neuen und friedlichen Ordnung der Weltpolitik sah. Vgl. Jürgen Osterhammel und Niels P. Petersson 2003, S. 77.
} 
„Selbstbestimmung der Völker“ wie die „Kulturbewegung des Vierten Mai 1919“ in China oder die „Unabhängigkeitsbewegung des 1. März 1919“ in Korea, welche sich gegen die japanische Kolonialmacht richtete. Als sie in ihr Land zurückkehrten, versuchten sie die Weltanschauung auch auf die taiwanesische Gesellschaft zu übertragen. Der Sohn des Großgrundbesitzers in Tai Chung, Ling Hsien-tang (18811956) ${ }^{138}$ und seine Anhänger, die meistens in Tokio studiert hatten, riefen 1914 die „Bewegung für die Gleichberechtigung zwischen Japanern und Taiwanesen sowie zur Selbstbestimmung der Taiwanesen“ ins Leben. Ling artikulierte die Wünsche nach demokratischen Freiheitsprinzipien und lokaler Autonomie, daher forderte er für japanische und taiwanesische Jugendliche die gleichen Rechte in Bezug auf politische Teilnahme, Pressefreiheit und Bildung. Immer mehr japanische Gelehrte und Politiker kritisierten fortan die „Separationsmaßnahmen“ des japanischen Gouvernements. Die Schuledikte von 1919 und 1922, unterstützt durch den ersten nicht adligen Premierminister Japans (1918-1921), Hara Takashi, gehen auf diese Strömungen zurück.

Nachdem 1907 die erste Mittelschule für japanische Jungen und 1910 für japanische Mädchen in Taipei errichtet worden war, verzögerte das Gouvernement indes den Aufbau der höheren allgemeinen Bildung für taiwanesische Jugendliche. 1912 forderte eine Initiative aus dem Kreis der Gelehrten und lokalen Grundbesitzer in Tai Chung die Errichtung einer Mittelschule für taiwanesische Jugendliche. Um die politische Stabilität zu wahren, kam das Gouvernement im Jahr 1913 den

\footnotetext{
${ }^{138}$ Als Ling Hsien-tang in Japan studierte, hatte er engen Kontakt mit dem führenden Reformer, Liang Chi-chao. Durch die dessen Ermutigung leitete Ling später die „Bewegung für Gleichberechtigung und Selbstbestimmung der Taiwanesen" ein und forderte die Einführung des parlamentarischen Systems. Er gründete den „Verein der Kultur Taiwans“ (1921) und die Volkspartei Taiwan (1927). Nach dem Zweiten Weltkrieg wurde er ins erste Parlament Taiwans gewählt. Yen Jung-chung 1971, S.13.
} 
Forderungen nach und errichtete 1915 die erste Mittelschule für Jungen mithilfe von lokalen Sponsoren in Taichung. ${ }^{139}$

Mit dem „Schuledikt für Taiwan aus dem Jahre 1919“ wurde das Bildungssystem dem in Japan angeglichen. In der sekundären Stufe wurde eine vierjährige Mittelschule für Jungen, eine dreijährige Mittelschule für Mädchen, eine fünfjährige Lehrerausbildungsstätte und eine dreijährige Berufsschule für Gewerbe, Handel und Agrarwirtschaft errichtet; weitere kurzzeitige bis zweijährige Ausbildungseinrichtungen kamen hinzu. In der Tertiärstufe sollten eine dreijährige Fachschule für die Ausbildung von Facharbeitskräften der Fachrichtungen Industrie, Handel sowie Agrar- und Forstwirtschaft, eine vierjährige Medizinfachschule sowie eine Universität folgen. ${ }^{140}$ Zudem wurden Lehrgänge in den Bereichen Eisenbahn, Telegraphie, Agrarwirtschaft, Industrie, Forstwirtschaft und Zuckerindustrie ausgestaltet. ${ }^{141} \mathrm{Im}$ Zuge der auf Südostasien gerichteten japanischen Politik und Industrialisierung wurden die dreijährigen Berufsschulen und „Ausbildungsstätten für kurzzeitige Förderkurse" rasch erweitert. ${ }^{142}$

Mit dem „revidierten Schuledikt für Taiwan aus dem Jahr 1919“, im Jahre 1922 vom achten Generalgouverneur (1919-1923), Den Kenjiro (1855-1930), erlassen, sollten die Unterschiede bei den Bildungsmöglichkeiten zwischen Japanern und Taiwanesen aufgehoben und die Angebote für taiwanesische Jugendliche erweitert werden. ${ }^{143}$ Doch tatsächlich ließ man durch die Barriere der Aufnahmeprüfung, die Kenntnisse der japanischen Sprache verlangte, nur einen kleinen Teil zur höheren Bildung zu,

\footnotetext{
139 Vgl. Chu Pei-chi 2000, S. 16-21.

140 Vgl. Wu Wen-hsing 1992, S. 100.

141 Vgl. Hsu Nan-hao 1984, S. 105-107.

142 Vgl. Wu Wen-hsing 2000, S. 18.

143 Vgl. ders. 2003, S. 527-547.
} 
wobei die meisten auf den Zweig der beruflichen Ausbildung gelenkt wurden. ${ }^{144}$ Die Japaner wollten auf ihre Privilegien in der Erziehung nicht verzichten, und nur wenige japanische Eltern schickten ihre Kinder auf die Schule für taiwanesische Kinder. ${ }^{145}$

Am Ende der japanischen Besatzungszeit gab es 22 Mittelschulen für taiwanesische Jungen, die 50,56\% der gesamten Schüler ausmachten, und 23 für taiwanesische Mädchen, das waren 36,46\% aller Schüler. Hinzu kamen insgesamt drei Lehrerausbildungsanstalten mit 347 taiwanesischen Schülern, das waren 13,13\% aller Schüler. In 27 Berufsschulen (neun agrar- und forstwirtschaftliche Schulen, neun gewerbliche Schulen, sieben Handelsschulen und zwei Schulen für Fischerei und Meereswesen) wurden 7876 taiwanesische Schüler (60,39\%) unterrichtet, und 90 „Ausbildungsstätten für kurzzeitige Förderkurse“ hatten 5531 taiwanesische Schüler $(71,66 \%)$ aufgenommen (Tabelle 2 und 3).

\subsubsection{Aufbau der Tertiärstufe}

1919 wurde eine Medizinschule - 1899 für taiwanesische Jugendliche errichtet - zur Medizinfachschule und zum „Forschungszentrum Krankheiten und Infektionen in tropischen Gebieten“ ausgebaut und 1936 der Japanischen Reichsuniversität in Taipei angegliedert. Zudem wurden ab 1919 dreijährige ordentliche Fachschulen für Landund Forstwirtschaft (1919 in Taipei), Handelswesen (1919 in Taipei) und Industriewesen (1927 in Tainan) sowie zwei höhere allgemeinbildende Schulen zur Vorbereitung auf ein Universitätsstudium, die dreijährige höhere allgemeinbildende Schule in Taipei (1922) und das dreijährige Studienkolleg an der Universität Taiwan

\footnotetext{
144 Vgl. Yanaihara Tadao 1987, S. $146 f$.

145 Vgl. ebd., S. 144.
} 
(1941), errichtet. ${ }^{146}$ Im Jahr 1928 folgte die Japanische Reichsuniversität in Taipei (Taihoku Imperial University), ein Forschungszentrum für Kultur, Wirtschaft, Politik, Pflanzen, Klima sowie Naturschätze in Südostchina und Südostasien. Die Zwecke für Errichutng der Universität waren: erstens Fachleute und Führungskräfte für die koloniale Verwaltung heranzubilden, zweitens den Wunsch der taiwanesischen Jugendlichen nach höherer Bildung zu erfüllen und die Tendenz zum Auslandsstudium und damit antijapanische Strömungen auszubremsen. ${ }^{147}$ Drittens sollte die Universität der auf Südostasien ausgerichteten Politik dienen. ${ }^{148}$ Die universitäre Bildung blieb jedoch den Japanern vorbehalten, denn die meisten taiwanesischen Schüler scheiterten an der strengen Ausleseprüfung in japanischer Sprache, außerdem blieb die einmal festgelegte Quote für japanische Schüler unangetastet. Bis zum Ende der Besatzungszeit betrug der Anteil der taiwanesischen Studenten an der Universität nur 15,91\%, an der Fachhochschule 17,15\% und im Studienkolleg 11,00 \% (Tabelle 3). Das Verhältnis zwischen taiwanesischen und japanischen Studenten betrug in der Fakultät für Literatur- und Politikwissenschaft $45 \mathrm{zu}$ 277, in der Fakultät für Technik und Agrarwissenschaft $37 \mathrm{zu}$ 303, allein in der Fakultät für Medizin 79 zu $97 .{ }^{149}$

\footnotetext{
146 Vgl. Lai An-ming 1996, S. 141f.

147 Vgl. Wu Mi-cha 1991, S. 169f.

148 Vgl. Chiang Pei-ching 1997, S. 38.

149 Vgl. Lin Chian-fu 1996, S. 168.
} 


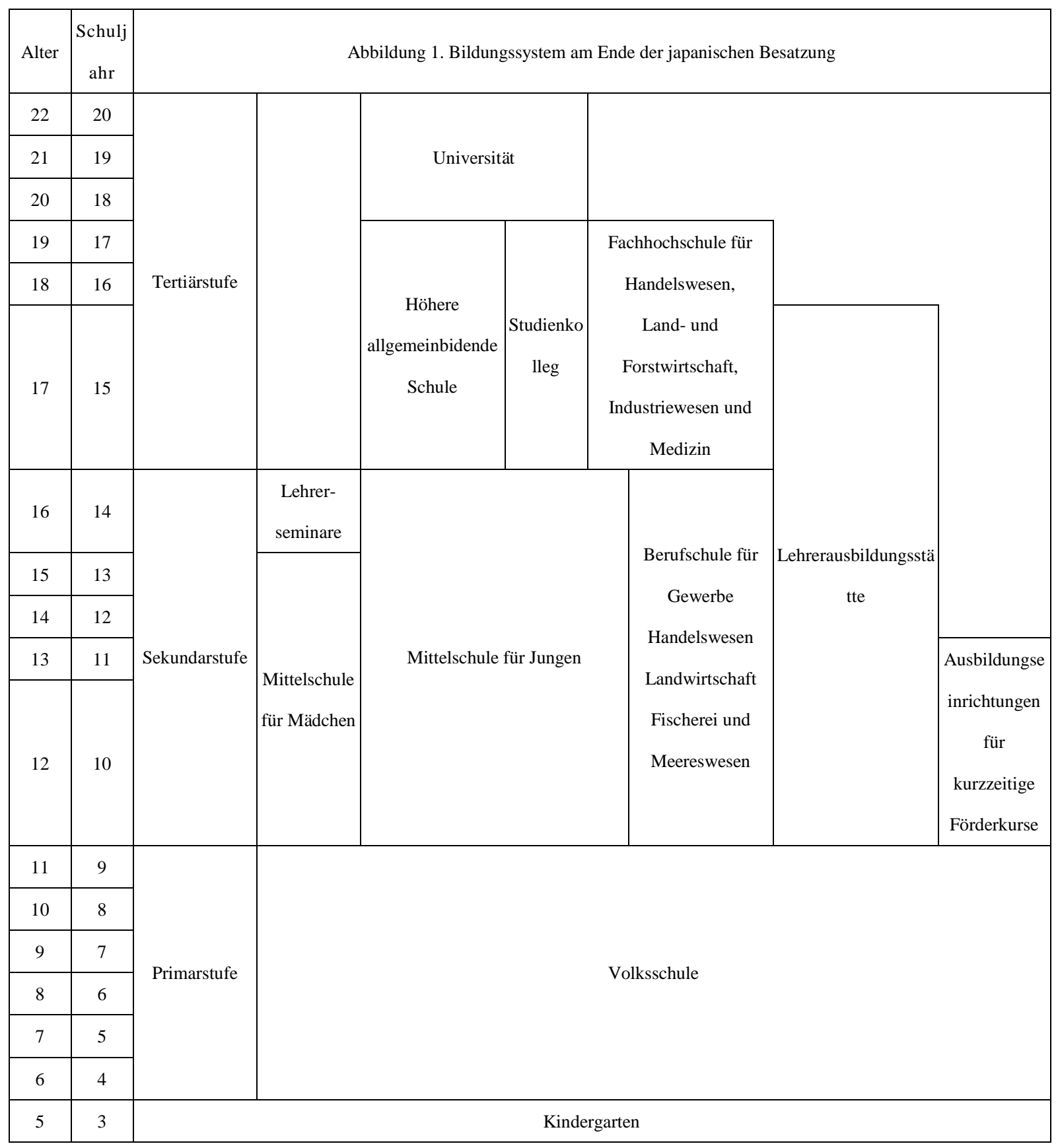


5. Debatte über die Gestaltung eines modernen staatlichen Bildungswesens 1898 bis 1949

Nachdem das KMT-Regime auf Taiwan die Macht 1945 übernommen hatte, übertrug es sein Aufbauprogramm eines Nationalstaates auf die Erziehung. Die seit 1898 in China geführte Debatte über die Gestaltung eines modernen staatlichen Bildungswesens und die wurde weitergeführt und Letztere schließlich als leitendes Konzept beim Aufbau des taiwanesischen Bildungswesens gemäß den „Drei Prinzipien des Volkes“ des KMT-Regime praktiziert. In diesem Abschnitt wird ein Rückblick auf die Debatte zu Modernisierung des Bildungswesens auf dem chinesischen Festland vorgestellt.

\subsection{Entwurf eines modernen staatlichen Bildungswesens im Zug der Hundert- tage Reform 1898}

Den Absolventen der modernen Fachschulen sowie den Auslandsstudenten innerhalb der „Selbstärkungsbewegung““150 blieb die Anerkennung jedoch versagt, da die westlichen Kenntnisse nach wie vor geringgeschätzt und das Lernen der westlichen Kultur als Schande und Selbsterniedrigung empfunden wurde. In einigen Fällen demonstrierten chinesische Gelehrte sogar offen ihre feindliche Haltung gegenüber der okzidentalen Kultur. ${ }^{151}$ Dennoch wurden zahlreiche westliche Werke ins Chinesische übersetzt, z.B. von Yen Fu, ${ }^{152}$ und alsbald von vielen Gelehrten gelesen, so etwa die neuen Soziallehren wie „Evolution and Ethics“ (1896), „Darwinsche Evolution“ und „Sozialdarwinismus“. Bevorzugte Autoren waren Charles Darwin,

\footnotetext{
${ }^{150} \mathrm{Vgl}$. 3.3.1. in dieser Arbeit.

151 Vgl. Li Hsi-suo 1987, S. 60- 63.

152 Yen Fu (1854-1921) besuchte von 1867-1871 die Schiffbau- und Schifffahrtschule in Fuzhou und studierte von 1877-1879 am Royal Naval College in Großbritannien. Er war der erste Rektor der Universität Peking und übersetzte wichtige Werke der britischen Denker ins Chinesische, um dadurch die chinesischen Gelehrten zur Reform zu bewegen. Vgl. Wu Chan-liang 1999, S. 103-176.
} 
Herbert Spencer und Thomas Henry Huxley. ${ }^{153}$ Die Weltanschauung der westlichen Kultur und die These des „Sozialen Darwinismus“ hatten Gelehrte wie Kang You-wei ${ }^{154}$ und Liang Chi-chao ${ }^{155}$ zur Gestaltung einer radikalen Reform des politischen Systems und der Gesellschaft angespornt, jedoch lehnten sich deren Forderungen stark an in Japan an. Nicht nur wegen der kulturellen Ähnlichkeit und der Nachbarschaft, sondern auch wegen der erfolgreichen Erfahrung der Meiji-Restauration, durch die Japan in kurzer Zeit Weltmachtstatus erlangt hatte, waren die Reformer große Bewunderer Japans.

Nach dem verlorenen Krieg gegen Japan 1895 plante Kaiser Kuang-hsu (1871-1908), beraten von Kang You-wei und Liang Chi-chao u. a., eine Reform in Bezug auf politische, wirtschaftliche, militärische, kulturelle und erzieherische Belange, womit ein Grundriss zur Modernisierung Chinas gezeichnet wurde. Zur Bildungsreform wurden folgende Maßnahmen vorgeschlagen: Auf allen Ebenen der kaiserlichen Beamtenprüfungen sollte das herkömmliche ,achtgliedrige“ Aufsatzschreiben durch pragmatische Formen ersetzt werden, pragmatisches Wissen statt literarischer Schreibkunst sollte das Kriterium für die Auswahl der Kandidaten sein und Wirtschaftsfragen als Themen in der Prüfung eingefügt werden.

Die Gründung der Peking Metropol-Universität sollte einen offiziellen Verlag und ein

\footnotetext{
153 Vgl. Wu Chan-liang 1999, S. 103-176 und Kuo Cheng-chao 1972, S. 566-570.

154 Der Gelehrte und Reformer Kang You-wei (1858-1927) begann sich nach Studien der Darwin'schen Evolution ab den 1890er-Jahren mit einer Reform des chinesischen Kaiserreichs zu beschäftigen. Im Gegensatz zu Sun Yat-sen sah er die Zukunft Chinas nicht in einer Abschaffung der Monarchie zugunsten einer Republik. Deswegen bemühte er sich nach deren Gründung um den Wiederaufbau der Monarchie. Vgl. Pan Tai-hsiung 1995.

${ }^{155}$ Liang Chi-chao (1873-1929), ebenfalls ein bedeutender Reformer, zudem bekannter Historiker und Journalist, hatte einen traditionellen Bildungsweg beschritten und vorzügliche Ergebnisse bei den kaiserlichen Beamtenprüfungen abgelegt. Als Schüler von Kang You-wei teilte er dessen Interesse, China zu reformieren. Er bereiste viele Länder und schrieb zahlreiche Reiseberichte. Später veröffentlichte er unterschiedliche Formen von Zeitungen und kämpfte um die Pressefreiheit in China. Vgl. Pan Tai-hsiung 1995.
} 
Übersetzungsbüro mit einschließen. Alle althergebrachten Bildungsanstalten sollten in Schulen mit chinesischen und westlichen Disziplinen umgewandelt werden. Die Bildungsanstalten auf Provinzhauptstadtebene sollten Universitäten, diejenigen auf Bezirks- und Stadtebene Mittelschulen und die auf Unterbezirks- und Kreisstadtebene Grundschulen werden. Alle privaten Bildungsstätten sollten zu modernen Schulen umfunktioniert werden, auch die Fachschulen für Eisenbahnbau und Bergbau.

Am Zeitgeschehen interessierte Adlige, die sich weiterbilden wollten, konnten ins Ausland geschickt werden. Besonders begabte Studenten aus den verschiedenen Provinzen sollten zum Studium nach Japan entsandt werden. ${ }^{156}$ Die an Japan orientierten Reformer wurden durch die japanische Regierung unterstützt, damit japanische Kultur und Politik in China Einfluss gewinnen konnten. Japans Ambitionen lagen darin begründet, die führende Rolle bei der chinesischen Modernisierung vor den anderen Weltmächten wie Großbritannien, Frankreich, Preußen, Russland und den USA zu übernehmen. ${ }^{157}$ Seit 1898 besichtigten zahlreiche chinesische Beamte und mandschurische Adlige Japan. Tausende von ausgesuchten Studenten fuhren nach Japan zum Studium der japanischen Erfahrungen bei der Modernisierung. ${ }^{158}$

Weil sich die Konservativen gegen die Reform stellten, wurde sie bereits hundert Tage nach Ankündigung des Dekrets abgebrochen - diese Refom wurde deswegen als Hunderttage-Reform genannt-, jedoch ihre Forderungen wirkten fort.

\footnotetext{
156 Vgl. Verlag für fremdsprachige Literatur, Peking 1978, S. 97-99.

157 Vgl. Chien Man-chien und Chin Lin-hsiang 1996, S. 62.

158 Der japanische Einfluss wurde zudem durch zahlreiche chinesische Studenten in Japan, durch die Aufnahme chinesischer politischer Flüchtlinge und nicht zuletzt durch das erhöhte Ansehen Japans nach seinem Sieg über Russland 1905 verstärkt. Im Jahr 1896 wurden nur 13 Schüler nach Japan entsandt, 1903 war die Zahl bereits auf 1300 gestiegen, im Jahr 1906 wird die Zahl auf ca. 12.000 geschätzt. Vgl. Li Hsi-suo 1987, S. 127; Chien Man-chien und Chin Lin-hsiang 1996, S. 58-60 sowie Huang Fu-ching 1975, S. 147-187.
} 


\subsection{Das erste Edikt zur Gestaltung eines modernen staatlichen Bildungswesens im Jahr 1903/04}

Nach dem Scheitern des Boxeraufstands $1900{ }^{159}$ waren die Konservativen gezwungen, radikale Reformen des Bildungssystems durchzuführen. Zwar erkannten die chinesischen Beamten und Gelehrten die Notwendigkeit einer Anpassung an das westliche Bildungssystem, aber die Kulturkonservativen hofften immer noch, das konfuzianische Gedankengut als Grundlage retten zu können. Chang Chih-tung, ${ }^{160}$ der mit der Gestaltung des modernen staatlichen Bildungswesens beauftragt war, propagierte in seinem Aufsatz „Aufforderung zum Lernen“ folgendes Konzept: „die chinesische Weltanschauung als Grundlage und das westliche Wissen zum praktischen Gebrauch“. ${ }^{161}$ Chang übte Kritik an der christlichen Erziehung und den Reformprogrammen von 1898. Kultur werde dort nicht als Einheit betrachtet, sondern als Ansammlung voneinander unabhängiger materieller, institutioneller und geistiger Dimensionen. Die materielle und institutionelle Dimension sollte nun in den Dienst der Modernisierung und Industrialisierung Chinas gestellt werden, nicht aber die geistige. Das hieß, die westlichen Kenntnisse wie Technik und angewandte Naturwissenschaften sollten zur Entwicklung von Industrie und Handel übernommen, die konfuzianischen Werte und Normen aber daneben beibehalten werden. ${ }^{162}$

\footnotetext{
159 Im Jahr 1900 verübte die Geheimgesellschaft der Boxer Attacken gegen Ausländer in China und chinesische Christen, anschließend entbrannte ein Krieg zwischen dem chinesischen Kaiserreich und den vereinigten acht Mächten (bestehend aus dem Deutschen Reich, Frankreich, Großbritannien, Italien, Japan, Österreich, Ungarn, Russland und den USA). Mit der Niederlage der Chinesen wurde ein so genanntes „Boxerprotokoll“ im September 1901 abgeschlossen. Vgl. Joseph W. Esherick 1987.

${ }_{160}$ Chang Chih-tung (1837-1909) war ein vom Konfuzianismus sehr stark geprägter Beamter der Ching-Dynastie. Durch Einfluss von Missionaren wie Timothy Richard setzte er sich seit 1882 mit westlichen Kenntnissen auseinander. Als Gouverneur in Hunan und Hupei von 1889 bis 1907 beschäftigte er sich mit der Erziehungsreform der konfuzianischen Schulen und der Errichtung moderner Schulen wie Militärschulen, Schulen für Agrarwirtschaft, Industrie und Handel sowie Schulen für die Lehrerausbildung und war für die Verbesserung des ursprünglichen Entwurfs zur Gestaltung des modernen Schulsystems im Jahre 1902 zuständig. Vgl. Su Yun-fun 2005, S.125-135.

${ }^{161}$ Vgl. Chen Fang-ping und Bi Chen 1996, S. 349f.

162 Vgl. Wang Er-min 1969, S. 55-66.
} 
Im Jahr 1902 wurde auf Befehl der Kaiserwitwe Tzu-hsi der erste „Entwurf zur Gestaltung eines modernen Bildungswesens“ von Chang Chih-tung, Chang Pai-hsi (1847-1907) ${ }^{163}$ und Jung Ching (1859-1917) nach dem oben genannten Prinzip und auf Basis der Reformpläne 1898 erstellt ${ }^{164}$ und 1903/04 revidiert und bekannt gegeben. Zielsetzung war es, „sowohl die Loyalität gegenüber der Monarchie zu stärken, die Verehrung von Konfuzius und die Beachtung der öffentlichen Sitte wieder mehr in den Mittelpunkt zu rücken als auch eine Förderung der sportlichen und militärischen Übung zu forcieren. “165 Auch sollte die Reform zum Studium der Kenntnisse für die praktische Brauchbarkeit anregen. Der Entwickler des Edikts, Chang Pai-hsi, wies darauf hin, ,gleich auf welcher Stufe und in welcher Art von Schule, Loyalität und Gehorsamkeit sollten als Ziel der Erziehung durch das Bildungswesen eingehalten werden und das Studium der chinesischen Klassik, Geschichte und Geographie als Grundlage der Erziehung erhalten bleiben, um die Reinheit des Herzens der Schüler zu bilden. Erst dann können die Schüler ihre Kenntnisse und Fähigkeiten durch die Aneignung von westlicher Kultur und Technik erweitern.“166 Das Ziel war nicht allein die Ausbildung künftiger Beamter, sondern auch die Ausbildung von Fachleuten in unterschiedlichen Bereichen wie Militärwesen, Landwirtschaft, Industrie, Handel und Erziehungswesen, wobei die Loyalität gegenüber Monarchie und konfuzianischer Tugend gestärkt werden sollte.

Nach dem Erlass des Schuledikts 1903/04 wurden einerseits konfuzianische Schulen in moderne Schulen umgewandelt, andererseits zahlreiche moderne Bildungs-

163 Chang Pai-hsi (1847-1907) hatte den Titel Chin-shih erlangen und war Mitglieder von Hanlin Akademie. Er nahm an der politischen, wirtschaftlichen und erzieherischen Reform am Ende des Ching-Kaiserreichs teil. Er hatte 1898 die Errichtung der ersten kaiserlichen Universität Peking gestaltet, anschließend entwarf er die Edikte zur Gestaltung des modernen Bildungswesens Chinas. Vgl. Su Yun-fun 2005, S.119-125.

164 Vgl. Liou Wei-chih 2006, S. 30-57; Chien Man-chien und Chin Lin-hsiang 1996, S. 93123.

165 Vgl. Chang Chih-tung 1971, S. 209.

166 Vgl. ebd. S. 209. 
einrichtungen neu errichtet, so 1905 das Bildungsministerium als zentrale Verwaltung, um die Modernisierung des Bildungswesens durchzuführen. Weitere Bildungsbehörden waren für die Lehrinhalte der verschiedenen Stufen (primäre, mittlere und höhere Bildung) und Bildungsbereiche (allgemeine Bildung, berufliche Ausbildung und Lehrerausbildung) zuständig. ${ }^{167}$ Außerdem durften nur Beamte bzw. Gelehrte, die sich mit der Administration des japanischen Bildungswesens beschäftigt hatten, in den Bildungsbehörden eingestellt werden. ${ }^{168}$

Das Schuledikt von 1903/04 gilt in der chinesischen Erziehungsgeschichte als Übergangspunkt vom traditionellen zum modernen Erziehungssystem. Die wesentliche Funktion der Erziehung war nicht mehr allein die Ausbildung der Beamten und Führungspersonen, sondern auch die Qualifikation zu beruflicher Tätigkeit in unterschiedlichen Bereichen von Gesellschaft und Produktion.

\subsection{Das Edikt für Mädchenerziehung 1907}

In China waren Schule und die Ausbildung zum Beamten den männlichen Bürgern vorbehalten, während die traditionellen Erziehungsinhalte für die Frau an den in der Han-Dynastie von Pan Chao (49-120) verfassten Aufsätzen „Gebote der Frauen“ ausgerichtet waren. Darin beschreibt die Autorin die „drei Gehorsamspflichten und vier Tugenden“ der Frau in den drei Lebensphasen als Tochter, Ehefrau sowie Schwiegertochter und Mutter, z.B. Treue, Persönlichkeitspflege, angemessenes Reden und Benehmen, geputztes Äußere sowie Beherrschung der Haushaltstechnik. ${ }^{169}$ Die Idee einer modernen Mädchenerziehung wurde erst durch die christliche

\footnotetext{
167 Vgl. Lin Ben 1977, S. 1-4.

168 Vgl. Liou Te-mei 1989, S. 303f.

169 Vgl. Bettine Birge 1989, S. 325-367; Michael Freudenberg 1985, S. 12-16. Zur Rolle der Frauen unter dem kaiserlichen Beamtenprüfungssystem siehe Harriet T. Zurndorfer 2006, S. 207-224.
} 
Mädchenschule in China bekannt. ${ }^{170}$ Der Reformer Liang Chi-chao stellte in seinem Aufsatz „Über die Frauenerziehung“ (1897) - unter Einfluss der Lehre Darwins - fest, dass die Schwächen eines Landes im fehlenden Lernen der Frauen begründet lägen. ${ }^{171}$ Wenn man seine Kultur bewahren wolle, sollte man auf die Ausbildung der Mütter Wert legen. ${ }^{172}$ Daher forderte er sowohl die Abschaffung des Füßebindens ${ }^{173}$ als auch die Erziehung der Frauen zur Selbständigkeit und die Gleichberechtigung der Mädchen in der Bildung. ${ }^{174}$ Unter dem Einfluss dieser Ideen wurden zahlreiche Mädchenschulen von aufgeklärten Gelehrten nach der „Hunderttage Reform 1898“ gefordert und gefördert. ${ }^{175}$ Die Mädchenerziehung - im Schuledikt von 1903/04 noch nicht erwähnt - wurde im Schuledikt von 1907 mit einbezogen. ${ }^{176}$

\subsection{Bildungsreformen zum Beginn der Grüdnung der Republik China}

China wurde Beginn des 20. Jahrhunderts zum „Rummelplatz für abendländliche Ideen“, wie Liu Hui-ru (1993) schrieb: „Was Europa in einem langen geistigen Prozess an Denken hervorgebracht hatte, wurde durcheinandergeworfen, um dann nach dem Prinzip der Beliebigkeit das eine oder andere herauszupicken und zur Norm $\mathrm{zu}$ erheben. Alles wurde auf eine einzige pseudo-synchrone Zeitebene projiziert: Platon neben Plechanow, Napoleon und Nietzsche, Dewey neben Dostojewski, Russell neben Rousseau - alles stand dicht gedrängt in einer Regalreihe.“177 Die Intellektuellen versuchten, aus den verschiedenen Strömungen wie Liberalismus,

\footnotetext{
170 Vgl. Huang Chi-wen 2005, S. 27-36.

171 Vgl. Bai Limin 2005, S. 184-187.

172 Vgl. Liang Chi-chao 1960, S. 34-37.

173 Entsprechend der neokonfuzianischen Vorstellung, wurde das Binden der Füße mit der Bindung der Frauen an Haus und Familie gleichgesetzt, denn dadurch waren Aktivitäten sehr eingeschränkt. Die Abschaffung bedeutete eine Befreiung von der traditionellen Rolle der Frau. Vgl. Michael Freudenberg 1985, S. 17-19.

${ }^{174}$ Vgl. Tu Hsueh-yuen 1997, S. 89-91.

175 Vgl. Michael Freudenberg 1985, S. 158-197.

176 Vgl. Huang Chi-wen 2005, S. 91-102.

177 Zit. nach Liu Hui-ru 1993, S. 50.
} 
Anarchismus, Sozialismus, Pragmatismus, Positivismus, Materialismus etc. die weitere Entwicklung Chinas zu gestalten, so auch die Reformen des staatlichen Bildungswesens. Folgend schildern zwei entscheidene Bildungsreformen zum Beginn der Gründung der Republik China von 1912 bis 1927.

\subsubsection{Reform des staatlichen Bildungswesens nach der Gründung der Republik 1912}

Tsai Yuen-pei, ${ }^{178}$ der erste Bildungsminister, gilt als eine der Schlüsselfiguren nach Gründung der Republik China, die sich mit dieser Frage beschäftigten, wohin das Bildungswesen der neuen Republik führen sollte. Tsai war einer der Männer, die nach dem Russisch-Japanischen Krieg (1904-05) nach Preußen entsandt wurden, um die Erfahrung bei der Gestaltung des staatlichen Bildungswesens vor Ort kennenzulernen. ${ }^{179}$ Wilhelm von Humboldt war sein Vorbild, als er das Schuledikt von 1912/13 auf der Basis seiner Erfahrungen in Preußen entwarf, das auf der demokratischen und republikanischen Idee von Freiheit, Gleichheit und Brüderlichkeit basierte. Daher sollten feudale Elemente wie Amtstitel für die Schulabsolventen abgeschafft und konfuzianische Texte in den Grund- und Mittelschulen sowie der Lehrerausbildung nicht länger gelehrt werden. Zielsetzung der Bildung sei nicht mehr die Loyalität gegenüber der Monarchie die Verehrung von Konfuzius, sondern die Entfaltung des Individuums und des modernen Staates. ${ }^{180}$ Kernpunkt sei die staatsbürgerliche Moralerziehung, ergänzt durch pragmatische Erziehung, wie Sport und Militärerziehung, sowie die Vervollkommnung der

178 Bevor der Pädagoge Tsai Yuen-pei (1868-1940) das Amt des Bildungsministers in der Republik China übernahm, hatte er drei Jahre, 1908 bis 1911, in Leipzig studiert. Aus Protest gegen den neuen konservativen Präsidenten Yuen Shih-kai trat er vom Amt zurück. Obwohl seine Amtszeit nur gut sechs Monate dauerte, war es ihm gelungen, die Grundlage für das gesamte Bildungswesen zu legen. Sein Einfluss wirkte weiterhin, vor allem durch sein Amt als Rektor der Universität Peking (1916-1927). Vgl. Wang Pei-li 1996 und Cai Jian-guo 1998.

179 Vgl. Wang Lun-shen 2002, S. 22.

180 Vgl. Tsai Yuen-pei 1984, S. 130-137. 
Persönlichkeit durch ästhetische Erziehung. Den Mädchen wurde nun ebenfalls das Recht auf Bildung zuerkannt; und Koedukation in der Grundschule war ein Novum in der chinesischen Erziehungsgeschichte. Außerdem wurden die konfuzianischen Erziehungsideale im staatlichen Bildungswesen endgültig beseitigt und die naturwissenschaftliche Fächer sowie die Vermittlung praktischer Kenntnisse in Agrarwirtschaft, Handelswesen, Haushaltwesen, Zeichnen und Singen als wichtige Inhalte der schulischen Erziehung aufgenommen. ${ }^{181}$ Dies bildete die Basis für die antikonfuzianische „Bewegung der Kulturerneuerung“ ${ }^{182}$ welche sich in den 1910erund 1920er-Jahren herausbildete.

\subsubsection{Reform des Bildungswesens nach der „Bewegung des Vierten Mai 1919“}

Die Revolutionäre hatten das Kaiserreich 1911 zwar zum Sturz geführt, aber sie verfügten weiterhin weder über eigene Streitkräfte noch über politische Macht, um der Revolution in ganz China zum Sieg zu verhelfen, was schließlich zum Kompromiss mit konservativen Kräften führte. Zunächst wollte ein wichtiger Militär der Ching-Dynastie, Yuen Shih-kai (1859-1916), das Amt des Präsidenten durch parlamentarische Wahlen übernehmen und im Jahr 1915/16 sogar den Thron durch Gründung einer neuen Dynastie besteigen.

China war zu jener Zeit kein einheitlicher Staat mehr. Im Versailler Vertrag (1919) wurde festgelegt, dass die ursprünglich deutsche Kolonie Shangtung an Japan

\footnotetext{
181 Vgl. Tsai Yuen-pei 1984, S. 130-137.

182 Der Effekt der Bewegung des vierten Mai 1919 führte zu einer radikalen Abwendung von der chinesischen Tradition, die auf dem Konfuzianismus fußte und zugleich eine „Bewegung der Kulturerneuerung“" wie die sogenannte Bewegung für die umgangssprachliche Literatur (Pai-hua wen yun-tung), dies heißt: Sie versuchten die klassische Literatur von den Regeln der chinesischen Kunstsprache (Wen-yen), die man nur aufgrund eines langen Studiums und durch intensive Übung beherrschen kann, zu befreien und einen umgangssprachlichen Literaturstil (Pai-hau-wen) zu schaffen. Vgl. Chow Tes-tsung 1971.
} 
übergeben werden sollte, was Empörung hervorrief, insbesondere deshalb, weil das Regime in Peking gegen die „ungerechten Verträge“ nichts unternehmen konnte. Unter Einfluss des US-Liberalismus, vor allem Woodrow Wilsons Forderung nach einer „Selbstbestimmung der Völker“ von 1918, sowie der erfolgreichen Sowjetischen Revolution 1917 wurde der Kampf gegen die Weltmächte ausgerufen und die „Bewegung des Vierten Mai 1919“ ins Leben gerufen, um das Nationalbewusstsein der Bevölkerung zu stärken. Sie rief zu Antitradition, Antiimperialismus sowie zur Erneuerung von Kultur und Erziehung auf und protestierte gegen das schwache Regime in Peking. ${ }^{183}$ Der Effekt dieser Bewegung war eine radikale Abwendung vom Konfuzianismus, wohingegen nun die Werte und Lebensweisen der abendländischen Kultur als erstrebenswert galten. Vor diesem Hintergrund ist die Reform des Bildungswesens im Jahr 1922 zu sehen.

Da die schulische Entwicklung in den einzelnen Provinzen sehr unterschiedlich verlief, hatten Erzieher und Pädagogen kurz nach Einführung des Schuledikts von 1912/13 die einheitliche Regulierung kritisiert, der es an Flexibilität mangele und die keine Rücksicht auf lokalen Besonderheiten nehme. Sie forderten ein neues Schuledikt, zumal sich die Intellektuellen vom Einfluss Japans im Bildungswesen befreien und sich den Zeitströmungen angleichen wollten, genannt seien hier Militarisierung und nationalistische Ausrichtung sowie die Verstärkung polytechnischer und naturwissenschaftlicher Inhalte der Erziehung, welche für alle zugänglich sein sollte, und Reformpädagogik. ${ }^{184}$ Verschiedene Gesellschaften

\footnotetext{
${ }^{183}$ Vgl. Chow Tes-tsung 1989, S. 8-10; Wolfgang Franke 1957, S. 7-10 und 16-18; Jürgen Domes 1969, S. 38-44.

${ }^{184}$ Reformpädagogik setzte Ende des 19. Jahrhunderts und im ersten Drittel des 20. Jahrhunderts neue Impulse und rief zur Reform von schulischer Organisation, Didaktik und Erziehung gegen Lebensfremdheit und Autoritarismus der vorherrschenden „Paukschule“ auf. Sie war stark beeinflusst von der Jugendbewegung, der Frauenbewegung, der Arbeiterbewegung und der Kunsterziehungsbewegung. Einige Reformpädagogen verbanden ihre liberale Grundhaltung mit sozialem Engagement, daneben
} 
wurden gegründet, z.B. „die chinesische Gesellschaft für Naturwissenschaft“, „für Berufsausbildung“, „für die Entwicklung einer neuen Erziehung“ sowie „für Volkserziehung“ und die „National Federation of Educational Association“. Sie alle versuchten eine Änderung der chinesischen Erziehungslandschaft zu bewirken. ${ }^{185}$

Die Mitglieder der einflussreichen „National Federation of Educational Association“"waren Erzieher und Pädagogen aus allen Provinzen Chinas. Schon auf der ersten Jahreskonferenz im Jahre 1915 wurde der „Entwurf für ein neues Bildungswesen“ thematisiert. Dies stieß eine öffentliche Debatte an, auch „Bewegung für ein neues Bildungswesen“ genannt. ${ }^{186} 1919$ wurde aus ihren Reihen die „Chinesische Gesellschaft für die Entwicklung der neuen Erziehung“ sowie die „Zeitschrift für eine neue Erziehung“ gegründet, welche progressiven Erziehungsgedanken $\mathrm{zu}$ vermitteln suchte und für die neuen Prinzipien im Bildungsbereich kämpfte.

Auf Einladung der „National Federation of Educational Association“ besuchte John Dewey 1919 China. Er blieb 26 Monate, reiste im ganzen Land umher und hielt fünf größere Vorlesungen an der Universität Peking. Seine Reden erschienen als Buch, das Rekordauflagen erreichte. Seine Thesen sowie die anderer bedeutender US-amerikanischer Pädagogen wie Twiss, ${ }^{187}$ McCall $^{188}$ und Monroe verbreiteten sich im Land und wurden auch in das soeben erstellte Schuledikt aufgenommen. Paul Monroe, ${ }^{189}$

\footnotetext{
gab es auch völkische Vertreter. Vgl. Su Yun-feng 1981, S. 1-31.

185 Vgl. Chien Man-chien und Chin Lin-hsiang 1996, S. 228.

186 Vgl. ebd., S. 253.

187 Der Pädagoge George R. Twiss wurde 1922 im Rahmen einer Untersuchung für die Einführung der naturwissenschaftlichen Erziehung in der Mittelschule nach China eingeladen. 1926 veröffentlichte er „Science and Education in China“. Vgl. Liou Wei-chih 2006, S. 75-87.

188 W.A. McCall, Pädagoge der Universität Columbia, der dort über Messungsmethoden der Lernleistung forschte, besuchte ebenfalls 1922 das Land und stellte die Entwicklung standardisierter Bewertungstests sowie seine pädagogischen Ansätze vor. Vgl. ebd.

189 Monroe (1869-1947), Dekan des „Teachers College in the Columbia University“, hatte 1913 zum
} 
der sich mit der Gestaltung des chinesischen Schulsystems beschäftigte, hielt 1921 in Kuangtung eine Reihe von Vorträgen, er plädierte für die Abschaffung des Studienkollegs, die Verkürzung der Schulzeit und die Einführung eines Fächerwahlsystems mit Kreditpunkten. Seine Thesen übten auf die Gestaltung des Bildungswesens starken Einfluss aus. ${ }^{190}$ Nach siebenjährigen demokratischen Auseinandersetzungen über verschiedene Vorschläge und Reformentwürfe aus unterschiedlichen Provinzen und Gesellschaftsschichten wurde schließlich die Endfassung für die Reform im Jahr 1922 erstellt, bezeichnet als „Bildungswesen im Jahr 1922“ oder „,neues Bildungswesen“. ${ }^{191}$

Der Fokus des neuen Schuledikts 1922 lag auf der Persönlichkeitsentwicklung der Schüler, was durch Gesamtschulen mit unterschiedlichen Lehrgängen sowie durch die Verlängerung der Schulzeit in der Sekundarstufe erreicht werden sollte. ${ }^{192}$ Lokale Selbstbestimmung spiegelte den demokratischen Geist der Neuerungen wider. ${ }^{193}$ Diese liberalisierten und anti-konfuzianischen Erziehungseinstellungen des Schulgesetzes von 1922 gewannen dadurch ihre Bedeutung in der schulischen Erziehung sowie die lokalen Regierungen erhielten mehr Freiheiten, über die Gestaltung der Schule zu bestimmen, was jedoch ein sehr differenziertes und uneinheitliches Erziehungswesen verursachte. Nach der Machtübernahme der KMT

ersten Mal China besucht, dort unterrichtete er 1921 und beschäftigte sich mit der Erziehungsentwicklung. Im Jahr 1922 veröffentlichte er seine Untersuchungsergebnisse über chinesische Erziehung mit dem Titel: „A Report on Education in China: For American Educational Authorities“ in New York. Vgl. auch He Ching-chin 1985 und Yang Tsui-hua 1991. Zur Debatte über die Gestaltung des neuen Bildungswesens unter den chinesischen Gelehrten und die Besonderheit des US-amerikanischen Schulsystems siehe Wang Lun-shen 2002, S. 48-86.

190 Vgl. Chien Man-chien und Chin Lin-hsiang 1996, S. 251.

191 Vgl. ebd., S. 230-277.

192 Das neue Bildungswesen wurde nach amerikanischem Muster modifiziert, nämlich 6 (4-2) - 6 (3-3 oder 4-2 oder 2-4) - 4 oder 6 (Jahre). Diese Gliederung des Bildungswesens beruft sich auf die physische und psychische Entwicklung des Menschen, nämlich die primäre Stufe der Kindheitsphase im Alter von 6 bis 12, die sekundäre Stufe der Pubertätsphase im Alter von 12 bis 18, die tertiäre Stufe der Jugendzeit im Alter von 18 bis 24. Vgl. Chien Man-chien und Chin Lin-hsiang 1996, S. 279-281. 193 Vgl.ebd. 
1928 wurden diese stark an dem US- amerikanischen Modell angelehnte Bildungsprogramme von zunehmend nationalist- ischen, antikommunistischen und parteidoktrinär bestimmten Bildungsprogramme abgelöst, wodurch sich die staatliche Kontrolle des Bildungswesens wieder verschärfte. 


\subsection{Gestaltung des Bildungswesens unter dem KMT-Regime von 1928 bis 1949}

Einer der fortschrittlichsten Gelehrten, die sich mit westlichen Theorien auseinandersetzten, um die Ursachen für die rückständige Entwicklung Chinas zu finden und zu beheben, war Sun Yat-sen. Er beschäftigte sich nicht nur mit der Revolution, sondern entwarf auch Aufbauprogramme zur Schaffung eines reichen, mächtigen und anerkannten Staates, welche sich auf die Gestaltung des Bildungswesens in der Phase der sog. Erziehungsdiktatur von 1928 bis 1949 auf chinesischem Festland, sogar nach dem Zweiten Weltkrieg auf Taiwan durch das KMT-Regime auswirkten. In diesem Abschnitt werden die Staatslehre Suns und ihre Umsetzung auf das staatliche Bildungswesen untersucht.

\subsubsection{Suns „Drei Prinzipien des Volkes“6}

Als Führer der Revolution gegen das Ching-Kaiserreich war Sun Yat-sen 1912 auch der erste Präsident der Republik China. 1913 übernahm Yuen Shih-kai (1859-1916) das Präsidentenamt, und Sun Yat-sen, seine Anhänger und Teile der Abgeordneten der Nationalversammlung zogen sich nach Kuangtung in Südchina zurück, wo sie eine neue Regierung bildeten und eine zweite Revolution, nun gegen das Regime von Yuen Shih-kai in Peking starteten. Sie kämpften für ein einheitliches modernes China. 1922 wurde seine Aufsatzsammlung unter dem Titel „Aufbauprogramm eines Nationalstaates“ und 1924 sein Programm „Aufbaugrundlage eines Nationalstaates“veröffentlicht. Von Januar bis August 1924 hielt Sun sechzehn Vorträge über seine Programme, um Fragen zur Innen- und Außenpolitik zu beantworten. Diese Vorträge erschienen später unter dem Titel „Drei Prinzipien des Volkes“, Nationalismus, Demokratie und Volkswohl, durch sie sollte China zu einem modernen Nationalstaat umgeformt werden. Die „Drei Prinzipien des Volkes“ stellten nicht nur das einflussreichste, in sich geschlossene Aufbauprogramm Chinas dar, nach 
Meinung von Gottfried-Karl Kindermann beeinflusste die Suns Lehre auch Entwicklungsstrategien im afro-asiatischen Raum, um die Länder von imperialistischer Kolonisation zu befreien und ihre sozialökonomischen Bedingungen zu erneuern. ${ }^{194}$

Ausgangspunkt der „Drei Prinzipien des Volkes“ war die Frage, wie China trotz Übernahme westlicher Kulturgüter dennoch seine kulturelle Identität bewahren könne, damit die Bevölkerung vor der Gefahr einer kulturellen Überfremdung und Entwurzelung gefeit sei. Sun war der Meinung, dass nur eine Vermischung von konfuzianischem Erbe und westlichem Wissen es zustande bringen könne, die Menschen aus ihrer Trägheit zu reißen und das Land vor der Katastrophe zu retten. In seinem Aufbauprogramm brachte er westliches Gedankengut, wie die Lehren von Jean Jacques Rousseau, Baron de Montesquieu, John Mill, Henry George, Karl Marx u. a. mit dem Konfuzianismus zusammen.

\subsubsection{Das Prinzip des Volkstums}

Das chinesische Volk sei zwar das größte in der Welt, nach Suns Sicht aber wie „ein Haufen loser Sand“ - ohne staatsbürgerliche Tugenden und unfähig zur stabilen politischen Selbstbestimmung und -organisation. ${ }^{195}$ China sei eine „Halbkolonie“ und habe damit eine schlimmere Position inne als die sonstigen Kolonien jener Zeit. Seit Ende des 19. Jahrhunderts sei es Schauplatz von Konflikten unter den Großmächten wie Russland, Großbritannien, Japan, Frankreich, den USA und dem Deutschen Reich und genieße selbst keinen Schutz. Seine Situation gleiche einem Fisch, aus dem sich

\footnotetext{
194 Vgl. Gottfried-Karl Kindermann 1963 und 1977, S. 21

195 Vgl. Sun Yat-sen 1969, S. $216 f$.
} 
andere nach Belieben die besten Stücke herausschneiden. ${ }^{196}$

Demgegenüber waren die Staaten im westlichen Europa, welche sich nach der Idee des Nationalstaates verwirklichten, politisch und wirtschaftlich erfolgreich und gelangten in Kürze zu Weltmachtstatus. ${ }^{197}$ Mit dem „Prinzip des Volkstums“ suchte Sun nun eine theoretische Basis zu zeichnen, um zur Gleichberechtigung Chinas zu gelangen. ${ }^{198}$ Darin wurde eine Weltrevolution anvisiert, ein globaler Freiheitskampf der unterdrückten Völker gegen die imperialen Weltmächte. Die drei Hauptziele waren:

(1) ein gleichberechtigter unabhängiger Status Chinas in der Welt, um es vor Fremdherrschaft und Angriffen der Weltmächte zu schützen;

(2) ein gleichberechtigter Status für alle ethnischen Gruppen, um die nationale Einheit Chinas herzustellen;

(3) eine Wiederbelebung und Erneuerung der traditionellen Kultur, um das nationale Bewusstsein der Bevölkerung herauszubilden. ${ }^{199}$

Der Name China bedeutet wörtlich übersetzt „Reich der Mitte“. Bereits diese Bezeichnung verrät das Selbstverständnis der Chinesen ihrer zentralen Rolle in der Zivilisation. Nach traditionellem Verständnis stand China also im Mittelpunkt und an der Spitze einer in hierarchischen Kreisen angeordneten Welt. Symmetrische Beziehungen zu anderen Staaten oder gar asymmetrische Beziehungen zu Ungunsten Chinas waren nach diesem Verständnis undenkbar. Patriotismus sowie der Begriff des Staates im modernen Sinne war demnach in der chinesischen Kulturtradition obsolet

\footnotetext{
196 Vgl. ebd., S. 112.

197 Vgl. Benedict R. Anderson 1988.

198 Vgl. ders. 1963, S. 91-105.

199 Vgl. Gottfried-Karl Kindermann 1977, S. 96-106.
} 
und „Nationalismus“ neu in China. Loyalität hatte gegenüber den Herrschern, den Kaisern sowie der traditionellen Ethik und Kultur $\mathrm{zu}$ herrschen, nicht jedoch gegenüber der „Nation“. Nach Sun bilden fünf Elemente die Basis des nationalen Zusammenhalts: Abstammung, Lebensstil, Sprache, Religion sowie Sitten und Gebräuche. ${ }^{200}$ Die Basis der chinesischen Nation sei indes die Familie, das starke Zusammengehörigkeitsgefühl sei sukzessive von der eigenen Verwandtschaft auf die

Sippe, die Nation und schließlich den Staat zu erweitern. ${ }^{201}$ Sun betrachtete also den Wert der Familie nicht wie die chinesischen Kommunisten als Hindernis auf dem Weg zu einer modernen Gesellschaftsordnung, sondern versuchte eine Verbindung zwischen Familie und Nation herzustellen. Das Nationalbewusstsein sollte über einen Appell an das Familien- und Sippenbewusstsein geweckt werden. Letzteres sollte somit als Grundlage für die geeinte Nation sowie zur Wiederherstellung der chinesischen Morallehre, vor allem der acht Tugenden des Konfuzianismus Loyalität, kindliche Ehrfurcht, Humanität, Liebe und Vertrauen, Gerechtigkeit, Harmonie und Friedensliebe -, dienen. ${ }^{202}$

\subsubsection{Das Prinzip der Volksrechte}

Für die Ausarbeitung des „Prinzips der Volksrechte“ nahm Sun das schweizerische und US-amerikanische System zum Vorbild, nämlich das Recht auf Wahl und Abberufung aller Amtsträger, auf Gesetzesinitiativen und Referenden. ${ }^{203}$ Zur klassischen europäischen Gewaltenteilung in Legislative, Exekutive und Judikative nahm Sun zwei weitere, aus der chinesischen Tradition resultierende Gewalten hinzu, nämlich die staatliche Beamtenprüfung sowie die Kontrolle der Beamten und

\footnotetext{
${ }^{200}$ Vgl. Sun Yat-sen 1969 S.110-112 und Gottfried-Karl Kindermann 1977, S. 91-96.

201 Vgl. ebd., S. 91.

202 Vgl. Sun Yat-sen 1969, S. 108, 173-177 und 178-193.

203 Vgl. ebd., S. 309-311.
} 
öffentlichen Bediensteten. ${ }^{204}$ Doch seien bei der Auslese der Beamten keine Klassenunterschiede mehr zu achten, vielmehr solle der „Weg nach oben“ allen Qualifizierten offen stehen. $^{205}$

Die Entwicklung Chinas zur verfassungsmäßigen Demokratie sollte in drei Phasen verwirklicht werden: zunächst die politische Einheit der Nation durch Militärdiktatur; als Nächstes sollte mittels Erziehungsdiktatur, die Errichtung von Verfassungssystemen und die Ausübung der Bürgerrechte, das Volk an die neue politische Lebensform gewöhnt werden; in der dritten Phase solle schließlich die Demokratie durch die Gründung eines Verfassungsstaates verwirklicht werden. ${ }^{206}$

\subsubsection{Das Prinzip des Volkswohls}

Die „Drei Prinzipien des Volkes“ richteten sich darauf, „Chinas internationale Gleichberechtigung nach außen sowie politisches Gleichgewicht und wirtschaftliche Gerechtigkeit im Innern zu bewirken, um Chinas Fortbestand in der Welt $\mathrm{zu}$ sichern. “207

Sun lehnte die soziale Evolutionsthese ab, nach der die Gesellschaft nach dem Faustrecht, der natürlichen Auslese, funktioniere. Stattdessen übernahm er die konfuzianische Sozialutopie, nämlich die „große Gemeinsamkeit der Welt“ aus dem „Buch der Riten“, und das „Volkswohl“ als anzustrebendes Ziel beim Aufbau eines modernen Staates. ${ }^{208}$ Im Buch der Riten heißt es: „Zu der Zeit, als die großartige

\footnotetext{
${ }^{204}$ Vgl. ebd., S. 311-316; Gottfried-Karl Kindermann 1963, S. 105-112, und ders. 1977, S. 106-113 und 122-126.

${ }^{205}$ Vgl. Sun Yat-sen 1969, S. 313 und Marie-Claire Bergère 1998, S. 370-381.

206 Vgl. Gottfried-Karl Kindermann 1977, S. 120-127.

${ }^{207}$ Vgl. ebd., S. 91.

208 Vgl. Chiang Kai-shek 1953, S. 258-260.
} 
Lehre eingeführt wurde, war die Welt gemeinsamer Besitz. Man wählte die Tüchtigsten und Fähigsten zu Führern; man sprach die Wahrheit und pflegte die Eintracht. Darum ist jeder nicht nur liebevoll seinen nahe stehenden Mitmenschen gegenüber und respektvoll seinen Älteren gegenüber. Die Alten sahen in Ruhe ihrem Ende entgegen; die kräftigen Männer leisteten ihren Beitrag für die Gesellschaft; die Jugendlichen und Kinder wurden gut versorgt und erzogen; die Witwer und Witwen, die Waisen, Kinderlosen, Behinderten und die Kranken hatten ihre Pflege; die Männer hatten ihre Stellung und die Frauen ihr Heim. Die Güter wollte man nicht ungenutzt wegwerfen; aber man suchte sie nicht unter allen Umständen für sich selbst aufzuhäufen. Die eigene Kraft wollte man nicht untätig lassen; aber man arbeitete nicht um des eigenen Vorteils willen. Mit allen Listen und Ränken war es zu Ende; man brauchte sie nicht. Räuber und Diebe, Rebellen und Verräter betrieben ihre Aktivitäten nicht mehr. Darum hatte man zwar draußen Tore; aber man schloss sie nicht ab. Das war die Zeit der großen Gemeinsamkeit der Welt.“209

Sun sah die Ursachen für Reichtum und Stärke Europas nicht nur in deren militärischer Überlegenheit, sondern auch darin, dass die Länder „die Fähigkeiten und Begabungen der gesamten Bevölkerung ausschöpfen und alle Begabungen und Talente an angemessener Stelle nutzbar machen. Die Bauern produzieren Nahrung, die Handwerker stellen Güter zum richtigen Nutzen her, die Kaufleute versorgen die Konsumenten. Jeder erfüllt seine Aufgaben und geht seiner Arbeit nach. Der Zugang zu Wohlstand und Recht ist gleichermaßen möglich. Alle Waren und Güter werden völlig zu großem Nutzen verwendet.“210 Diese Prinzipien sollten nun auch in China angewandt werden.

\footnotetext{
209 Eigene Übersetzung.

210 Zit. nach Sun Yat-sen 1973, S. 84. Eigene Übersetzung.
} 
Obwohl Sun von Marx und Lenin beeinflusst waren, ${ }^{211}$ lehnte er die Klassenkampftheorie ab, sondern baute seine Thesen auf die Kritik des amerikanischen Marxist, Maurice William auf. ${ }^{212}$ William war der Meinung, dass die determinierende Bewegkraft der Geschichte nicht, wie Karl Marx behauptet hatte, die materiellen Produktionsverhältnisse seien, sondern die Sicherung des Lebensunterhalts eines Volkes. Letztere sei die primäre Triebkraft der gesellschaftlichen Entwicklung. ${ }^{213}$ Seine Definition des Volkswohls unterscheide sich aber, so Sun, vom Kapitalismus, indem es langfristig geplante Maßnahmen für die gesamte Wirtschaftsentwicklung des Landes mit einbeziehe und demzufolge nicht kurzfristige Gewinne und Kapitalakkumulation privater Unternehmer fördere. Sun suchte nach Lösungswegen für den Lebensunterhalt der Völker in Bezug auf Ernährung, Kleidung, Wohnung und Verkehrsmittel; ${ }^{214}$ dazu gehören auch Erziehung und Sozialpolitik. Das Ziel war nicht die Veränderung der Klassenhegemonie, sondern die Verwirklichung von der Vorstellung der sozialen Ordnung des Konfuzianismus. Außerdem gebe es keine Klassenkonflikte, denn die meisten Chinesen seien sehr arm, und es gäbe auch keine Großkapitalisten wie in der westlichen Gesellschaft; daher gelte die These vom Klassenkampf nicht. ${ }^{215}$

Sun schlug vor, dass die Landreform und die Entwicklung staatlichen Kapitals vor allem auf zwei Wegen erfolgen solle: durch „gleichmäßige Verteilung des Bodens

211 Zum Hintergrund der Entstehung vom „Prinzip des Volkswohls“ siehe Bergère, Marie-Claire 1998, S. 381-391. Zum Unterschied zwischen Sunyatsenismus und Kommunismus siehe Gottfried-Karl Kindermann 1963, S. 20-49.

${ }^{212}$ Vgl. Sun Yat-sen 1969, S. 313-364. „Das Prinzip des Volkswohls“ berief sich auf die These von Maurice William in seinem Buch „The Social Interpretation of History - A Refutation of the Marxian Economic Interpretation of History“. Erschienen in London 1922. Vgl. ebd., S. 231f. und Paul Myron Anthony Linebarger 1957, S. 137-145.

${ }^{213}$ Vgl. Paul Myron Anthony Linebarger 1957, S. 142-145.

214 Vgl. Sun Yat-sen 1969, S. 365-405.

215 Vgl. ebd., S. 361f. 
und des Reichtums“ und „Beschränkung des Privatkapitals“. Durch die Erhebung von hohen Steuern versuchte er die sozialen und wirtschaftlichen Reformpläne umzusetzen. Der Staat sollte den Wertzuwachs des Besitzes von Grundherren, die nicht selbst auf dem Land arbeiteten, durch gesetzliche Höchstpachten begrenzen. Damit waren die Grundeigentümer gezwungen, Land an den Staat zu verkaufen, und dieser verkaufte es zu günstigen Bedingungen an die bisherigen Pächter. ${ }^{216}$ Als Prinzip der Beschränkung des Privatkapitals dienten die Einführung einer Einkommensteuer, die Verstaatlichung der Bodenschätze, des Lebensmittelgroßhandels, des Transport- und Verkehrswesens und der Banken sowie die unmittelbaren staatlichen Investitionen, bei denen private Investoren unberücksichtigt blieben. $^{217}$ Außerdem sollte China mithilfe von ausländischem Kapital und Experten auf den Weg zur Entwicklung von Wissenschaft und Technik geführt werden, um die Produktivität des Landes zu erhöhen. ${ }^{218}$

Bevor Sun seine Konzepte zum „Prinzip des Volkswohls“ vollständig in Worte fassen konnte, starb er. Die beiden nicht behandelten Teile Erziehung und Sozialpolitik wurden von Chiang Kai-shek im Jahre 1953 in zwei Aufsätzen über die künftige Entwicklung von Bevölkerung und Erziehung sowie soziale Hilfe, Gesundheit und Unterhaltung in Taiwan veröffentlicht. Die Konkretisierung von konfuzianischer Sozialutopie und die Maßnahmen für den Übergang von der bäuerlichen zur industriellen Gesellschaft waren die wesentlichen Inhalte. ${ }^{219}$

\footnotetext{
216 Vgl. ebd., S. 357-359 und 372.

217 Vgl. Gottfried-Karl Kindermann 1963, S. 116-119.

218 Vgl. ebd., S. 118.

219 Vgl. Chiang Kai-shek 1953, S. 191-260.
} 


\subsubsection{Die „Drei Prinzipien des Volkes“ als Grundlage des Bildungswesens}

1928, nach der Vereinigung Chinas, begann die KMT-Regierung damit, das Bildungswesen im Sinne von Suns „Erziehungsdiktatur“ zu zentralisieren, zu vereinheitlichen bzw. unter staatliche Kontrolle zu bringen und nach Vorgabe der Aufbauprogramme eines Nationalstaates von Sun zu gestalten. Zunächst wurde die Parteiideologie zum obersten Ziel des Bildungswesens erhoben und anschließend eine Reihe von Bildungsreformen anhand der Erziehungsthesen von Tsai Yuen-pei und anderen zeitgenössischen Pädagogen geplant. Auch Suns „Drei Prinzipien des Volkes“" sollten umgesetzt werden.

Unter Einfluss der Darwin'schen These von der „Evolution der Menschheit“ hatte Sun 1923 postuliert, dass alle Menschen bildungsfähig seien und durch Erziehung entwickelt werden könnten; ${ }^{220}$ er kritisierte die traditionelle Erziehung, wodurch Jugendliche nur nach sozialem Aufstieg, nach Amtstiteln und höheren Posten strebten, und forderte eine neue Erziehung, durch die Jugendliche zu Experten im Dienst des Gemeinwohls und des Vaterlands ausgebildet werden sollten. ${ }^{221}$ Das heißt, die chinesischen Jugendlichen sollten für die Reform des Landes, für die Revolution, mobilisiert werden. ${ }^{222}$

Orthodoxe KMT-Politiker waren der Meinung, dass die andauernden sozialen Unruhen und Studentenstreiks seit Gründung der Republik auf eine ungenügende Erziehung zurückzuführen seien, und forderten eine „Parteierziehung“ in den Schulen, um den orientierungslosen Jugendlichen klare Werte und Normen zu vermitteln und

\footnotetext{
220 Vgl. Kao Cheng-i 1985.

221 Vgl. Rede von Sun Yat-sen an der Lin-nan Universität (1923), „Studium zur Verbesserung des Gemeinwohls, nicht aber zur Förderung des hohen Beamtentums“ in Sun Yat-sen 1973, S.8 und Liu Li-hui 2000, S. 1-22.

222 Vgl. Wang Bing-chao 1994, S. S. 247-250.
} 
die Bevölkerung auf ein klares, einheitliches Ziel, den Aufbau des Landes, zu lenken. ${ }^{223}$ Die Idee der revolutionären Erziehung wurde Ende der 1920er-Jahre allmählich im staatlichen Bildungssystem umgesetzt.

Chiang Kai-shek hatte 1924, bei der Errichtung der Whampoa Militärakademie in Kuangtung, der Ausbildungsstätte für die Streitkräfte der KMT, das Ausbildungsmodell der sowjetischen Kommunistischen Partei übernommen. ${ }^{224}$ Dies sollte dafür sorgen, dass die Absolventen ihm politisch und militärisch treu ergeben waren. 1926 erklärte die Regierung Parteiideologie zum Schulfach. Als Tsai Yuen-pei 1927 sein Amt als Leiter der „Obersten Erziehungsbehörde“, eine Übergangsbehörde im Bildungsministerium, in Nanking übernahm, verfügte er sogar, dass die Einsetzung des Personals in allen Schulen gemäß der Parteiideologie der KMT geregelt und Letztere als Prüfungsfach eingeführt werden solle. ${ }^{225}$

Parteierziehung wurde 1927 wie folgt definiert: „Unter Führung der KMT soll die Schule zu revolutionärer und nationalorientierter Erziehung hinführen. Anders gesagt, die Zielsetzung der staatlichen Erziehung beruht auf den Grundlagen der KMT, den ,Drei Prinzipien des Volkes', dem ,Aufbauprogramm eines Nationalstaates', der ,Aufbaugrundlage eines Nationalstaates ${ }^{6}$ und der Deklaration und den Zustimmungserklärungen aller landesweiten Abgeordnetenversammlungen. Darauf soll die Zielsetzung unserer Erziehung beruhen, das ist der konkrete Sinn der

\footnotetext{
${ }^{223}$ Vgl. Yu Ben-fa und Hsiung Hsien-chun 1995, S.702f.

${ }^{224}$ Nach der russischen Revolution von 1917 versuchte Lenin eine internationale Organisation mit weltrevolutionärerer Zielsetzung aufzubauen. Moskau wurde zu einem Ausbildungszentrum für junge Kader des Kommunismus aus aller Welt. In diesem Zusammenhang wurde ab 1923 ein chinesischsowjetisches Bündnis geschlossen. Borodin Mikhail Markovic (1884-1952) und General Blyukher wurden nach China entsandt, um die KMT bei der Organisation, etwa beim Aufbau der Whampoa Militärakademie, sowie der Aufstellung und Ausrüstung der Streitkräfte zu unterstützen. Vgl. Jürgen Domes 1969, S. 83-116 und Dieter Heinzig 1978.

${ }^{225}$ Vgl. Yuan Zheng 2002, S. 115-135.
} 
Parteierziehung. ... Wenn wir eine bestimmte Zielsetzung haben, dann können wir die schulischen Lehrinhalte, die zurzeit der Parteiideologie entgegenstehen, auf dieser Basis umstrukturieren, d.h., die neuen Lehrinhalte sollen sich auf die moderne Erziehungstheorie und wissenschaftliche Auseinandersetzung berufen. Zudem kann durch sie die Parteiideologie erweitert und so die Politik der Partei durchgesetzt werden. “226

Während der ersten „Nationalen Erziehungskonferenz“ 1928 in Nanking wurde die Parteierziehung in die Schule thematisiert. Liberale Teilnehmer wie Hu Shih ${ }^{227}$ lehnten diese $a b$, weil sie den Bestrebungen nach „Autonomie der Erziehung“‘228 entgegenstehe. Die Konferenz jedoch stimmte für die Einführung der Aufbauprogramme des Landesvaters Sun Yat-sen in die Schule. ${ }^{229}$ Im Rahmen des Schulediktes 1922 war das aus dem japanischen Bildungswesen übernommene Fach „Persönlichkeitspflege“ in „Staatsbürgerkunde“ umbenannt worden. Den Schülern sollten durch das Studium der „Vier Bücher und fünf Klassiker“ die konfuzianischen

226 „Programme und Bedeutungen der Parteierziehung der KMT-Regierung“ In: Zeitschrift der Erziehung, Vol. 19 (8) 1928. Zit. nach Cheng Wan-chi 2002, S. 62. Eigene Übersetzung.

${ }_{227}$ Hu Shih (1891-1962) studierte zunächst Landwirtschaft, später Philosophie an der Cornell Universität (1910-1914). Er schrieb bei Dewey seine Doktorarbeit über „die Entwicklung der logischen Methode im antiken China" an der Columbia Universität. Als er 1917 nach China zurückkehrte, wurde er Philosophie-Professor an der Universität Peking und geistiger Führer der Bewegung des vierten Mai 1919. 1938 bis 1942 wirkte er als Botschafter der Republik China in den USA und 1957 bei der UNO. 1946 bis 1948 war er Rektor der Universität Peking und 1957 bis 1962 Präsident der „Academia Sinica“ in Taiwan. Er nahm an vielen Aktivitäten für politische und kulturelle Reformen teil und war ein wichtiger Vertreter des Liberalismus und Pragmatismus im modernen China. Vgl. Tong Tekong 2005.

${ }^{228} 1922$ postulierte Tsai Yuen-pei in einen Aufsatz über „die Autonomie der Erziehung“, dass politische Interessen und Ideologien ebenso von der staatlichen Schulerziehung ferngehalten werden sollten wie religiöse Unterweisungen (in: Neue Erziehung. Vol.4 (3) März 1922). Yu Chia-chu forderte die „Rücknahme der Erziehungshoheit“ in seinem Aufsatz „Die Probleme der christlichen Missionarsschulen“, danach wurden alle christlichen Schulen unter staatliche Aufsicht gestellt. Vgl. Su Yun-fung 1994, S. 555-567; Yang Tsui-hua 1978; Lo Tzu 1981, S. 211-234; Cheng Teng-yun 1994 S. 27; Ye Chia-chih 1981, S. 167-182, und Cha Shih-chieh 1984, S. 148-396. Darüber hinaus sollten die Lehrpläne der ausländischen und christlichen Missionsschulen den Grundlagen der chinesischen ministeriellen Bestimmungen angepasst werden. Religion durfte nicht mehr als Pflichtfach eingesetzt werden, und alle religiösen Aktivitäten in der Schule sollten freiwillig sein. Hinzu kam noch die Forderung nach Verstaatlichung der von den Weltmächten und christlichen Missionaren errichteten Hochschulen. Vgl. Liu Huei-hsuan 1986, S. 186-203 und Wu Chia-ying 1990, S. 257-290.

${ }^{229}$ Vgl. Wang Feng-chieh 1998, S. 366; Yu Ben-fa und Hsiung Hsien-chun 1995, S. 703. 
Moralwerte eingeprägt werden. Das Fach gelangte unter dem Einfluss von John Dewey und seinen ehemaligen chinesischen Studenten ins Schulgesetz. Doch blieben die Inhalte der Erziehung und die politische Intention unverändert und wurden lediglich anders formuliert, um damit Hindernisse bei der Durchführung zu minimieren.

Die dritte Plenartagung der KMT im Jahre 1929 beschloss folgende Erklärung: „Die Erziehung der Republik China beruht auf den ,Drei Prinzipien des Volkes“, deren Ziele in der Vervollkommnung des Lebens der Bevölkerung, der Förderung von sozialen Kompetenzen, der Mitwirkung an der Volkswirtschaft und dem Fortbestand der Nation liegen. Dadurch soll die nationale Unabhängigkeit, die Verbreitung der Demokratie, die Entwicklung des Volkswohls und schließlich die Entwicklung zur großen Gemeinsamkeit der Welt vorangetrieben werden. ${ }^{\text {“ }} 30$

Erziehung meinte aber keineswegs nur das theoretische Lernen in der Schule, sondern alle erzieherischen Einrichtungen sollten vom Geist der „Drei Prinzipien des Volkes“ durchdrungen sein, überall sollten ihre Funktionen angewandt werden. Dahinter stand keine systematische Theorie, sondern man versuchte, die gängigen Erziehungskonzepte der damaligen Zeit zusammenzustellen. Obwohl diese oft zusammenhanglos waren und in die Entwicklung Chinas nicht unbedingt hineinpassten, wurden sie in die Bildungsrichtlinien einbezogen; ihre Spur findet man noch 1984 in folgenden Richtlinien zur Erziehung: ${ }^{231}$

„(1) Alle Fächer in der Schule sollen mit dem Geist der ,Drei Prinzipien des Volkes‘ koordiniert werden. Im Zusammenhang mit den Fächern Geschichte und

\footnotetext{
${ }^{230}$ Zit. nach Bildungsministerium (Hrsg.) 1984, S. 2. Eigene Übersetzung.

231 Die Durchführungsprinzipien wurden auf dem vierten Parteitag im Jahre 1931 formuliert. Vgl. Bildungsministerium (Hrsg.) 1984, S. 2.
} 
Geographie soll ihr Sinn vermittelt werden. Im gemeinschaftlichen Leben sollen die Prinzipien einer Demokratie eingeübt werden. Verschiedene Produktionstechniken sollen gelehrt werden, um die Verwirklichung des Prinzips des allgemeinen Wohlstands zu erreichen. Die ,Drei Prinzipien des Volkes' sollen in die Erkenntnisund Moraltheorien integriert werden, damit die Schüler von der Notwendigkeit ihrer Durchführung überzeugt werden können.

(2) Die politische Erziehung soll sich auf das Testament und die Staatslehre des Landsvaters Sun Yat-sen berufen. Die Kinder und Jugendlichen sollen mit den traditionellen Moralvorstellungen - vier entscheidenden Grundsätze ${ }^{232}$ und acht Tugenden des Konfuzianismus - erzogen werden und zugleich eine gewisse Lebenstüchtigkeit erlernen, um damit die Inlandsproduktion zu erhöhen.

(3) Die Sozialerziehung soll das Volk dazu befähigen, die internationalen Ereignisse einordnen und den Sinn der ,Nation“ verstehen zu können. Außerdem soll sie allgemeine Kenntnisse über das Leben in modernen Städten und auf dem Lande vermitteln. Techniken und angewandte Wissenschaften, die für die Verbesserung des Lebensstandards wichtig sind, sollen erlernt werden. Die Erziehung zum Gemeinwohl soll verstärkt werden, öffentliche Anlagen und Naturparks sauber gehalten werden. Des Weiteren soll das Bewusstsein dafür geschaffen werden, alte Menschen zu pflegen, für Arme zu sorgen und Menschen in Not zu helfen.

(4) Die Universitäten und Fachhochschulen sollen außer der wissenschaftlichen Forschungstätigkeit auch angewandte Fachkenntnisse und -kompetenzen vermitteln, um ganz im Dienste des Landes und der Gesellschaft stehen zu können.

(5) Die ,Drei Prinzipien des Volkes‘ sollen zur Grundlage der Lehrerausbildung werden. Deren Aufgabe soll sein, die Lehrkräfte sowohl in moralischen als auch in

\footnotetext{
${ }^{232}$ Die vier entscheidenden Grundsätze des Konfuzianismus sind Sittlichkeit, Höflichkeit, Anstand und Schamempfindung.
} 
akademischen Themen gleichmäßig auszubilden; nach Möglichkeit sollen die Bildungsstätten für die Lehrerausbildung eigenständig sein.

(6) Sowohl Jungen als auch Mädchen sollen das Recht auf Bildung genießen. Die Mädchenerziehung soll ein sanftes Gemüt zum Ziel haben und die notwendigen Fähigkeiten als Mutter vermitteln, damit die jungen Frauen lernen, den familiären und sozialen Erwartungen zu entsprechen.

(7) Alle Arten von Schulen und sozialen Erziehungseinrichtungen sollen die körperliche Ertüchtigung fördern. In der sekundären und tertiären Stufe sollen sich die Schüler auf eine militärische Ausbildung vorbereiten. Das Ziel der körperlichen Ertüchtigung ist jedoch nicht nur, die physische Kondition des Volkes zu stärken, sondern auch, im Volk Disziplin herzustellen.

(8) Der Unterricht im Bereich Landwirtschaft soll zur Verbesserung der Produktionsmethoden in der Landwirtschaft und $\mathrm{zu}$ einem höheren Absatz beitragen. “233

Diese Bildungsrichtlinie zeigt, dass die Schulerziehung in den Dienst der Realisierung der „Drei Prinzipien des Volkes“ gestellt werden sollte. Das Bildungswesen bezog sich nicht mehr auf bestimmte soziale Gruppen, sondern auf das Volk in seiner Gesamtheit. In einer Republik sollten alle Menschen „Herren“ sein, jeder dürfe und solle sich am Staatsgeschehen beteiligen können und die Kompetenz zur Anrufung der Justiz in der bürgerlich-rechtlichen Gesellschaft erwecken. Den mündigen Bürger könne man aber nur, so heißt es weiter, in den Anforderungen der Erziehung zum Staatsbürger mit dem Konzept „Bildung für alle“ schaffen. Eine praxisbezogene Erziehung bzw. körperliche Betätigung (Arbeitserziehung) sollte höher wertgeschätzt werden als zuvor, die Übernahme von naturwissenschaftlichen und technischen

${ }^{233}$ Zit. nach Bildungsministerium (Hrsg.) 1984, S. 4. Eigene Übersetzung. 
Kenntnissen und Methoden aus dem Westen sollte zur Verbesserung der Produktionsprozesse beitragen, um das Ziel vom Wohl des Volkes zu verwirklichen. Die gesamte Bevölkerung sollte einerseits durch Stärkung von Moral und körperliche Ertüchtigung erzogen werden, andererseits aber bedürfe sie auch der westlichen Lehrmethoden, des Geistes der Wissenschaftlichkeit. 


\section{Umsetzung der Bildungspolitik gemäß den „Drei Prinzipien des Volks“ in Taiwan unter dem KMT-Regime von 1945 bis 1987}

Obwohl das KMT-Regime eine Reihe von Reformmaßnahmen plante, konnten diese wegen des Krieges mit Japan, der dauernden sozialen Unruhen sowie der Widerstände der chinesischen Kommunisten nur begrenzt durchgesetzt werden. ${ }^{234}$ Nach dem Rückzug Chiang Kai-sheks und seiner Anhänger im Dezember 1949 nach Taiwan lieferten jedoch die nicht umgesetzten Bildungspläne die Grundlage für die Erstellung seiner Bildungspolitik. Er sah Taiwan indes vor allem als „Versuchsprovinz“ und vorübergehende Basis bei der Rückeroberung des chinesischen Festlandes. Die Schulpolitik orientierte sich daher nicht an der Realität der schulischen Entwicklung, sondern war auf eine imaginäre „schöne“ Zukunft nach der Wiedereroberung Chinas ausgerichtet.

Zu Beginn seines Rückzugs nach Taiwan hielt Chiang Kai-shek eine Reihe von Reden, in denen er die Ursachen für den verlorenen Bürgerkrieg und das Scheitern seiner Politik in Bezug auf Militär, Bildung, Wirtschaft und Gesellschaft reflektierte. ${ }^{235}$ Chiang war der Meinung, dass die politische Erziehung bzw. Parteierziehung der KMT auf dem chinesischen Festland nicht grundsätzlich durchgesetzt worden war und die Kerngedanken der „Drei Prinzipien des Volkes“ den Jugendlichen nicht richtig zu vermitteln gewesen waren, deswegen hätten die Jugendlichen auch ihre Wertorientierung sowie die politische Identifikation mit dem KMT-Regime verloren. ${ }^{236}$ Sie sollten nun in den Vordergrund der Bildungspolitik rücken.

\footnotetext{
${ }^{234}$ Vgl. Julia C. Strauss 1998.

235 Themen waren z.B.: „Das Verhältnis zwischen Erziehung und Revolution sowie den Aufbau des Landes“, „Die Zeit überprüft die Jugend, die Jugend macht die Geschichte“ und „Reorganisation der Erziehung und Veränderung des Volkscharakters“ im Jahr 1951 sowie „Das Land braucht die revolutionäre Jugend, die Jugend braucht die revolutionäre Erziehung“ im Jahr 1952. Vgl. Chin Hsiao-i (Hrsg.) 1981, S. 263-268 und Chiang Kai-shek 1951 (a).

${ }^{236}$ Vgl. Chiang Kai-shek 1951 (b), S. 210f.; Huang Chia-shu 1991, S. 1974f. und Kao Shao-i 1988, S.216.
} 
Die vom KMT-Regime formulierten Zielsetzungen des Bildungswesens, „zum Fortbestand der Nation“, „,zur Unterstützung des gesellschaftlichen Lebens“ und „,zur Unterstützung der Volkswirtschaft und zum allgemeinen Wohlstand“, ${ }^{237}$ wurden im Zuge der Kriegsvorbereitung zu „Erziehung mit Feder und Waffe“, „Bildung des Volksgeistes“ und „Produktions- und Arbeitserziehung“ umformuliert. So sollten die Jugendlichen zu loyalen Parteianhängern der KMT im Kampf gegen die chinesischen Kommunisten, zu Nationalbewusstsein, Vaterlandsliebe sowie $\mathrm{zu}$ tüchtigen Fachleuten im Dienst des Staatsaufbaus erzogen werden. Wie diese Bildungskonzepte von 1945 bis 1987 umgesetzt werden, untersucht dieser Abschnitt.

\subsection{Militarisierung der Erziehung}

Bereits 1885 war unter Einfluss der Selbststärkungsbewegung in Peking eine Heerschule gegründet worden, die sich an preußischen Ausbildungsmethoden orientierte. $^{238}$ Und nach dem Sino-japanischem Krieg (1898) sowie dem Boxeraufstand (1900), bei denen die Verluste auf die körperlichen Schwächen der Bevölkerung zurückgeführt wurden, nahm man Sport und Militärausbildung - auch nach dem Vorbild des japanischen Bildungswesens ${ }^{239}$ - in die Erziehungskonzepte im Jahre 1903/04 auf. Das Ziel war nicht nur, dass die Schüler militärische Kenntnisse wie Schießen erwarben und durch körperliches Training und hygienische Erziehung gestählt werden sollten, sondern auch, dass sie soldatische Tugenden wie Vaterlandsliebe, Geduld, Disziplin, Brüderlichkeit, Opferbereitschaft usw. vermittelt bekamen. $^{240}$

\footnotetext{
${ }^{237} \mathrm{Vgl}$. 5.5.2. in dieser Arbeit.

238 Vgl. Hwang Jin-lin 2004 S.177-183.

239 Die 1902 von Cai E und Chiang Bei-li ins Chinesische übersetzten Konzepte der japanischen Militarisierung wurden zur Basis der militärischen Erziehung in China. Vgl. Chyu Li-ho 1984 (b), S. 27-52.

${ }^{240}$ Vgl. Chyu Li-ho 1984 (b), S. 32-38.
} 
Die Pfadfindererziehung - eine eigenlich freiwillige außerschulische Aktivität- wurde zum Teil der zwangshaftigen staatlichen Militärerziehung $1928^{241}$ umgesetzt, um die Jugendlichen von Demonstrationen und Studentenstreiks fernzuhalten. ${ }^{242}$ Im Zuge der „Bewegung Neues Leben“ (1934-1949) ${ }^{243}$ bzw. während des Zweiten Weltkriegs erreichte die Militarisierung der Schule ihren Höhepunkt, sie diente nun der Stärkung von Kampfeswillen und Opferbereitschaft für das Vaterland. Militärwesen, Gymnastik, Sport sowie die „Drei Prinzipien des Volkes“ wurden in der Sekundarstufe II als Pflichtfächer mit mindestens vier Wochenstunden eingeführt. Die Lehrinhalte im Bereich der Allgemeinbildung, wie Geschichte, Geographie, Naturwissenschaft und Technik, sollten mit den Inhalten militärischer Erziehung verbunden werden. Doch wurde die Militärerziehung aufgrund des Bürgerkrieges mit den chinesischen Kommunisten und mangels finanzieller Unterstützung abgebrochen. Wie schon zuvor kam es immer häufiger zu Studentenunruhen, und schließlich verlor das KMT-Regime gänzlich die Kontrolle über die Schulen, später über ganz China.

Aus voriger Erfahrung heraus richtete das KMT-Regime auf Taiwan besondere Aufmerksamkeit auf die politische und militärische Erziehung, um die Schüler und

\footnotetext{
241 Die seit 1907 vom britischen General Robert Baden-Powell propagierte Pfadfindermethode mit der Zielsetzung, die Entwicklung junger Menschen durch Übernahme von Verantwortung zu fördern, wurde 1912 in China eingeführt. Zu Beginn wurde sie nur von christlichen Missionarsschulen, vor allem in Shanghai, gefördert und allmählich auf andere Provinzen ausgeweitet. 1926 wurden die Pfadfinderverbände der KMT angegliedert. Vgl. Chin Sui-ling 2004 und Huang Chu-li 2005, S. 99-138.

${ }^{242}$ Vgl. Bildungsministerium (Hrsg.) 1974, S. 1371 und Wu Chia-ying 1990, S.45-57.

2431934 hatte Chiang Kai-shek mit der „Bewegung Neues Leben“ zur moralischen und geistigen Erneuerung des Volkes und zur Rückkehr zu den konfuzianischen Tugenden aufgerufen. Vgl. Chiang Kai-shek 1934. Im Zusammenhang nationaler und faschistischer Zeitströmung stellte die Bewegung einen Versuch dar, eine Verbindung zwischen militärischen, faschistischen, christlichen und traditionell konfuzianischen Elementen herzustellen und für eine neue politische Situation nutzbar zu machen, um China auf der Basis seiner eigenen Tradition als moderne, gleichberechtigte Nation zu etablieren. Das brachte der KMT nicht nur neue politische Impulse, sondern führte auch dazu, dass sich die von der „Bewegung zur Kulturerneuerung 1919“ beeinflussten chinesischen Intellektuellen noch mehr als zuvor von der KMT abwandten. Während des Zweiten Weltkrieges diente die Massenmobilmachung dazu, Antikommunismus und Widerstand gegen die japanische Invasion zu verstärken. Vgl. Wang Chung-fu 1981, S. 2027-2114; Wang Shou- nan 1981, S. 2027-2051; Hwang Jin-lin 1998, S. 163-203; James C. Thomson 1969, S. 152 und Lloyd E. Eastman 1974.
} 
Studenten unter Kontrolle zu bringen und Studentenunruhen im Keim zu ersticken.

Desweiteren sollten die Jugendlichen zur Avantgarde des Krieges für die

Rückeroberung des chinesischen Festlands erzogen werden. ${ }^{244} 1951$ wurde der Staatssicherheitsrat unter Leitung von Chiang Kai-sheks Sohn, Chiang Ching-kou, ${ }^{245}$ gegründet und auf diesem Wege ein militärisch-autokratisches Herrschaftssystem in Taiwan aufgebaut. ${ }^{246}$ Ein Jahr später wurde „The China Youth Corps“ (CYC) gegründet, ${ }^{247}$ um die KMT zu reorganisieren und die Bildungsprogramme von Chiang Ching-kuo, die er auf der Basis seiner Erfahrungen mit der sowjetischen Jugendliga entwickelt hatte, zu unterstützen. ${ }^{248}$ Beim Vorläufer des CYC, dem „San-min chu-i Jugendverband” (1938-1949) auf dem chinesischen Festland, wurden Jugendliche zu Kämpfern für Staat und Partei herangebildet. Der CYC ähnelte in vielerlei Hinsicht der Ballila in Italie, Hitlerjugend in Deutschland sowie den Kommunistischen Jugendverbänden in der Sowjetunion und in der Volksrepublik China. ${ }^{249}$

1951 wurde die militärische Ausbildung von Chiang Kai-shek zunächst in den

\footnotetext{
244 Vgl. Sun Pang-cheng 1976, S. 27-49.

245 Chiang Ching-kuo studierte 1925-37 in nach Moskau und wurde später Mitglied der Kommunistischen Jugendliga. 1943 begann er seine Karriere in der KMT. In den 1950er-Jahren wurde er Leiter der Politabteilung im Verteidigungsministerium und Chef der Geheimpolizei in Taiwan sowie Mitglied des Reformkomitees der KMT und war damit einer der mächtigsten Personen Taiwans. 1969 wurde er Stellvertretender Ministerpräsident, 1971 Ministerpräsident, 1978-1988 Präsident. Als Präsident läutete Chiang die Demokratisierung und Taiwanisierung der Politik ein und schuf den Rat für wirtschaftliche Planung und Entwicklung, um die ökonomische Entwicklung in Taiwan zu koordinieren.

${ }^{246}$ Vgl. Hsu Fu-ming 1986, S. 110f.; Sun Chiang-lin 1961, S. 21 und Wang Liang-ching 2003.

247 Die Legitimation der „China Youth Corps“ war von Beginn an sehr umstritten. Ab 1960 wurde die Aufgabe der militärischen Erziehung in der Sekundarstufe II von der Abteilung Militärinstruktion des Bildungsministeriums übernommen. Im Laufe der Zeit verlor der CYC seine politische und militärische Funktion. Nach den 1970er-Jahren wandelte er sich zu einem Veranstalter von Freizeitunternehmen der taiwanesischen und übersee-chinesischen Jugend. 1982 wurde er als Gesellschaft registriert. Vgl. Chen Yao-hong 1992, S. 252 f.

248 Chiang Ching-kuo hatte von 1925 bis 1937 eine kommunistische Bildung erhalten und an der sowjetischen Kommunistischen Jugendliga teilgenommen. Ab 1950 war er für den Nachrichtendienst sowie die politische und militärische Erziehung auf Taiwan zuständig. Vgl. Lee Wei-sung 2005, S. 383-384.
}

249 Vgl. Wang Chih-hsiang 2008, S.157-170. 
Pädagogischen Schulen eingeführt. ${ }^{250}$ Ab 1953 sollten alle Hochschulabsolventen ein Jahr lang, später zwei Jahre lang, militärisch geschult werden. Ab 1954 wurde die Militärerziehung bereits in allen Schulen in der Sekundarstufe II vier Stunden pro Woche praktiziert ${ }^{251}$ und Offiziere als Lehrkräfte berufen. ${ }^{252}$ In der Sekundarstufe I wurde eine Pfadfindererziehung praktiziert, um die Schüler zu disziplinieren und die Basis für die militärische Ausbildung zu schaffen. ${ }^{253}$ Die Schulen, Universitäten und Fachhochschulen waren wie Kasernen organisiert. Die Schüler bzw. Studenten wurden in mittlere oder kleine Einheiten des CYC aufgegliedert; jede führende Person war gleichzeitig der Führer dieser Einheiten. Jugendaufsichten wurden eingesetzt, die für die Instruktionen bei den militärischen Übungen der Jungen, die Vermittlung von Krankenpflege und Rettungsmethoden bei Mädchen sowie für die politische Bildung zuständig waren; ${ }^{254}$ gleichzeitig überwachten sie die Gesinnung und Persönlichkeitsentwicklung der Schüler und Studenten. ${ }^{255}$

Militärische Unterweisung und Disziplin wurden Teil des schulischen Unterrichts, ebenso Pflichtbewusstsein, Höflichkeit, Etikette und Schamgefühl sowie Vaterlandsliebe, mit dem Ziel, eine mögliche Verbreitung des Kommunismus sowie Unruhen in den Schulen auszuschließen. Es gab einen Paradeplatz, Fahnenmasten und Parademärsche auf dem Schulgelände, wie schon unter der Herrschaft des KMT-Regime in den 1930er- und 40er-Jahren. Die Nationalhymne wurde während der täglichen Morgen- bzw. Nachmittagsversammlung sowie bei fast allen offiziellen Gelegenheiten gesungen, am Schuleingang standen Statuen von Konfuzius oder Chiang

\footnotetext{
${ }^{250}$ Vgl. Bildungsministerium (Hrsg.) 1957, S. 298.

251 Vgl. ders. 1974, S. 1085.

252 Vgl. ebd., S. 1372-1378.

${ }^{253}$ Vgl. Hwang Jin-lin 2009, S. 141-143.

${ }^{254}$ Vgl. Bildungsministerium (Hrsg.) 1957, S. $15 f$.

${ }^{255}$ Die Konzepte der militärischen Erziehung und Instruktion von Chiang Kai-sheks Truppe standen stark unter dem Einfluss des japanischen und deutschen Militarismus. Vgl. Hwang Jin-lin 2009, S. $114-119$
} 
Kai-shek, an den Wänden der Klassenzimmer hingen Bilder von Sun Yat-sen, Chiang Kai-shek sowie dem jeweiligen Präsidenten und Vizepräsidenten. ${ }^{256}$ Lehrer und Schüler sollten sowohl in wie auch außerhalb der Schule in militärischer Weise miteinander umgehen, die Schüler eine militärische Uniform tragen und dem Klassenlehrer ein Wochenbuch ${ }^{257}$ abgeben. Durch die militärische Erziehung wurden die Jugendlichen unter politische Kontrolle gebracht. ${ }^{258}$ Obwohl der Plan eines Krieges gegen die chinesischen Kommunisten seit Mitte der 1960er-Jahre langsam aufgegeben wurde, wurde die militärische Erziehung bis in die 1990er-Jahre fortgesetzt, um die Stabilität der Einparteiendiktatur und die soziale Ordnung abzusichern.

\subsection{Die „Bildung des Volksgeistes“}

Die Bedeutung der „Bildung des Volksgeistes“ für die Vereinigung der Nation und die Bildung der nationalen Identität wird im „Prinzip des Volkstums“ Suns thematisiert. Eine konkrete Umsetzung erlebte das Konzept während der nationalistischen und faschistischen Zeitströmung in den 1930er- und 40er-Jahren, vor allem im Zusammenhang mit der „Bewegung Neues Leben“ (1934-1949), welche durch soziale Erziehung eine radikale Änderung bei der Bevölkerung in Bezug auf Benehmen, Essen, Kleidung, Wohnen etc., eine Kultivierung erreichen wollte, ${ }^{259}$ etwa mittels Studium der chinesischen Klassiker und Belebung der traditionellen Werte und Normen, womit wieder an die alte Kultur und Tradition angeschlossen werden sollte. ${ }^{260}$ Die Verbindung konfuzianischer und militärischer Tugenden in

\footnotetext{
256 Vgl. Bildungsministerium (Hrsg.) 1974, S. 1082.

257 Am jeden Montag mussten die Schüler in der Sekundarstufe ihrem Klassenlehrer einen Bericht über bedeutende Neuigkeiten und besonderen Erlebnisse der vergangenen Woche einreichen.

258 Vgl. Chiang Kai-shek 1952 (b) und (c); Ku Tao-chung 1985, S. 3; Wang Pei-lin 2001, S. 99-102 und Lee Tai- han 2002, S. 95-116.

259 Vgl. Wang Shou-nan 1981, S. 2073-2082 und Lloyd. E. Eastman 1976, S.191-210.

260 Vgl. Jürgen Domes 1969, S. 552f.
} 
dieser Bewegung stellte einen Versuch dar, traditionelle Elemente für eine neue politische Situation nutzbar zu machen, um China auf der Basis seiner eigenen Tradition zu einer modernen gleichberechtigten Nation zu machen. Während des Sino-Japanischen Krieges sicherte diese Massenmobilmachung gleichfalls die Unterstützung der Volksmassen für die KMT-Herrschaft, d.h. sie diente auch dazu, die Volksmassen zum Anti-Kommunismus und Widerstand gegen die japanische Invasion zu erziehen. ${ }^{261} 1935$ hatten zehn kulturkonservative Gelehrten „Deklaration zum Aufbau einer auf China basierenden Kultur“" veröffentlicht, damit die Wiederbelebung des Konfuzianismus auf der These von „die chinesische Weltanschauung als Grundlage und das westliche Wissen zum praktischen Gebrauch“ ${ }^{\text {zu fordern. }}{ }^{262}$ Sie waren der Meinung, dass die Wiederbelebung der konfuziansichen Werte und Normen nicht nur zum Beitrag des Wiederaufbaus der sozialen Ordung Chinas, sondern auch des Selbsbewusstsein der Chinesen, und auch der Modernisierung des Chinas. Diese Einstellung zum Konfuzianismus und zum Aufbau eines modernen Chinas wurde mit der Einseiderung dieser Gelehrten in Taiwan fortgesetzt. Diese These wurde zum wichtigsten Teil der Bildungspolitik in Taiwan einbezogen. Hinzu kamen die Erziehung $\mathrm{zu}$ einer einheitlichen Nationalsprache, die Unterweisung in konfuzianischer Lehre, die antikommunistische Kulturpolitik sowie die „Bewegung zur Bewahrung und Restauration chinesischer Kultur".

\subsubsection{Die Vereinheitlichung der Verkehrssprache}

In China wurde nur die Schriftsprache vereinheitlicht, die Umgangssprache jedoch nicht; innerhalb des riesigen Landes gibt es zahlreiche Völker mit vielfältigen

\footnotetext{
261 Vgl. Wang Shou-nan 1981, S. 2027-2114.

262 Vgl. Arif Dirlik 1975, S. 945-980.
} 
Dialekten. ${ }^{263}$ Beeindruckt vom Erfolg der japanischen „Bewegung der Nationalsprache“ ${ }^{264}$ schlug der chinesische Gelehrte Wu Ju-lun (1840-1903) bei Erstellung des Schulgesetzes im Jahr 1903/04 Chang Pai-hsi vor, die Amtssprache, den sog. „Peking-Dialekt“, als Schulsprache zu verwenden. ${ }^{265}$ Nach Gründung der Republik wurde dieses Konzept weiter verfolgt, 1919 die „Kommission für die Vereinheitlichung der Nationalsprache“ gegründet und um 1920 der Peking-Dialekt zur Nationalsprache erklärt. ${ }^{266}$ Während der „Bewegung des Vierten Mai 1919“ wurde zudem die Vereinfachung der klassischen Schriftsprache, die nur von Angehörigen der Honoratiorenschicht verwendet werden konnte, gefordert. Die sog. „Bewegung für umgangssprachliche Literatur“ wollte die Umgangssprache zur öffentlichen Schriftsprache erklären. Sie setzte sich in Literatur und Presse schließlich auch durch, sodass die schriftliche Propaganda revolutionärer Bewegungen die breite Bevölkerung erreichte. ${ }^{267}$ Hinzu kam noch der Vorschlag zur Vereinfachung der chinesischen Schrift von Chien Hsuan-tung (1887-1939). Sein Reformvorschlag stand aber in den 1930er-Jahren im Widerspruch zur gerade vom KMT-Regime ausgerufenen „Bewegung zur Restauration der chinesischen Kultur und Tradition“ und wurde nicht akzeptiert, doch wurde er von anderen Linguisten weiter verfolgt. 1958 hieß die Volksrepublik China den „Plan zur Vereinfachung der chinesischen Schrift“ gut, seitdem wird die vereinfachte Schrift als offizielle Schrift dort praktiziert. ${ }^{268}$

Nach Suns „Prinzip des Volkstums“ ist die Landessprache die natürlichste Kraft bei der Integration und bildet die Basis für die Bildung eines Nationalstaates. ${ }^{269}$ Sie soll

\footnotetext{
263 Vgl. Masahisa Shimura 1993, S. 54f.

264 Vgl. Chyu Li-ho 1984 (a), S. $146 f$.

265 Vgl. Hung Wei-jen 1992, S. 228 und Fan Shih-tuo 1965, S. 17-50.

266 Vgl. Chan Wei 1992, S. 99.

267 Vgl. Wang Er-ming 1982, S. 13-45.

268 Vgl. Huang Kuo-lu 2007 S.53-90 und Huang Kuo-feng 1987.

269 Vgl. Sun Yat-sen 1969, S. 110-112.
} 
dazu dienen, standardisierte Verhaltensweisen $\mathrm{zu}$ entwickeln und darüber die herrschenden politischen und wirtschaftlichen Verhältnisse sowie die sozialen Werte und Normen zu akzeptieren. Um Schwierigkeiten bei der Verbreitung des Peking Dialekts zu begegnen, wurde ein standardisiertes Phonetiksystem, das auf der traditionellen chinesischen Transkription basierte, für chinesische Schriftzeichen bestimmt und 1918 vom Bildungsministerium erlassen. 1928 wurde es von der KMT-Regierung anerkannt. ${ }^{270}$ Die Umschrift basierte auf der Phonetik des römischen bzw. lateinischen Alphabets und war 1926 von einer Gruppe chinesischer Linguisten unter Chao Yuen-jen (1892-1982) erstellt worden. ${ }^{271}$ Seit 1958 wurde ein weiteres System für die Umschrift des Chinesischen, das Pinyingsystem, in der Volksrepublik China entwickelt. ${ }^{272}$

Der erste Schritt der Erziehung nach dem Konzept des „Prinzips der Volksrechte" bestand in der Beseitigung des Analphabetismus. ${ }^{273}$ Obwohl die Schulpflicht offiziell seit Beginn des 20. Jahrhunderts in China eingeführt war, kam bis zum Ende des Zweiten Weltkriegs ein großer Teil der Bevölkerung nicht in den Genuss einer Schulbildung, infolge dessen gab es zahlreiche Analphabeten. Auch die Vereinheitlichung der Verkehrssprache konnte unter der KMT-Regierung nicht landesweit durchgesetzt werden. Erst nach dem Zweiten Weltkrieg wurden die Verbreitung der traditionellen Schrift sowie eine einheitliche Nationalsprache auf Taiwan radikal durchgesetzt.

\footnotetext{
${ }^{270}$ Bereits während der Ming-Dynastie entwickelten Jesuiten wie z.B. Matteo Ricci von 1583-1610 ein Phonetiksystem für die Transkription von chinesischen in römische bzw. lateinische Silben. Vgl. Kao Ming 1978, S. 314f.

${ }^{271}$ Vgl. Li Chin-hsi 1931.

272 Vgl. Tsao Ruey-tai 2007 S. 10.

273 Vgl. Chiang Kai-shek 1953 S. $228 f$.
} 


\subsubsection{Bewegung der Dejapanisierung bzw. Sinisierung}

Nach der fünfzigjährigen Trennung zwischen Taiwan und dem chinesischen Festland war eine breite Kluft zwischen zwei Gruppen entstanden, den Taiwanesen und den neu hinzugekommenen Chinesen, die auf sprachliche Unterschiede und historische Erfahrungen zurückgeht. Es gab nur wenige Chinesen in der neuen Regierung, die die taiwanesische oder die japanische Sprache beherrschten. Umgekehrt gab es kaum Taiwanesen, die des Hochchinesischen mächtig waren. Es fehlte folglich an Kommunikationsmöglichkeiten zwischen beiden Gruppen. ${ }^{274}$ Während Taiwan japanische Kolonie war, standen die Chinesen unter dauernder Invasionsdrohung der Japaner. Sowohl die Beamten als auch die einfachen Bürger hatten eine feindselige Haltung Japanern gegenüber und eine starke Abneigung gegen die japanische Kultur. Als die chinesischen Beamten in Taiwan ankamen, projizierten sie ihre Erfahrungen mit den Japanern auf die Taiwanesen und betrachteten sie entweder als „ehemalige Kollaborateure Japans“ oder als „Sklaven der Japaner“. 275

Nach der Sicht der chinesischen Beamten sollten die Taiwanesen vom Einfluss Japans befreit und $\mathrm{zu}$ „richtigen Chinesen“ mit hochchinesischer Umgangssprache und Schrift sowie der entsprechenden kulturellen und nationalen Identität umerzogen werden, also genau andersherum als zur Zeit der „Bewegung der Japanisierung“ vor dem Zweiten Weltkrieg auf Taiwan. ${ }^{276} \mathrm{Ab} 1946$ war die Veröffentlichung japanischer Bücher, Zeitungen und Zeitschriften, anschließend auch die Verwendung der japanischen Sprache in der Öffentlichkeit verboten. ${ }^{277} 1946$ wurde das „Komitee zur Verbreitung der Nationalsprache auf Taiwan“ eingerichtet, um Richtlinien sowie

\footnotetext{
274 Vgl. Cheng Tzu 1994 und Wang Chih-ping 2002, S. 66.

275 Vgl. Wang Chih-ping 2002, S. 60.

276 Vgl. Huang Ying-che 2007, S. 17 und He Ching-chin 1980, S. 35.

277 Vgl. Yang Rong-cong 1992, S. 18-24; Wan I-ting 1991, S. 53 und Hong Rui-chong 1998, S. $147 \mathrm{ff}$. 
Lehrbücher und Wörterbücher zu erstellen. ${ }^{278}$ Zugleich wurden Anstalten für die Verbreitung der hochchinesischen Sprache in allen Städten errichtet, die öffentlichen Schulen sollten ebenfalls diesen Zweck erfüllen.

Die Schulen bekamen chinesische Namen, und auch das Schulsystem wurde chinesisch; zudem war eine Gleichbehandlung von Taiwanesen und Chinesen angestrebt. Alle schulischen Lehrinhalte waren an der politischen Ideologie der KMT ausgerichtet, den „Drei Prinzipien des Volkes“: ${ }^{279}$ „Staatsbürgerkunde und Tugenden“ wurde nun statt „Persönlichkeitspflege“ unterrichtet, Sprache, Geschichte und Geographie bezogen sich auf China und nicht länger auf Japan, Sport fand anstelle japanischer Kampfkunst und Gymnastik statt, um das Nationalbewusstsein und die Staatsidee bei den Schülern zu verankern. ${ }^{280}$ So wurden die Spuren der japanischen Kultur auf Taiwan in kurzer Zeit verwischt.

\subsubsection{Bewegung zur Verbreitung der Nationalsprache}

Mit der Durchsetzung der Schulpflicht sowie der Einrichtung von Unterricht für erwachsene Analphabeten wurde 1956 die „Bewegung zur Verbreitung der Nationalsprache“ ins Leben gerufen. ${ }^{281}$ Die Bildungsbehörde erließ hierzu ein Edikt; gleichzeitig wurde die Verwendung des Taiwanesischen in der Schule verboten und die Einwanderer vom chinesischen Festland als Lehrer für Hochchinesisch eingesetzt. Sie machten nach 1949 zwar nur etwa 13\% der Bevölkerung aus, jedoch gab es unter ihnen sehr viele gebildete Gelehrte oder Studenten, die als Lehrkräfte an den Schulen eingesetzt werden konnten. Das Problem des Lehrkräftemangels wurde dadurch

\footnotetext{
${ }^{278}$ Vgl. Chang Po-yu (Hrsg.) 1974, S. 34-65.

279 Vgl. Bildungsbehörde unter den Verwaltungsbehörden des Gouvernements der Provinz Taiwan (Hrsg.) 1955, S. 8 und 29.

${ }^{280}$ Vgl. Ye Hsien-chun 1993, S. 77.

281 Vgl. Shih Ying-chun 1984.
} 
gemindert, aber die chinesischen Flüchtlinge begannen schnell damit, kulturell auf Taiwan zu dominieren. ${ }^{282}$ Zugleich erhielt der Nachwuchs der chinesischen Flüchtlinge durch diese Sprachpolitik mehr Vorteile im Bildungszugang als die Taiwanesen. ${ }^{283}$

\subsubsection{Bewegung für die Bewahrung und Restauration der chinesischen Kultur}

Seit dem Mittel der 1960er-Jahre rief das KMT-Regime zu einer „Bewegung für die Bewahrung und Restauration der chinesischen Kultur" auf. Im diesen Abschnitt sollen historischer Hintergrund und Inhalte dieser Bewegung sowie ihr Einfluss auf die schulische Erziehung vorgestellt werden.

\subsubsection{Historischer Hintergrund der „Bewegung für die Bewahrung und Restauration der chinesischen Kultur"6}

(1) Die Debatte um Einführung der „Wissenschaft“" und Technik in China bzw. Taiwan

Wie bereits erwähnt, studierten zahlreiche chinesische Studenten infolge des Kulturaustauschs Ende des Ching-Kaiserreichs in unterschiedlichen westlichen Ländern und erfuhren dort eine ganz andere Dimension von Wissenschaft, ,ein System von Kenntnissen und Forschungsmethoden durch einen logischen, sachlichen und experimentierenden Untersuchungsvorgang““. ${ }^{284}$ Während der „Bewegung des Vierten Mai 1919“ forderten fortschrittliche Gelehrte, die westlichen Konzepte von Wissenschaft, Demokratie und Autonomie der Forschung zu übernehmen, und propagierten, dass China dadurch zu einem wohlhabenden und starken Land geführt werden sollte. Daraufhin entstand eine Debatte mit den Kulturkonservativen pro und

\footnotetext{
282 Vgl. Chen Mei-ju 1996, S. 75f. und 89-92.

283 Vgl. Tsai Ming-hsien 2009, S. 45.

284 Vgl. Su Yun-fung 1981, S. 21-23.
} 
contra „Verwestlichung“: Liang Shu-ming, ${ }^{285}$ Liang Chi-chao und Chang Chunmai ${ }^{286}$ waren Verteidiger der chinesischen Lebensart und Kultur, Hu Shih und Ting Wen-chiang ${ }^{287}$ als Anhänger des Pragmatismus John Deweys und des Positivismus Befürworter einer Übernahme der westlichen Wissenschaft und Kultur. Chen Tu-hsiu ${ }^{288}$ hingegen vertrat die Lehre des marxistischen Materialismus, sein Ziel war, die Grundlage zur Einführung der „Wissenschaft“ und des Marxismus durch Zerstörung der Tradition zu schaffen. ${ }^{289}$ In der Debatte wurde über die Überlegenheit der ,spirituellen Kultur“ Chinas gegenüber der „materiellen Zivilisation“ des Westens gestritten, über die Stellung von Wissenschaft zu Lebensanschauung und Metaphysik, über die Beziehung der „inneren“ zur „äußeren Welt“ sowie über die Bedeutung eines intuitiven und apriorischen Erkenntniswegs gegenüber einem positivistischen und pragmatischen. $^{290}$

Mit der US-Entwicklungshilfe und der Durchführung von Wirtschaftsplänen wandelte

\footnotetext{
2851921 veröffentlichte Liang Shu-ming (1893-1988), Vertreter des Neokonfuzianismus und Professor für indische und chinesische Philosophie an der Universität Peking, seine vergleichenden Studien über „Östliche und westliche Kultur und ihre Philosophie“. Er vertrat den Standpunkt, dass eine verjüngte chinesische Zivilisation die Grundlage der zukünftigen weltweiten Zivilisation bildet. Vgl. Lee Eun-jeung 2003, S. 551- 561.

${ }^{286}$ Liang Chi-chao und sein Schüler Chang Chun-mai (1887-1969) hatten nach dem Ersten Weltkrieg Europa bereist und waren erschüttert darüber, wie Naturwissenschaften und Technologie die Menschheit und die Welt in die Katastrophe geführt hatten. Liang veröffentlichte 1919 eine Reihe von Reiseberichten über seine Erfahrungen in Europa. Chang Jun-mai beschäftigte sich während seines Aufenthalts in Paris mit Henri Bergson und später in Jena mit Rudolf Christoph Euckens und versuchte eine Synthese zwischen Neokonfuzianismus und Neoidealismus herzustellen. Chang strebte seit den 1930er-Jahren einen Verfassungsstaat in China an. Er gründete 1932 die Nationale Sozialistische Partei Chinas und hatte teil am Entwurf der chinesischen Verfassung 1946. Vgl. Roger B. Jeans, Jr. 1997 und Chen Ta-hwa 1997.

287 Ting Wen-chiang (1887-1936) studierte 1911 Geologie und Zoologie an der Glasgow Universität in England. 1921 gründete er die „Gesellschaft der Geologie in China“ und war 1925 Direktor von „Shanghai Commercial Bureau“. Seit 1931 war er Geologie-Professor an der Universität Peking und seit 1934 Mitglieder der „Academia Sinica“. Vgl. Hu Shih 1960.

${ }^{288}$ Chen Tu-hsiu (1879-1942), seit 1917 Dekan der Geisteswissenschaftlichen Fakultät der Universität Peking, propagierte seit 1915 in der ,Zeitschrift der Jugend“, später umbenannt in „,Neue Jugend“, die Erneuerung des Volkes durch Erneuerung der Kultur. In der „Neuen Jugend“ wurden die verschiedenen Geistesströmungen aus dem Ausland erläutert. Chen ist auch Mitbegründer der Kommunistischen Partei Chinas 1920. Vgl. Chen Kao-xiang 1979, S. 513-525.

289 Vgl. Werner Meißner 1993, S. 125-143.

290 Vgl. Werner Meißner 1994, S. 200f. und Chang Yuh-fa 1977, S. $314 f$.
} 
sich die taiwanesische Gesellschaft allmählich von einer bäuerlichen in eine industrielle Gesellschaft. Gleichzeitig drang die US-amerikanische Kultur mehr und mehr in die taiwanesische Erziehung ein. Vor allem begann die KMT-Regierung am Ende der 1950er-Jahre mit dem Aufbau der beruflichen Ausbildung sowie der Ausweitung der naturwissenschaftlichen Bildung, Forschung und Ausbildung. ${ }^{291}$

Die Annäherung an die westliche bzw. US-amerikanische Kultur wurde indes von einigen Theoretikern als Abgrenzung von der eigenen Tradition gewertet und verurteilt, da sich die naturwissenschaftliche Bildung im Widerspruch zur moralischen Erziehung bzw. „Bildung des Volksgeistes“ befände. ${ }^{292} \mathrm{Hu}$ Shih griff in seiner Rede über „Social Changes Necessary for Science Development“ auf der „Konferenz über naturwissenschaftliche Erziehung in Ostasien“ die amerikanische „Agency for International Development“ scharf an. ${ }^{293} \mathrm{Hu}$ war der Meinung, dass „Wissenschaft“ nicht mit „Technik“ oder „Materialismus“ gleichzusetzen sei. Damit sich die „Wissenschaft“ in der östlichen Kultur wirklich verwurzeln könne, müsse man zunächst die Philosophie der westlichen Kultur kennenlernen. ${ }^{294}$ Aus heutiger Sicht erscheinen Hus Ausführungen selbstverständlich, aber damals wurde seine Rede als soziale Gefahr betrachtet. Nach Veröffentlichung der Rede brachten über zweihundert Aufsätze Kritik dagegen vor. ${ }^{295}$ Im Februar 1962 starb Hu, doch die Debatte ging weiter. In einer Gedenkschrift für ihn in der progressiven und einflussreichen Zeitschrift „,Wen-hsing ““296 schrieb der Historiker Li Ao (geb. 1935), dass man Kultur als Ganzes betrachten müsse, deswegen sei es unmöglich,

\footnotetext{
${ }^{291}$ Vgl. 6.3. in dieser Arbeit.

292 Zeitungsartikel in „Central daily newspaper“ am 11. April 1958.

${ }^{293}$ Vgl. Hu Shi 1961 und 1962 (a), S. 39-41.

${ }^{294}$ Vgl. ders. (b), S. 5.

295 Vgl. Fu Li-yuh 2006 (a), S. 364.

2961957 wurde die Zeitschrift ,Wen-hsing “ (1957-1965) zur Vermittlung von Literatur und Kunst des Westens herausgegeben. Ab 1961 schrieb Li Ao, der mit der Lehre von Hu Shih sehr vertraut war und mit ihr übereinstimmte, Artikel. Vgl. Chen Cheng-jan 1985, S. 31f.
} 
„Wissenschaft“ und Technologie nach China zu verpflanzen und gleichzeitig zu hoffen, dass Werte und Normen davon nicht negativ beeinflusst würden. ${ }^{297}$ Daher forderte er die Ablehnung der chinesischen Tradition und eine radikale Verwestlichung, wenn Taiwan den Weg zum Aufbau eines modernen und mächtigen Staates gehen wolle. Die Folge war, dass die Zeitschrift ab 1965 von der KMT-Regierung verboten wurde.

\section{(2) Die Widerstände im Inneren}

Nach der fünfzigjährigen Trennung zwischen Taiwan und dem chinesischen Festland war eine breite Kluft zwischen zwei Gruppen entstanden, den Taiwanesen und den neu hinzugekommenen Chinesen, die auf sprachliche Unterschiede und historische Erfahrungen zurückgeht. Es gab nur wenige Chinesen in der neuen Regierung, die die taiwanesische oder die japanische Sprache beherrschten. Umgekehrt gab es kaum Taiwanesen, die des Hochchinesischen mächtig waren. Es fehlte folglich an Kommunikationsmöglichkeiten zwischen beiden Gruppen. ${ }^{298}$ Außerdem verloren zahlreiche Taiwanesen ihren Arbeitsplatz wegen mangelnder Sprachkenntnisse, zugleich wurde die japanische Sprache, in der sich die taiwanesischen Gelehrten auszudrücken pflegten, in der Öffentlichkeit verboten. ${ }^{299}$ Wegen dieser Umstellung erfuhren die Taiwanesen Diskriminierung und Unterdrückung. Am 28. Februar 1947 kam es zu einer Revolte, bei der Tausende, die gegen korrupte Regierungsmitglieder einen Teil der Truppe von Chiang Kai-shek - demonstrierten, ums Leben kamen. Unter den Toten befanden sich zum größten Teil Intellektuelle, die während der japanischen Besatzungszeit studiert hatten. Das Massaker nahm starken Einfluss auf die politische Struktur, denn ein großer Teil der Führungselite, der Beamten,

\footnotetext{
297 Vgl. Li Ao 1962 (a), S. 11 und ders. (b), S. 3-4.

298 Vgl. Cheng Tzu 1994 und Wang Chih-ping 2002, S. 66.

299 Vgl. ebd., S. 29f. und 54-57.
} 
Gelehrten und Kapitalisten, wurde dadurch beseitigt. ${ }^{300}$ In der Folge wurde die Herrschaft der vom chinesischen Festland zugewanderten Bevölkerung abgeschwächt und die Repräsentation der Taiwanesen in Parlamenten und Parteien stärker ihrem Anteil an der Bevölkerung angeglichen. Die massive Flüchtlingsfrage stellte große Herausforderungen an die taiwanesische Gesellschaftsordnung, ${ }^{301}$ zumal die KMT-Regierung eine Reihe von Förderungsmaßnahmen für Flüchtlinge und Soldaten durchgeführt hatte, um ihnen Privilegien und Wohlstand zu sichern. ${ }^{302}$

Die KMT-Regierung war anch dem Zweiten Weltkrieg einer direkten Drohung durch die chinesischen Kommunisten ausgesetzt. Um einen weiterführenden Bürgerkrieg vorzubereiten, wurden die „Vorläufigen Bestimmungen zur Mobilisierung für die Zeit der Niederschlagung der kommunistischen Rebellion“ (1948-1991) und das Notstandsgesetz (1949-1987) verhängt. Im Zeitraum von 1959 bis 1961 bzw. zur Zeit des Vietnam-Krieges plante Chiang Kai-shek einen Rückeroberungskrieg gegen das chinesische Festland. 1963 verwies jedoch US-Präsident Kennedy darauf, dass die USA gemäß Verteidigungspakt von 1954 nur im Verteidigungsfall zur Hilfe verpflichtet seien. Zusätzlich zu dieser Washingtoner Erklärung übte er Druck auf Taiwan aus. ${ }^{303}$ Dadurch wurde der Wunsch nach Rückeroberung Chinas langsam unmöglich. Nichtsdestotrotz predigte die KMT-Regierung nach wie vor den Kampf gegen den Kommunismus und sah die Wiedereroberung als oberstes Ziel des Staates an, um mit dem Bürgerkrieg, dem Kriegsrecht und zahlreichen Ausnahmegesetzen fortfahren zu können. ${ }^{304}$ Aufgrund der „Vorläufigen Bestimmungen“ sollten die

\footnotetext{
300 Vgl. Lai Tse-han 1947, S. 412; Wang Shiao-poa 1988; Wakabayashi Masahiro 1997, S. 74 und Günter Whittome 1991.

301 Vgl. Kindermann 1974, S. 15 und Li Hsiao-feng 1991.

302 Vgl. Lin Chiou-huang 2006; Chen Chi-ming 2000 und Chang Po-chung 1996.

303 Vgl. Lo Wen-hao 2005 und Peng Ta-nien 2005.

304 Die politischen Schlagworte in den 1950er-Jahren waren z.B. „Erstes Jahr für die Vorbereitung, zweites Jahr für die Rückeroberung, drittes Jahr für die Beseitigung der Kommunisten, im fünften Jahr
} 
Mandate der in den Jahren 1947/48 auf dem chinesischen Festland gewählten Abgeordneten der Nationalversammlung der fünf Staatsräte (Legislative, Exekutive und Justizgewalt, Prüfung und Kontrolle) ${ }^{305}$ bis zur „Befreiung“ der kommunistisch beherrschten Gebiete eingefroren werden. ${ }^{306}$ Das Kriegsrecht setzte zahlreiche verfassungsmäßige Grundrechte außer Kraft, in erster Linie die Pressefreiheit, das Postgeheimnis und die Rede- und Versammlungsfreiheit. Vor allem aber wurde die Gründung politischer Parteien verboten, die wichtige Elemente für eine demokratische Gesellschaft darstellen. Dadurch sollte jeglicher Einfluss bzw. jegliche Aktivität der Kommunisten auf Taiwan verhindert und schließlich die Alleinherrschaft weiterhin gesichert werden. ${ }^{307}$ Die Verhängung des Kriegsrechts wurde indes häufig infrage gestellt, und zwar von neuen politischen Gegnern der KMT, den vom chinesischen Festland emigrierten Liberalen, ${ }^{308}$ den Vertretern für die radikale Verwestlichung, ${ }^{309}$ sowie den Taiwanesen, die ins Exil gegangen waren und sich die Gründung eines von China unabhängigen Staates wünschten. ${ }^{310}$ Später, seit den 1960er-Jahren,

wird das Ziel erreicht”. In: Chiang Kai-shek 1950, S. 266.

305 Vgl. 5.5.1.2. in dieser Arbeit.

306 Vgl. Günter Schubert 1998, S. 207-209.

307 Zahlreiche taiwanesische Gelehrte und „verdächtige Kommunisten“ wurden von 1948-1987 umgebracht. Außerdem durften nur loyale KMT-Anhänger nach Taiwan übersiedeln. Ihre parteiinternen Gegner sollten auf dem Festland bleiben oder ins Ausland flüchten. Es war die sogenannte „Herrschaftszeit mit weißem Terror“. Vgl. Lan Po-chou 1993 und Tai Pao-tsun 2006, S. 297-305.

${ }_{308}$ Hu Shih, Lei Chen, Wang Shih-chieh u.a. versuchten mit der Zeitschrift „Free China“ den „liberalen und demokratischen“ Geist der „Bewegung des Vierten Mai 1919“ auf Taiwan fortzusetzen. $\mathrm{Zu}$ Beginn wurde ein für die KMT-Regierung positives Bild durch die Herausgabe dieser Zeitschrift gezeichnet, nämlich als eines Vertreters gegen den chinesischen Kommunismus in der internationalen Gesellschaft. Anschließend forderten diese emigrierten Liberalen nicht nur die Liberalisierung der Wirtschaft, sondern auch den Aufbau eines demokratisch-verfassungsgemäßen Politiksystems. Daher kritisierten sie auch die Maßnahmen der faschistischen Herrschaft Chiangs in unterschiedlichen Bereichen. Vgl. Chan Yoa-chi 2005, S. 30-41; Yen Shu-fang 1989; Su Rui-qiang 2003 und Hsueh Hua-yuan 1995, S. 127-160.

309 Vgl. 6.2.4.1. in dieser Arbeit.

${ }^{310}$ Ein Teil der taiwanesischen Gelehrten war nach dem „Aufstand am 28. Februar 1947“ ins Ausland geflohen. Mit der Bodenreform verloren die Großgrundbesitzer ihren Einfluss auf die Gesellschaft. Der Nachwuchs der Großgrundbesitzer flüchtete entweder ins Ausland, nach Japan, Europa und in die USA. In den 1960er-Jahren wurde von den emigrierten Taiwanesen wie Liao Wen-i (1920-1986), Koh Se-kai (1934-bis Gegenwart) u. a. sowie Nachkommen der Großgrundbesitzer wie Peng Ming-min (1923-bis Gegenwart) u.a. in Japan eine „Gesellschaft zum Streben für die Unabhängigkeit Taiwans“ errichtet, deren Einfluss weiter auf die emigrierten Taiwanesen in den USA und Europa wirkte und zur „Bewegung für die Unabhängigkeit Taiwans“ führte. Vgl. Tai Pao-tsu 2006, S. 306-322; Shih Ming-hsiung 1998 und Su Ching-hsuan 2008. 
veröffentlichten Dissidenten Artikel in Zeitschriften, etwa „Taiwan Political Review“ und „Formosa“.

Diese unterdrückte zwar die Meinungen, welche ihrem Kurs entgegenstanden mithilfe von Pressezensur und Verleumdung, jedoch erkannte sie auch, dass sie eine neue Basis für die Legitimierung ihrer Herrschaft über Taiwan schaffen musste, da sie den Krieg zur Rückeroberung des chinesischen Festlands nicht weiter führen konnte. Gleichzeitig forcierte sie die Industrialisierung durch Verstärkung der naturwissenschaftlichen Forschung und Ausbildung sowie die freie Marktwirtschaft. Die in China mobilisierte „Kulturrevolution“ (1966-1976), eine radikale Sozialbewegung gegen die chinesische Tradition, wurde von Chiang als Gelegenheit zur Lösung seiner politischen und ideologischen Krise genommen. Er rief im Gegenzug zu einer neuen Auslegung der „Drei Prinzipien des Volkes“ und zum Aufbau eines kapitalistischen Landes in Einklang mit dem Konfuzianismus auf.

\subsubsection{Chiangs Forderung nach „Ethik, Demokratie und Wissenschaft“"}

Chiang, der zu Beginn seiner Ankunft auf Taiwan die „Drei Prinzipien“ in populärer Weise umformuliert und „Demokratie“ und „Wissenschaft“ als zwei wesentliche Forderungen der „Bewegung des Vierten Mai 1919“ in die Staatslehre Suns integriert hatte, kritisierte andererseits, dass die „Bewegung zur Kulturerneuerung“ auf einer fremden Lehre, entweder dem westlichen Liberalismus und Pragmatismus oder dem Marxismus, basierte. Damit sei die Überlieferung der eigenen Kulturtradition gefährdet, sie werde als rückschrittlich und als „feudale Erbschaft“ betrachtet. Die Folge davon sei, dass die Jugendlichen den Unterschied zwischen der KMTRegierung, welche die chinesische Kultur und Tradition zu bewahren suche, und dem 
kommunistischen Regime Chinas, das die eigene Kultur und Tradition zerstöre und sich auf ein fremdes Land, Russland, richte, nicht erkennen könnten. Dies werde von den chinesischen Kommunisten zur Rebellion gegen die KMT-Herrschaft genutzt. ${ }^{311}$ Die Jugendlichen hätten ihre geistige Verteidigung gegen den Kommunismus verloren, und so sei China von den Kommunisten erobert worden. Seitdem Chiang seine Herrschaft über Taiwan war, forderte Chiang daher, neben „Demokratie“ und „Wissenschaft“ auch „Ethik“ zu einem erstrebenswerten Prinzip beim Aufbau des Landes zu erheben und mit dem „Prinzip des Volkstums“ gleichzusetzen, Demokratie mit „Prinzip der Volksrechte“ und Wissenschaft mit dem „Prinzip des Volkswohls“. ${ }^{312}$ Mit dieser Auslegung der „Drei Prinzipien des Volkes“ sollte eine neue Orientierung im Kampf gegen die chinesischen Kommunisten einhergehen, welche die KMT-Regierung in Taiwan rechtfertigte.

Anlässlich der Einweihung der Erinnerungsstätte Chung-shan ${ }^{313}$ im Jahr 1966 hielt Chiang eine Rede, in der er die „Drei Prinzipien des Volkes“ mithilfe konfuzianischer Ansätze auslegte, um zu zeigen, dass die Staatsutopie von Konfuzius ${ }^{314}$ und Suns „große Gemeinsamkeit der Welt“ durch Einführung der Konzepte von „Ethik, Demokratie und Wissenschaft" realisiert werden könnten. ${ }^{315}$ Daran forderte Chiang nun die Loyalität gegenüber der KMT-Regierung und dem Vaterland, welche ein hohes Maß an Gehorsam und Pflichtbewusstsein verlangten. Dies entspreche der konfuzianischen Vorstellung, den Herrscher als Vaterfigur und das Volk als seine Familie zu sehen, diesem Familienoberhaupt sei Respekt entgegenzubringen und seine Worte zu befolgen.

\footnotetext{
311 Vgl. Chiang Kai-shek 1952 (d), S. 162-164.

312 Vgl. ders. 1951 (b), S. 205-213.

313 Chung-shan war der andere Name von Sun Yat-sen.

$314 \mathrm{Vgl}$. 5.5.1.3. in dieser Arbeit.

315 Vgl. Chiang Kai-shek 1966 S.270-271.
} 
Dieses Modell des „Familismus“6316 wurde in der Folge auch auf Wirtschaft und Gesellschaft übertragen und einerseits der Regierung das Recht auf staatliche Eingriffe zugestanden und andererseits eine familiale Gesellschafts- und Unternehmensstruktur geprägt. Diese kulturellen Gegebenheiten wurden damit zur Grundlage der starken gesellschaftlichen Kohärenz und des ausgeprägten Kollektivbewusstseins, wodurch die während des Modernisierungsprozesses entstandene Disharmonie der sozialen Verhältnisse wie Entfremdung, Inflation, Arbeitslosigkeit und ungleiche Verteilung der Güter und des gesellschaftlichen Reichtums überwunden wurden.

Als zweite Grundlage der chinesischen Kultur proklamierte Chiang die Demokratie, denn „Bevölkerung ist etwas Wertvolles, und die Bevölkerung ist die Grundlage eines Landes. Wenn die Bevölkerung gut versorgt wird, dann herrscht Frieden. Deswegen versucht ein fähiger, weiser und erhabener Herrscher, im Inneren die Tüchtigsten und Fähigsten als Führungskräfte auszuwählen; zugleich spricht er die Wahrheit und pflegt die Eintracht.

Als Erläuterung seiner eigenen ununterbrochenen Herrschaft auf Taiwan verkündete Chiang nun seine These von der Legitimation einer Herrschaft auf Basis des „Erbes der chinesischen Kultur“. In der Tat gab es in der chinesischen Tradition keine Demokratie im westlichen Sinne. Zur Verstärkung seiner Aussagen - welche bis in die jüngste Gegenwart der chinesischen Rhetorik entsprechen - zitierte Chiang die Grundlagenwerke der chinesischen Klassik, darunter „Das Buch der Urkunde“, „Das Buch der Riten“ und „Das Buch von Mitte und Maß“, um an das Ideal eines Herrschers im Altertum anzuschließen. Ein „fähiger, weiser und erhabener 316 zum „Familismus“ siehe Wen Chung-i 1988, S. 49-84 und Yang Mao-chun 1988. 
Herrscher" stelle die Meinung und das Wohl der Bevölkerung immer in den Vordergrund und bemühe sich um Ordnung und Frieden sowohl im Innern als auch nach außen. Mithilfe dieser Prinzipien gewinne er die Fürsprache des Volkes. Chiang erklärte Sun Yat-sen zum Nachfolger der chinesischen Klassiker, Sun beerbe die chinesischen Kaiser und Weisen des Altertums Yao, Shun, Yu, Tang, Wen, Wu, Chou-kung und Konfuzius. Chiang selbst sei der wahre Nachfolger Suns und strebe danach, dessen Programme für den Aufbau des modernen Chinas zu realisieren. Damit suchte sich Chiang von Mao Tse-tungs Regime auf dem chinesischen Festland abzugrenzen, ${ }^{317}$ um als „,ähiger, weiser und erhabener Herrscher“ seine Macht in Taiwan abzusichern, während China unter Herrschaft der Kommunisten in einen chaotischen und rückständigen Zustand verfalle, indem sie die Bevölkerung unterdrücken und die zuvor existierende Marktwirtschaft in eine sozialistisch orientierte Planwirtschaft umwandelten. Durch die Kulturrevolution werde der „Klassenkampf“ gegen vermutete innere Feinde geführt, doch dieser richte sich zunächst gegen die eigene Kultur, gegen Sitten, Gewohnheiten und Denkweisen und damit auch gegen deren Träger, vor allem die Gebildeten und Gelehrten.

Im Gegensatz dazu sei die KMT-Regierung ein Bewahrer der chinesischen Kultur. Das Leben im „Free China“ - Chiang meint damit Taiwan - sei ein Paradies mit Demokratie, Fortschritt und Wohlstand, das Leben auf dem Festland gleiche dagegen einer Hölle. Die später ins Leben gerufene „Bewegung für die Bewahrung und Restauration der chinesischen Kultur“ verhieß Chiang als Befreier von den Leiden Chinas, bedingt durch die Herrschaft der Kommunisten, er sei das Heil der chinesischen Nation und großartigster Mensch der Welt. ${ }^{318}$

\footnotetext{
317 Vgl. John Makeham 2008, S. 192-207.

318 Vgl. Tai Pao-tsun 2006, S. 294f.
} 
Als dritte Grundlage der chinesischen Kultur stellte Chiang in seiner Rede die Wissenschaft vor; sie sei ein Weg, der dazu führen solle, dass „der Staatsführer seine Persönlichkeit und Integrität pflegt, damit er als Vorbild des Landes wirken kann. Dann hätten alle Dinge den besten Nutzen und dienten den Notwendigkeiten sowie der Bereicherung des Lebens und des Wohls der Bevölkerung.“ Auch Konfuzius habe gefordert, dass „die Politik eines Landes dafür sorgen soll, dass die Bevölkerung zu einem reichen und langen Leben gebracht wird.“ Der Weg dahin sei eine „ausgeglichene und gleichmäßige Verteilung der Güter, denn dann gibt es keine Armen. Wenn die Bevölkerung eines Landes mit anderen in Harmonie lebt, dann fehlt es ihr an nichts. Wenn alle Menschen friedlich miteinander umgehen, dann gibt es keine soziale Krisen. “6319

Chiang äußerte, dass naturwissenschaftliche Forschung in China nichts Neues sei, jedoch sei diese Tradition von den Gelehrten im Laufe der Zeit vernachlässigt worden; nun sollten sie sie wieder aufnehmen. Chiang lehnte Einstellungen wie „Autonomie der Forschung“ oder „Wertfreiheit der Wissenschaften“ ab, wie sie von liberalen Wissenschaftlern in dieser Zeit propagiert wurden. Auf Grundlage der konfuzianischen Lehre sei das entscheidende Ziel der Forschung die Befriedigung des Bedarfs und die Verbesserung der Lebensqualität der Bevölkerung in allen Bereichen wie Essen, Kleiden, Wohnen, Verkehr, Erziehung und Unterhaltung etc. Daher sollten künftig die Naturwissenschaften im Dienst der produktiven und wirtschaftlichen Entwicklung stehen. Nach Suns „Prinzip des Volkswohls“ sollte der im Laufe der Industrialisierung und Modernisierung gewachsene Reichtum gemäß den Prinzipien von „gleichmäßiger Verteilung des Bodens und Wohlstands“ und „Beschränkung des Privatkapitals“ aufgeteilt werden. Zugleich sollten die konfuzianischen Tugenden wie

319 Zit. nach Chiang Kai-shek 1966 S.270f.. Eigene Übersetzung. 
Loyalität gegenüber der Obrigkeit und Verantwortung der Gemeinschaft bzw. den Untertanen gegenüber auch auf das Verhältnis zwischen Arbeitnehmern und Arbeitgebern übertragen werden, um soziale Konflikte zu vermeiden.

Mit seiner Auslegung versuchte Chiang, dem Modell der taiwanesischen Modernisierung eine Basis zu geben und einen „konfuzianischen Kapitalismus“ zu gestalten. Er vertrat damit die Meinung der Neokonfuzianisten, ${ }^{320}$ welche die Einführung der wissenschaftlichen Kenntnisse den traditionellen Werte und Normen unterordnen wollten, um ihre soziale Sprengkraft zu entschärfen. Dadurch sollte sie für die bestehende Ordnung nutzbar gemacht werden.

\subsubsection{Inhalte der „Bewegung für die Bewahrung und Restauration der chinesischen Kultur"}

Nach der Rede Chiang Kai-sheks riefen ca. 15.000 KMT-Anhänger zur „Bewegung für die Bewahrung und Restauration der chinesischen Kultur“" auf, unter ihnen auch Sun Ke (1891-1973), Enkel von Sun Yat-sen, Chen Li-fu (1900-2001) ${ }^{321}$ und Kung Te-cheng (1920-2008), Nachkomme in der 77. Generation von Konfuzius. ${ }^{322} 1967$ wurde das „Komitee für die Förderung der Bewegung zur Bewahrung und Restauration der chinesischen Kultur“ gegründet. Dessen Aufgabe bestand auch in

\footnotetext{
${ }^{320}$ Nach der „Vierten-Mai-Bewegung 1919“ bemühten sich Kulturkonservative wie Chang Chun-mai, Tang Chun-i u.a. um einen Weg zum Aufbau des modernen Chinas aus der Tradition und zugleich den Neokonfuzianismus mithilfe westlicher Philosophie neu auszulegen. Einige wichtige Vertreter des Kulturkonservativismus wie Mou Tsung-san, Hsu Fu-kuan, Hsiung Shih-li, Fang Tung-mei und Chien $\mathrm{Mu}$ übersiedelten nach 1949 nach Hongkong und Taiwan und trugen zur Gestaltung der konfuzianischen Modernisierungsthese bei. Vgl. Liu Shu-hsien 1997, S. 54-60.

${ }^{321}$ Chen Li-fu war Mitglied des KMT-Komitees und stand Chiang Kai-shek nahe. Die theoretische Grundlage der „Bewegung Neues Leben” wurde von Chen gestaltet, 1938-1944 war er Bildungsminister. Während der „Bewegung zur Restauration der chinesischen Kultur“ auf Taiwan unterstützte er die Untersuchung und Verbesserung der chinesischen Medizin mit wissenschaftlichen Methoden, damit die chinesische Medizin als Wissenschaftsbereich anerkannt werden konnte. Zugleich war er zuständig für die Edition der chinesischen Wissenschaft und Kultur (1967-2001) und amtierte als Präsident der „Confucius society“.

${ }^{322}$ Vgl. Lin Gou-shian 2001, S. 48-53 und Yang Rong-cong 1992, S. 46-48
} 
einer „kulturellen Kriegsführung“ gegen die chinesischen Kommunisten. ${ }^{323}$ Folgende Maßnahmen sollten helfen, die Kultur in den Vordergrund zu rücken:

(1)Überarbeitung der Schulbücher;

(2)Herausgabe und Neukommentierung literarischer und philosophischer Klassiker;

(3)eine Kampagne zur Schaffung neuer Werke in Kunst und Literatur im Geist von Ethik, Demokratie und Wissenschaft;

(4)Bau von Sportanlagen, Theatern, Musikhallen, Kunsthallen und Museen;

(5)verstärkte Einbeziehung der Massenmedien, um die Sittlichkeit in der Bevölkerung voranzubringen;

(6)Wiederbelebung der konfuzianischen Tugenden;

(7)stärkere Hervorhebung nationaler Eigenschaften im Dienste der Tourismusindustrie;

(8)Bewahrung der historischen Kulturgüter und Schätze;

(9)Förderung von Bildung und Forschung in Bezug auf die chinesische Kultur, auch bei Auslandschinesen und ausländischen Forschungsinstituten;

(10)Förderung von Investitionen in privater Trägerschaft für kulturelle und erzieherische Einrichtungen. $^{324}$

Das Lernen konfuzianischer Klassiker, traditioneller Kunst, Musik, Kampfkunst und Kalligraphie wurde sowohl in der Schule als auch in der Gesellschaft aktiv gefördert und die Kulturrenaissance durch eine Reihe von anderen Aktivitäten, Ausstellungen und Veranstaltungen, begleitet, etwa der Eröffnung des „Nationalen Palastmuseums“، ${ }^{325}$ der Neubelebung traditioneller Architekturformen, der Befürwortung

\footnotetext{
23 Vgl. Shih Chih-hui 1995, S. 22-24.

324 Vgl. Chang Shu-lun 1998, S. 211.

325 Die Kunstsammlung beinhaltete etwa 620.000 Exponate die, von Kaisern der Ming- und ChingDynastie gesammelt, von Chiangs Regime nach Taiwan mitgebracht worden waren. Alle diese Kulturschätze wurden seit 1965 im Nationalen Palastmuseum in Taipei ausgestellt.
} 
von Volksreligionen sowie der Errichtung von Forschungsinstituten über die „Drei Prinzipien des Volkes“. ${ }^{326}$ Schulen, Straßen und öffentliche Gebäude wurden nach konfuzianischen Tugenden, nach Sun Yat-sen und Chiang Kai-shek sowie nach Provinzen und Städten auf dem chinesischen Festland benannt, in allen öffentlichen Einrichtungen hingen Bilder oder standen Statuen von Sun Yat-sen, Chiang Kai-shek und Konfuzius. Zudem erstellte das „Komitee für die Anleitung zum alltäglichen Leben der Bevölkerung“ ein „Handbuch für bürgerliche Instruktionen“ (1967) mit 99 Regeln auf Basis der konfuzianischen Tugenden für Essen, Kleidung, Wohnen, Verkehr, Erziehung und Unterhaltung. Ferner regelte das „Handbuch für die bürgerliche Konvention“ (1970) Benehmen, Kleidung und Umgang in der Öffentlichkeit sowie den Ablauf von Veranstaltungen, Hochzeiten, Beerdigungen, Feiern etc., wodurch das richtige Benehmen als moderne Chinesen in einem modernen Staat mit chinesisch traditioneller Sittlichkeit und Höflichkeit verbunden werden sollte. $^{327}$

\subsubsection{Der Einfluss der „Bewegung für die Bewahrung und Restauration der chinesischen Kultur" auf die schulische Erziehung}

Nachdem ab 1952 alle Lehrbücher gemäß den „Drei Prinzipien des Volkes“ neu editiert worden waren, sollte nun die Gestaltung der Schulerziehung besondere Rücksicht auf die „Bildung des Volksgeistes“ und die Moralerziehung nehmen und mit der „Bewegung für die Bewahrung und Restauration der chinesischen Kultur“ koordiniert werden. ${ }^{328}$ Die Fächer Chinesisch, Geschichte, Geographie sollten die Aufgabe der „Bildung des Volksgeistes“ übernehmen. Zum Beispiel wurde hier die Mongolei zum Hoheitsgebiet Chinas gezählt, obwohl sie seit 1953 ein

\footnotetext{
326 Vgl. Oskar Weggel 1991, S. 155-163.

327 Vgl. Huang Huang-ping and Chiu Lian-Hwang 1991, S.376 und Richard Wilson 1970.

328 Vgl. Bildungsministerium (Hrsg.) 1974, S. 440.
} 
unabhängiger Staat war. Alle Änderungen durch die VR China wurden von der KMT nicht anerkannt, wodurch eine eigene Geographie entstand. ${ }^{329}$ Taiwan wurde im Lehrbuch als Teil des Chinas propagiert, jedoch als ein peripherales und kulturrückständiges Gebiet Chinas bezeichnet.

Während andere Fächer, wie „Alltag und Ethik“ in der Grundschule, „Staatsbürgerkunde und Tugenden“‘330 sowie Pfadfindererziehung in der Junior High School und „Staatsbürgerkunde und Tugenden“, „Drei Prinzipien des Volkes“ und „militärische Instruktion“ in der Sekundarstufe II, ${ }^{331}$ in direkter Verbindung mit der Parteiideologie, der Verehrung, ja Vergötterung der Staatsführer, Chiang Kai-shek und sein Sohn Chiang Ching-Kao sowie der Geisteshaltung des Antikommunismus, der Bedeutung der chinesischen Kultur in der Welt, den „Drei Prinzipien des Volkes“ gewidmet waren. Zudem sollte Taiwan zum Vorbild der „Drei Prinzipien des Volkes“ für die anderen Provinzen Chinas aufgebaut werden. ${ }^{332}$ Dort wurde auch die Notwendigkeit der Rückeroberung des Festlandes und die Befreiung der Landsleute in China proklamiert und gleichzeitig die konfuzianischen Tugenden mit militärischen und patriotischen Ideen von Etikette, Gehorsam, Gemeinschaftsgeist, Vaterlandsliebe und Loyalität gegenüber den politischen Führern sowie Kampfeswillen verbunden.

Das alltägliche Leben der Schüler in Bezug auf Benehmen, Essen, Kleidung sowie konkrete Grundhaltungen des Lebens wie Sauberkeit, Disziplin und traditionelle sittliche Ordnung sollten kultiviert werden. Dazu wurde bei jeder Gelegenheit, etwa

\footnotetext{
${ }^{329}$ Vgl. Wang Wen-lung 2008, S. 201-251.

330 Die Analyse der Lehrinhalte findet man in ,Zur aktuellen Methodik der Moralerziehung in Taiwan: der Sinn und die Problematik der Realisierung des ,Werterziehungsansatzes“ in den Lehrbuchreihen des Faches Civics, Staatsbürgerkunde für den Primarbereich, und die Anwendungsmöglichkeit dieses Modells für den Sekundarbereich“, vorgelegt von Lan Yu-su 1996; Kao Chien-her 2006 und Shih Chisheng 1993.

331 Vgl. Lin Gou-shian 2001, S.74f.

332 Vgl. Shih Chi-sheng 1993, S.85f.
} 
auf Schulversammlungen, der tiefere Sinn der traditionellen chinesischen Handlungsnormen und Tugenden erklärt, ${ }^{333}$ welche zugleich zur Überwachung der Schüler und als Kriterien für die Bewertung der Persönlichkeitsentwicklung verwendet wurden: Im Schulzeugnis gab es eine sogenannte „Persönlichkeitsbewertung“. 334

Daneben wurde das Studium der chinesischen Klassiker sowie der Geschichte und Geographie Chinas verstärkt. Mit der 1953 vom Bildungsministerium edierten Reihe chinesischer Klassiker, den „Lektüren für die Basis der chinesischen Kultur“, war die Lektüreauswahl der Schulen vorbestimmt. Auf Betreiben der „Bewegung für die Bewahrung und Restauration der chinesischen Kultur" wurden nun vier Klassiker des Konfuzianismus mit neuem Kommentar von Chen Li-fu alsVerbindung zwischen der Lehre von Konfuzius mit den „Drei Prinzipien des Volkes“ in der Schule eingesetzt und die Klassiker sowie Kampfkunst, Kunst und Musik einbezogen. ${ }^{335}$ Zugleich sollten Kenntnisse über internationale Bedingungen sowie Sitten für moderne Staatsbürger vermittelt werden.

In den Hochschulen wurden die Fächer „Chinesisch“, „Moderne Geschichte Chinas“, „Drei Prinzipien des Volkes“ und die „Lehre vom Landesvater“ sowie das „Studium der Fragen des chinesischen Festlandes“ zu Pflichtfächern. Lehrinhalte waren Werk und Vermächtnis von Sun Yat-sen, Taten, Reden und Aufsätze von Chiang Kai-shek, die „Tyrannei der chinesischen Kommunisten“ und internationale Beziehungen. ${ }^{336}$

\footnotetext{
333 Vgl. Huang Chung 1980, S. 167-176.

334 Im Schulzeugnis wurden vier Bereiche begutachtet: Kognitives, Persönliches (die Leistung im Fach Staatsbürgerkunde sowie Benehmen), Sportliches sowie Gemeinschaftliches (Pfadfindererziehung und außerschulische Aktivitäten). Vgl. Bildungsministerium (Hrsg.) 1974, S. 396-399.

${ }^{335}$ Vgl. Huang Chun-chieh 1992, S. 228f. und ders. 2001, S. 283-89. Vgl. Bildungsministerium (Hrsg.) 1974, S. 440f.

336 Vgl. Chang An-jan 1987, S. 262-276.
} 
Chiang kritisierte solche Lehrbücher vor allem der geistes- und sozialwissenschaftlichen Fächer, die direkt aus den westlichen Ländern übernommen worden waren, und forderte zu einer Neubearbeitung auf Basis der „Drei Prinzipien des Volkes“ auf. ${ }^{337} \mathrm{Zu}$ diesem Zwecke wurden Institute für das Studium der „Drei Prinzipien des Volkes“ an der „National Taiwan Normal University“ (1967), der „National Taiwan University“ (1974), der „National Chen Chi University“ (1974), der Wen Hua Fachhochschule (1962), der Militärfachhochschule (1968) und an der „Academia Sinica“ (1981) errichtet. ${ }^{338}$

Zusammen mit der „Bewegung für die Bewahrung und Restauration der chinesischen Kultur“ wurde nun mit der „Bewegung für die Verwendung der Nationalsprache“ Chinesisch noch radikaler als zuvor durchgesetzt ${ }^{339}$ und mit dieser Dominanz der chinesischen Kultur die Identität der Taiwanesen unterdrückt. ${ }^{340}$ In allen Anstalten und Schulen, aber auch in der Öffentlichkeit sollte nur Hochchinesisch gesprochen werden. Zuwiderhandlung der Lehrer und Schüler wurde unter Strafe gestellt und chinesische Tradition und Kultur wie die japanische Kultur unter der japanischen Besatzung politisiert und zur Assimilierung und zur Schaffung einer einheitlichen „Nation“ instrumentalisiert. ${ }^{341}$ Die Verwendung des Japanischen sowie des Taiwanesischen - der einheimischen Sprache auf Taiwan nach dem Zweiten Weltkrieg - galt für die KMT als Hindernis bei der Ausbildung zu einem chinesischen Nationalbewusstsein und der Integration der Taiwanesen. So entfremdeten die sinisierten Lehrinhalte die Schüler von ihrer Lebenswelt. Hinzu kam, dass China

\footnotetext{
337 Vgl. Chiang Kai-shek 1968, S. 429f.

338 Vgl. Chang An-jan 1987, S. 58-60.

339 Vgl. Chun Allen 1996, S. 126-147.

340 Vgl. Lin Gou-shian 2001, S. 75-78.

${ }^{341}$ Z. B. mussten die Schüler in der Schule ein Schild „Taiwanesisch sprechen heißt, es mangelt an Vaterlandsliebe" umhängen, oder sie wurden mit Bußgeld, Abzügen in der Note für Betragen, Arbeitsdienst oder Schlägen bestraft, wenn sie Taiwanesisch sprachen. Vgl. Chen Bo-chang 1991, S. 78 ; Chen Mei-ju 1996, S. 128f. und Alan M. Wachman 1994, S. 53.
} 
durch das Kontaktverbot mit dem chinesischen Festland für viele Schüler ein fremdes und fernes Land war. Lehrinhalte mit Bezug auf Taiwan wurden erst nach Aufhebung des Kriegsrechts in den 1990er-Jahren langsam wieder einbezogen. ${ }^{342}$

Insgesamt lieferte die „Bewegung für die Bewahrung und Restauration der chinesischen Kultur“ eine Grundlage für Aufbau eines modernen Chinas auf Taiwan, durch die Loyalität der Jugendlichen den Staat bzw. das KMT-Regime gegenüber zu stärken, die Wissenschaft zum Prinzip der neuen Erziehung zu erheben, die Sparsamkeit und Rationalität zum Beitrag der wirtschaftlichen Modernisierung aufzuziehen, zugleichzeitig die Werte und Normen als moderne Staatsbürger unter der Bevölkerung bzw. Jugendlichen zu verbreiten.

6.3. Pläne für die Gestaltung der beruflichen Ausbildung sowie der naturwissenschaftlichen und technischen Forschung und Bildung

Neben der militärischen Erziehung und der „Bildung des Volksgeists“ erstellte das KMT-Regime auch einen Reihen der Pläne für die Gestaltung der beruflichen Ausbildung sowie der naturwissenschaftlichen und technischen Forschung und Bildung. In diesem Abschnitt untersuche ich die entsprechnde Bildungspolitik des KMT-Regime.

\subsubsection{Die Debatte über berufliche Ausbildung sowie naturwissenschaftliche und} technische Forschung und Bildung innerhalb des KMT

Nach Betrachtung des Pädagogen Huang Jen-pei (1878-1965) ${ }^{343}$ litt die berufliche Ausbildung nach Gründung der Republik China unter folgenden Schwierigkeiten:

\footnotetext{
342 Vgl. Tsai Ming-hsien 2009, S. 82-152.

343 Huang Jen-pei (1878-1965) trug stark zur Einführung der modernen Berufsausbildung und zum Aufbau des beruflichen Bildungssystems auf Taiwan bei. Vgl. Tsao Chang-jen und Lin Chung-yao 2003, S. 38-41.
} 
Erstens entsprach die Gestaltung der Fächer nicht der Arbeitswelt zu jener Zeit, und zweitens waren die Lehrgänge aufgrund direkter Übernahme des Stoffs aus fremden Ländern zu theoretisch gestaltet, und es gab keine Werkstätten oder Bauernhöfe zur praktischen Veranschaulichung. ${ }^{344} \mathrm{Um}$ das chinesische Bildungswesen effektiv zu reformieren, hatte die KMT-Regierung 1931 beim Völkerbund einen Antrag auf pädagogische Beratung gestellt. Das „International Committee on Intellectual Cooperation“, die zuständige Unterorganisation des Völkerbundes, entsandte daraufhin die „League of Nations Mission of Educational Experts“ nach China. ${ }^{345}$ Nach dreimonatigem Besuch stellten die Berater ihren Bericht „Die Reorganisation des chinesischen Bildungswesens“ vor. Die Reformvorschläge orientierten sich an den Richtlinien des provisorischen Plans für die wirtschaftlich-soziale Entwicklung des Landes und bezogen sich vorrangig auf die Schulorganisation sowie die Hochschulbildung und den naturwissenschaftlichen und technischen Unterricht. ${ }^{346}$ Der Aufbau des staatlichen Bildungswesens sollte mit anderen Teilsystemen der Gesellschaft koordiniert werden und für Arbeitskräfte aus allen sozialen Schichten durch differenzierte Bildungszweige und Lehrgänge gültig sein. Dazu sollte die chinesische Regierung eine zielgerichtete und planmäßige Erweiterung der Bildungsplätze in Koordination mit dem realen Bedarf an Arbeitskräften gemäß den Wirtschaftsplänen anstreben. Immerhin gewann dieser auf empirischen Untersuchungsbelegen zur schulischen Realität Chinas mit dem Kommentar von pädagogischen Fachberatern aus dem Völkerbund basierende Bericht der kulturkonservativen und nationalistischen Nankinger KMT-Regierung große

\footnotetext{
${ }^{344}$ Vgl. Chiu Hsiu-hsiang 2005, S. 151.

345 Die Mitglieder waren Carl H. Becker, Professor an der Universität Berlin und ehemaliger Preußischer Kulturminister (1927-1931) als Vorsitzender, Prof. M. Falski, Direktor der Abteilung der Elementaren Bildung im Polnischen Kulturministerium, Prof. P. Langevin, Collège de France, und Prof. R.H. Tawney von der „London School of Economics and Political Science“. Vgl. Die Bildungskommission des Völkerbunds 1963, S.1-2.

${ }^{346}$ Vgl. Chang Li 1986, S. 281-314 und Hu Chang-chih 1987, S. 37-50; Die Bildungskommission des Völkerbunds 1963 und Ernst Neugebauer 1971.
} 
Aufmerksamkeit, denn damit die Legitimation der KMT-Regierung für Aufbau eines einheitlichen, national bezogenen und wirtschaftsentwicklungsorientierten Bildungswesen verstärkte. ${ }^{347}$

Nach seinem Rückzug nach Taiwan charakterisierte Chiang den Versuch folgendermaßen: „Die wesentlichen Ursachen für unser Misslingen lagen in der Erziehung und Kultur ... Abgesehen von der moralischen Erziehung redeten wir nur über die kognitive Erziehung. Wenn wir eine Gesamtplanung und eine ausgeglichene Durchführung der Pläne etwa für Produktionserziehung, Berufsausbildung, naturwissenschaftliche und technische Erziehung u. a. gehabt hätten, wäre unser Land nicht in eine solch miserable Lage geraten und nicht von solch einem Missgeschick getroffen worden.“348 In den „Zwei Aufsätzen über $\mathrm{Yu}$ und Le (Erziehung und Unterhaltung des Volkes) zur Ergänzung des ,Prinzips des Volkswohls““ (1953) zeigte Chiang drei wesentliche Schwächen in der schulischen Erziehung in den 1930er- und 40er-Jahren auf dem chinesischen Festland auf:

(1) „Sheng-hsueh chu-i“(升學主義, zu Deutsch: „blinden Bildungseifer“): Die Unterrichtsgestaltung der Grund- und Mittelschule sei nur als Vorbereitung für das Universitätsstudium bzw. zum Bestehen der Übergangsprüfung angesehen worden. Jedoch sei auf die Vorbereitung auf das reale Leben, die moralische Entwicklung und die künftige Erwerbstätigkeit kaum Rücksicht genommen worden.

(2) Formalismus: Mit Einführung des modernen Bildungssystems hätten die Bildungsanstalten zwar zur Verleihung des formellen Diploms und Zertifikats beigetragen, jedoch sei die Gestaltung des Unterrichts inhaltsleer gewesen. Sowohl die Einrichtungen als auch die Lehrkräfte seien schlecht gewesen, und die Studenten

\footnotetext{
347 Vgl. Chang Li 1986, S. 281-314.

348 Zit. nach Chiang Kai-shek 1951 (b), S. 208. Eigene Übersetzung.
} 
hätten weder in der Forschung noch in beruflicher Kompetenz ausgebildet werden können.

(3) Isolationismus: Die Gestaltung der naturwissenschaftlichen und technischen Bildung in Schule und Hochschule sei zu theoretisch und für die industrielle Entwicklung des Landes zu wenig praktikabel gewesen. Es sei notwendig, dass die Schüler und Studenten in die Fabrik oder auf einen Bauernhof gingen, und dort ein Praktikum abzuleisten, damit sie lernen, ihre Kenntnisse im realen Leben anzuwenden. ${ }^{349}$

Die Berater der Bildungskommission des Völkerbunds erwähnten in ihrem Bericht, dass viele Gelehrte nach wie vor die traditionelle konfuzianische Erziehung verfolgten. Die modernen Hochschulen würden von der Gesellschaft als neue Institutionen zur Auswahl und Ausbildung künftiger Beamten und analog dazu der Besuch der modernen Schule als Vorbereitung auf die kaiserliche Beamtenprüfung angesehen. Daher seien die Inhalte der schulischen Erziehung noch immer sehr literarisch ausgerichtet, das Bildungswesen lege seine Schwerpunkte auf die allgemeinbildende Mittelschule sowie die Geistes- und Sozialwissenschaften in den Hochschulen, während die berufliche Ausbildung und die Naturwissenschaften sowie die Technik wenig berücksichtigt würden. ${ }^{350}$ Zudem gäbe es eine große Diskrepanz zwischen schulischer Erziehung und realem Leben. Da die Bildungsinhalte häufig direkt aus Fremdländern übernommen worden seien, hätten die Jugendlichen überzogene Erwartungen an das Leben, die nicht $\mathrm{zu}$ verwirklichen seien, was $\mathrm{zu}$ Unzufriedenheit führe. Diese Analyse klärte die KMT-Politiker auf, dass zwar die Kommunisten die Studenten und Schüler zu Streiks und Revolten aufgestachelt

\footnotetext{
349 Vgl. Chiang Kai-shek 1953, S. 221-223.

350 Vgl. Die Bildungskommission des Völkerbunds 1963, S. 165.
} 
hätten, aber die disharmonische Gestaltung des Schulsystems habe zur Unzufriedenheit beigetragen.

Dies alles wurde von Chiang als Hindernis beim Aufbau eines modernen chinesischen Bildungswesens im Dienst der Wirtschaftsentwicklung und Industrialisierung betrachtet. Er setzte seine Kritik fort und warnte: „Je mehr Junior und Senior High Schools errichtet werden, desto mehr ,Parasiten in der Gesellschaft‘ werden erzogen“, da die Schüler keine Kompetenzen zur Steigerung der Produktion und der sozialen Entwicklung entwickelten. ${ }^{351}$ Schulische Bildung diene indes keinesfalls nur den Zweck der Aufklärung, vielmehr stelle Allgemeinbildung bzw. bürgerliche Erziehung, wie sie von der „Bewegung des Vierten Mai 1919“ und in den 1920er-Jahre gefordert und propagiert wurde, einen unrealistischen Luxus dar. ${ }^{352}$ Zudem stehe das Streben nach weiterführender Bildung zum Erwerb eines Bildungstitels als individuelles Streben nach eigenem sozialem Aufstieg im Widerspruch zu den Erziehungskonzepten der KMT-Regierung, welche die Erziehung „im Dienst der Rettung bzw. der Weiterexistenz unseres Vaterlandes“،353 sah.

Mit diesen Ansätzen wies Chiang Kai-shek den Weg für die künftige Entwicklung des taiwanesischen Bildungswesens, die Förderung der praktischen Ausbildung als „Bildung für alle“, um die Produktionstechniken durch naturwissenschaftliche und technische Forschung und Ausbildung zu verbessern und die Entwicklung des Landes zu unterstützen. ${ }^{354}$ Dadurch könne die ideale Gesellschaft nach dem „Prinzip des

\footnotetext{
351 Vgl. Chiang Kai-shek 1951 (c), S. $269 f$.

352 Vgl. Chiang Kai-shek 1951 (c), S. 267.

353 Vgl. ders. (b) S.211.

354 Vgl. ebd., S. 156-158 und 160-165.
} 
Volkswohls“ verwirklicht werden, indem die Fähigkeiten und Begabungen der gesamten Bevölkerung ausgeschöpft und nutzbar gemacht werden. ${ }^{355} \mathrm{Zu}$ diesem Zwecke solle eine Differenzierung des Ausbildungssystems ${ }^{356}$ durch unterschiedliche Bildungszweige und Lehrgänge gestaltet werden, nämlich:

(1) Vermittlung von wissenschaftlichen und technischen Fachkenntnissen durch eine Fachhochschule oder Universität, die sich nicht unbedingt auf die Ausbildung von handwerklichen Fähigkeiten bezog, sondern für Ingenieure, Manager u. a. gedacht war;

(2) Vermittlung von Fachkenntnissen und Ausbildung handwerklicher Fähigkeiten für Techniker durch eine Fachschule;

(3) gewerbliche Ausbildung zur Vermittlung von Geschicklichkeit und Fertigkeit, etwa einfache Arbeitskräfte bzw. Handwerker; Fachausbildung für Anlernkräfte durch kurzzeitige Heranbildungskurse im Betrieb oder Fortbildung; Fachausbildung für Ungelernte Anweisungen während der Arbeit. ${ }^{357}$

\subsubsection{Unterstützung durch die US-Entwicklungshilfe}

Der Aufbau der beruflichen Schulen sowie der naturwissenschaftlichen und technischen Ausbildung wurde bald behindert, denn es fehlte sowohl an Erfahrung als auch an finanziellen Mitteln, zugleich mangelte es an Lehrkräften und -materien.

Doch nicht nur die KMT-Regierung hatte Interesse an Facharbeitskräften im naturwissenschaftlichen und technischen Bereich, sondern auch die USA, vor allem

\footnotetext{
355 Vgl. 5.5.1.2. in dieser Arbeit.

356 Der Begriff der Differenzierung (in Englisch: Tracking) findet im internationalen Sprachgebrauch eine weite, häufig unscharfe Anwendung, von der Bezeichnung für die Berücksichtigung individueller Unterschiede und für Gruppierungsformen im Unterricht in einer „Klasse“ bis zur Kennzeichnung der Organisation unterschiedlicher allgemein- und berufsbildender Erziehungswege im Ganzen eines Bildungssystems. Vgl. Saul B. Robinsohn und Helga Thomas1971.

357 Vgl. Ku Po-yen 1958.
} 
nach dem „Sputnik-Schock“358 im Jahr 1957. Die Gestaltung des taiwanesischen Ausbildungssystems erhielt ab 1952/53 durch US-amerikanische Berater und durch das von den USA geförderte Projekt für die Verbesserung der naturwissenschaftlichen Bildung neue Impulse.

1947 hatte US-Außenminister George C. Marshall ein Programm für den Wiederaufbau der Wirtschaft von Westeuropa gefordert. Ziel des Marshall-Plans offiziell „European Recovery Program” (ERP) - war erstens die Hilfe für die notleidende und hungernde Bevölkerung des kriegszerstörten Europas, zweitens eine Eindämmung des kommunistischen Einflusses der Sowjetunion und drittens die Schaffung eines Absatzmarktes für die amerikanische Überproduktion. Via Entwicklungshilfe wurden US-amerikanische Modelle, u. a. die landwirtschaftliche und industrielle Produktion, Managementsysteme, Kenntnisse in Naturwissenschaft und Technik und Lebensweise, auf europäische Länder übertragen. Zugleich wurde damit der politische Einfluss der USA gestärkt. ${ }^{359}$ Die zunächst auf Europa beschränkte Außenpolitik wurde später „,zur globalen Strategie“ der USA. ${ }^{360}$

China wurde zwar ab 1948 in den Marshallplan einbezogen, erhielt aber wegen des

\footnotetext{
${ }^{358}$ Der Start des sowjetischen Satelliten Sputnik war ein Zeichen dafür, dass die Sowjetunion die USA technologisch überholt hatte, im Besitz von starken Interkontinentalraketen war und die USA mit Atombomben bedrohen konnte. Daraufhin wurden in den USA verschiedene Programme zur Förderung der bislang als Bildungsreserve vernachlässigten Bevölkerungsgruppen sowie zur Förderung der wissenschaftlich-technischen Entwicklung und der naturwissenschaftlichen Bildung in der Schule aufgelegt. Gleichzeitig verstärkte die Sowjetunion mit massiven Unterstützungsprogrammen die Aufrüstung auf dem chinesischen Festland und anderen kommunistischen Ländern. Auch deswegen hatten Wissenschaftler in den USA ihre Regierung dazu aufgefordert, die Entwicklung der Wissenschaft in nicht westlichen Ländern zu unterstützen, um die soziale und wirtschaftliche Lage dieser Länder zu verbessern und sie zugleich friedlich in die westliche Gesellschaft bzw. den von den USA geleiteten Teil der Welt zu integrieren. Dieser Vorschlag gewann die Unterstützung des damaligem Präsidenten Eisenhower, und kurze Zeit später wurde das „Science Advisory Committee“ gegründet. Vgl. W.A. Jr. Noyes 1958, S. 19-29; Igor J. Polianski und Matthias Schwarz (Hrsg.) 2009.

359 Vgl. Charles A. Thomson und Walter H.C. Laves 1963, S. 91f. sowie Wen Hsing-ying 1989, S. 3034.

${ }^{360}$ Vgl. Gilbert Rist 1997, S. 70-72.
} 
Misstrauens gegenüber dem KMT-Regime keine konkrete Förderung. ${ }^{361}$ Erst zu Beginn des Koreakrieges (1950-1953) begann die Zusammenarbeit zwischen der US-Regierung und dem KMT-Regime. ${ }^{362}$ Nach dem Koreakrieg wurde Taiwan als eine der asiatischen Fronten gegen die kommunistische Expansion betrachtet. Die US-Regierung unterstützte deshalb das Regime Chiang Kai-sheks und stellte zwischen 1950 und 1965 insgesamt um die 1,48 Mrd. USD zur Verstärkung der Verteidigungskräfte sowie zur Entwicklung Taiwans zur Verfügung. Damit sollten Industrie und Agrarwirtschaft unterstützt, die Infrastruktur ausgebaut und die Gesellschaft belebt werden. ${ }^{363}$ Zugleich hatte die US-Entwicklungshilfe die Aufgabe, technische Hilfe und Kooperationen zu ermöglichen, um die Ausbildung der Facharbeitskräfte und den Aufbau des Bildungswesens zu unterstützen.

US-Entwicklungshilfe von 1951 bis 1965, dadurch übte die US-Regierung jedoch auch starken Druck auf die KMT-Regierung aus: Einerseits unterstützten sie Taiwan durch Hilfslieferungen, andererseits forderten sie die KMT-Regierung auf, eine Reihe von Wirtschaftsplänen zu erstellen und durchzusetzen, um einen effektiven Einsatz der Hilfsgüter zu gewährleisten. Im Folgenden wird der Prozess der Wirtschaftsentwicklung in Taiwan in vier Phasen geschildert: 1. Wiederaufbauphase (1945-1960), 2. Expansionsphase des Exports (1960-1972), 3. Umstellungs- und Aufschwungsphase (1973-1986), 4. Liberalisierungsphase (1987 bis zur Gegenwart).

Zwischen 1951 bis 1965 entwickelten US-amerikanische Berater der Education Division in der „U.S. Mutual Security Agency to China“ (MSA) die Programme zum Aufbau des taiwanesischen Bildungswesens; diese bezogen sich auf die primäre und

\footnotetext{
361 Vgl. Tseng Chien-min 2009, S. 62-67.

362 Vgl. Chao Chi-chang 1985, S. 11 und Chang Shu-ya 1990, S. 481.

363 Vgl. Dennis Van Vranken Hickey 1994, S. 21.
} 
sekundäre Bildung sowie Fachausbildung, technische, agrarwirtschaftliche und berufliche Ausbildungen jeglicher Art, naturwissenschaftliche und technische Bildung und Forschung, Erziehung für die Überseechinesen, Medienerziehung, kommunale Erziehung, Hauswirtschafterziehung und schulische Verwaltung. ${ }^{364}$ Von 1951 bis 1971 wurden 3003 taiwanesische Fachleute aus den Bereichen Landwirtschaft, Industrie, Bildung etc. zur Aus- und Weiterbildung in die USA entsandt. ${ }^{365}$ Das Bildungsprogramm wurde durch 148,2 Millionen USD unterstützt, was etwa 2,5\% der gesamten US-Entwicklungshilfe ausmachte. Hinzu kamen Spenden aus privaten Stiftungen in den USA. ${ }^{366}$ Von diesem Geld standen 35\% für die berufliche und 19\% für die naturwissenschaftliche Bildung, 11\% für die Erziehung der Überseechinesen und 35\% für andere Bereiche zur Verfügung. ${ }^{367}$

\subsubsection{Das Gutachten US-amerikanischer Berater}

Unter der KMT-Regierung wurde die noch unter japanischer Besatzung gestaltete fünfjährige Vocational High School aufgeteilt in eine dreijährige Junior Vocational High School und eine dreijährige Senior Vocational High School, wobei mehr als die Hälfte der Schüler in der Junior Vocational High School ihre Vorbereitung auf den Besuch der Senior High Schools oder Senior Vocational High Schools und nicht auf einen künftigen Beruf ausrichtete. Nur knapp 20\% der Absolventen gingen direkt in einen Beruf, und sie mussten jedoch wegen ihrer mangelnden Berufstätigkeit eine zusätzliche Ausbildung in der Arbeit nehmen. ${ }^{368}$ Die mangelnde Koordination zwischen schulischer Berufsausbildung und realem Bedarf sowie die dadurch entstehende Arbeitslosigkeit wurden von den US-Beratern während der

\footnotetext{
364 Vgl. Council for International Economic Cooperation und Development (Hrsg.) 1964, S. 1.

365 Vgl. Chao Chi-chang 1985, S. 29f.

366 Vgl. Fu Li-yuh 2006 (a), S. 336.

367 Vgl. An Hou-wie 1998, S. 68.

368 Vgl. Bildungsbehörde in der Provinz Taiwan (Hrsg.) 1951, S. 82.
} 
„Sino-amerikanischen Konferenz für gewerbliche Erziehung“ 1953 thematisiert und der Plan gefasst, zunächst einmal eine enge Verbindung zwischen Ausbildung und Bedarf aufzubauen. ${ }^{369}$

Zur effektiven Verwendung der US-Entwicklungshilfe wurde daraufhin von Beratern der „J. G. White Engineering Corporation of New York“370 ein „Entwurf für die Industrieplanung der Abrechnungsjahre 1952-55“ erstellt, in dem die Ausbildung von Facharbeitern geregelt wurde, nachdem der Mangel an qualifizierten Facharbeitskräften problematisiert worden war. Die Gründe dafür sah man in der zu theoretischen Ausbildung in den gewerblichen Schulen, sodass es den Schülern an Fertigkeiten und Geschicklichkeit mangele. Zudem könnten die Absolventen weder als Techniker noch als Facharbeitskräfte eingesetzt werden. Weiterhin gebe es eine große Lücke auf dem Arbeitsmarkt zwischen qualifizierten Technikern und einfachen Arbeitern, die nur durch eine planmäßige Ausbildung geschlossen werden könne. ${ }^{371}$

Die Berater entwickelten einen konkreten Plan hinsichtlich der Ausbildungsziele und -zeiträume, der Lehrpläne und der anzustrebenden Zahl an Lehrlingen. ${ }^{372}$ Dabei versuchten sie, das neue Ausbildungsmodell, das zu jener Zeit in den USA erfolgreich praktiziert wurde, auf das taiwanesische Bildungswesen zu übertragen. Konkret gestaltete sich das folgendermaßen: In der Berufsausbildung sollten nur noch die Fachkenntnisse eines ganz bestimmten Ausbildungsberufs vermittelt werden. Danach konnten die Schüler sofort an einer entsprechenden Arbeitsstelle eingesetzt werden.

\footnotetext{
${ }^{369}$ Vgl. Bildungsministerium (Hrsg.) 1957, S. 346; Fang Chun-yu 2000, S. 61;Chung Han-shu 2004, S. 59 und Udo Dörnhaus 1988, S. 57f.

${ }^{370}$ Eine Gesellschaft für Infrastruktur, die durch Beratung und Abrechnung der Wirtschaftsprojekte der US-Entwicklungshilfe in Taiwan half. 1962 wurde sie zur „Engineering Consulting Group” unter dem "Council for U.S. Aid" umstrukturiert. Vgl. Chao Chi-chang 1985, S. $276 f$.

371 Vgl. An Hou-wei 1998, S. 15-17.

372 Vgl. ebd., S. 14-16.
} 
Dieses Ausbildungskonzept wurde als „Unit Trade Training“ bezeichnet, da sich die Ausbildung an eng umgrenzten Gewerbesparten orientierte. So sollte auch in Taiwan eine enge Verknüpfung von Schulen und Betrieben ermöglicht werden.

\subsubsection{Aufbau der beruflichen Ausbildung}

Ab 1953 traten sog. „Vierjahrespläne zum wirtschaftlichen Aufbau“ in Taiwan in Kraft. Die vorrangige Entwicklungsstrategie des ersten und zweiten Plans (1953-1956 und 1957-1960) war, „die Industrie mit Hilfe der Landwirtschaft zu entwickeln und die Landwirtschaft durch Unterstützung der Industrie auszuweiten“. Das heißt, die Bauern sollten einen eigenen Acker besitzen, und die Produktionstechniken sollten verbessert werden, um dadurch die Landwirtschaftsproduktion zu erhöhen und mit Gewinnen durch Exporte die für die industrielle Entwicklung benötigten importierten Maschinen, Anlagen und Rohstoffe zu finanzieren. Die Schwerpunkte der industriellen Entwicklung in der Textilindustrie, Konsumgüterindustrie und Lebensmittelindustrie lagen primär darauf, den Bedarf der Bevölkerung zu decken. ${ }^{373}$ Um den Bedarf an Arbeitskräften gemäß den Wirtschaftsplänen zu decken, forderten die US-Berater einen Umbau der beruflichen Ausbildung, vor allem im gewerblichen und landwirtschaftlichen Bereich. Der Entwurf gewann die Anerkennung der „U.S. Mutual Security Mission to China“ und der „U.S. Agency for International Development", sodass ein Entwicklungshilfe-Fonds zum Zweck des Aufbaus der Berufsausbildung in Taiwan angelegt wurde. Zunächst wurden die Ausbildungsstätten für die Lehrkräfte aufgebaut, danach die Errichtung von gewerblichen Schulen und Schulen für die landwirtschaftlichen Bereiche in den Vordergrund gestellt.

${ }^{373}$ Vgl. Wirtschaftsministerium (Hrsg.) 1971 (a) und (b). 


\subsubsection{Aufbau der Lehrerausbildung und Umorganisation der Fachhochschulen}

Unter der japanischen Besatzung waren zwar Einrichtungen für die Ausbildung von Technikern und einfachen Arbeitskräften wie Fachschulen, berufliche Schulen und Ausbildungseinrichtungen für kurzzeitige Förderungskurse aufgebaut worden, ${ }^{374}$ jedoch kamen die meisten Lehrkräfte aus Japan, und es wurde keine Institution für Lehrkräfte der beruflichen Ausbildung eingerichtet. ${ }^{375}$ Obwohl die KMT-Regierung versuchte, Lehrkräfte vom chinesischen Festland zu rekrutieren, entstand wegen der raschen Ausweitung der Bildungsstätten ein kritischer Mangel an Lehrkräften. Mit der US-Entwicklungshilfe wurden zunächst Einrichtungen für die Lehrerausbildung finanziert und zugleich Fachberater nach Taiwan entsandt. ${ }^{376}$ Im Jahre 1953 wurde mit Unterstützung der Pennsylvania State University eine Abteilung für gewerbliche Pädagogik an der Pädagogischen Fachhochschule Taiwan errichtet, in der Lehrkräfte für das „Unit Trade Training“ ausgebildet wurden. ${ }^{377} \mathrm{Zu}$ dieser Zeit kamen auch Bildungsberater der Purdue University nach Taiwan, um bei der Umorganisation der Technischen Fachhochschule der Provinz Taiwan zu helfen. An der Pädagogischen Fachhochschule wurde die Fakultät für Haushaltswesen ${ }^{378}$ errichtet und die Lehrkräfte zur Pennsylvania State University zur Fortbildung entsandt. ${ }^{379}$

\subsubsection{Umbau der gewerblichen Schulen}

Zur Reorganisation der Berufsschulen benötigte man empirische Daten über die Arbeitsteilung in der Berufswelt und die Arbeitskräfte in den Fabriken. ${ }^{380}$ Ab 1955 wurde das Modell des „Unit Trade Training“ zunächst an acht gewerblichen Junior

\footnotetext{
${ }^{374}$ Vgl. 4.2.3. in dieser Arbeit.

375 Vgl. Li Yuen-huei 1984, S. 338-344 und Bildungsministerium (Hrsg.) 1957, S. 364.

376 Vgl. Udo Dörnhaus 1988, S. 57 und 60f.; Chao Chi-chang 1985, S. 188.

377 Vgl. National Institute of Educational Resources and Research (Hrsg.) 1957, S. 1-25.

378 Vgl. ebd., S. 40f.

379 Vgl. Chao Chi-chang 1985, S. 214-217.

380 Vgl Yang Hsien-guo 1969, S. 1 und Chung Han-shu 2004, S. 58f.
} 
High Schools als Modellschulen mit 33 unterschiedlichen Berufsausbildungen eingeführt, ${ }^{381}$ welche nun stärker auf den realen Bedarf in der Berufswelt ausgerichtet waren. ${ }^{382}$ Zunächst wurden Schulgebäude und Ausrüstungen sowie Einrichtungen finanziert. Außerdem stellte jeder „Unit Trade“ eine Verbindung mit entsprechenden Betrieben her, um den Schülern Praktikumsmöglichkeiten zu bieten. In jener Zeit gab es noch kaum private Unternehmen auf Taiwan, daher beschränkten sich die Kooperationsprojekte auf staatliche Schiffbaubetriebe, Elektrizitätsgesellschaften, Eisenbahngesellschaften und Rüstungsanlagen. ${ }^{383}$

\subsubsection{Aufbau landwirtschaftlicher Berufsschulen}

Die Ziele der landwirtschaftlichen Ausbildung unterschieden sich von anderen Berufsausbildungen, nicht nur moderne Landwirte wurden hier ausgebildet, sondern auch Führungskräfte. Zugleich stellte sie eine Vorbereitung auf das Studium der Agrarwissenschaft dar, die Absolventen hatten die Aufgabe, ihre Kenntnisse weiterzuvermitteln und die Bauern $\mathrm{zu}$ organisieren, damit die gesamte landwirtschaftliche Produktion sowie die Lebensbedingungen verbessert werden konnten. ${ }^{384}$ Die Modernisierung des ländlichen Lebens mitsamt Verbesserungen der hygienischen Bedingungen und der Geburtshilfe war einer der Schwerpunkte des Projekts „Ländliche Rekonstruktion“, das Aufbauprojekt wurde unter der Leitung der „Sino-American Joint Commission on Rural Reconstruction“ durchgeführt. ${ }^{385}$

\footnotetext{
381 Vgl. Udo Dörnhaus 1988, S. 59f.

382 Vgl. Yang Hsien-guo 1969, S. 1 und Chung Han-shu 2004, S. 58f.

383 Vgl. An Hou-wei 1998, S. 164-172.

384 Vgl. National Institute of Educational Resources and Research (Hrsg.) 1957, S. 37 und ders. 1959, S. 36f. Vgl. auch Chung Han-shu 2004, S. 87-94.

${ }^{385}$ Die JCRR, eine Unterabteilung der US-Entwicklungshilfe, wurde 1948 gegründet und stellte über $10 \%$ der US-Entwicklungshilfe der ,ländlichen Rekonstruktion“ zur Verfügung. Aufgaben waren die Förderung der Landwirtschaft, Durchführung der Landreform und Vermittlung sowie Verbreitung von Technik und neuer Konzepte, um die Produktionskapazität zu erhöhen und die Ausbildung der Facharbeitskräfte bzw. Führungskräfte für die Durchführung der Reformprojekte sicherzustellen. Vgl. Chao Chi-chang 1985, S. 115-118 und „Sino-American Joint Commission on Rural Recon-
} 
Außerdem wurde seit 1952 das System des „4-H Club“ (Head, Heart, Hands and Health) auf die taiwanesische Landwirtschaftserziehung übertragen; es bildete eine Verbindung zwischen der Landwirtschaftsschule und der lokalen Gemeinde und bot zugleich Praktikumsmöglichkeiten für Schüler aus landwirtschaftlichen und Haushaltsschulen. $^{386}$

Ab 1954 wurden 25 landwirtschaftliche Senior High Schools in dieses Programm einbezogen. 1955 begann die Universität Michigan eine wissenschaftliche Zusammenarbeit mit der Fakultät für Landwirtschaft an der „National Taiwan University" und der neu gegründeten Abteilung für Landwirtschaftsausbildung an der Fachhochschule für Agrarwissenschaft in Taichung, um Lehrkräfte fortzubilden. ${ }^{387}$ In die „ländliche Rekonstruktion“ wurde nun auch die Ausbildung in Hauswirtschaft und Krankenpflege integriert. Mit Unterstützung der Pennsylvania State University wurde 1953 die Fakultät für Hauswirtschaft an der Pädagogischen Fachhochschule errichtet, um Lehrkräfte und Erzieherinnen zur Vermittlung der neuen Kenntnisse in der Hauswirtschaft auf dem Land ausbilden. Der Lehrgang Hauswirtschaft wurde nicht nur in drei Schulen für Haushaltswesen, sondern auch in vier High Schools für Mädchen und allen landwirtschaftlichen Schulen eingesetzt. Ab 1956 wurde es Pflichtfach mit zwei Stunden pro Woche in allen High Schools für Mädchen. ${ }^{388} 1952$ wurde die erste unabhängige Schule für Krankenpflege in Taiwan errichtet. Die Ausbildung ließ sich oft mit lokalen Hygienestationen kombinieren.

struction“ (JCRR) (Hrsg.) 1951, S. 2.

386 Vgl. ebd., S. 197-199.

387 Vgl. National Institute of Educational Resources and Research (Hrsg.) 1957, S. 41f. sowie Chen Tien-hsi, Yu Huan-mo und Chang Pi-chi 1983, S. 81f.

388 Vgl. Bildungsministerium (Hrsg.) 1974, S. 591. 
Der zweite „Vierjahresplan zum wirtschaftlichen Aufbau“ (1957-1960) ${ }^{389}$ bezog sich nicht nur auf die Landwirtschaft, sondern auch auf das Meer. Ab 1957 wurde der Lehrgang für Fischerei und Meereswesen in der High School einer der Schwerpunkte der US-Entwicklungshilfe, ${ }^{390}$ und drei Berufsschulen, in Kaohsiung, Penhu und Keelung sowie eine private Fachschule (1966) in Taipei wurden eingerichtet. ${ }^{391}$

\subsubsection{Aufbau naturwissenschaftlicher und technischer Forschung und Aus bildung}

$\mathrm{Zu}$ Beginn zögerte die KMT-Regierung noch, langfristig in die Entwicklung der Wissenschaften zu investieren, denn es war nicht sicher, wie lange sie in Taiwan bleiben würde. Im Vordergrund standen die „Bildung des Volksgeistes“ und die militärische Erziehung. Die naturwissenschaftliche Ausbildung wurde wegen des Mangels an Lehrkräften stark vernachlässigt, die US-amerikanische Forscherin J. Megan Greene sprach von einer „Wissenschaftswüste““: „Scientific Desert““ ${ }^{392}$

\subsubsection{Historischer Rückblick}

1914 gründeten der Chemiestudent Jen Hung-chuan ${ }^{393}$ und andere Austauschstudenten, die aufgrund des Boxerprotokolls an der Cornell Universität in den USA studierten, die „China Science Society“, eine der einflussreichsten Gesellschaften für Wissenschaft vor 1949 in China, die bis 19493776 Mitglieder aus unterschiedlichen Fachgebieten zählte. Als Jen und andere Mitglieder der „China Science Society“ 1918 nach China zurückkehrten, begannen sie mit der Verwirklichung ihres Projekts zur

\footnotetext{
389 Vgl. Wirtschaftsministerium (Hrsg.) 1971 (a) und (b).

390 Vgl. An Hou-wei 1998, S. 40f.

391 Vgl. Bildungsministerium (Hrsg.) 1974, S. 657.

392 Vgl. J. Megan Greene 2000, S. 71-76.

393 Jen Hung-chuan (1886-1961) studierte von 1912 bis 1918 in den USA Chemie, anschließend war er Professor an der Universität Peking, Präsident der Universität Ssu-chuan, Präsident des „China Foundation for the Promotion of Education and Culture“ sowie Dekan des Chemie-Instituts an der „Academia Sinica“. Vgl. Yang Tsui-hua 1995, S. 297-324.
} 
„Verbreitung der naturwissenschaftlichen Kenntnisse und zur Verankerung der Forschung und Ausbildung der Forschungskräfte in China“. Ab 1924 unterstützte die „China Foundation for the Promotion of Education and Culture“ 394 auch die naturwissenschaftliche Bildung in der Schule sowie Forschungsprojekte und die Ausbildung von Fachkräften, vor allem als Jen die Stiftung von 1935 bis 1949 leitete. ${ }^{395}$ 1928, als die Nankinger KMT-Regierung an der Macht war, forderten Wissenschaftler und Gelehrte, die im Ausland studiert hatten, eine staatliche Unterstützung für Forschung und Ausbildung, in der Folge wurde die „Academia Sinica“" mit zwölf Forschungsrichtungen gegründet. ${ }^{396}$

Ein kleiner Teil der Mitglieder der „Academia Sinica“, darunter Hu Shih, Mei I-chi ${ }^{397}$, Wu Ta-you ${ }^{398}$ und Chien Ssu-liang ${ }^{399}$, waren mit dem KMT-Regime nach Taiwan emigriert und versuchten hier ihre wissenschaftlichen Kenntnisse einzubringen. 1953 gründeten Wissenschaftler der „Academia Sinica“ die „Chinese Association for the Advancement of Science“ (CAAS) auf Taiwan, nachdem Li Kuo-ting ${ }^{400}$, Chou

\footnotetext{
${ }^{394}$ Im Jahr 1908 wurde ein „Sino-America Komitee“ gegründet, das Stipendien an ausgewählte chinesische Studenten verteilte, und es wurde das Tsing Hua College in Peking errichtet, das als Vorschule für in die USA entsandte Studenten zur Verfügung stand. 1924 wurde die „China Foundation for the Promotion of Education and Culture“ zur Verwaltung und Verteilung der „Entschädigungsgelder gemäß dem Boxerprotokoll“ von Seiten der USA gegründet. Vgl. Shu Hsin-cheng 1989, S. 72-86 und 248-260; Wang Bing-chao 1994, S. 241-245 und Yang Tsui-hua 1989, S. 259-280.

${ }_{395}$ Vgl. Yang Tsui-hua 1991, S. 115-206 und ders. 1995, S. 316-319.

396 Vgl. Hsu Ming-hua 1993, S. 244 f.

397 Mei I-chi (1889-1962) war später Physikprofessor und Präsident an der staatlichen Tsing Hua Universität sowie Bildungsminister und Mitglied der „Academia Sinica“.

${ }^{398}$ Wu Ta-you (1907-2000) war Physikprofessor und Präsident der „Academia Sinica“.

399 Chien Ssu-liang (1908-1983) war später Chemieprofessor an der Universität Peking und Präsident der staatlichen Universität Taiwan (1951-1970) sowie Präsident der „Academia Sinica“ (1970-1983).

${ }^{400}$ Li Kuo-ting (1910-2001) studierte von 1934 bis 37 an der Universität Cambridge Physik. Seit 1953 arbeitete er im „Council for U.S. Aid” (CUSA), wurde Chef-Sekretär des CUSA und ab 1963 Vizevorsitzender des „Council for International Economic Cooperation und Development” (CIECD). 1965 trat Li das Amt als Wirtschaftsminister an, war Berater des Staatssicherheitsrats und von 1969 bis 1976 Finanzminister, anschließend war er Berater der staatlichen Wissenschaftskommission (19762001), der Wirtschaftskommission (1977-1988) und Staatsberater (1988-2001) usw. Er hatte großen Anteil an der Industrialisierung und Modernisierung Taiwans. 1994 wurde er als „Vater der Technologie auf Taiwan“" geehrt.
} 
Hung-ching ${ }^{401}$ und Tai Yun-kui ${ }^{402}$, Vertreter der Fachgebiete Mathematik, Physik, Chemie, Astronomie u. a., 1951 die „Chinese Association for the Advancement of Nature Science“ (CAANS) gegründet hatten. ${ }^{403}$ Die emigrierten Wissenschaftler strebten die „Autonomie der Forschung“ an und übernahmen Verantwortung für die Entwicklung des Landes. Sie versuchten, naturwissenschaftliche Fachdisziplinen auf Taiwan zu etablieren, um ihr Ideal der „Rettung des Landes mit Hilfe der Wissenschaft" realisieren zu können, ${ }^{404}$ und kümmerten sich insbesondere um den Nachwuchs. 1964 formulierte der Präsident der „Academia Sinica“, Wang Shih-chieh (1891-1981), dass „Lehrkräfte, Dozenten und Wissenschaftler an den Universitäten und in den Forschungsinstituten eigenständig auf Taiwan ausgebildet werden sollten und dass die Universitäten und Forschungsinstitute Führungskräfte gemäß dem Bedarf von Wirtschaft und Industrie ausbilden sollten“. ${ }^{405}$ Als Voraussetzung für die Verwirklichung dieser Ziele sollten zunächst günstige Arbeitsbedingungen wie Forschungseinrichtungen geschaffen werden. Die Wissenschaftler mit Auslandsbeziehungen und internationalen Kenntnissen suchten hierfür finanzielle Unterstützung der US-Regierung zu gewinnen und Kooperationen mit wissenschaftlichen Gesellschaften in den USA herbeizuführen.

\subsubsection{Die taiwanesisch-amerikanische Kooperation}

Infolge dieser Bemühungen wurde 1959 eine Ad-hoc-Kommission für Forschung und Ausbildung von Forschungskräften - „The National Council on Long-range Science

\footnotetext{
401 Chou Hung-ching (1902-1957) studierte 1934 bis 1937 an der Universität London Mathematik, war später Mathematik-Professor und hoher Beamter im Bildungsministerium (1945 bis 1948), und Dekan des Mathematik Instituts der „Academia Sinica“.

402 Tai Yun-kui (1897-1882) studierte 1922 bis 1927 an der Kaiserlichen Universität Kyoto in Japan Physik und wurde Physikprofessor. Er trug wesentlich zum Aufbau der physikalischen Forschung auf Taiwan bei und wurde als ,Vater der Physik auf Taiwan“ verehrt.

${ }^{403}$ Vgl. Fu Li-yuh 2006 (a), S. 339 und ders. 2006 (b), S. 449.

404 Vgl. ders. 2006 (a), S. 339-341.

405 Vgl. Yang Tsui-hua 1999, S. 52 und ders. 2002, S. 343.
} 
Development“ (1959-1967) - unter Aufsicht des Exekutivrates bzw. der „Academia Sinica“" und des Bildungsministeriums gegründet, um eine Förderung sowie die Zusammenarbeit zwischen US-amerikanischen und taiwanesischen Wissenschaftlern zu ermöglichen. 1967 wurde sie zu der langfristigen Kommission „The National Council on Scientific Development" (NCSD) umorganisiert. Diese war mit der Durchführung der Pläne des „Direct Council for Development of Science“ betraut, wodurch jegliche Forschungsaktivität und -entwicklung direkt unter Aufsicht von Präsident Chiang Kai-shek stand.

1953 hatte US-Präsident Eisenhower in der UNO mit der Devise „Atoms for Peace“ die internationale Zusammenarbeit in der Atomforschung gefordert. 1954 finanzierte die „USA Atomic Energy Commission“ die Hälfte der Ausgaben für die Errichtung eines Atomreaktors in Taiwan. Um dieses Projekt durchzuführen, wurde 1954 eine „Kommission für naturwissenschaftliche Bildung“ im Bildungsministerium und ein von Wu Ta-you geleiteter „Atomic Energy Council“ innerhalb des Exekutivrates sowie ein Institut für Kernkraftforschung an der staatlichen Tsing Hua Universität errichtet. Unter dem Eindruck des „Sputnik-Schocks“ im Jahr 1957, der in den USA hektische Aktivitäten zur Schließung der vermeintlichen technologischen Lücken auslöste und auf die Förderung des Nachwuchses gerichtet war, wurden auch auf Taiwan Überlegungen angestellt, wie durch verstärkte Investitionen in die Ausbildung hoch qualifizierter Arbeitskräfte ein entsprechendes Wirtschaftswachstum erzielt werden könne. Auch in den Förderprojekten der USA wurde auf die kargen Bedingungen für wissenschaftliche Forschung aufmerksam gemacht. ${ }^{406}$ Dieses Problem wurde vor allem von Hu Shih, 1957 zum Präsidenten der „Academia Sinica“" gewählt, thematisiert. Er war aus den USA nach Taiwan übergesiedelt, um 406 Vgl. Bildungsministerium (Hrsg.) 1974, S. 917. 
dort sein wissenschaftliches Programm fortzusetzen. Seit 1958 predigte er die Notwendigkeit einer Wissenschaftsentwicklung des Landes und forderte günstige Arbeitsbedingungen und eine Verbesserung der naturwissenschaftlichen Bildung in der Schule zum Zweck der Wiedereroberung des verlorenen chinesischen Festlandes und der künftigen Entwicklung des Landes. Daher sollte die KMT-Regierung trotz des sehr begrenzten Staatshaushalts die Entwicklung von Naturwissenschaft und Technik unterstützen. ${ }^{407} \mathrm{Hu}$ und seine Kollegen in der „Academia Sinica“ schlugen vor, ein „Long-range Science Development Program“ zu erstellen, ${ }^{408}$ woraufhin „The National Council on Long-range Science Development" gegründet wurde. Das zugehörige Programm sollte unter Aufsicht der „Academia Sinica” und des Bildungsministeriums durchgeführt werden. Die Ziele waren, finanzielle Mittel aufzubringen, Forschungsstellen an den Universitäten und Instituten zu gestalten, Gastprofessuren zu organisieren, Siedlungen für Letztere zu errichten und Beihilfen zu Publikationen der wissenschaftlichen Forschung zur Verfügung zu stellen. ${ }^{409}$

Zu Beginn hatte die KMT-Regierung die Kommission nur sehr begrenzt gefördert, die Mitglieder mussten für die finanziellen Mittel also selbst sorgen. Dazu wurde ein Antrag auf „Unterstützungs- und Austauschprogramme für die Wissenschafts- und Technologieentwicklung in Taiwan“ bei „The Council for United States Aid“ (CUSA) eingereicht und anerkannt. ${ }^{410}$ Zugleich bemühte sich Hu um finanzielle Unterstützung der „China Foundation for the Promotion of Education and Culture“411 und der „Asia

\footnotetext{
407 Vgl. Fu Li-yuh 2006 (a), S. 343.

408 Vgl. Yang Tsui-hua 2002, S. 333-337.

409 Vgl. Bildungsministerium (Hrsg.) 1974, S. 917-919.

410 Vgl. Yang Tsui-hua 2002, S. 338 und J. Megan Greene 2000, S. 78-85.

411 Nach Gründung der VR China war Hu Shih in die USA emigriert und hatte diese Stiftung mit den anderen emigrierten chinesischen Wissenschaftlern verwaltet, damit die „Entschädigungsgelder gemäß dem Boxerprotokoll“" vonseiten der USA zur Unterstützung der Forschung und naturwissenschaftlichen Bildung für Chinesen weiterhin fließen konnten. Vgl. Yang Tsui-hua 1989, S. 259-280 und ders. 2002, S. 327-352.
} 
Foundation“, 412 und von 1959 bis 1964 erhielt die Kommission insgesamt ca. 1,4 Millionen USD. ${ }^{413}$ Durch diese Fremdmittel konnte schließlich das „Long-range Science Development Program“ durchgeführt werden.

Nachdem die „National Academy of Science“ (NAS) Taiwan als Partnerland für die wissenschaftliche Zusammenarbeit im pazifischen Raumes ausgewählt hatte, weil es eine relativ gute Bildungsbasis habe und sich gerade um die Verbesserung der naturwissenschaftlichen Bildung und der Forschung bemühe und dies somit zu einer erfolgreichen Zusammenarbeit führen zu könne, ${ }^{414}$ gründete sie in der „SinoAmerican Conference of Intellectual Cooperation 1966“ gemeinsam mit der „Academia Sinica“ den „China Council of Sino-American Cooperation on Science“. Zwei Jahre später schloss sich die „Academia Sinica“ mit dem „American Council of Learned Societies“, dem „Social Science Research Council“ sowie dem „China Council of Sino-American Cooperation on Humanities and Social Science“ zusammen, was zum Austausch der Wissenschaftler und zur Beförderung der wissenschaftlich-institutionellen Zusammenarbeit zwischen beiden Ländern beitragen sollte. Mit Unterstützung des "The Sino-American Economic and Social Development Funds" wurden Institute für Mathematik, Physik, Chemie, Biologie, Technologie und später auch Meereskunde (1968) errichtet; zudem entstanden Institute für Wirtschaftswissenschaft (1967) und US-amerikanische Kultur (1972). ${ }^{415}$ 1969 wurde ein „Abkommen für naturwissenschaftliche und technische Zusammenarbeit zwischen Taiwan und den USA“ abgeschlossen, um den Austausch

\footnotetext{
${ }^{412}$ Die „Asia Foundation“ wurde 1956 von der,„Central Intelligence Agency“ in den USA gegründet. Sie bot ein Austauschprogramm für Wissenschaftler und die Förderung von Forschungsprojekten innerhalb der „Academia Sinica“, außerdem auch Ausbildungsprogramme für Naturwissenschaft, Soziologie und Agrarwissenschaft. Vgl. Yang Tsui-hua 2002, S. 338.

413 Vgl. J. Megan Greene 2000, S. 82.

414 Vgl. Yang Tsui-hua 1999, S. 45.

415 Vgl. Bildungsministerium (Hrsg.) 1974, S. 754-757.
} 
innerhalb aller Abteilungen und anerkannten Institute auf Taiwan und in den USA zu ermöglichen. 1978 wurden die Kommissionen zum „Council of Sino-American Cooperation on Science Learning“ zusammengeschlossen.

Die „Chinese Association for the Advancement of Nature Science“ (CAANS) plante seit 1956 die Erstellung von Richtlinien für naturwissenschaftliche Lehrinhalte und Einrichtungen für naturwissenschaftliche Experimente in den Junior und Senior High Schools. Hierzu wurde eine empirische Untersuchung über die Unterrichtssituation in der naturwissenschaftlichen Bildung in 238 Senior High Schools durchgeführt. Auf dieser Grundlage reichten die Forscher 1958 dem Bildungsministerium ein „Programm für die Förderung der naturwissenschaftlichen Bildung in den Senior High Schools“ ein. ${ }^{416}$ Dieses wurde in die „Projekte für die Verbesserung der naturwissenschaftlichen Bildung in dem ersten und zweiten Jahr des Studiums an der Universität sowie in den Senior High Schools“ des „National Council on Long-range Science Development“ einbezogen. Obwohl das „Council for United States Aid“ (CUSA) die Projekte im Prinzip befürwortete, sollten sie nur für die Entwicklung der Hochtechnologie und Forschung eingesetzt werden. Doch die Mitglieder der CAANS waren der Meinung, dass nur durch die Verbesserung der Bildungsqualität und die systematische Vermittlung naturwissenschaftlicher Kenntnisse die gesamte Entwicklung des Landes verbessert werden könne.

In Entsprechung zum „Programm für die Beförderung der Bedingungen für naturwissenschaftliche Bildung in den Senior High Schools“ wurden die Aufgaben neu bestimmt: „Aufbau einer Grundlage für die wissenschaftliche Entwicklung durch

\footnotetext{
416 Vgl. „Chinese Association for the Advancement of Nature Science“ (Hrsg.) 1956, S. 55-56 und ders. 1960, S.59-64.
} 
die Förderung des Naturwissenschaftsunterrichts“, „Förderung der angewandten Wissenschaft zur Deckung des Lebensbedarfs“, „Herausbildung des wissenschaftlichen Denkens durch Wecken der wissenschaftlichen Interessen“ sowie „Effektivität der Arbeit und Produktion mithilfe von wissenschaftlichen Methoden“. Dieses sollte mit folgenden Maßnahmen durchgeführt werden: „Neubestimmung der Lehrinhalte, Ausbildung der Lehrkräfte, Verbesserung der Didaktik sowie Anschaffung von Versuchsmaterialien und unterrichtsbezogenen Ausrüstungen für naturwissenschaftliche Fächer““ ${ }^{417}$

Ab 1959 begannen Fachleute und Lehrer in Taiwan gemeinsam mit Erziehungsberatern der UNESCO, Lehrbücher für Mathematik, Chemie, Physik und Naturkunde zu schreiben. Dieses Projekt wurde schließlich wegen des zu großen Zeitaufwandes aufgegeben und stattdessen eine Übersetzung US-amerikanischer Lehrbücher vorgenommen. 1962 wurde die „Kommission für Übersetzung und Edition sowie Evaluation der mathematischen und naturwissenschaftlichen Lehrstoffe für die Senior High School“" unter Führung des Bildungsministeriums gegründet. Fünf Professoren und vier Lehrer aus den entsprechenden Fachgebieten waren dafür zuständig. Ihre wesentliche Aufgabe war es, die neuesten Lehrbücher der Fächer Biologie, Chemie, Physik, Mathematik, die in den USA bzw. in Japan verwendet worden, zu prüfen, ins Chinesische $\mathrm{zu}$ übersetzen und auf dieser Basis die Lehrbücher für taiwanesische Schüler umzuschreiben. ${ }^{418}$ Die schlechte Übersetzungsqualität und die für taiwanesische Schüler lebensfernen Inhalte erschwerten und überforderten jedoch die Vermittlung und die Aufnahme der Lerninhalte in die Praxis. ${ }^{419}$

\footnotetext{
417 Vgl. Fu Li-yuh 2006 (a) S.348-350 und 368.

418 Vgl. Bildungsministerium (Hrsg.) 1974 S.948f..

${ }^{419}$ Vgl. Chou Chi-wen 1987 S.4-8.
} 
Gemäß dem empirischen Untersuchungsergebnis des CAANS 1959 besaßen 86\% der Lehrer für naturwissenschaftliche Fächer in den Senior High Schools keine entsprechende Fachausbildung. Deswegen wurde eine Reihe von Weiterbildungsprogrammen initiiert und Fortbildungsmöglichkeiten für taiwanesische Lehrer der Naturwissenschaften in den USA gefördert. ${ }^{420}$ Erst in den 1970er-Jahren waren die Lehrer in der Schule allmählich in der Lage, auf der Basis der US-amerikanischen und japanischen Lehrstoffe lebensnahe Lehrinhalte für Mathematik und Naturwissenschaftsfächer neu zu erstellen. ${ }^{421}$

\subsubsection{Regimekritik infolge der wissenschaftlichen Kooperation}

Mit dem „Projekt für die Verbesserung des naturwissenschaftlichen Unterrichts“ wurden zahlreiche Lehrmaterialien und -mittel sowie für naturwissenschaftliche Experimente benötigte Ausrüstungen aus den USA importiert, was zu spürbaren Verbesserungen führte. ${ }^{422}$ Die Durchführung des „Long-range Science Development Program“ schaffte somit die Basis für die Entwicklung der naturwissenschaftlichen Forschung und Ausbildung in Taiwan. Zudem ermöglichte die wissenschaftliche und technische Zusammenarbeit eine Entwicklung in der Ausbildung von Forschungs- und Lehrkräften.

Doch wurde infolge der Kooperation auch eine kritische Haltung gegenüber der sozialen und politischen Entwicklung des Landes gefördert, welche die Loyalität der emigrierten Wissenschaftler wie Hu Shih, Wang Shih-chian, Lei Chen (1897-1979) und Yin Hai-guang (1919-1969) ${ }^{423}$ gegenüber Chiang Kai-shek und der

\footnotetext{
${ }^{420}$ Vgl. Fu Li-yuh 2006 (a), S. 350-357.

421 Vgl. Bildungsministerium (Hrsg.) 1974, S. 950.

422 Vgl. Fu Li-yuh 2006 (a), S. 360f.

423 Yin Hai-guang war Dozent an der philosophischen Fakultät der National Taiwan Universität Yin
} 
KMT-Regierung ins Wanken brachte. ${ }^{424}$ Infolge der Feindschaft zum kommunistischen Regime in China wurden Forscher, die sich mit empirischen sozialwissenschaftlichen Untersuchungen über Taiwan beschäftigten, als Spione oder Anhänger des chinesischen Kommunismus in Haft genommen und verurteilt. ${ }^{425}$ Daher wurde die Forderung nach einem „Zentrum für Forschung über chinesische Kultur und Geschichte, den Kommunismus auf dem chinesischen Festland und die gegenwärtige Entwicklung Taiwans“ der „Academia Sinica“ von der KMT-Regierung sehr kritisch betrachtet.

Eine große Anzahl taiwanesischer Wissenschaftler, die infolge der Austauschprogramme in den USA ausgebildet wurden, lernten dort die US-amerikanische Kultur kennen, trugen dazu bei, deren Werte und Normen auf Taiwan bekannt zu machen, und sorgten dafür, dass diese Einfluss nahmen auf die gesamtgesellschaftliche Entwicklung. Darüber hinaus begeisterten sich nach dem Bericht von H. Neil Jacoby, Beamter bei der „United States Agency for International Development" (US-AID), taiwanesische Jugendliche für die USA, vor allem aufgrund der massiven Investitionen in die naturwissenschaftliche Bildung, neue

Haiguang wurde vor allem durch Bertrand Russell beeinflusst. Yin kann als Vertreter des modernen Empirismus -- im weitesten Sinne -- angesehen werden, der ein großes Vertrauen in die Leistungen der empirischen Wissenschaft besaß. Er zählte zur sogenannten liberalen Opposition der 1950er und 60er Jahre auf Taiwan, die sich um die Zeitschrift „Free China“ gruppiert hatte. Vgl. Chang Chung-tung 1990.

${ }^{424}$ Die Verhängung des Kriegsrechts wurde indes häufig infrage gestellt, und zwar von neuen politischen Gegnern der KMT, den vom chinesischen Festland emigrierten Liberalen, den Vertretern für die radikale Verwestlichung, sowie den Taiwanesen, die ins Exil gegangen waren und sich die Gründung eines von China unabhängigen Staates wünschten. Letztere betrieben ihre Opposition, indem sie Klein- oder Mittelunternehmen förderten und heimlich Widerstandsaktivitäten gegen die KMT-Regierung unterstützten. Hu Shih, Lei Chen, Wang Shih-chieh u.a. versuchten mit der Zeitschrift „Free China“ den „liberalen und demokratischen“ Geist der „Bewegung des Vierten Mai 1919“ auf Taiwan fortzusetzen. Zu Beginn wurde ein für die KMT-Regierung positives Bild durch die Herausgabe dieser Zeitschrift gezeichnet, nämlich als eines Vertreters gegen den chinesischen Kommunismus in der internationalen Gesellschaft. Anschließend forderten diese emigrierten Liberalen nicht nur die Liberalisierung der Wirtschaft, sondern auch den Aufbau eines demokratisch verfassungsgemäßen Politiksystems. Daher kritisierten sie auch die Maßnahmen der faschistischen Herrschaft Chiangs in unterschiedlichen Bereichen. Vgl. Chang Chung-tung 1990; Chan Yoa-chi 2005, S. 30-41; Yen Shu-fang 1989; Su Rui-qiang 2003 und Hsueh Hua-yuan 1995, S. 127-160.

425 Vgl. Yang Tsui-hua 1999, S. 54-59. 
Lernmaterialien und -ausrüstungen, und entwickelten eine Affinität zur US-amerikanischen Kultur. Die USA avancierte zum Traumland für viele Jugendliche. Immer mehr träumten von einem Austauschstudium und planten, falls diese Hoffnung erfüllt würde, sich anschließend dort niederzulassen. Zahlreiche Wissenschaftler, Techniker, Dozenten, Schüler und Schullehrer wurden durch eine Reihe von Austauschprogrammen, etwa dem „Fulbright Act“, zu Studien- und Forschungszwecken in die USA entsandt; im Gegenzug besuchten US-amerikanische Wissenschaftler Taiwan. ${ }^{426}$

Von 1960 bis 1968 hielten sich durchschnittlich jedes Jahr 1500 Studenten in den USA auf, was 22\% der Absolventen auf Taiwan ausmachte; darunter 30\% Studenten der Naturwissenschaften und technischen Fächer. In der Folge entstand das sog. Problem von „brain drain“ (Abwanderung der Wissenschaftler), denn nur unter $4 \%$ der Studenten kehrten nach dem Studium nach Taiwan zurück, da sie hier keine entsprechende Arbeitsstelle finden konnten. Zudem sagte ihnen die Lebensqualität in den USA eher zu, zumal die Forschungsbedingungen besser waren. ${ }^{427}$ Die Abwanderung führte zum Verlust von wissenschaftlichen Mitarbeitern und hoch qualifizierten Facharbeitskräften und spiegelte eine Kulturidentität mit den USA wider, die der Kulturpolitik der KMT - der Ausbildung von loyalen Staatsbürgern, genauer Parteigängern - widersprach. ${ }^{428}$ Um dieser unerwünschten Wirkung durch

\footnotetext{
426 Von 1952 bis 1968 wurde Taiwan auch in die Austauschprogramme des „Smith-Mundt Act“ (1952 bis 1958) und anschließend des „Fulbright Act“ (1958 bis 1968) mit einbezogen. Anschließend sollten sie ihren Landsleuten über die Kultur in den USA berichten. Im Gegenzug besuchten US-amerikanische Fachleute sowie Sportler, Musiker usw. Taiwan, um die taiwanesische Kultur kennenzulernen. Vgl. Chao Ena 2001, S. 87-91 und 97-114.

${ }^{427}$ Von 1950 bis 1989 blieben 80 bis 90\% der taiwanesischen Auslandsstudenten nach ihrem Studium in den USA. Ein großer Teil der zurückgekehrten Studenten arbeitete in den Mittel- und Hochschulen sowie Forschungsinstituten und administrativen Institutionen. Vgl. Lin Hsin-fa 1996, S. 150f. und Chung Ching-han 1988, S. 207-224.

428 Vgl. Neil H. Jacoby 1966, S. 165 und Chen Tian-xi, Yu Hua-mu und Chang Pi-chi 1983, S. 105-107.
} 
die „Zusammenarbeit“ - in Wirklichkeit war es eine Abhängigkeit - mit US-amerikanischen Wissenschaftsgesellschaften entgegenzutreten, erstellte die KMTRegierung eigene Programme, z.B. die „Bewegung zur Bewahrung und Restauration der chinesischen Kultur“. ${ }^{429}$ Zudem wurde mit der Einrichtung einer ständigen Kommission (1969) die Entwicklung der naturwissenschaftlichen Forschung und Ausbildung stärker unter staatliche Kontrolle gebracht und zielgerichtet gefördert. Außerdem wurde der ,gezielte Plan zur Ausbildung von Arbeits- und Forschungskräften“ mit der gesamten Entwicklung des Landes koordiniert, um die Forschung im Dienst der wirtschaftlichen Entwicklung zu fördern und günstige Arbeitsbedingungen für die qualifizierten Facharbeitskräfte zu schaffen.

\subsubsection{Der „Direct Council for Development of Science“}

1967 wurde ein neues Gremium, der „Nationale Sicherheitsrat“, in Taiwan gegründet, der den US-amerikanischen „National Security Council“ zum Vorbild hatte, um den Präsidenten in allen Fragen der Verteidigungsstrategie, der Umstrukturierung der Gesellschaft und des Wirtschaftsaufbaus $\mathrm{zu}$ beraten. Der Rat wurden in vier Abteilungen gegliedert: je eine für Aufbaufragen, für Umstrukturierung der Gesellschaft, für Sicherheitsfragen und für die Steuerung des Wissenschaftsbereichs, Letztere wurde 1969 als „Direct Council for Development of Science“ aufgebaut und Wu Ta-you als Leiter berufen. Die Kommission hatte die Aufgabe, Richtlinien für die Politik und den Haushaltsplan für die Gesamtentwicklung von Technologie und Wissenschaft $\mathrm{zu}$ erstellen und die Durchführung in den entsprechenden Regierungsstellen zu beaufsichtigen. ${ }^{430}$ Damit richtete sich die Entwicklung der naturwissenschaftlichen Bildung und Forschung auf Taiwan nicht mehr nur am Ideal

\footnotetext{
${ }^{429}$ Vgl. 6.2.4. in dieser Arbeit.

430 Vgl. Bildungsministerium (Hrsg.) 1974, S. 28.
} 
der emigrierten Wissenschaftler aus, die eine „Autonomie der Forschung“ und „Verbreitung der naturwissenschaftlichen Kenntnisse zur Verankerung der Forschung in der Heimat" gefordert hatten, sondern sollte einen Beitrag für die gesamte Entwicklung des Landes leisten, vor allem für die Ausbildung der Forschungs- und Lehrkräfte in den Hochschulen. ${ }^{431}$

Die erste Aufgabe dieser Kommission waren Erstellung der drei Vierjahresplänen, die in einen „Zwölfjährigen Plan zur wissenschaftlichen Entwicklung“ mündeten; von 1969 bis 1981 dienten diese Pläne der Zusammenarbeit unterschiedlicher Ministerien wie Bildung, Wirtschaft, Verkehr und Verteidigung u.a. sowie Kommissionen wie dem „Atomic Energy Council“, dem „Council for International Economic Cooperation and Development“ (CIECD) und der „Sino-American Joint Commission on Rural Reconstruction“. „The National Council on Long-range Science Development“ wurde zum „National Council on Scientific Development"، umorganisiert, der den Auftrag zur Durchführung dies Planes übernahm, welcher durch drei Schwerpunkte gekennzeichnet war:

(1) Verbesserung der Bedingungen für die wissenschaftliche Bildung, Forschung und Ausbildung, damit die technischen Facharbeitskräfte in Koordination mit dem Bedarf beim Aufbau des Landes in unterschiedlichen Bereichen ausgebildet werden konnten, z.B. in Industrie, Verteidigung, Landwirtschaft, Verkehr, Kernkraft, Medizin und öffentlicher Gesundheitspflege. Auch die Lehrkräfte für wissenschaftliche Fächer in unterschiedlichen Stufen und Formen der Schulen sollten einbezogen werden;

(2) Förderung der wissenschaftlichen Forschung in unterschiedlichen Fachgebieten wie Mathematik, Physik, Chemie, Biologie, Erdkunde, Meereswesen, Technologie und Landwirtschaft sowie Ausbildung hoch qualifizierter Forschungskräfte, Doktoren 
und Magister. Damit sollte das Niveau der wissenschaftlichen Entwicklung an die fortschrittlichen Länder angeglichen und die Unabhängigkeit der Wissenschaftsentwicklung erreicht werden;

(3) Betreiben und Förderung der Forschung in angewandten Wissenschaften, vor allem derjenigen, die auf die Volkswirtschaft bezogen sind, um damit die Entwicklung von Industrie und Handel zu beschleunigen. ${ }^{432}$

Diese Pläne wurden auch mit der Politik der „Human Resources“ bzw. den „Plänen zur Ausbildung von Arbeitskräften“, die seit Mitte der 1960er-Jahre verfolgt wurden, koordiniert, was zu einer radikalen Änderung in der Struktur des Bildungswesens führte. ${ }^{433}$

Chiangs Regime verfügte über die stärkste Militärmacht des nichtkommunistischen Asiens und stand unter dem Schutz der USA, deren Entwicklungshilfe Taiwan dadurch zugutekam. Dafür sorgten u.a. taiwanesische Wissenschaftler, die im Westen studiert hatten und sich nach ihrer Rückkehr um den Aufbau der beruflichen, naturwissenschaftlichen und technischen Bildung im Land bemühten. Mithilfe von US-Entwicklungshilfe und -Förderprogrammen wurde ein praxisbezogenes Berufsausbildungsmodell geschaffen und Lehrkräfte und Forschungskräfte ausgebildet, was eine eigenständige Weiterentwicklung nach Abbruch der US-Entwicklungshilfe im Jahr 1966 ermöglichte.

\footnotetext{
432 Vgl. Bildungsministerium 1974, S.917 und $922 \mathrm{f}$.

433 Vgl. J. Megan Greene 2007, S.128-153.
} 


\section{Der Umbau des Bildungssystems von 1945 bis 1987}

Das während der japanischen Kolonialzeit entstandene Konzept eines modernen Bildungswesens von der allgemeinen Elementarbildung bis hin zum Universitätsstudium wurde unter Berücksichtigung der chinesischen Schuledikte von 1922, das eine Annähung an das USA-Bildungsmodell bedeutete, und 1932/33, das unter Einfluss des Reformberichts der Bildungskommission des Völkerbundes entstand, zur Grundlage des von der KMT-Regierung installierten neuen Bildungssystems in Taiwan. Das unten abgebildete Schema zeigt den Aufbau:

\begin{tabular}{|c|c|c|c|c|c|c|c|c|c|}
\hline Alter & Schuljahr & \multicolumn{8}{|c|}{ Abbildung 2. Bildungssystem von 1945 bis 1967} \\
\hline 21 & 16 & \multirow{4}{*}{ Tertiärstufe } & & \multirow{4}{*}{ Bachelorstudium } & \multirow{4}{*}{$\mid \begin{array}{l}\text { Fachhochsc } \\
\text { hule } \\
\text { (zwei bis } \\
\text { vier Jahre) }\end{array}$} & \multirow{4}{*}{$\begin{array}{l}\text { Fachschule } \\
\text { (zweir bis } \\
\text { drei Jahre) }\end{array}$} & & & \multirow{5}{*}{ Förderkurse } \\
\hline 20 & 15 & & & & & & & & \\
\hline 19 & 14 & & \multirow{5}{*}{$\begin{array}{l}\text { Pädagogische } \\
\text { Fachschule }\end{array}$} & & & & & & \\
\hline 18 & 13 & & & & & & & & \\
\hline 17 & 12 & \multirow{3}{*}{$\begin{array}{l}\text { Sekundarstu } \\
\text { fe II }\end{array}$} & & \multirow{3}{*}{\multicolumn{3}{|c|}{ Senior High School }} & \multirow{3}{*}{$\begin{array}{l}\text { Senior } \\
\text { Vocational } \\
\text { High School }\end{array}$} & & \\
\hline 16 & 11 & & & & & & & \multirow{5}{*}{\begin{tabular}{|l|} 
Fünfjähri \\
ge \\
Berufssc \\
hule
\end{tabular}} & \multirow{5}{*}{ Förderkurse } \\
\hline 15 & 10 & & & & & & & & \\
\hline 14 & 9 & \multirow{3}{*}{$\begin{array}{l}\text { Sekundarstu } \\
\text { fe I }\end{array}$} & \multirow{3}{*}{\multicolumn{2}{|c|}{ Junior High School }} & \multirow{3}{*}{\multicolumn{3}{|c|}{$\begin{array}{l}\text { Junior Vocational } \\
\text { High School }\end{array}$}} & & \\
\hline 13 & 8 & & & & & & & & \\
\hline 12 & 7 & & & & & & & & \\
\hline 11 & 6 & \multirow{6}{*}{ Primarstufe } & \multirow{6}{*}{\multicolumn{2}{|c|}{ Grundschule }} & & & & & \multirow{6}{*}{ Förderschule } \\
\hline 10 & 5 & & & & & & & & \\
\hline 9 & 4 & & & & & & & & \\
\hline 8 & 3 & & & & & & & & \\
\hline 7 & 2 & & & & & & & & \\
\hline 6 & 1 & & & & & & & & \\
\hline
\end{tabular}

Die Gliederung berücksichtigt die physische und psychische Entwicklung des Menschen, nämlich die Kindheitsphase von 6 bis 12 Jahren, die Pubertätsphase von 12 bis 18 Jahren sowie die Jugendzeit von 18 bis 24 Jahren. Somit dauerte die Grundschulzeit sechs Jahre, die High School wurde in eine dreijährige Junior High School und eine dreijährige Senior High School unterteilt. In der Tertiärstufe dauerten 
die Bachelorstudien an der Universität ca. vier Jahre, während die darauf aufbauende Graduiertenschule als höchste Stufe des Systems keine festgelegte Studienzeit hatte.

\subsection{Umbau der Elementarbildung}

Während die Schuledikte von 1912/13 und 1922 noch eine vierjährige Schulpflicht vorschrieben, empfahlen die Berater der Bildungskommission des Völkerbunds 1932 eine Verlängerung auf sechs Jahre. Zudem sollte der Einschulungstest abgeschafft und die Schulgebühren aufgehoben werden, damit die Chancengleichheit gesichert sei. ${ }^{434}$ Das Einschulungsalter von sieben Jahren sollte aus dem europäischen Schulsystem übernommen werden, zwar konnten hier Ausnahmen gemacht werden, aber es sollte nicht unter sechs Jahren liegen. Die vierjährige untere und die zweijährige höhere Grundschule sollten zur integrierten sechsjährigen Grundschule umgebaut werden, damit die Kinder eine kontinuierliche Lernumgebung erhielten. Um die Kreativität und die Ausrichtung der Schüler auf die Lebenswirklichkeit zu fördern, sollten die Lehrinhalte lebensnah und national bezogen gestaltet werden. ${ }^{435}$ Diese in die Verfassung der Republik China im Jahr 1947 auf dem chinesischen Festland aufgezogen Vorschläge wurden nun beim Aufbau des taiwanesischen Bildungswesens übertragen. ${ }^{436}$

\subsection{Umbau des Bildungswesens: sekundäre und tertiäre Stufe}

Die KMT-Regierung verzichtete aufgrund der Vorschläge der Bildungskommission auf die Form der Gesamtschule, stattdessen standen den Schülern drei unterschiedliche, voneinander getrennte Bildungswege offen:

\footnotetext{
${ }^{434}$ Vgl. Die Bildungskommission des Völkerbunds 1963, S. 64 und 93-97.

435 Vgl. ebd., S. 65f. und 87f. und S. 97-100.

436 Vgl. Bildungsministerium (Hrsg.) 1984, S. 6.
} 
(1)Der allgemeinbildende Weg führte über fünf- oder vierjährige Mittelschulen zu dreijährigen Junior bzw. Senior High Schools. ${ }^{437}$

(2)Der berufsbildende Weg wurde von der ehemals drei- bis fünfjährigen Berufsschule zur dreijährigen Junior bzw. Senior Vocational High School umgebaut. 1948 kam eine aus der japanischen Kolonialzeit stammende Berufsschule hinzu, nämlich die fünfjährige Landwirtschaftsberufsschule für Absolventen der Grundschule. $^{438}$

(3)Zur Tertiärstufe gehörten das Bachelorstudium an Universität und Fachhochschule sowie zwei- bis dreijährige Studiengänge in der Fachschule. Die ehemals dreijährige Ausbildung zum Grundschullehrer wurde ab 1950 in die fünfjährige Pädagogische Fachschule für Absolventen der Sekundarstufe I verlegt. ${ }^{439}$ Grundschullehrer sollten mindestens die Junior High School abgeschlossen haben. ${ }^{440}$

\subsection{Der Erweiterungsprozess des Bildungssystems von 1945 bis 1987}

Der Erweiterungsprozess des Bildungssystems nach 1945 kann in drei Phasen eingeteilt werden: 1 . von der Rückgabe Taiwans an die chinesische Regierung bis zum Beginn des KMT-Regimes (1949), 2. bis zur Einführung der neunjährigen Schulpflicht (1967) und schließlich bis zur Aufhebung des Kriegsrechts (1987).

\subsubsection{Erste Phase: von 1945 bis 1949}

Nach Einführung der sechsjährigen Schulpflicht (1943) betrug die relative Schulbesuchsrate der Schulkinder im Jahr 1944 71,31\%. Von 1945 bis 1949 änderte sich die Zahl der Grundschüler (Tabelle 4) nicht wesentlich, jedoch nahm die Anzahl

\footnotetext{
437 Vgl. Lai An-ming 1996, S. 139-145.

438 Vgl. Shih Ming-fa 1996, S. 73.

439 Vgl. Bildungsministerium (Hrsg.)1974, S. 531.

440 Vgl. Die Bildungskommission des Völkerbunds 1963, S. 68 und 124-132.
} 
der Schüler in der Sekundarstufe enorm zu: In der Junior High School erhöhte sich die Zahl um das 1,7-fache: von 36.088 auf 60.713; in den Junior Vocational High Schools änderte sich nicht viel, doch in den Senior High Schools und in den Senior Vocational High Schools ist ein noch größerer Anstieg dokumentiert: in Ersteren von 4075 im Jahr 1945 auf 15.667 im Jahr 1949, in Zweiteren von 2444 auf 9500 (Tabelle 5). Während der japanischen Besatzung gab es nur drei der dreijährigen Ausbildungsstätten für Lehrkräfte im Elementarbereich. Die Zahl der Schüler in den fünfjährigen Pädagogischen Fachschulen nahm von 2458 im Jahr 1946 auf 4501 im Jahr 1949 rasch zu.

Durch die Erweiterung und den Abzug der Japaner, welche die meisten Lehrer gestellt hatten, litten die Schulen nun akut an Lehrkraftmangel. ${ }^{441}$ Neben der Rekrutierung von Lehrkräften vom chinesischen Festland wurde 1946 eine neue Pädagogische Fachschule in Taipei zur Ausbildung von Lehrkräften der Sekundarstufe eingerichtet. Zudem wurde die Lehrerausbildung abgekürzt. Die Zahl der Studenten an der Universität wuchs von 2983 im Jahr 1946 auf 4937 im Jahr 1949, in den Fachschulen von 79 im Jahr 1948 auf 969 im Jahr 1949 (Tabelle 5). Die Tertiärstufe wurde in dieser Phase noch nicht erweitert, es wurden nur die bestehenden drei Fachschulen zu Fachhochschulen umgestaltet und die „Siebte Universität des japanischen Kaiserreiches“ zur Staatlichen Universität Taiwan umgewandelt.

Die Erweiterung des Bildungsangebotes vor allem in der Sekundar- und Tertiärstufe hatte folgende Gründe: Während der Kolonialzeit war der Bildungswille der

\footnotetext{
${ }^{441}$ Unter der japanischen Besatzungszeit hatten die Japaner die meisten Lehrerstellen inne; 80\% der Lehrer an den Elementarschulen, gut $90 \%$ in der Mittelschule und fast alle Lehrer in der Hochschule. Vgl. Bildungsbehörde unter den Verwaltungsbehörden des Gouvernements der Provinz Taiwan (Hrsg.) 1955, S. 4.
} 
Taiwanesen durch die Ausweitung der elementaren Bildung gestärkt, jedoch in Richtung höherer Bildung stark unterdrückt worden. Letztere blieb der herrschenden Schicht, also den Japanern oder den privilegierten Schichten der Taiwanesen, vorbehalten. Zugleich wurde eine Verbindung zwischen Ausbildungsstand und öffentlichen Amtstellen sowie Facharbeiterpositionen aufgebaut - z. B. durften Absolventen der „öffentlichen Schulen“ als Angestellte in japanischen Behörden oder als Lehrer oder Dolmetscher tätig sein. ${ }^{442}$ Nach Einführung der sechsjährigen Schulpflicht erhofften sich die Absolventen aus der Grundschule nun Chancen auf eine weiterführende Bildung. ${ }^{443}$ Nach Aufhebung der Separationsmaßnahmen genossen schließlich alle Taiwanesen das gleiche Recht auf Bildung, weshalb sich viele Jugendliche wünschten, dass ihnen der soziale Aufstieg durch schulische Bildung ermöglicht würde. Hinzu kam, dass zu dieser Zeit immer mehr junge Flüchtlinge vom chinesischen Festland eintrafen, die sich für die sekundäre und tertiäre Stufe anmeldeten.

\subsubsection{Zweite Phase: von 1950 bis 1967}

Die Bevölkerung der Insel Taiwan erhöhte sich von ca. 6,090 Millionen im Jahr 1946 auf 8,128 Millionen im Jahr 1952. In den Jahren 1949 und 1950 waren etwa 444.000 Flüchtlinge aus China gekommen. ${ }^{444}$ Zudem lag die Geburtenrate in den 1950er Jahren bei gut 4,5 \% pro Jahr und in den 1960er Jahren bei 3,5\% pro Jahr, ${ }^{445}$ hinzu kamen die verbesserten medizinischen Bedingungen. Die Bevölkerung nahm

\footnotetext{
${ }^{442}$ Vgl. Hsu Pei-hsien 2001, S. 59.

443 Vgl. Hsu Nan-hao 1984, S. 84 und ders. 1996, S. $219 f$.

444 Die Anzahl der Flüchtlinge basiert auf einer Vermutung. Es gab unterschiedliche Berechnungsbasen unter den Wissenschaftlern, manche gingen von einer Million, andere von etwa zwei Millionen aus. Vgl. Lin Chiou-huang 2006, S. 15-17.

445 1964-69 wurde die erste Geburtenplanung in Taiwan durchgeführt; die Geburtenrate sank dadurch allmählich. Vgl. Chen Chao-nan, Sun Te-hsiung und Li Tung-ming 2003, S. 79-136.
} 
durchschnittlich jedes Jahr um 350.000 bis 400.000 zu. ${ }^{446}$ Der Zuwachs an Schulkindern verlangte nach einer Ausweitung des Bildungsangebots vor allem im Sekundarbereich.

Aufgrund der Zerstörungen im Krieg und der Inflation war jedoch die wirtschaftliche Aktivität in Taiwan auf einen tieferen Stand gesunken als vor dem Zweiten Weltkrieg. Außerdem gab das KMT-Regime mehr als die Hälfte des Staatshaushalts für die Verstärkung der Streitkräfte aus, dagegen standen nur gut $13 \%$ für den Sektor Erziehung, Wissenschaft und Kultur bereit. ${ }^{447}$ Da das KMT-Regime aber erkannte, dass die schulische Erziehung zur Integration sowie zur Vermittlung politischer Instruktionen im geistigen Kampf gegen die Kommunisten beitragen konnte, und zudem Arbeitskräfte für Landwirtschaft und Infrastruktur ausgebildet werden mussten, förderte die Regierung von 1950 bis 1967 die Grundschulbildung, später auch den Sekundarbereich. Zugleich begann sie mit dem Aufbau der Berufsausbildung sowie der Hochschulen.

\subsubsection{Erweiterung der Elementar- und Sekundarstufe}

Tabelle 6 zeigt, dass es im Jahr 19501231 Grundschulen und 16.856 Klassen mit 906.950 Schülern gab und eine relative Schulbesuchsrate von $79,98 \%$ bei den Schulkindern im Alter von 6 bis 11 Jahren erzielt wurde. Im Jahr 1960 lag die relative Schulbesuchsrate bei $95,59 \%$, d.h. fast alle Kinder im Schulalter waren eingeschult. Die Zahlen der Schüler - 1.888.783 - und der Schulklassen - 35.944 - hatten sich im Vergleich zu 1950 verdoppelt, die Zahl der Schulen hingegen -1843 - hatte nur um 612 bzw. ein Drittel zugenommen. Bis 1967 erhöhte sich der Anteil der Schüler im

\footnotetext{
446 Vgl. CEPD (Hrsg.) 2009, S. 25.

447 Vgl. ders., S. 52.
} 
Alter von sechs bis elf Jahren insgesamt auf 97,52\%, die Zahl der Grundschulen auf 2.208. In 45.171 Klassen wurden 2.348.218 Schüler unterrichtet. Mit der Verbreitung der elementaren Bildung war die Zahl der Lese- und Schreibunkundigen sehr stark rückläufig: Lag die Analphabetenquote für die Bevölkerung über 15 Jahre im Jahr 1951 noch bei $43,43 \%$, so verringerte sie sich bis 1966 auf $23,16 \% .^{448}$

Der massive Zuwachs an Schulkindern und die knappe Finanzförderung stellte jedoch in Anbetracht des Ziels „Bildung für alle“ eine große Herausforderung für die KMT-Regierung dar; zwar versuchte man allen Kindern den Schulbesuch zu ermöglichen, doch schmälerte die rasch wachsende Zahl an Schulkindern die schulische Qualität in den Grundschulen erheblich und forderte zugleich eine Erhöhung der Schulplätze in der Sekundarstufe. Die durchschnittliche Klassenanzahl je Schule betrug 1950 13,69, 196019,50 und 1967 20,46. Die durchschnittliche Schülerzahl je Klasse betrug 1950 53,81, 1960 52,55 und 1967 51,99 (Tabelle 6). In den Großstädten gab es sogar Schulen mit mehr als 100 Klassen und 70 bis80 Schülern pro Klasse. Wegen mangelnder Schulräume fand der Unterricht eines Jahrgangs in zwei Schichten statt, am Vormittag und am Nachmittag. ${ }^{449}$

Die KMT-Regierung versuchte das Problem durch die Errichtung von Junior High Schools zu lösen. Laut Statistik (Tabelle 7) durften jedoch im Jahr 1950 nur 31,78\% der Grundschulabsolventen in die Sekundarstufe I wechseln, also zur Junior High School oder Junior Vocational High School. Bis 1967, kurz vor der Verlängerung der Schulpflichtzeit auf neun Jahre, wechselten $63,66 \%$ in die Sekundarstufe I. Zwischen 1950 und 1967 erhöhte sich die Anzahl der Junior High Schools von 66 auf 288,

\footnotetext{
448 Vgl. Hwang Tsung-mu 2007, S. 32.

449 Vgl. Liu chen 1979, S. $246 f$.
} 
deren Schüleranzahlen stiegen drastisch, um 438.761, an: von 61.082 auf 499.843. Die durchschnittliche Anzahl der Klassen in einer Junior High School lag 1950 bei 20,03 und 1967 bei 32,39, die durchschnittliche Klassenstärke betrug 1950 46,20 Schüler und 1967 53,59 Schüler je Klasse.

Mit Ausweitung der Junior High School konnte zwar der Andrang auf die Sekundarstufe I befriedigt werden, nicht jedoch auf höhere Bildung. Ein weiteres Problem blieb die massive Arbeitslosigkeit der Jugendlichen. Im Jahr 1950 durfte gut die Hälfte, 51,15\%, der Absolventen die Senior High School, Senior Vocational High School oder eine Fachschule besuchen. Im Jahr 1967 erhielten 77,97\% die Chance auf eine weitere Schulbildung in der Sekundarstufe II (Tabelle 7a). Die Anzahl der Schulen und Schüler in den Senior High Schools stieg ebenfalls: 1950 bestanden 62 Schulen mit 18.866 Schülern und 1967170 Schulen mit 140.604 Schülern. Bei den Senior Vocational High Schools waren es 1950 77 Schulen mit 11.226 Schülern und 1967136 Schulen mit 94.547 Schülern. Das Verhältnis der Schülerzahlen zwischen Senior High School und Senior Vocational High School blieb von 1950 (62,7 zu 37,3) bis $1967(59,8 \mathrm{zu}$ 40,2) relativ unverändert (Tabelle 8).

\subsubsection{Erweiterung differenzierter Ausbildungsgänge}

Durch Unterstützung der US-Entwicklungshilfe wurden im Laufe der 1950er-Jahre mehr gewerbliche und landwirtschaftliche Schulen gegründet. 1952 gab es 77 Vocational High Schools (44 Junior Vocational High Schools, 1 Senior Vocational High School, 32 Junior und Senior Vocational High Schools): 38 landwirtschaftliche Schulen, 14 gewerbliche Schulen, 15 Handelsschulen, 3 Schulen für Meereswesen, 2 Schulen für Medizin und Krankenpflege und 5 Schulen für Hauswirtschaft. Die Junior 
Vocational High School besuchten insgesamt 25.860 Schüler, die Senior Vocational High School 14.232 Schüler. Bis 1967 belief sich die Anzahl der Vocational High Schools auf 136, wobei sich die Anzahl der Schüler in den Junior Vocational High Schools auf 48.749 Schüler verdoppelte und in den Senior Vocational High Schools um das 6,6-fache zunahm, auf 94.547 (Tabelle 7 und 8). Die Tabellen 9 und 9a zeigen, dass sich die Anzahl der Schüler in den gewerblichen und landwirtschaftlichen Bereichen durch die Unterstützung der US-Entwicklungshilfe erhöhte, jedoch nahm der Anteil der Schüler im landwirtschaftlichen Bereich, gemessen an der gesamten Schülerzahl, in den Vocational High Schools von 36,75\% auf 18,73\% deutlich ab. Hingegen stieg die Anzahl der Schüler im Handelsbereich drastisch an, von 36.612 im Jahr 1960 auf 75.178 im Jahr 1967.

\subsubsection{Erweiterung der Tertiärstufe}

1948 war der tertiäre Bildungsbereich, mit Ausnahme von militärischen Hochschulen, noch wenig entwickelt. Die KMT-Regierung war nun dazu gezwungen, den Bereich zu erweitern, um einer großen Anzahl an Schülern und Studenten die Fortbildung zu ermöglichen und Stellen für Wissenschaftler unter den Flüchtlingen zu schaffen. ${ }^{450}$

\subsubsection{Ausbau von Universitäten und Fachhochschulen}

Trotz knapper staatlicher Förderungsmittel wurden folgende Maßnahmen zum Ausbau der höheren Bildung ergriffen:

(1) Erweiterung der „National Taiwan University“ und Umwandlung der Fachhochschulen in Universitäten, so wurden mit der Unterstützung durch die US-Entwicklungshilfe die technische Fachschule in Tainan zur „National Cheng

\footnotetext{
450 Vgl. Bildungsministerium (Hrsg.) 1957, S. 481-490 und 499-505.
} 
Kung University” (1956) und die Pädagogische Fachschule in Taipei zur staatlichen Universität für Pädagogik, zur „National Taiwan Normal University“ (1955); ${ }^{451}$

(2) erneute Gründung von bereits auf dem chinesischen Festland existierenden staatlichen Universitäten, der „National Chen Chi University” (1954) zur Ausbildung von Verwaltungsbeamten ${ }^{452}$ und der „National Tsing Hua University” (1956) sowie der „National Chiao Tung University” (1958) für naturwissenschaftliche Forschung und Ausbildung. ${ }^{453}$

(3) Wiederaufbau und Neuerrichtung von Universitäten durch Missionare und private Träger, z.B. der von der Steyler Mission und dem Jesuitenorden gemeinsam errichteten Fu Ren University (1960). ${ }^{454}$

Die Hochschulen wurden größtenteils von Festlandschinesen errichtet, nur zwei davon (die medizinischen Fachhochschulen in Kaohsiung und Taipei) von Taiwanesen. Zugleich wurden die meisten Lehrstellen von emigrierten chinesischen Wissenschaftlern übernommen. Emigrierte chinesische Jugendliche sowie Soldaten bekamen hier die Gelegenheit zur Weiterbildung. ${ }^{455}$ Die Ausbildung neuer Lehr- und Forschungskräfte wurde erst ab Mitte der 1960er-Jahre mit Abschluss des Abkommens für wissenschaftliche Zusammenarbeit zwischen Taiwan und den USA und einer Reihe von Austauschprogrammen möglich.

\footnotetext{
451 Vgl. Lin Chian-fu 1993, S. 163-171 und Bildungsministerium (Hrsg.) 1984, S. 977f. und 982.

452 Diese Universität behält eine Sonderstellung in der chinesischen bzw. taiwanesischen Bildungsgeschichte, weil sie aus der damaligen Parteihochschule (erste Gründung 1928) zur Ausbildung der Verwaltungskräfte der KMT hervorging. 1945 wurde sie in Cheng Chi (wörtlich: Politik-) Universität umbenannt und 1949 im Zuge der Wirren der kommunistischen Revolution aufgelöst. Erst im Jahre 1954 wurde sie in Taipei wieder eingerichtet, denn die KMT benötigte leitendes pädagogisches und administratives Personal. Vgl. Chang Chi-yun 1982 (b), S. 464-467.

453 Vgl. Bildungsministerium (Hrsg.) 1984, S. 978 und 981-984.

454 Vgl. ebd., S. 985-993 und 996-1001.

455 Eine Untersuchung an der staatlichen Universität Taiwan im Zeitraum von 1954 bis 2000 (Luoh 2002) zeigte, dass im Zeitraum von 1954 bis 1980 ca. 40\% der gesamten Studenten vom chinesischen Festland stammten. Vgl. Luoh Ming-ching 2002, S. 113-147.
} 


\subsubsection{Gründung von Fachschulen}

Ab 1951 wurden neue Fachschulen gegründet und in den 1960er-Jahren ein Teil der Senior Vocational High Schools zu Fachschulen umgeformt. Zudem wurden Fachschulen mit zwei- bis dreijährigem Studiengang für Absolventen der Sekundarstufe II in den Fachbereichen Agrarwirtschaft, Medizin und Pflege, Fremdsprache, Technik, Hauswirtschaft, Journalismus, Kunst, Handelswesen, Sport, Verwaltungswesen, Fischerei und Meereswesen sowie Militär und Polizei installiert. $^{456}$

Tabelle 10 zeigt, dass es im Jahr 1950 nur wenige Institutionen der höheren Bildung gab: 1 Universität und 3 Fachhochschulen mit 5374 Studenten sowie 3 Fachschulen mit 1286 Studenten. 1967 bestanden 9 Universitäten und 13 Fachhochschulen mit 73.881 Studenten sowie 57 Fachschulen mit 63.411 Studenten, $40.372 \mathrm{im}$ zweijährigen und 23.039 im dreijährigen Studiengang.

\subsubsection{Schaffung von Abendkursen und Förderkursen}

Immer mehr Absolventen der Sekundarstufe II nahmen an der Aufnahmeprüfung zur Hochschule teil, jedoch konnte die Regierung in der kurzen Zeit nicht entsprechend viele Studienplätze schaffen. Daher fanden zahlreiche Absolventen vor allem nach der Senior High School weder einen Studien- noch einen Arbeitsplatz. Ab 1966 wurde ein alternativer Studiengang, ein Abendkurs an Universitäten, Fachhochschulen und Fachschulen, installiert. Damit erwuchs eine Möglichkeit der beruflichen Weiterbildung, einerseits für Schulabsolventen, andererseits für Arbeitnehmer. ${ }^{457}$ Zudem wurden Förderkurse und Lehrgänge für von der Schule abgehende

\footnotetext{
456 Vgl. Bildungsministerium (Hrsg.) 1974, S. 656f.

457 Vgl. ebd. S. 758-761.
} 
Erwachsene der Senior High Schools, Junior High Schools und Grundschulen eingerichtet.

In dieser Phase bemühte sich die Regierung, den massiven Bildungsbedarf der Bevölkerung nach dem Prinzip der Chancengleichheit sowohl für die weibliche als auch die männliche Bevölkerung sowie für unterschiedliche Sozialgruppen abzudecken. Dabei wurden jedoch der Bedarf des Arbeitsmarktes und der Zusammenhang zwischen Bildung und der wirtschaftlichen und sozialen Entwicklung kaum berücksichtigt. Die Erweiterung des Bildungsangebots richtete sich vor allem in der sekundären und tertiären Stufe immer noch auf Allgemeinbildung.

\subsection{Die Konzepte „Human Capital“ und „Human Resources“}

Die Konkretisierung des auf den Beruf ausgerichteten Bildungswesens gewann in den 1960er-Jahren neue Impulse durch die Fachdisziplin „Bildungsökonomie“. Die These, dass höhere Bildung und Qualifikation der Arbeitskräfte einen höheren Beitrag zur Produktion leisten, wurde bereits während der industriellen Revolution im 18. Jahrhundert durch die britischen Ökonomen Adam Smith (1723-1790) und David Ricardo (1772-1823) aufgestellt. ${ }^{458}$ Nach dem Sputnik-Schock rückte sie erneut in den Vordergrund wissenschaftlicher Auseinandersetzungen. 1957 veröffentlichten bedeutende US-amerikanische Ökonomen wie Theodore W. Schultz, Gary Becker und Edward F. Denison ${ }^{459}$ eine Analyse der ökonomischen Dimension von Bildungssystemen, -institutionen und -prozessen und bezogen dabei Kosten und

${ }^{458}$ Vgl. Harry Maier 1994, S. 1-9.

459 Theodore W. Schultz und Edward F. Denison zeigten den Zusammenhang von Bildungsniveau der Bevölkerung mit wirtschaftlichem Wohlstand am Beispiel der USA auf. Im Zeitraum von 1929-1953 sei möglicherweise nur ein Fünftel des Produktivitätszuwachses auf die Verbesserung der Produktionsmittel zurückzuführen, während die restlichen vier Fünftel verbesserten Arbeitsmethoden, besserem Management und Höherqualifizierung der Arbeitskräfte zuzuschreiben seien. Vgl. Theodore W. Schultz 1961 und Edward F. Denison 1962. 
Nutzen der Bildungsausgaben auf wachstumstheoretische Fragestellungen, etwa den Zusammenhang zwischen staatlichen Investitionen für Bildung und Forschung und der gesamtwirtschaftlichen Entwicklung eines Landes bzw. zwischen Bildung und Forschung als qualitativen Produktionsfaktoren mit quantitativen Produktionsfaktoren wie Geld und Arbeit. ${ }^{460}$ Das Konzept „Human Capital“ geht davon aus, dass die im Bildungssystem erworbenen Kenntnisse, Fähigkeiten und Fertigkeiten nicht nur zur Verbesserung der potenziellen Erwerbstätigkeit führen, sondern auch zur Erhöhung der sozialen Stellung des Einzelnen sowie des kulturellen Entwicklungsniveaus der gesamten Gesellschaft beitragen können. Der Ansatz verwies erneut auf die Bedeutung der „Human Resources“ für das langfristige Wachstum einer Volkswirtschaft. ${ }^{461}$

Gemäß „Human Capital“ und „Human Resources“ eröffnete eine Bildungsexpansion die Möglichkeit, den Bedarf einer modernen Industriegesellschaft an qualifizierten Arbeitskräften für die wirtschaftliche Entwicklung zu decken und durch eine Anhebung des Bildungsniveaus rationales Verhalten angesichts eines komplexer werdenden Gesellschaftssystems zu verbessern sowie die Lernenden stärker gemäß ihren Fähigkeiten und Neigungen $\mathrm{zu}$ fördern. Um die optimale Nutzung aller „Begabungsreserven“ der Bürger in Hinblick auf den Bedarf an gut qualifizierten Fachkräften zu sichern und dadurch eine anhaltend günstige wirtschaftliche Entwicklung zu erlangen, regten viele Länder, die bereits die Schulpflicht für die allgemeine Elementarbildung eingeführt hatten, an, ihre öffentlichen Budgets zur Erweiterung des Bildungsangebotes in den sekundären und tertiären Bildungs-

\footnotetext{
${ }^{460}$ Bildungsökonomen wie Frederick Harbison und C. A. Meyers (1964) hatten die Beschulungsquote der Bevölkerung im Alter von fünfzehn bis neunzehn Jahren als wichtigen Indikator für die wirtschaftliche Entwicklung eines Landes gedeutet.

${ }^{461}$ Vgl. Gary Becker 1993 und Theodor W. Schultz 1961.
} 
bereichen zu nutzen. Mehr Bildungsplätze zu schaffen und zugleich damit bisherige Barrieren für bildungsfernere bzw. benachteiligte Gruppen aufzuheben, außerdem eine entsprechende Unterstützung für diese Gruppen aufzubauen wie in Deutschland in den 1970er Jahren wurde zum Ziel der Bildungspolitik. ${ }^{462}$

Diese Konzepte wurden auch von internationalen Hilfsorganisationen aufgegriffen. So hatte die Weltbank um 1961 erkannt, dass die Darlehensnehmerländer wegen des Mangels an qualifizierten Arbeitskräften in ihrer Wirtschaftsentwicklung verzögert waren und deswegen ihre Schulden nicht rechtzeitig zurückzahlen konnten. Daraufhin konzipierte sie ab 1963 eine Reihe von Darlehensprojekten für die Bildungsentwicklung, vor allem im Bereich der beruflichen Ausbildung. ${ }^{463}$ Auch die UNESCO leitete internationale Erziehungskonferenzen zur Erstellung von Bildungsprogrammen in den Entwicklungsländern ein. ${ }^{464}$ Seit den 1960er-Jahren wurden „Human Capital“ und „Human Resources“ in vielen Ländern Ausgangspunkt

\footnotetext{
462 Diese Bildungsdiskussion wurde im Zusammenhang mit der politischen Aufbruchsituation mit sinkenden Wachstumsraten der deutschen Volkswirtschaft, mit internationalen Bildungsvergleichen sowie Forderungen nach steigender Produktivität und Qualifikation geführt. Vor allem die Schriften von Georg Picht „Die deutsche Bildungskatastrophe“ (1964) und Ralf Dahrendorfs „Bildung ist Bürgerrecht" (1965) brachten die Bildungsprobleme in das Bewusstsein der Öffentlichkeit. Georg Picht wies auf den Lehrermangel, zu geringe Abiturientenzahlen und einen Mangel an Klassenräumen hin. Der finanzielle Aufwand für Bildungsausgaben war in Deutschland relativ gering, verglichen mit anderen Ländern. Ralf Dahrendorf betonte die Abhängigkeit der Bildungschancen des Kindes von seiner sozialen Herkunft. Er sah die Ursache beim Übergang zur weiterführenden Schule in den Einstellungen der betreffenden benachteiligten Bevölkerungsgruppen. Diese Debatte löste in starkem Maße die Erweiterung der Bildungschancen im sekundären und tertiären Bereich aus. Vgl. Ralf Dahrendorf 1968; Georg Picht 1965; Rainer Geißler 1996, S. 249-274 und ders. 2000.

${ }^{463}$ In der ersten Phase (1963-69) richtete die Weltbank ihre Unterstützung auf Mittelschulen und Berufsausbildung und berücksichtigte kaum die Frage der elementaren Bildung in den Entwicklungsländern; erst ab 1980 wandte sie die Politik der Entwicklungshilfe auf die allgemeine Elementarbildung an. Vgl. Chan Sheng-ju 2000, S. 114-116.

${ }^{464}$ Die internationalen Bildungskonferenzen 1958 in Washington, USA, 1962 in Santiago di Chile und 1966 in Buenos Aires, Argentinien, behandelten die Bildungsentwicklung in Lateinamerika, die Bildungskonferenzen der OECD 1960 in Berlin, Deutschland, und 1961 in Washington, USA, betrafen die Bildungsentwicklung in Europa und Nordamerika. Außerdem richtete die UNESCO Institutionen zur Ausbildung von Fachleuten für die Erstellung und Durchführung von Bildungsplänen ein. Man diskutierte und erstellte 1959/60 in Karachi, Indien, 1962 in Tokio, Japan, und 1965 in Bangkok, Thailand, Gesamtentwicklungspläne für Asien. Taiwan als Mitgliedsland von Weltbank und UNESCO wurde in die Entwicklungspläne einbezogen. Vgl. Kang Tai-kuang (Hrsg.) 1965, S. 27f.
} 
für den Aufbau einer elementaren Bildung mit Schulpflicht oder für die Erweiterung der sekundären und tertiären Bildung. Die quantitative Entwicklung der nationalen Bildungssysteme kommt daher einer „World Educational Revolution“ gleich, wie Meyer u. a. (1977) es bezeichnen. ${ }^{465}$

\subsection{Bildungsökonomie und staatliche Planung}

Mitte der 1960er-Jahre gab die KMT-Regierung ihr Vorhaben eines Krieges gegen die chinesischen Kommunisten auf. Für militärische Ausgaben gedachte Gelder konnten nun in die Bildung fließen. Zudem benötigte Taiwan mit dem Abbruch der US-Entwicklungshilfe 1966 eigenständige Strategien für die weitere Entwicklung des Landes. Vom dritten bis fünften Vierjahresplan (1961-1964, 1965-1968 und 19691972) an bezogen sich die Pläne der Landesentwicklung nicht mehr nur auf die Wirtschaft, sondern auch auf Verkehr, Technologie, Erziehung und den kulturellen und sozialen Aufbau. ${ }^{466}$ Die Wirtschaftspolitik setzte Schwerpunkte auf arbeitsintensive Fertigungsindustrien durch die Übernahme von Produktionstechniken aus Japan und den USA, die Ausweitung des Exports richtete sich auf den US-Markt. So baute Taiwan seine Beziehung zu den Weltmächten auf und wurde schließlich in die

\footnotetext{
465 Vgl. John W. Meyer und Francisco O. Ramirez u. a. 1977, S. 242-258. Im Untersuchungsbericht „The World Educational Revolution, 1950-1970“ (1977) - einer Analyse von UNESCO-Daten zur Beschulungsrate in primären, sekundären und tertiären Bildungsinstitutionen von 117 Ländern im Zeitraum von 1950-1970 - wiesen John W. Meyer und seine Mitarbeiter darauf hin, dass die reicheren Länder die Schulpflicht bereits vor 1950 eingeführt hatten. Die relative Schulbesuchsrate im Primarbereich betrug hier fast $100 \%$. Die Beschulungsrate im Sekundar- und Tertiärbereich betrug 21,3\%. Von 1950 bis 1970 hatten die reicheren Länder ihre sekundäre Bildung enorm erweitert: Die Beschulungsrate in sekundären Bildungsinstitutionen stieg auf $46,45 \%$, in tertiären Bildungseinrichtungen von 2,6\% auf 9,2\%. Die ärmeren Länder hinkten hinterher. Die Beschulungsrate in primären Bildungseinrichtungen betrug in den 1950er-Jahren hier nur 37\%, aber bereits in den 1970er Jahren stieg sie auf 72\%. Die Erweiterung der Bildungsplätze im sekundären und tertiären Bildungsbereich setzte sich fort. Die Beschulungsrate in der sekundären und tertiären Bildung stieg von $5,3 \%$ und $0,6 \%$ auf $17.0 \%$ und 2,6\%. Im Jahr 1985 hatten $80 \%$ aller Länder auf der Erde die Schulpflicht eingeführt. Ende der 1980er-Jahre verbrachte bereits über $90 \%$ der Kinder weltweit ihre Kindheit in der Schule. Im Jahr 2005 betrug die durchschnittliche Beschulungsrate in der Sekundarstufe II, also im Alter von 15 bis 19 Jahren, in den Ländern der OECD 81,5 \%, im tertiären Bereich, im Alter von 20 bis 29 Jahren 24,9\%. Vgl. OECD 2007, S. 322 f.

466 Vgl. ebd. (c) und (d) und ders. 1972.
} 
Weltwirtschaftsgemeinschaft integriert. Im Zuge dessen wurden unter Berücksichtigung des Konzepts „Human Resources“ Pläne zur Ausbildung von Arbeitskräften erstellt. Der zuständige Beamte für die Gestaltung der „Vierjahrespläne zum wirtschaftlichen Aufbau“, Li Kwoh-ting, suchte die Nutzbarmachung bildungsökonomischer Methoden zur Förderung der wirtschaftlichen Entwicklung, wie sie in den 1950er-Jahren mit Unterstützung durch die US-Entwicklungshilfe stattfand, auf das taiwanesische Bildungswesen zu übertragen. Damit sollte ein System zur beruflichen Ausbildung von einfachen Arbeitern bis zu professionellen Fachleuten aufgebaut werden, damit die Mehrheit der Bevölkerung als Arbeitskräfte zur Verfügung standen und sich am wirtschaftlichen Aufbau des Landes beteiligen konnten. ${ }^{467}$ Jedoch fehlte den taiwanesischen Beamten die Erfahrung zur Konkretisierung eines langfristigen Bildungsplans. Denn zur Koordination von Bildungswesen und wirtschaftlichem Aufbau müssen unterschiedliche Fachdisziplinen wie Ökonomie, Soziologie, Politikwissenschaft, Statistik und Anthropologie berücksichtigt werden, was zuvor von wissenschaftlichen Beratern des „Stanford Research Institute“ sowie internationalen Organisationen wie der UNESCO und der Weltbank betreut worden war.

\subsubsection{Der Forschungsbericht des „Stanford Research Institute“}

Im Mai 1961 stellte eine Delegation der Stanford Universität Bildungsminister Huang Ji-lu ${ }^{468}$ anhand des Buches „Toward Strategies of Education“ von William Platt das Konzept der „Human Resources“ vor. ${ }^{469}$ Daraufhin beauftragte Huang das „Stanford

\footnotetext{
467 Vgl. Liu Ke-chih 1995, S. 47.

468 Huang Ji-lu (1899-1985) studierte von 1921 bis 1924 an Universitäten in den USA und Kanada. Seit 1924 übernahm er unterschiedliche Positionen in der KMT und war Abgeordneter. Seit 1949 war er Minister des Inneren (1952-1954), Minister für Prüfung und Auswahl der Beamten (1958-1961), Bildungsminister (1961-1965) und Mitglied des Beraterstabs des Präsidenten (1965-1984).

469 Vgl. Stanford Research Institute in California 1962, S. 1.
} 
Research Institute“ mit einer Analyse. Das Forschungsprojekt beabsichtigte:

(1)die gegenwärtige Entwicklung und mögliche Reformpläne im Bildungswesen zu untersuchen;

(2)den Umfang der gegenwärtigen Arbeitskräftezahlen in unterschiedlichen Sektoren zu schätzen;

(3)den zukünftigen Bedarf an Fach- und angelernten Arbeitskräften für die einzelnen Sektoren gemäß dem dritten „Vierjahresplan für den wirtschaftlichen Aufbau“ einzuschätzen;

(4)die Diskrepanz zwischen benötigten Arbeitskräften und ausgebildeten Schülern zu berechnen;

(5)angemessene Maßnahmen zur Lösung der Diskrepanz zwischen der Ausbildung der Arbeitskräfte und den Erfordernissen der Arbeitswelt zu entwickeln;

(6)Anreize zu konzipieren, mit denen die Berufs- und Fachschulen begabte Schüler gewinnen könnten;

(7)Hilfsmaßnahmen für die Ausgebildeten zu konzipieren, damit sie ihre erlernten Fertigkeiten in den entsprechenden Gewerben umsetzen können;

(8)den Einfluss des Aufnahmeprüfungssystems auf die Bildungsqualität und die langfristige Wirtschaftsentwicklung zu untersuchen. ${ }^{470}$

1962 wurde der Forschungsbericht der kalifornischen Wissenschaftler mit dem Titel „Education and Development - The Role of Educational Planning in the Economic Development of the Republic of China“ veröffentlicht. Die Ergebnisse waren jedoch für aufgrund des Forschungsdesigns eher unergiebig, z.B. bezogen sich die Kategorien der Berufstypen nur auf Verwaltungsbeamte, Techniker, Facharbeiter und angelernte Kräfte; nur ein kleiner Teil der Bevölkerungsschichten war einbezogen, 
und das Datenmaterial war sehr spärlich; dementsprechend waren die Reformvorschläge sehr allgemein gehalten und ließen sich nicht in der Praxis umsetzen. ${ }^{471}$

\subsubsection{Der Forschungsbericht der UNESCO}

In der Zwischenzeit suchten die Beamten auch die Unterstützung von anderen Organisationen. 1963 ermöglichte das Bildungsministerium eine Zusammenarbeit mit der Weltbank und der UNESCO für die Erstellung von Bildungsplänen und den Aufbau von Bildungseinrichtungen. ${ }^{472}$ Eine wissenschaftliche Beratung von Fachleuten wurde erbeten, die einen langfristigen Plan in Anlehnung an die Bildungsökonomie erstellen sollten. Die UNESCO schickte 1963 ihren Mitarbeiter Dr. Martin Stromnes nach Taiwan. ${ }^{473}$ Zusammen mit taiwanesischen Pädagogen erstellte er 1964 anhand des Berichts der Stanford-Studien die Skizze eines langfristigen Bildungsplans, also für eine Generation, von der Grundschule bis zum Ende des Magisterstudiums:

(1)der achtzehnjährige Bildungsplan sollte mit dem wirtschaftlichen Aufbau des Landes koordiniert werden. Auf dieser Basis sollten für den Zeitraum 1964 bis 1982 die benötigten Arbeitskräfte eingeschätzt werden.

(2)in Anbetracht des Bevölkerungswachstums sollten die Gestaltung der Bildungsplätze von der Grundschule bis zur Hochschule und die Ausbildung der Lehrkräfte für die entsprechende Erziehungspopulation geplant werden.

\footnotetext{
471 Vgl. Udo Dörnhaus 1988, S. 75-77; Chen Tien-hsi, Yu Huan-mo und Chang Pi-chi 1983, S. $16 f$. und 31 .

${ }^{472}$ Wegen Schülerzuwachs benötigte die Regierung massive finanzielle Unterstützung zum Ausbau der Bildungseinrichtungen und versuchte einen Kredit bei internationalen Organisationen aufzunehmen. 1964 stellte sie Anträge an die Weltbank. Vgl. Yu Sung-nian 1976, S. 8f., CIECD 1970 und Bildungsministerium (Hrsg.) 1974, S. 644-651.

${ }^{473} \mathrm{Vgl}$. UNESCO und Zentrale Kommission für Zusammenarbeit zwischen schulischer und außerschulischer Ausbildung unter dem Bildungsministerium (Hrsg.) 1964, S. 68-79.
} 
(3)aufgrund des sozialen und wirtschaftlichen Bedarfs im Inland und der internationalen Märkte sollten fünf Sonderpläne zu Beratung, Tests, Erziehung durch Rundfunk und Fernsehen, Förderkursen für Schulabgänger sowie Unterhaltungs- und Kulturerziehung erstellt werden.

(4)auf der Basis der prognostizierten Wirtschaftsentwicklung sollte der benötigte Staatsaufwand zunächst berechnet werden, um dann die Inhalte durch die verfügbaren Staatsausgaben zu bestimmen. Der Bildungsplan sollte in die „Vierjahrespläne zum wirtschaftlichen Aufbau“" integriert werden. ${ }^{474}$

Dr. Stromnes machte dazu folgende Vorschläge:

(1)Ausdehnung der Schulpflichtschulzeit von sechs auf neun Jahre. Die Statistik zeigte, dass es ca. eine Million Analphabeten gab und eine weitere Million junger Menschen zwischen 13 und 18 Jahren weder Schulunterricht noch eine Arbeitsstelle hatten;

(2)Abschaffung der Junior Vocational High Schools. Nur ein kleiner Anteil der Absolventen (1548 von 7350 Schüler des Jahrgangs 1960) stand in einem Beschäftigungsverhältnis. Der Rest besuchte entweder die weiterführende Schule oder war arbeitslos. Die Schulform konnte ihre Aufgabe, die Ausbildung von Arbeitskräften, nicht mehr erfüllen;

(3)Erhöhung der Schülerzahlen an den Senior Vocational High Schools. Um Arbeitskräfte heranzubilden, sollten weniger Schüler allgemeinbildende Schulen besuchen und stattdessen auf berufliche Zweige gelenkt werden. Vor allem der Anteil an Gewerbeschülern sollte an den Senior Vocational High Schools auf $30 \%$ (1962:19\%) erhöht werden;

(4) Erweiterung der Kooperation zwischen Schule und Betrieb;

(5)Beschleunigter Ausbau der fünfjährigen technischen Fachschulen. Letztere wurden

474 Vgl. Kang Tai-kuang und Shen Treng-chi 1965, S. 859-865. 
als „Ersatzhochschulen“ angesehen. Damit sollte der massive Bedarf an Studienplätzen abgedeckt werden;

(6)Einführung von Abendkursen an zwei- und dreijährigen Fachschulen. Bei expandierender Wirtschaftsentwicklung könnte die schulische Ausbildung den Bedarf an hoch qualifizierten Arbeitskräften nicht abdecken. Daher schlug Stromnes die Einrichtung von Abendkursen zur Fortbildung der werktätigen Bevölkerung vor;

(7)Verbesserung der Ausbildung und Arbeitsbedingungen von Lehrern an der beruflichen Schule und Fachschule.

Dr. Stromnes versuchte das Problem der geringen Wertschätzung beruflicher Ausbildung in der taiwanesischen Gesellschaft durch eine Verstärkung der praktischen Ausbildung, durch mehr Forschungsmöglichkeiten und die Verbesserung der Gehälter für Lehrer an beruflichen Schulen und Fachschulen zu beseitigen. ${ }^{475}$ Weiterhin wies er auf die Notwendigkeit einer Institution zur Erstellung von langfristigen Bildungsplänen hin. ${ }^{476}$ Sein Plan führte eine breitere Sicht vor und zeigte machbare Strategien zur Gestaltung der Bildungsplanung auf; Spuren davon finden sich bis in spätere Bildungsreformen.

\subsubsection{Die „Arbeitsgruppe Arbeitskräfteplanung“}

Die internationalen Studien regten taiwanesische Beamte wie den Präsidenten des Exekutivrats, Yen Chia-kan (1905-1993), und hohe Beamte im „Council for United States Aid“ (CUSA) wie Li Kou-ting und Tao Shen-yang ${ }^{477}$ an, sich für Bildungs-

\footnotetext{
475 Vgl. UNESCO und Zentrale Kommission für Zusammenarbeit zwischen schulischer und außerschulischer Ausbildung unter dem Bildungsministerium (Hrsg.) 1964, S. 324-326.

476 Vgl. ebd., S.1.

477 Tao Shen-yang (1919-1969) studierte Technologie an der Technischen Universität in Berlin (1939), war Beamter am CUSA (1959), Sekretär am CIECD (1963-1969) und Wirtschaftsminister (1969). Er galt stets als Förderer der deutsch-taiwanesischen Beziehungen. 
ökonomie zu interessieren. ${ }^{478}$ Im Jahr 1963 wurde eine zentrale eigenständige Planungsinstanz, die „Arbeitsgruppe Arbeitskräfteplanung“ (Manpower Development Committee) innerhalb des „Council for International Economic Cooperation und Development“ (CIECD) unter Leitung von Li Kou-ting eingerichtet. Die Gestaltung des Bildungssystems richtete sich nun am Bedarf von Arbeitskräften im Hinblick auf die Durchsetzung der „Vierjahrespläne zum wirtschaftlichen Aufbau“ und des „Zwölfjahresplans für wissenschaftliche Entwicklung“ (1969-1981) aus. Das Wirtschaftsministerium übernahm somit eine entscheidende Rolle in der Bildungspolitik.

Von 1966 bis 1969 wurden drei „Exportverarbeitungszonen“ (Export Processing Zones) in Kaohsiung und Taichung gegründet, in denen besondere Bedingungen herrschten wie Steuervergünstigungen, billige Arbeitskräfte und Befreiung von Einfuhrzöllen für Investitionen von Ausländern und Überseechinesen in Taiwan, dadurch wurden massiv Arbeitsplätze für die Beschäftigung der ländlichen Bevölkerung geschaffen. ${ }^{479}$ Auf dieser Basis legte 1966 die Arbeitsgruppe den „Ersten Plan zur Ausbildung von Arbeitskräften“, der mit Hilfe US-amerikanischer Berater wie Chester C. Hepler und Alice W. Shurcliff entstanden war, vor; ${ }^{480}$ er orientierte sich an den Forschungsberichten des „Stanford Research Institute“ sowie der UNESCO. Zur Maximierung der Rentabilität suchte er Ausbildung und Qualitätssteigerung bestmöglich am Bedarf des wirtschaftlichen Aufbaus auszurichten, zugleich sollte die Arbeitslosigkeit gemildert werden. Ziel war, den Wohlstand anzuheben und die junge Bevölkerung in differenzierte Bildungszweige und Lehrgänge zu bringen. ${ }^{481}$

\footnotetext{
478 Vgl. Li Kou-ting 1961 und 1962.

479 Vgl. Statistisches Bundesamt Wiesbaden (Hrsg.) 1991, S. 124, und Li Kou-ting 1978, S. 92-100.

480 Vgl. Chen Tien-hsi, Yu Huan-mo und Chang Pi-chi 1983, S.18.

481 Vgl. ebd., S. 18-20.
} 
Aufgrund von Untersuchungen über Bevölkerungsverteilung in Arbeitswelt und Ausbildung wurden kurzfristige und langfristige Programme für die vom Arbeitsmarkt benötige Fachausbildung erstellt. Der erste Plan war unterteilt in je einen kurz-, mittel- und langfristigen Teil, der jeweils einem zwei-, fünf- und zehnjährigen Planungszeitraum entsprach, jedoch sollte er alle zwei Jahre revidiert werden. $^{482}$ Der Fokus lag auf der Altersgruppe der Zwölf- bis Vierzehnjährigen. Zunächst wurden die Änderung der Sekundarstufe I behandelt. Die Bildungsmöglichkeiten in der Sekundarstufe II und Tertiärstufe wurden mit einer Reihe revidierter Pläne ${ }^{483}$ erweitert und verbessert.

Bis zum Beginn der 1970er-Jahre erfuhr die taiwanesische Wirtschaft ein rasches Wachstum durch kapital- und technologieintensive Produktion, industriellen Ausbau und zunehmende weltwirtschaftliche Integration, was oft als Wirtschaftswunder bezeichnet wurde. ${ }^{484}$ Doch wegen der ersten Ölkrise im Jahr 1973 blieb das gesamtwirtschaftliche Wachstum in den Jahren 1974 und 1975 zurïck. Außerdem musste die taiwanesische Regierung nach dem Rückzug aus der UNO ein Scheitern ihrer Außenpolitik hinnehmen. Der 1954 unterzeichnete „Sino-American mutual defense treaty“ wurde wegen des Abbruchs der diplomatischen Beziehung zu den USA im Jahr 1979 annulliert. Taiwan verlor nicht nur die politische, sondern auch die

\footnotetext{
482 Der kurzfristige Teil konnte aufgrund detaillierter Zahlen sofort in die Praxis umgesetzt werden. Hingegen wurde der mittel- und langfristige Teil nur als Perspektive für die weitere Entwicklung verstanden. Diese Form des Berichtes fand man auch in später erstellten Plänen. Vgl. Chen Tien-hsi, Yu Huan-mo und Chang Pi-chi 1983, S. 20-22.

483 „Revidierter Plan zur Ausbildung von Arbeitskräften“ (1968), „Der dritte Plan zur Ausbildung von Arbeitskräften“ (1970), „Der vierte Plan zur Ausbildung von Arbeitskräften“ (1971-1981).

${ }^{484}$ Das Pro-Kopf Einkommen der Bevölkerung in Taiwan betrug von 1951 bis 2008: 1951: 137 USD auf 1961:143 USD, 1971:414 USD, 1981:2.486 USD. Vgl. Directorate General of Budget, Accounting and Statistics under Executive Yuan (Hrsg.) 2008 S.138. Zu Beginn der 1980er-Jahre ließ sich Taiwan international als eines der neuen industriellen Aufsteigerländer (Newly Industrialized Countries) klassifizieren und gehörte mit Hongkong, Südkorea und Singapur zu den ,vier kleinen Tigerstaaten“, den jungen Industrieländern Ostasiens, die in den vergangenen Jahrzehnten einen raschen wirtschaftlichen Aufschwungprozess erlebt haben. Vgl. Peter Berger 1984, S. 17-23; Anton Gälli 1988, S. 61-81 und Hsiao Michael Hsin-hung 1985, S. 149-164.
} 
militärische und wirtschaftliche Unterstützung der USA. Der vorher erstellte sechste Vierjahresplan (1973-1976) ${ }^{485}$ konnte deswegen nicht durchgesetzt werden. In dieser kritischen Lage trat der Staat verstärkt als Investor auf und suchte die Wirtschaft durch einen „Sechsjahresplan“ (1976-1981) ${ }^{486}$ zu unterstützen. Es wurden die sog. „Zehn Nationalen Bauprojekte“(1974-1979) entworfen, von denen sechs der Verbesserung des Verkehrswegenetzes, drei dem Ausbau der Schwerindustrie und eines der Stromversorgung dienten. Daran schlossen sich die sog. „Zwölf Nationalen Projekte“ (1980-1985) als Fortsetzung und Erweiterung an: zur Verbesserung der Bewässerungssysteme, zum Aufbau kultureller Anlagen sowie der Regionalentwicklung. Außerdem förderte der Staat die Entwicklung von kapital- und technologieintensiven Industrien wie Schwerindustrie und Hochtechnologie, Informationstechnologie, Elektronik und Maschinenbau sowie Biotechnologie. ${ }^{487}$ In Hsinchu, in der Nachbarschaft von Forschungsinstituten und den Universitäten Tsing Hua und Chioa Tung, entstand 1979 ein nach dem Modell von Silicon Valley konzipierter Technologiepark. Damit wurde die Ausweiterung privater Klein- und Mittelunternehmen stark unterstützt, vor allem in der Elektronik- und Computerindustrie. Die Etablierung von kleinformatigen Unternehmen und die elastische Arbeitsteilung in Unternehmensnetzwerken erwiesen sich durch ihre flexible Struktur als vorteilhaft. ${ }^{488}$

Das CIECD wurde 1973 zum „Council for Economic Planning and Development“ (CEPD) umgebaut und die „Arbeitsgruppe Arbeitskräfteplanung“ abgeschafft. 1977 wurde der CEPD mit der „Gruppe für Wirtschaft und

\footnotetext{
485 Vgl. Wirtschaftsministerium (Hrsg.) 1973.

486 Vgl. ders. 1976.

487 Vgl. Statistisches Bundesamt Wiesbaden (Hrsg.) 1991, S. 124 f.

488 Vgl. Li Kwoh-ting 1999, S. 274.
} 
Finanzen“ zusammengelegt, die anschließend den „Revidierten Plan zur Ausbildung von Arbeitskräften“ veröffentlichte. Die Ausbildung wurde im sechsjährigen (19761981) und zehnjährigen (1980-1989) Plan zum wirtschaftlichen Aufbau Taiwans berücksichtigt. Der von dem CEPD vorgelegte Plan (1980-1984) war zwar stark volkswirtschaftlich ausgerichtet, jedoch ohne Festlegung auf eine bestimmte prozentuale Verteilung auf einzelne Bildungszweige. ${ }^{489}$ In den 1980er Jahren verlor sich langsam das Regulierungsbestreben in Bezug auf die Arbeitskräfteplanung, vor allem nach Aufhebung des Kriegsrechts 1987, an seine Stelle traten Liberalisierung und Internationalisierung.

\subsubsection{Die „Pläne zur Ausbildung von Arbeitskräften“}

Im „Plan zur Ausbildung von Arbeitskräften“ von 1966 wurde Folgendes festgelegt:

(1) Verlängerung der Schulpflichtzeit von sechs auf neun Jahre;

(2) Neuregulierung der Schulplätze in der Sekundarstufe II

(3) Gestaltung der fünfjährigen Fachschule

(4) Neuregulierung der Fächerstruktur und die Kontrolle über Studentenzahlen in der Tertiärstufe.

\footnotetext{
489 Council for International Economic Cooperation und Development (Hrsg.): „Bericht über die Ausbildung von Arbeitskräften in Taiwan“ (1966), „Revidierter Plan zur Ausbildung von Arbeitskräften (1968), „Dritter Plan zur Ausbildung von Arbeitskräften“ (1970), Vierter Plan zur Ausbildung von Arbeitskräften (1971-1981) (1972), CEPD (Hrsg.) 1986: Mittel- und langfristiger Plan zur Ausbildung von Arbeitskräften zum Wirtschaftsaufbau der Republik China.
} 
Die folgende Abbildung stellt das geplante Bildungssystem vor:

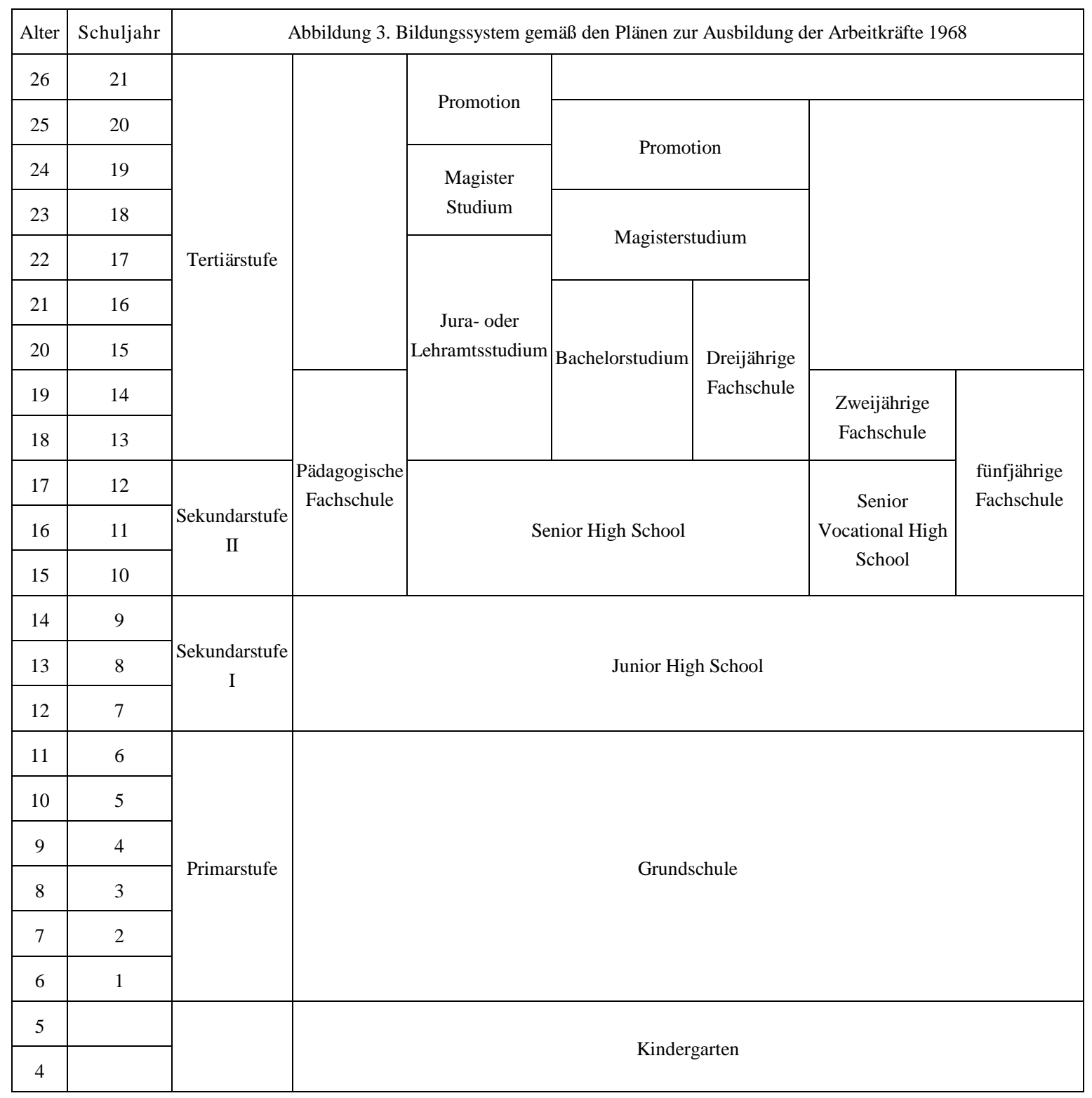

\section{(1) Verlängerung der Schulpflichtzeit von sechs auf neun Jahre}

Bereits 1966 war die Verlängerung der Schulpflichtzeit von der KMT-Regierung zur dringlichsten Aufgabe erklärt worden. Die Gründe waren zum einen die massive Arbeitslosigkeit der Zwölf- bis Vierzehnjährigen und die Linderung von schulischem Stress der Grundschulkinder, ${ }^{490}$ zum anderen der Druck der internationalen Konkurrenz. Im Jahr 1959 hatte die UNESCO eine Tagung in Karachi, Pakistan, für 
ihre Mitgliederländer in Asien zur Förderung der Schulpflicht im Elementarbereich ausgerichtet, in der eine langfristige Planung für die Bildungsentwicklung der nächsten zwanzig Jahre, der sog. „Karachi-Plan“, vorgelegt wurde, wonach die Schulpflicht mindestens sechs Jahre dauern und in allen Unterzeichnerländern bis 1980 realisiert werden sollte. ${ }^{491}$ In Taiwan war die sechsjährige Schulpflicht bereits vor dem Zweiten Weltkrieg eingeführt worden; zu Beginn der 1950er-Jahre lag die relative Schulbesuchsrate bei $90 \%$. Das Vorbild waren indes die Industrieländer obwohl es Taiwan an entsprechenden Finanzmitteln fehlte - mit einer Schulpflichtzeit von neun Jahren, um die internationale Konkurrenzfähigkeit zu stärken. ${ }^{492}$

Nach den gescheiterten Wiedereroberungsplänen flossen deutlich mehr Staatsgelder auf den Sektor des bildungspolitischen, kulturellen, wirtschaftlichen und sozialen Aufbaus, ${ }^{493}$ da dies im Sinne Chiang Kai-sheks und seiner Politik der „Vereinigung Chinas unter den Drei Prinzipien des Volkes“ war und der „geistigen Verteidigung“ und ideologischen Konkurrenzsteigerung $\mathrm{zu}$ den chinesischen Kommunisten diente. Die Einführung der neunjährigen Schulpflicht wurde nun mit der in dieser Zeit ausgerufenen „Bewegung zur Bewahrung und Restauration der chinesischen Kultur“ ${ }^{494}$ verbunden, um die Schülerpersönlichkeit nach der konfuzianischen Morallehre zu bilden. 1967 legte das Erziehungsministerium der Gesetzgebenden Versammlung im Staatsrat die „Richtlinien für eine neunjährige Schulpflicht“ vor, und am 27. Januar 1968 wurde das neue Gesetz „Bestimmungen zur Durchführung der neunjährigen Pflichtschulerziehung“" sowie die Abschaffung der Junior Vocational High School von Chiang Kai-shek bekannt gegeben.

\footnotetext{
491 Vgl. Bildungsministerium (Hrsg.) 1974, S. 324; Kang Tai-kuang und Shen Tseng-chi 1965, S. 3035 .

492 Vgl. Chang Chi-yun 1982 (a), S. 217f. und Li Chuan-ling 2006, S. 91-96.

493 Vgl. CEPD (Hrsg.) 1978, S. 157 und ders. 1995, S. 160.

494 Vgl. 6.2.4. in dieser Arbeit.
} 
Im Jahr 1967 hatte die relative Schulbesuchsrate in der elementaren Bildung immerhin schon 97,52\% erreicht (Tabelle 7a), jedoch lag die Einschulungsrate bei den Junior und Junior Vocational High Schools nur bei 63,66\% (Tabelle 7a). Es fehlte also zur neunjährigen Schulpflicht ein enormer Teil an Plätzen in der Junior High School, von denen 1968 neue aufgebaut wurden, um dem zu erwartenden Schüleransturm gerecht zu werden. Zugleich wurde ein Teil der bisherigen Junior Vocational High Schools in Junior High Schools umgewandelt. Gemäß den Richtlinien von 1964 war jede Klasse dort für 40 bis 50 Schüler vorgesehen und die Anzahl der Klassen in einer Schule auf 36 begrenzt. ${ }^{495}$ Da die neunjährige Schulpflicht in kurzer Zeit umgesetzt wurde, entstanden nun ähnliche Probleme wie in den Grundschulen in Form einer Riesenanzahl an Klassen in einer Schule und enormen Klassengrößen. Anhand der Statistik (Tabelle 11) sind diese Probleme gut zu erkennen. Im Zeitraum von 1968 bis 1978 hat sich die Anzahl der Schüler in den Junior High Schools von 617.225 auf 1.082.074 fast verdoppelt. Im Jahr 1978 erhielten 94,08 \% der Absolventen der Grundschule einen Platz an der Junior High School, obwohl im besagten Zeitraum nur 145 Schulen (von 1968: 487 auf 1978: 632) hinzukamen. Die Anzahl der Schulklassen stieg von 1968 bis 1978 von 11.459 auf 21.799. Die durchschnittliche Anzahl der Klassen pro Schule stieg von 23,53 auf 34,49 und die durchschnittliche Klassenstärke von 53,86 auf 49,64. 1986 erreichte die Eintrittsrate der Grundschulabsolventen in die Junior High School 99,04 \%. Es gab insgesamt 676 Schulen mit 23.352 Klassen und 1.052.993 Schüler. Die durchschnittliche Anzahl der Klassen in einer Schule lag bei 34,54 und die durchschnittliche Klassenstärke lag bei 45,09 Schülern. In den Großstädten lagen die Zahlen oft viel höher als der Durchschnittswert, eine Klasse mit 60 bis 70 Schülern war üblich.

495 Vgl. Tang Chih-min 2002, S. 76-78. 


\section{(2) Neuregulierung der Schulplätze in der Sekundarstufe II}

Mit den steigenden Absolventenzahlen entstand massiver Bedarf an Bildungsangeboten in der Sekundarstufe II, daneben bestand die Problematik der Differenzierung, wie die Ausbildung in unterschiedlichen Fachbereichen gestaltet und die Schüler in den Lehrgängen gruppiert werden konnten, waren wichtige Themen der „Arbeitsgruppe Arbeitskräfteplanung“‘. Die Planer trachteten danach, den größten Teil der Absolventen auf die berufs- und fachschulische Erziehung umzulenken und nur einen kleinen Anteil für den allgemeinbildenden Bildungszweig zuzulassen, einerseits um Facharbeitskräfte auszubilden, andererseits um den Drang auf Studienplätze zu mildern. ${ }^{496}$

\section{a. Festlegung der Schulplätze in den Senior High Schools}

Im Jahr 1968 betrug das Verhältnis der Schülerzahlen in den Senior High Schools zu den berufsbildenden Schulen 5,7 zu 4,3 (Tabelle 12). Per Plan sah nun eine Änderung vor: Innerhalb von zehn Jahren, d.h. bis zum Jahr 1974, sollte das Verhältnis von 6 zu 4 auf 4 zu 6 umgewandelt werden. Nach dem „Vierten Plans zur Ausbildung von Arbeitskräften“ 1973 sowie dem „Fünften Plan“ (1976-80) sollte die Verteilung sogar auf 3 zu 7 innerhalb von zehn Jahren, nämlich bis 1983, umgestellt werden. Dieses Ziel wurde bereits 1977 (Tabelle 12) erreicht, und es blieb fast zwanzig Jahre lang konstant. Die Anzahl der Senior High Schools blieb bei ca. 180 und die Gesamtzahl der Schüler bei etwa 185.000.

\section{b. Neuregulierung der Fächerstruktur in den Senior Vocational High Schools}

Die Ausweitung des Bildungsangebotes und die Verbesserung der Landwirtschaftsund Handelserziehung wurden im „Revidierten Plan zur Ausbildung von

496 Vgl. Bildungsministerium (Hrsg.) 1974, S. $28 \mathrm{f}$. 
Arbeitskräften“ sowie im „Vierten Plan zur Ausbildung von Arbeitskräften“ (1972) besonders berücksichtigt. Die Senior Vocational High Schools für Gewerbe, Fischerei und Meereswesen, Handel, Hauswirtschaft, Medizin und Krankenpflege sollten mehr Plätze durch Schaffung neuer Fachrichtungen und Vermehrung der Klassen zur Verfügung stellen. Außerdem sollten gewerbliche Fächer auch in der Agrarwirtschaftsschule eingerichtet werden. In den gewerblichen Schulen sollten neue Lehrgänge gestaltet werden, zugleich sollte die Zahl der Plätze steigen. Von 1968 bis 1986 wuchs die Anzahl der Senior Vocational High Schools von 134 mit 116.206 Schülern auf 204 mit 437.924 Schülern an (Tabelle 12). Die Schülerströme wurden in die unterschiedlichen Fachrichtungen gelenkt. Die Statistik (Tabelle 13 und 13 a) zeigt, dass der Anteil der Schüler im Fachbereich „Gewerbe“ von 21,23\% (31.877 Schüler) im Jahr 1968 auf 49,27\% (215.746 Schüler) im Jahr 1986 stieg, dagegen fiel der Anteil im Fachbereich „Landwirtschaft“" von 16,27\% auf 4,37\% stark ab.

\section{(3) Gestaltung der fünfjährigen Fachschule}

Da die meisten Schüler der Senior High Schools ein Hochschulstudium anstrebten, entstand infolge der begrenzten Anzahl eine starke Konkurrenz um Studienplätze, in den 1960er- und 1970er-Jahren wurden weniger als zwei Fünftel aufgenommen. Die abgelehnten Schüler verfügten aber über keinerlei für den Arbeitsmarkt verwendbare Kenntnisse, sodass sie nicht in der Lage waren, eine Arbeit aufzunehmen. Sowohl in den Stanfordstudien (1962) als auch den Empfehlungen von Dr. Stromnes (1964) wurde die Einrichtung fünfjähriger Fachschulen als Lösung vorgeschlagen. Die Bildungsform der Fachschulen war als „Ersatzform für das Hochschulstudium“ und zugleich als Ort für die Ausbildung von Facharbeitskräften angelegt, denn sie boten einerseits die Vermittlung von Fachkenntnissen und andererseits ein ähnliches 
Prestige wie die Universität oder Fachhochschule. ${ }^{497} 1965$ wurden daraufhin 25 ausgewählte Senior Vocational High Schools der Bereiche Landwirtschaft, Gewerbe, Hauswirtschaft und Handel zu fünfjährigen Fachschulen umgewandelt. ${ }^{498}$

Zugleich begann man die landwirtschaftlichen High Schools zu umfassenden Berufsschulen umzuwandeln. Das Bildungsministerium bewilligt zudem erstmalig die Errichtung privater Fachschulen, insbesondere solche mit industriellen Fachrichtungen. Diese waren nicht nur für Zugänge aus der Sekundarstufe I gedacht, sondern auch für Hochschulbewerber aus der Sekundarstufe II. Der dreijährige Studiengang war für Absolventen der Senior High Schools und der zweijährige für Absolventen der Senior Vocational High Schools und der fünfjährigen Fachschulen gedacht.

Die „Pläne zur Ausbildung von Arbeitskräften“ wurden bis in die 1980er-Jahre hinein umgesetzt (Tabelle 10 und 15). 1967 gab es 57 Fachschulen (darunter 39 private) und 11 industrielle Fachschulen (darunter 9 private). 1972 erreichte die Zunahme ihren Höhepunkt, es wurden 76 Fachschulen (darunter 57 private) errichtet, die Anzahl der industriellen Fachschulen betrug 31 (darunter 29 private), die der Fachschule für Medizin und Pflege lag sie bei 11, für das Handelswesen bei 10, für die Lehrerausbildung bei 9, für Management bei 3, für Fischerei und Meereswesen, Hauswirtschaft, Agrarwirtschaft und Sport bei 2 und für Journalismus, Kunst, Verwaltungswesen und Fremdsprachen bei $1 .{ }^{499}$ Die Anzahl der Fachschulen blieb

\footnotetext{
${ }^{497} \mathrm{Vgl}$. UNESCO und Zentrale Kommission für Zusammenarbeit zwischen schulischer und außerschulischer Ausbildung unter dem Bildungsministerium (Hrsg.) 1964, S. 68-79.

${ }^{498} 1948$ wurden einige fünfjährige landwirtschaftliche Schulen für Absolventen der Grundschule gemäß dem „Edikt der beruflichen Schule” errichtet. 1965 wurde zunächst diese Form der Schule als Studiengang gestaltet. Vgl. Shih Min-fa 1993, S. 588.

${ }^{499}$ Vgl. Chen Tien-hsi, Yu Huan-mo und Chang Pi-chi 1983, S. $92 f$.
} 
bis 1986 konstant, die Gesamtzahl der Schüler an den Fachschulen jedoch wuchs jährlich enorm. Sie lag im Jahr 1967 bei 63.411 (davon 43.804 in privaten Fachschulen, 40.372 im fünfjährigen und 23.039 im dreijährigen Studiengang). 1972 gab es bereits 138.310 Schüler (davon 95.943 in den privaten Fachschulen; 89.745 im fünfjährigen, 22.561 im dreijährigen und 26.004 im zweijährigen Studiengang). Obwohl der Ausbau von 1972 bis 1986 stagnierte, vermehrte sich die Zahl der Schüler ständig. 1986 gab es 244.482 Schüler (davon 190.946 in den privaten Fachschulen, 150.779 im fünfjährigen, 29.532 im dreijährigen und $58.205 \mathrm{im}$ zweijährigen Studiengang). Der Anteil der Schüler in den fünfjährigen industriellen Fachschulen wuchs von 38,10\% (34.190 Schüler) im Jahr 1972 auf 43,91\% (63.818 Schüler) im Jahr $1984^{500}$

Diese rasche Entwicklung führte nicht nur zu Einbußen in der Bildungsqualität, sondern auch zu einem gewissen Sättigungseffekt hinsichtlich der Aufnahmemöglichkeiten am Arbeitsmarkt. Im „,vierten Plan zur Ausbildung von Arbeitskräften“ (1972) und im „Gesonderten Plan zur Ausbildung von Arbeitskräften“ (1977) forderten die Planer, einerseits die Altlasten aus dem zu schnellen Wachstum des Bildungswesens zu beseitigen und eine Evaluierung des Lehrpersonals, der Bildungsinhalte sowie der schulischen Gebäude und Bildungseinrichtungen durchzuführen und andererseits auf die Angleichung der Bildungs- und Ausbildungsströme an die neue Wirtschaftspolitik hinzuwirken. ${ }^{501}$ Die Bemühungen um eine Sicherung des bereits Erreichten wurden durch die seit Langem sinkenden Geburtenraten sowie durch eine verstärkte Allokation der Finanzmittel im Bildungsbereich erleichtert.

\footnotetext{
500 Vgl. Udo Dörnhaus 1988, S. 206.

501 Vgl. Bildungsministerium (Hrsg.) 1974, S. 698-700.
} 
(4) Neuregulierung der Fächerstruktur und Kontrolle über die Erweiterung der Studienplätze

Durch die Bemühungen des „National Council on Long-range Science Development“ (1959-1967) und des Präsidenten der „Academia Sinica“, Wang Shi-chian, war in den 1960er-Jahren die Grundlage für weitere Entwicklungen in Forschung und Ausbildung sowie die wissenschaftliche und technische Zusammenarbeit zwischen Taiwan und den USA gelegt worden. Die Ausbildung von Forschungs- und Lehrkräften wurde im „Zwölfjahresplan für wissenschaftliche Entwicklung“، von 1969 bis 1981 durch Fachinstitute zur Ausbildung von Doktoren und Magistern an den unterschiedlichen Universitäten zielgerichtet gefördert. Diese Forschungsinstitute boten Arbeitsstellen für Wissenschaftler an und bildeten den wissenschaftlichen Nachwuchs und hoch qualifizierte Arbeitskräfte aus, zudem legten sie die Basis für die weitere Gestaltung der Bachelor-Studiengänge sowie für den Transfer der neuen Wissenschafts- und Technikkenntnisse in unterschiedliche Bereiche. ${ }^{502}$ Dieser Plan wurde in die „Pläne zur Ausbildung von Arbeitskräften“ integriert.

Im „Dritten Plan zur Ausbildung von Arbeitskräften“ (1971-1974) und dem „Zwölfjahresplan für wissenschaftliche Entwicklung“ formulierte die „Arbeitsgruppe Arbeitskräfteplanung“، ihre Reformvorschläge zur Behebung der Mängel bei der Gestaltung von Studiengängen und -plätzen an den Hochschulen im Hinblick auf den Bedarf an hoch qualifizierten Arbeitskräften für den Arbeitsmarkt. Das wesentliche Ziel war, Fächergestaltung und Studentenzahlen nach Bedarf auf dem Sektor Hochtechnologie zu regulieren. Nach der Prognose fehlte es an Arbeitskräften in den Bereichen Naturwissenschaft und Technik, dagegen war der Arbeitsmarkt in den

502 Vgl. Chen Tien-hsi, Yu Huan-mo und Chang Pi-chi 1983, S. 103f. 
Bereichen Geistes- und Sozialwissenschaften übersättigt. ${ }^{503}$

In naturwissenschaftlichen und technischen Fächern gab es relativ wenige Studienplätze, zudem gingen um die 30\% der Absolventen entweder zum weiteren Studium ins Ausland oder - wie die Überseechinesen - in ihr Ursprungsland zurück. ${ }^{504}$ Darüber hinaus verfügten fast $70 \%$ der Lehrkräfte nur über einen Bachelortitel. ${ }^{505}$ Das Verhältnis der Studenten zwischen geistes- und sozialwissenschaftlichen Bereichen gegenüber den naturwissenschaftlichen und angewandten naturwissenschaftlichen Bereichen blieb bis 1972 ungefähr bei $52 \mathrm{zu} 48$ (Tabelle 16a), dieses Verhältnis sollte nun auf 45:55 umgestellt werden. Die Fächer, deren Studium sich als ungünstig für die künftige Beschäftigung erwies, sollten ihre Aufnahmezahlen beschneiden. Zugleich sollten die Studenten der Geistes-, Sozialund Wirtschaftswissenschaften ein Nebenfach wählen, um ihre Chancen auf dem Arbeitsmarkt $\mathrm{zu}$ verbessern. ${ }^{506} \mathrm{Um}$ hoch qualifizierte Techniker und Forschungskräfte für die Hochtechnologie-Industrie auszubilden, sollten Naturwissenschaften, Technologie, Medizin sowie Agrarwissenschaften mithilfe des "The Sino-American Economic and Social Development Funds" 507 erweitert werden.

Die Studienplätze sollten jedes Jahr um 3\% erhöht werden, d.h. jährlich wurden nur ca. 680 neue Studienplätze geschaffen, vor allem in Naturwissenschaften,

${ }^{503}$ Vgl. CIECD (Hrsg.) 1970.

504 Vgl. Chen Tien-hsi, Yu Huan-mo und Chang Pi-chi 1983, S. 99-101.

${ }^{505}$ Vgl. CIECD (Hrsg.) 1966.

${ }^{506}$ Vgl. ders. 1970.

507 US-Entwicklungshilfe zur ROC auf Taiwan brachte am Ende Juni $1965 \mathrm{ab}$, anschließend wurde "The Sino-American Economic and Social Development Fund" zur Verwaltung der USEntwicklungshilfe errichtet. 
Technologie, Medizin und Agrarwissenschaften. ${ }^{508}$ Wegen der zunehmenden Anzahl der Prüfungsteilnehmer und dem Anstieg der Hochschulen (Tabelle 10, 16 und 16a) wurde das Aufnahmeverfahren im Jahr 1972 reformiert, die Fachschulen scherten aus dem gemeinsamen Aufnahmeverfahren aus, und von 83.971 Prüfungskandidaten wurden 22.633 Bewerber an 14 Fachhochschulen und 9 Universitäten aufgenommen (Aufnahmequote 26,95\%), davon 12.193 in den naturwissenschaftlichen und technischen sowie medizinischen und agrarwissenschaftlichen Bereichen $(53,87 \%)$ und 10.440 im geistes- und sozialwissenschaftlichen Bereich (46,12\%).

1978 (Tabelle 10, 16 und 16a) bestanden 17 Fachhochschulen und 9 Universitäten. Es gab 94.850 Prüfungsanmeldungen und 26.847 Studenten $(28,30 \%)$ wurden aufgenommen, davon 15.587 in den naturwissenschaftlichen und technischen $(38,00 \%)$ sowie medizinischen und agrarwissenschaftlichen Bereichen $(32,47 \%)$, das waren insgesamt 58,06\% der neu aufgenommenen Studenten. 11.260 Neuzugänge gab es im geisteswissenschaftlichen (18,95\%) und sozialwissenschaftlichen Bereich (24,53\%), das entsprach 41,94\% der insgesamt neu aufgenommenen Studenten.

Ab 1979 wurden einige Fachhochschulen allmählich zu Universitäten erweitert oder fusionierten mit bereits vorhandenen Universitäten. 1984 (Tabelle 10, 16 und 16a) gab es 6 Fachhochschulen und 16 Universitäten, 98.236 Prüfungsanmeldungen und 31.535 Aufnahmen (32,10\%), davon 17.801 in den naturwissenschaftlichen und technischen sowie medizinischen und agrarwissenschaftlichen Bereichen $(42,48 \%)-$ insgesamt $56,45 \%$ der neu aufgenommen Studenten - und 13.734 in geisteswissenschaftlichen und sozialwissenschaftlichen Bereichen $(24,46 \%)$, das entsprach 43,55\% der neu aufgenommenen Studenten. Anhand dieser Angaben zeigt 508 Vgl. Chiu Ai-ling 1998, S.142. 
sich, dass die Aufnahmequote in naturwissenschaftlichen und technischen sowie medizinischen und agrarwissenschaftlichen Bereichen viel höher als in geistes- und sozialwissenschaftlichen Bereichen lag.

\subsubsection{Erweiterung der Lehrerausbildung}

Lehramtsstudenten bekamen einerseits eine staatliche Besoldung, andererseits mussten sie als Gegenleistung auf einer zugewiesenen Stelle einen Pflichtdienst leisten. Aufgrund der raschen und massiven Ausweitung des Bildungsangebotes benötigte man deutlich mehr Lehrkräfte, doch 1950 gab es nur 8 dreijährige Pädagogische Fachschulen für die Ausbildung von Grundschullehrern mit 5651 Teilnehmern (701 Absolventen der Grundschule und 4950 Absolventen der Junior High Schools). 1963 und 1967 wurden neun vorhandene dreijährige Pädagogische Fachschulen zu fünfjährigen Pädagogischen Fachschulen ausgebaut. Die beiden dreijährigen Pädagogischen Fachschulen wurden zu vierjährigen Fachhochschulen für die Ausbildung von Lehrkräften in der High School umorganisiert. ${ }^{509}$ Außerdem übernahmen die „National Taiwan Normal University“ und ein pädagogisches Seminar an der Cheng Chi Universität die Ausbildung von Lehrkräften für Junior und Senior High Schools. Zudem wurde der Zugang zum Lehrerberuf durch die Einrichtung verschiedener Weiterbildungslehrgänge, die am Abend und in den Ferien an allen Pädagogischen Fachhochschulen und Universitäten stattfanden, ermöglicht, weil der Staat keinen großen Aufwand für die Ausbildung der Lehrkräfte treiben

\footnotetext{
${ }^{509}$ Im Jahre 1967, ein Jahr vor Einführung der neunjährigen Schulpflicht, wurde eine im Jahre 1954 neu gegründete Pädagogische Fachschule in Kaohsiung, eigentlich für die Ausbildung von Grundschullehrern zuständig, zur Pädagogischen Fachhochschule erhoben. Eine weitere wurde im Jahre 1971 in Chang Hua gegründet. Die beiden Fachhochschulen und die staatliche Pädagogische Universität zu Taiwan dienten der Ausbildung von Lehrkräften an Junior bzw. Senior High Schools. Beide Hochschulen erhob man in den 80er-Jahren zu Pädagogischen Universitäten.
} 
konnte. Zwischen 1973 und 1977 kam noch ein Fernstudium per Fernsehen hinzu. ${ }^{510}$

\subsubsection{Ungleiche staatliche Unterstützung der allgemeinbildenden und beruflichen Bildungszweige}

Da der Großteil der Staatsausgaben für die Erweiterung der Grundschulen und Junior High Schools verwendet wurde, waren die beruflichen Schulen beim Ausbau auf Subventionen privater Träger angewiesen. Die Gesamtzahl der Senior Vocational High Schools lag 1968 bei 134 (davon 55 private) und wurde bis 1986 auf 204 (davon 115 private) erhöht (Tabelle 14 und 15a). Die Gesamtzahl der Schüler stieg um 321.718 an (von 1968: 116.206 auf 1986: 437.924, um fast das 2,8-Fache), 43,74\% wurden 1968 von privaten Schulen aufgenommen und 61,2\% im Jahr 1986. Die Anzahl der Fachschulen mit fünfjährigem Studiengang (Tabelle 15) stieg von 1968 bis 1986 um 14 Schulen an, davon wurden 13 Fachschulen von privaten Trägern errichtet. Die privaten Fachschulen nahmen fast $75 \%$ der gesamten Schüler und Studenten in den 1970er- und 1980er-Jahren auf (1968: 68,25\%; 1978: 73,33\%; 1986 : 73,68\%). Die Anzahl der Universitäten blieb mit 8 (darunter 2 private) von 1968 bis 1978 fast konstant und die Anzahl der Fachhochschulen nahm von 14 (darunter 10 private) auf 17 (darunter 10 private) zu, was keine entscheidende Änderung darstellt. Die privaten Hochschulen gewannen jedoch an Studenten. Deren Anteil stieg von $50 \%$ in den 1970er Jahren auf gut 60\% in den 1980er Jahren (Tabelle 15a). 1979 wurde der Umbau der Fachhochschulen zu Universitäten eingeleitet und 1985 die Genehmigung für die Errichtung von neuen Fachschulen und Fachhochschulen erneuert. ${ }^{511}$ Nach Aufhebung des Kriegsrechts 1987entstand eine neue Welle der Erweiterung von Hochschulen.

\footnotetext{
510 Vgl. Hsiao Chih-bang 2006, S. 101-122.

511 Ab 1972 wurde die Errichtung der neuen Fachschulen und Fachhochschulen eingeschränkt. Vgl. Bildungsministerium (Hrsg.) 1984, S. 879.
} 
Die privaten Bildungssubventionen trugen zwar dazu bei, den großen Bedarf an Studien- und Ausbildungsplätzen in kurzer Zeit zu decken und die Bildungsausgaben des Staates zu entlasten, es muss jedoch bedacht werden, dass die Bildungsqualität recht unterschiedlich ausfiel. Die meisten privaten Schulen mussten ohne ausreichende finanzielle Unterstützung durch Betriebe und geringe Subventionen der öffentlichen Hand für ihr Überleben kämpfen, folglich verlangten sie hohe Schulgebühren und nahmen so viele Schüler bzw. Studenten wie möglich auf. Diese mussten durchschnittlich mehr als das Dreifache an Gebühren zahlen, bekamen aber häufig eine schlechtere Bildung als diejenigen an einer gleichartigen öffentlichen Schule. Daher brachte der Besuch einer öffentlichen Schulform oft eine höhere soziale Anerkennung mit sich. 


\section{Das einheitliche Aufnahmeprüfungssystem als Methode für Verteilung der Bildungsplätze}

Die Anforderung auf die Chancegleichheit der Bildung wurde innerhalb der Bildungskonzepte des modern-staatlichen Bildungssystem einbezogen, und auch in der Zielsetzung des Bildungssystem (1928) und der Verfassung der Republik China (1947) angenommen. Obwohl alle Bürger das gleiche Recht auf die Bildung haben, in der Realität sind die Bildungsangebote ungleich bzw. in unterschiedlichen Bildungsgängen mit unterschiedlichen Bildungsinhalten, Leistungsanforderungen usw. aufgrund der unterschiedlichen Begabungen, Fertigkeiten, Neigungen und soziale und kulturelle Lage von Schülern, außerdem auch der organisatorischen Notwendigkeit. Selektion- bzw. Verteilungsmaßnahmen dienen dazu, die ungleiche Bildungsangebote und Zugangs-, Bewertungs- und Selektionskriterien im Schulsystem zu bestimmen. Die Erstellung der Selektion- bzw. Verteilungsmaßnahmen sind häufig eng mit den kulturellen, sozial-ökonomischen und politischen Gegebenheiten verankert. In Taiwan wurde die Zuteilung der Bildungschancen in der Phase der Schulpflicht vom Wohnort bestimmt und nach der Schulpflicht wurde mit Hilfe der gemeinschaftlichen Verteilungsverfahren durch einheitliche Aufnahmeprüfung gemäß dem Leistungsprinzip durchgeführt. Dies System war eine Modifikation von Nankinger KMT-Regierung aus der chinesischen Tradition.

8.1. Einführung der Abschlussprüfung und des einheitlichen Aufnahmeprüfungssystems zur Hochschule auf dem chinesischen Festland in den 1930er Jahren

Die Abschaffung des jahrtausendealten kaiserlichen Beamtenprüfungssystem 1905 führte zu schweren Erschütterungen in der chinesischen Gesellschaft und zur Orientierungslosigkeit der Gelehrten. Auch in der „Bewegung des Vierten Mai 
1919“ wurden Prüfungen in der Schule kritisch gesehen und die Abschaffung der Leistungskontrolle durchgesetzt. Mit dem Zusammenbruch des Prüfungswesens nach Gründung der Republik geriet China aber in ein „Unterrichtschaos“. Zugleich gab es kein einheitliches Prüfungssystem vor Eintritt in die höhere Bildung. Die Aufnahme der neuen Schüler und Studenten war Sache der einzelnen Schule bzw. Universitäten. Anforderungen und Aufnahmeverfahren waren deswegen von Schule zu Schule bzw. Universität $\mathrm{zu}$ Universität sehr unterschiedlich, sodass sich die Bewerber für unterschiedliche Aufnahmeprüfungen vorbereiten mussten. Außerdem konnten die Studienplätze mit Geld erworben werden, ${ }^{512}$ was jedoch der Vorstellung von sozialer Gerechtigkeit entgegen stand, da Aufstiegsmöglichkeiten nach der individuellen Leistung geregelt werden sollten.

Die Forderung nach Einführung eines Prüfungssystems im staatlichen Bildungswesen in den 1920er- und 30er-Jahren hing zunächst mit der Vereinheitlichung der Lehrinhalte und der Einführung der Parteierziehung zusammen. Als die KMTRegierung 1928 an die Macht kam, versuchte sie das liberale und dezentrale Bildungswesen laut Schuledikt aus dem Jahr 1922 zu vereinheitlichen. Der orthodoxe Politiker Tai Chi-tao, ${ }^{513}$ von 1928 bis 1948 als Präsident des Staatsrates für die Prüfung und Auswahl der Beamten zuständig, wies 1931 darauf hin, dass die dauernden Unruhen unter den Studenten und das Absinken der erzieherischen Qualität mit der Abschaffung des Prüfungssystems zu tun hätten. ${ }^{514}$ Nach seiner Ansicht sollten Gedanken und politische Identität der Jugendlichen durch vom Staat

\footnotetext{
${ }^{512}$ Vgl. Kuan Mei-jung 2008 S.97-105 und Yang Hsueh-wei (Hrsg.) 1992, S. 574.

513 Tai Chi-tao (1891-1949) war etwa 14 Jahre lang der persönliche Sekretär von Sun Yat-sen und nach dessen Tod 1925 der stärkste Vertreter einer antikommunistischen Interpretation der „Drei Prinzipien des Volkes“. Er versuchte die Unterschiede zwischen KMT und KPCh als einer Partei gegen die Tradition aufzuzeigen. Vgl. Tu Chian-feng 1989.

${ }^{514}$ Vgl. Lu Fang-sang 1994, S. 61-64.
} 
bestimmte Lehrinhalte zusammengehalten werden. Ein Prüfungssystem motiviere bzw. zwinge die Schüler zum Studium der Lehrstoffe, so wie im alten China mithilfe des kaiserlichen Beamtenprüfungssystems die erwünschten Werte und Normen unter den Gelehrten und in der Gesellschaft vereinheitlicht und verbreitet wurden. ${ }^{515} \mathrm{Um}$ die Qualität der schulischen Erziehung zu erhöhen, schlug die Bildungskommission des Völkerbunds 1932 vor, eine vom Staat gestaltete Aufnahmeprüfung zur Hochschule einzuführen und eine zentrale Aufnahmeprüfungskommission einzusetzen, die die Kriterien für die Qualifikation der Prüfungsteilnehmer und Aufnahmebarrieren bestimmen sollte. Den Prüfungsteilnehmern sollten aufgrund ihrer Ergebnisse ein Studienplatz zugewiesen werden. ${ }^{516}$

Zunächst wurde ein Abschlussprüfungssystem für Schüler der Mittelschule eingeführt, das an das Abitur nach europäischem Muster ${ }^{517}$ angeglichen worden war. Ab 1929 wurden die Lehrinhalte den „Richtlinien für schulische Lehrbücher“ angepasst. Gemäß der „Ordnung für den Abschluss in der Mittelschule“ von 1932 legten die Absolventen eine einheitliche staatliche Abschlussprüfung ab. Die Prüfungsfächer waren Staatsbürgerkunde (Lehrinhalt war Parteiideologie der KMT), Chinesisch, Mathematik, Physik und Chemie, Biologie, Geschichte und Geographie für die Junior High School; für die Senior High School kam noch eine Fremdsprache hinzu. Die Abschlussergebnisse bestanden $\mathrm{zu}$ 60\% aus der Prüfung und $\mathrm{zu} 40 \%$ aus der dreijährigen Schulleistung. Bestanden die Schüler mehr als drei Fächer nicht, mussten sie die Prüfung wiederholen. Außerdem wurden die gesamten Leistungen individuell

\footnotetext{
515 Vgl. Tai Chi-tao 1984, S. 310f.

516 Vgl. Die Bildungskommission des Völkerbunds 1963, S.204; Lin Chen-chen und Lin Hung-chun 2002, S. 835-862.

517 In Europa, z. B. in Preußen, gab es seit 1788 die Abiturprüfung als Zertifikat für die Bewerbung um einen Studienplatz und eine Amts- oder Arbeitsstelle. Vgl. Max A. Eckstein und Harold J. Noah 1996, S. 8-10.
} 
und schulbezogen verglichen und in einer Rangliste veröffentlicht. ${ }^{518}$

Während des Kriegs, von 1938 bis 1940, wurde ein einheitliches Aufnahmeprüfungssystem zur Hochschule unter dem damaligem Bildungsminister Chen Li-fu eingeführt. ${ }^{519}$ Den Hochschulen wurden unterschiedliche Regionen des Landes zugeteilt und die Prüfungen mit einheitlichen Aufgaben, an einem Prüfungstermin und gemeinsamen Verteilungsverfahren durchgeführt. ${ }^{520}$ Damit waren die Prüfungen wieder unter Aufsicht des staatlichen Erziehungs- und Bildungswesens und hatten die gleiche Funktion wie das kaiserliche Beamtenprüfungssystem im alten China: Sie dienten der Auswahl begabter Schüler und der Kontrolle über schulische Leistung und Qualität der Schule sowie der Vereinheitlichung und Verbreitung der Staatsideologie, zugleich aber auch der sozialen Gerechtigkeit durch die Zuteilung der Bildungsplätze nach der individuellen Leistung.

\subsection{Einführung eines einheitlichen Aufnahmeprüfungssystems in Taiwan}

Die Aufnahme der neuen Schüler gehörte vor der Ankunft Chiang Kai-sheks auch in Taiwan zu den Obliegenheiten der einzelnen Schulen, jede Schule führte selbstständig ein Aufnahmeverfahren durch, nachdem sie die Schüler bei der Schule, die sie besuchen wollten, angemeldet hatten. Viele Schüler strebten nach der Rückgabe Taiwans an China nach höherer Bildung, und nach 1949 strömten zudem immer mehr Schüler vom chinesischen Festland ins Land, was die Konkurrenz um die Bildungsplätze weiter verschärfte. ${ }^{521}$ Zudem wurde im Zuge des „Aufstands am 28.

\footnotetext{
518 Vgl. Bildungsministerium (Hrsg.) 1957, S. 220.

519 Vgl. Cheng Teng-yun 1994, S. 496.

520 Vgl. Chen Li-fu 1973, S. 58f.

521 Vgl. 7.3.2. in dieser Arbeit.
} 
Februar $1947^{\text {‘522 }}$ nachdrücklich Offenheit und Gerechtigkeit bei der Zuteilung der Plätze zur weiterführenden Bildung gefordert.

Die Durchführung der Aufnahmeprüfung war sehr komplex: von der Anmeldung der Prüfungsteilnehmer, der Erstellung der Prüfungsaufgaben (zur selben Zeit mussten die Aufgaben der Aufnahmeprüfung zur Mittelschule in Staatsbürgerkunde, Chinesisch, Geschichte und Geographie, Mathematik, Englisch, Physik, Chemie und Naturkunde erstellt werden), Durchführung der Prüfung, Korrektur der bearbeiteten Aufgaben, Eintrag der Ergebnisse der Prüfungsteilnehmer bis zur Veröffentlichung der aufgenommenen Prüflinge. Dadurch war die einzelnen Schulen überfordert, vor allem bei den rasch anwachsenden Schülerzahlen nach 1950. 1952 wurde zunächst in fünf Mittelschulen der Hauptstadt Taipei ein ,gemeinschaftliches Aufnahmeverfahren gleicher Schulformen mit einheitlichen Prüfungsaufgaben“ probeweise eingeführt. Die Obliegenheiten wurden von den Schulen gemeinsam getragen, Bewerbung und Prüfung galten für alle fünf Mittelschulen. 1958 nahmen immer mehr Mittelschulen in Taipei daran teil, später wurde das Modell auch in anderen Landkreisen angewandt. ${ }^{523}$ 1952 wurde die neue Richtlinie für Lehrinhalte in den Fächern Staatsbürgerkunde, Chinesisch, Geschichte und Geographie zum Zweck der Verstärkung der „Bildung des Volksgeistes“ in der Mittelschule erlassen. Nachfolgend wurden zwei Maßnahmen wieder eingesetzt, die die KMT-Regierung in den 1930er- und 1940er-Jahren verwendet hatte, nämlich die Abschlussprüfung in den Senior High Schools und die Aufnahmeprüfung zur Hochschule. Der Zweck war Bildungsminister Chang Chi-yun zufolge, einerseits alle Schulen und Hochschulen des Landes zur „Bildung des Volksgeistes“ zu bewegen, andererseits gewann das Bildungs-

\footnotetext{
$522 \mathrm{Vgl}$. 6.2.4.1. in dieser Arbeit.

523 Vgl. Lin Chuan-i 2002, S. 40.
} 
ministerium dadurch nahezu vollständige Daten über die schulische Realität, die wiederum als Basis zur künftigen Verbesserung von Mittelschule und Hochschule fungieren konnten. ${ }^{524}$ Wegen der Ähnlichkeit zur Beamtenprüfung wurden im nächsten Jahr die Abschlussprüfung in den Senior High Schools abgeschafft, stattdessen wurde wieder das Aufnahmeverfahren mit einheitlicher Aufnahmeprüfung zur Hochschule praktiziert. ${ }^{525}$

In der Folge war die Zuteilung der Schule für die Pflichtschulzeit vom Wohnort abhängig und ein Platz im weiterführenden Schulsystem von den Ergebnissen der Aufnahmeprüfung. Um Fehler zu vermeiden, wurden die Prüfungsaufgaben von mindestens zwei Personen und seit den 1980er-Jahren per Computer korrigiert. Die Prüfungsergebnisse wurden veröffentlicht, es sollte bei der Verteilung der Studienplätze so offen wie möglich zugehen, um Einflüsse und Einmischungen durch Vorurteile, Privilegien oder gar Bestechung vom Verteilungsprozess fernzuhalten. Eine objektiv fundierte und gerechte Aufstiegschance sollte für jeden Prüfungsteilnehmer gewährleistet sein. Ob ein Bildungsbewerber für die Senior High School, Senior Vocational High School, Fachschule, Fachhochschule oder Universität zugelassen wurde, hing ausschließlich vom Ergebnis der Prüfung und nicht von der schulischen Leistung, der Herkunft oder sonstigen Gründen ab. Die Schüler mussten selbstständig die Leiter heraufklettern, um einen Ausbildungsplatz bzw. künftige Aufstiegsmöglichkeiten zu erhalten. Das hieß, sobald die Schüler in die Junior High School eintraten, verbrachten sie viele Stunden mit der Vorbereitung auf die Aufnahmeprüfung zur Sekundarstufe II. Anschließend kämpften sie um die Plätze in der Hochschule.

\footnotetext{
524 Vgl. Chang Chi-yun 1956, S. 35.

525 Vgl. Liu Hsien-yun 1956, S. 13.
} 


\subsection{Die Aufnahmeprüfung zur Hochschule}

Die Entwicklung des Aufnahmeprüfungssystems zur Hochschule kann man in drei Phasen einteilen: Aufbauphase (1954-1971), Erneuerungsphase (1972-1986) und Liberalisierungsphase (1987 bis in die Gegenwart). Im Folgenden werden die Besonderheiten der Aufbauphase und der Erneuerungsphase, also des Verfahrens vor Aufhebung des Kriegsrechts, genauer vorgestellt.

\subsubsection{Die Aufbauphase (1954-1971)}

Das Aufnahmeprüfungssystem zur Hochschule wurde ab 1954 allmählich in Taiwan aufgebaut. Zunächt wurde eine zuständige Organisation errichtet, anschließend wurden die entsprechenden Regel und Durchführungsmaßnamen für die gemeinschaftliche Aufnahmeprüfung zur Hochschule erstellt.

\subsubsection{Die Organisation des Aufnahmeprüfungssystems}

1954 wurde in vier unterschiedlichen Hochschulen, der National Taiwan University, der Pädagogischen Fachhochschule in Taipei, der Landwirtschaftlichen Fachhochschule in Taichung und der Technischen Fachhochschule in Tainan, ein Aufnahmeverfahren mit einheitlicher Aufnahmeprüfung eingeführt. 1956 und 1957 führten 6 Universitäten, 5 Fachhochschulen, 6 Fachschulen und 11 Militärschulen das Aufnahmeverfahren gemeinschaftlich durch. ${ }^{526}$ Obgleich die Hochschulen unterschiedliche Funktionen hatten, griffen überall die Maßnahmen zur Durchsetzung der „Erziehung mit Feder und Waffe“ Chiang Kai-sheks. Da die Militärschulen die letzte Wahl der Prüfungsteilnehmer darstellte, bekamen diese keine ausreichenden Bewerberzahlen und traten ab 1958 aus dem gemeinschaftlichen Verfahren aus. ${ }^{527}$

\footnotetext{
526 Vgl. Bildungsministerium (Hrsg.) 1974, S. 729.

527 Vgl. Kuang Mei-jung 2004, S. 69.
} 
Zur Durchführung des Aufnahmeverfahrens wurde 1957 die „Kommission zur Maßnahmenerstellung und Durchführung der gemeinschaftlichen Aufnahmeverfahren für neue Studenten zur Hochschule“ errichtet, eine Ad-hoc-Kommission ohne langfristig zuständige Personen. Jedes Jahr trafen die Präsidenten der Universitäten und Fachhochschulen zusammen, um eine Universität auszuwählen, die alle Aufgaben zu übernehmen hatte: die Anmeldung, Erstellung und Korrektur der Prüfungsaufgaben, Berechnung der Ergebnisse sowie die Verteilung der Schüler und Studenten auf die Hochschulen. Die Mitglieder der Kommission verfügten jedoch über keine Entscheidungsmacht, sie stand allein Chiang Kai-shek zu, also auch die Entscheidung über die Aufnahme der neuen Schüler bzw. Studenten. Nach der Durchführung gingen alle zuständigen Personen wieder an ihre bisherige Stelle zurück. $^{528}$

\subsubsection{Voraussetzungen für die Teilnahme an der Aufnahmeprüfung}

Gemäß Hochschulgesetz $\S 26$ waren die Voraussetzungen zum Eintritt in die Hochschule folgendermaßen bestimmt: Der Bewerber sollte eine öffentliche oder private Senior High School oder eine entsprechende Stufe der Schule absolviert und die Aufnahmeprüfung bestanden haben. Die Absolventen der Senior Vocational High School und der fünfjährigen Fachschule durften zwar an der Aufnahmeprüfung teilnehmen, aber sie durften sich nur um eine Ausbildung in adäquaten Fächern bewerben, die ihrem Abschluss in den Senior Vocational High Schools und fünfjährigen Fachschulen entsprachen. ${ }^{529}$ Ab 1969 durften auch die Absolventen der Militärschulen teilnehmen. Ab 1973 wurde die Bewerbungsbeschränkungen für die Absolventen der Senior Vocational High Schools und der fünfjährigen Fachschulen

\footnotetext{
${ }^{528}$ Vgl. Bildungsministerium (Hrsg.) 1974, S. $730 f$.

529 Vgl. ebd., S. 732.
} 
aufgehoben, sodass sie nunmehr alle Hochschulfächer studieren konnten. ${ }^{530}$

\subsubsection{Gliederung der Studienfächer und Gruppierung der Prüfungsteilnehmer}

Die Fächer an den Universitäten, Fachhochschulen und Fachschulen waren sehr vielseitig gestaltet, und jedes Fach hatte besondere Anforderungen an die Kenntnisse und Begabungen der Studenten. Außerdem gab es immer mehr Prüfungsteilnehmer mit unterschiedlichen Fachkompetenzen und Begabungen, deshalb fasste das Bildungsministerium ähnliche Fächer zu einer Gruppe zusammen, in der die Prüfungsteilnehmer untereinander konkurrierten. Sie wurden zunächst in acht Fakultäten gegliedert - Naturwissenschaften, Technik, Medizin, Agrarwissenschaften, Geisteswissenschaften, Pädagogik, Jura sowie Sozial- und Wirtschaftswissenschaften - und von 1954 bis 1965 wiederum in drei Gruppen eingeteilt:

Gruppe A beinhaltete Fächer in den Fakultäten Naturwissenschaften, Technik, Medizin sowie die Fächer Agrartechnik und Agrarchemie in der Fakultät für Agrarwissenschaften, ferner das Fach Gewerbliche Erziehung in der Pädagogischen Fakultät sowie die Fächer Verwaltungswesen für Verkehr und Industriemanagement der Fakultät für Sozial- und Wirtschaftswissenschaften.

Gruppe B beinhaltete Fächer der Fakultäten Geisteswissenschaften, Pädagogik, Jura sowie Sozial- und Wirtschaftswissenschaften.

Gruppe C umfasste Fächer der Fakultät für Agrarwissenschaften sowie für Naturwissenschaften wie Zoologie, Pflanzenlehre, Biologie, Naturgeschichte, ferner Fächer wie Sportwissenschaft, Hygieneerziehung und Hauswirtschaft. ${ }^{531}$

\footnotetext{
${ }^{530}$ Vgl. ebd.

531 Vgl. Bildungsministerium (Hrsg.) 1974, S. 733.
} 
1966 wurde die Gruppierung erneuert. Obwohl geistes- und sozialwissenschaftliche Fächer in einer Gruppe zusammengefasst worden waren, stellten sie ganz unterschiedliche Forderungen an die Fachkompetenz der Studenten. ${ }^{532}$ Zudem gab es zunehmend mehr Prüfungsanmeldungen in der Gruppe A, daher sollte diese weiter differenziert werden, nicht zuletzt um die Arbeitslast beim Aufnahmeverfahren zu mildern. Daher wurde die Fakultät Medizin zur eigenständigen Gruppe C und Jura sowie Sozial- und Wirtschaftswissenschaften zur Gruppe D zusammengefasst. ${ }^{533}$

\subsubsection{Die Prüfungsfächer}

Die Prüfungsfächer wurden in zwei Formen unterteilt, in gemeinsame und Gruppenfächer. Die Aufgaben der gemeinsamen Fächer mussten von allen Prüfungsteilnehmern gelöst werden. Daneben hatte jede Gruppe ihre eigenen Prüfungsfächer. Alle Schüler waren gezwungen, die vom Staat herausgegebenen Lehrbücher für die gemeinsamen Prüfungen zu studieren, von denen es drei gab:

(1),,Drei Prinzipien des Volkes“: ab 1953 Prüfungsfach in der staatlichen Prüfung. ${ }^{534}$ Es wurden Erläuterungen der Thesen von Sun Yat-sen abgefragt oder die Verbindung zu aktuellen politischen Themen hergestellt.

(2)Chinesisch: Nach Meinung Chiangs trug Chinesisch zur „Bildung des Volksgeistes" bei, denn damit würden die Jugendlichen in der chinesischen Kultur gebildet und ihr nationales Bewusstsein sowie die Ablehnung des Kommunismus gestärkt. Das Schreiben von Aufsätzen galt wie in der kaiserlichen Beamtenprüfung als einer der wichtigsten Teile. Oft wurden politische Ereignisse als Themen gewählt. Die Prüfungsteilnehmer sollten mit der politischen Ideologie und der konfuzianischen

\footnotetext{
532 Vgl. ebd.

533 Vgl. Chiu Ai-ling 1998, S. 65.

534 Vgl. Bildungsministerium (Hrsg.) 1957, S. 502.
} 
Morallehre übereinstimmende Argumente nennen. ${ }^{535}$

(3)Englisch: Englisch war dominierende internationale Sprache, außerdem stand Taiwan durch die US-Entwicklungshilfe in starker Abhängigkeit zu den USA. ${ }^{536}$ Der Erwerb der englischen Sprache wurde daher als wichtige Voraussetzung für das Studium an der Hochschule mit einbezogen.

Die Entscheidung für das jeweilige Gruppenfach war oft schon durch das Kurssystem der Senior High School vorbestimmt. Das hieß, dass Schüler, die sich für die Gruppe A vorbereiteten, sich nur noch wenig um die Fächer Geschichte und Geographie kümmerten, während die Schüler der Gruppe B kaum noch Physik, Chemie und Biologie lernten. Die Beamten versuchten daher, diesbezüglich die Schulerziehung zu ändern. In der Frage, ob die Erziehung der Senior High School vorderhand als Allgemeinbildung, zur Verstärkung der „Bildung des Volksgeistes“ oder zur Vorbereitung auf die Universität behandelt werden sollte, schwankten sie mit ihrer Meinung, was sich bei der Gestaltung der Prüfungsfächer für die unterschiedlichen Gruppen widerspiegelte (Tabelle 17). 1954 wurden als gemeinsame Prüfungsfächer Chinesisch, Englisch und „Drei Prinzipien des Volkes“ genannt, 1956 kamen Chinesische Geschichte und Geographie hinzu.

Im Jahr 1958 wurde die Gruppierung abgeschafft und alle Prüfungsteilnehmer in allen Fächern geprüft. Die aufgenommenen Studenten wurden nach Fakultäten eingeteilt. Doch diese Maßnahmen wurden im nächsten Jahr wieder aufgehoben. Im Zeitraum von 1966 bis 1983 gab es feste Gruppenfächer: Physik, Chemie und Mathematik für die naturwissenschaftliche Gruppe, Chinesische und Weltgeschichte, Chinesische und

\footnotetext{
535 Vgl. Kuang Mei-jung 2004, S. 154-162.

536 Vgl. Chiu Ai-ling 1998, S. 65 f.
} 
Weltgeographie sowie Mathematik für die sozialwissenschaftliche Gruppe, Chemie, Biologie und Mathematik für die naturwissenschaftliche Gruppe sowie Chinesische und Weltgeschichte, Chinesische und Weltgeographie und Mathematik für die sozialwissenschaftliche Gruppe. ${ }^{537}$

\begin{tabular}{|c|c|c|}
\hline Jahr & Gemeinsame Prüfungsfächer & Gruppenfächer \\
\hline 1954 & $\begin{array}{l}\text { „Drei Prinzipien des Volkes“ } \\
\text { Chinesisch } \\
\text { Englisch }\end{array}$ & $\begin{array}{l}\text { Gruppe A - Physik, Chemie, Mathematik für die } \\
\text { naturwissenschaftliche Gruppe } \\
\text { Gruppe B - Chinesische- und Weltgeschichte, Chinesische und } \\
\text { Weltgeographie, Mathematik für die Sozialwissenschaftliche } \\
\text { Gruppe } \\
\text { Gruppe C - Chemie, Biologie, Mathematik für die } \\
\text { sozialwissenschaftliche Gruppe }\end{array}$ \\
\hline $1956-57$ & $\begin{array}{l}\text { „Drei Prinzipien des Volkes“ } \\
\text { Chinesisch } \\
\text { Englisch } \\
\text { chinesische Geschichte und Geographie }\end{array}$ & $\begin{array}{l}\text { Gruppe A - Physik, Chemie, Mathematik für die } \\
\text { naturwissenschaftliche Gruppe } \\
\text { Gruppe B - Weltgeschichte, Weltgeographie, Mathematik für } \\
\text { die sozialwissenschaftliche Gruppe } \\
\text { Gruppe C - Chemie, Biologie, Mathematik für die } \\
\text { sozialwissenschaftliche Gruppe }\end{array}$ \\
\hline 1958 & \multicolumn{2}{|c|}{$\begin{array}{l}\text { „Drei Prinzipien des Volkes“, Chinesisch, Englisch, } \\
\text { Geschichte und Geographie in China sowie Physik und Chemie }\end{array}$} \\
\hline $1959-1965$ & \multicolumn{2}{|l|}{ Wie 1954} \\
\hline $\begin{array}{l}1966 \\
-1983\end{array}$ & $\begin{array}{l}\text { „Drei Prinzipien des Volkes“ } \\
\text { Chinesisch } \\
\text { Englisch }\end{array}$ & $\begin{array}{l}\text { Gruppe A - Physik, Chemie, Mathematik für die } \\
\text { naturwissenschaftliche Gruppe } \\
\text { Gruppe B - Chinesische- und Weltgeschichte, Chinesische- } \\
\text { und Weltgeographie, Mathematik für die } \\
\text { sozialwissenschaftliche Gruppe } \\
\text { Gruppe C - Chemie, Biologie, Mathematik für die } \\
\text { naturwissenschaftliche Gruppe } \\
\text { Gruppe D - Chinesische- und Weltgeschichte, Chinesische- } \\
\text { und Weltgeographie, Mathematik für die } \\
\text { sozialwissenschaftliche Gruppe }\end{array}$ \\
\hline
\end{tabular}

Quelle: Bildungsministerium 1974 S. $733 f$.

537 Obwohl die Gruppenfächer von Gruppe B (Geisteswissenschaften) und D (Jura, Sozial- und Wirtschaftswissenschaften Fächer) identisch waren, wurden die Prüfungsergebnisse mit unterschiedlichem Gewicht berechnet. 


\subsubsection{Prüfungsaufgaben und -verfahren}

Um Gerechtigkeit, Offenheit und Objektivität zu gewährleisten, wurden die Prüfungen unter strengen Vorsichtsmaßnahmen durchgeführt. Jedes Jahr wählten die Mitglieder der „Kommission für die gemeinschaftlichen Aufnahmeverfahren zur Hochschule“ drei bis vier Hochschulen aus, deren Professoren dann für die Erstellung der Prüfungsaufgaben zuständig waren. Voraussetzung war, dass keines ihrer Kinder oder andere Verwandte an der Aufnahmeprüfung teilnahmen und sie keine Beziehung zu privaten Schulen bzw. Nachhilfeinstituten hatten.

Die Prüfungsaufgaben hatten sich an den Lehrinhalten der Senior High School zu orientieren. Sie fragten standardisierte Lösungen bzw. Argumente ab, die in den einheitlichen Lehrbüchern vorgegeben waren. Für jedes Prüfungsfach wurden zwei bis vier Variationen mit unterschiedlichen Schwierigkeitsstufen erstellt. Kurz vor der Prüfung wurde eine davon ausgewählt. Es gab die Formen Argumente, Fragen und Antworten, Erläuterungen, Lückentexte, Rechnen, Aufsatzschreiben sowie Übersetzungen, womit die Fähigkeiten der Prüfungsteilnehmer in Bezug auf Denken, Gedächtnis, Ausdrucksfähigkeit, Argumentation und Anwendung von Kenntnissen überprüft wurden. ${ }^{538}$ Die Aufgaben wurden zunächst von Studenten im Prüfungszentrum auf den Schwierigkeitsgrad hin getestet, gegebenenfalls verbessert, anschließend ausgedruckt und versiegelt. Inhalte und Themen waren landesweit vereinheitlicht, vor der Durchführung der Aufnahmeprüfung wurde das Prüfungszentrum abgesperrt und überwacht. Die zuständigen Lehrer und Professoren mussten sich bis zum Ende der Aufnahmeprüfung im Prüfungszentrum bleiben und durften nicht mit der Außenwelt in Kontakt treten. ${ }^{539}$

\footnotetext{
538 Vgl. Kuang Mei-jung 2004 S.75.

539 Vgl. Bildungsministerium (Hrsg.) 1974 S.735.
} 
Bei der Anmeldung zur Prüfung bekam jeder Teilnehmer eine Registernummer, dadurch wurde die Anonymität bewahrt. Die Teilnehmer durften ausschließlich Aufgaben, die mit ihrer Prüfungsnummer versehen waren, bearbeiten. Diese wurden dann anonym und von mindestens zwei Prüfern korrigiert. ${ }^{540}$

Der Anzahl an Prüfungsteilnehmern entsprechend wurden Plätze zum Bearbeiten der Aufgaben landesweit in unterschiedlichen Schulen eingerichtet, in zehn Prüfungskreisen, verteilt auf ganz Taiwan und die drei zugehörigen Inseln Penghu, Jinmen und Mazu. ${ }^{541}$ Der Prüfungstermin war auf den 2. Juli und die Zeit zur Lösung der Aufgaben in jedem Fach auf 80 Minuten festgelegt. Direkt nach der Prüfung wurden die Aufgabenlösungen in den Zeitungen als Beilage veröffentlicht, sodass die Prüfungsteilnehmer ihr Ergebnis einschätzen konnten. ${ }^{542}$

\subsubsection{Berechnungsmethoden der Prüfungsergebnisse und Verteilungsverfahren}

In jedem Fach war im Prinzip das Optimum von 100 Punkten zu erreichen, doch stellten einige Fachbereiche auch spezielle Anforderungen. So verlangten z.B. die geisteswissenschaftlichen Bereiche besondere Kenntnisse im Chinesischen und die naturwissenschaftlichen und sozialwissenschaftlichen Bereichen in Mathematik. $\mathrm{Ab}$ 1966 wurden Prüfungsergebnisse in diesen Fächern mit einer Gewichtung von 25\% berechnet, um die Anforderungen auch quantitativ zu belegen. ${ }^{543}$

Die Verteilung der Studienplätze lief folgendermaßen ab: Jedes Jahr bestimmte das Bildungsministerium die neu aufzunehmenden Studentenzahlen aller Fächer in allen

\footnotetext{
540 Vgl. ebd. S.736.

541 Vgl. ebd. S.734.

542 Vgl. ebd. S.735.

543 Vgl. ebd. S.734.
} 
Hochschuleinrichtungen. Die Prüfungsteilnehmer mussten mit der Anmeldung eine Rangliste mit ihren Wunschfächern abgeben.

Die Ergebnisse der sechs Prüfungsfächer wurden zunächst einzeln zusammengezählt, anschließend wurden die gesamten Prüfungsergebnisse aller Prüfungsteilnehmer gruppenweise arrangiert und in eine Rangordnung gebracht. Das Prüfungsergebnis, der Wunsch des Studienfaches und die Ranglisten der Prüfungsteilnehmer wurden miteinander abgeglichen und daraufhin der Studienplatz zugewiesen. ${ }^{544}$ Prüfungsteilnehmer mit besseren Ergebnissen wurde ihr Studienwunsch eher erfüllt. Wenn die Studienplätze allerdings besetzt waren, wurde der zweite Wunsch verfolgt usw. $^{545}$

\subsubsection{Sondermaßnahmen für bestimmte soziale Gruppen}

Bestimmte Gruppen erhielten aus politischen Überlegungen beim Aufnahmeprüfungsverfahren Vergünstigungen, etwa Angehörige von Minderheitenvölkern wie Tibetern und Mongolen oder von Minderheitengruppen aus Sinjing und Yunnan auf dem chinesischen Festland sowie Ureinwohner Taiwans. Die Erziehung jugendlicher Überseechinesen wurde in die Bildungsprojekte der US-Entwicklungshilfe mit einbezogen, und Taiwan wurde aufgefordert, für sie Studienplätze vor allem an den staatlichen Universitäten zu schaffen, sie erhielten nicht nur das Privileg eines Studienplatzes, sondern auch ein Stipendium. ${ }^{546}$ Zudem wurden den zwei Millionen Soldaten, die mit Chiang Kai-shek nach Taiwan gekommen waren und ab 1956 allmählich aus dem Dienst traten, zum Studium verholfen, ebenso jugendlichen Flüchtlingen aus kommunistischen Gebieten. Und weil die Kinder von Diplomaten

${ }^{544}$ Vgl. Kuang Mei-jung 2004 S.87.

${ }^{545}$ Vgl. ebd. S.68f.

${ }^{546}$ Vgl. Li Ching-erh 2003 und Chen Yueh-ping 2003. 
lange Zeit im Ausland gelebt hatten, wurde ihre Situation wie die der jugendlichen Überseechinesen gesehen. Sie alle hatten das Privileg, mit $10 \%$ bis $25 \%$ weniger Anforderungen als die übrigen Prüfungsteilnehmer im gewünschten Studienfach aufgenommen zu werden. Ab Mitte der 1960er-Jahre kamen jährlich ca. 25\% der aufgenommenen Studenten aus diesen Sondergruppen. ${ }^{547}$ In der Aufnahmeprüfung mussten sie aber zunächst beweisen, dass sie nicht nur die Lehrinhalte beherrschten, sondern auch, dass sie die geltenden gesellschaftlichen Normen und Erwartungen sowie die vom Staat erwünschte Ideologie akzeptierten. ${ }^{548}$ Erst dann bekamen sie eine Chance auf einen Studienplatz und damit zum sozialen Aufstieg.

\subsubsection{Die Erneuerungsphase (1972-1986)}

Die Anzahl der Prüfungsanmeldungen zur Hochschule war von 43.466 im Jahr 1965 auf 98.073 im Jahr 1973 massiv zugenommen; jedoch war der Anteil der aufgenommen von $39,13 \%$ zu $24,76 \%$ abgenommen. Die Zuwachsen der Prüfungsanmeldungen belastete auch die Durchführung der gesamten Aufnahmeprüfung zur Hochschule; zugleich wurde die Zunahme der Bildungsplätze gefordert. Zu Beginn der 1970er-Jahre wurde die Reform des Aufnahmeprüfungssystems zur Hochschule daher eingeleitet, nachdem in den „Plänen zur Ausbildung von Arbeitskräften“ mehr Studienplätze und eine Neuregulierung der Fächerstruktur anvisiert wurden. Die Maßnahmen für Erneuerung des Aufnahmeprüfungssystems zur Hochschule in dieser Phase waren folgend:

\subsubsection{Umorganisation des Aufnahmeprüfungssystems}

Um der Forderung nach individuellen und differenzierten Aufnahmeverfahren

\footnotetext{
547 Vgl. Bildungsministerium (Hrsg.) 1974, S.734 und Ting Shih-ta 2005, S.49f.

548 Vgl. Chen Li-fu 1973 S.10-16.
} 
nachzukommen, wurden zunächst Bildungsberater aus den USA nach Taiwan eingeladen, so Prof. Dr. Hsu Hsien-hsiu (1912-2001) ${ }^{549}$ der Purdrue Universität. Er übte Kritik am Aufnahmeprüfungsverfahren und unterbreitete folgende Reformvorschläge: Lehren und Lernen in der taiwanesischen Schule sei zu sehr in Richtung der Aufnahmeprüfung zur weiterführenden Bildung ausgerichtet, also nicht anders als im alten China, wo sich die Schüler auf die kaiserliche Beamtenprüfung vorbereiteten. Dr. Hsu empfahl, anstelle der bestehenden „Kommission zur Maßnahmenerstellung und Durchführung der gemeinschaftlichen Aufnahmeverfahren für die neuen Studenten zur Hochschule“ eine langfristig zuständige Aufnahmeprüfungskommission $\mathrm{zu}$ installieren. Weiterhin sollten die Aufnahmeverfahren von Universitäten und Fachschulen separat durchgeführt werden. Und da die Gliederung der Studienfächer sowie die Gruppierung der Prüfungsteilnehmer nicht differenziert genug sei, solle beides stärker untergliedert werden, um die unterschiedlichen Anforderungen den Kompetenzen der Prüfungsteilnehmer anzupassen. ${ }^{550}$

Zudem schlugen Dr. Hsu und Pädagogen wie Kuo Wie-fan und Tsung Liang-tung vor, das System der pädagogischen Diagnostik aus den USA - „Educational assessment and evaluation“ - inklusive Leistungstests, Begabungstests und Intelligenztests - zu übernehmen. Psychologische Tests sollten den Schülern helfen, eine vernünftige Entscheidung bei der Auswahl des Studienfaches oder Berufs zu treffen. Die

\footnotetext{
${ }^{549}$ Hsu Hsien-hsiu war Mathematik Professor und gründete 1961 die Fakultät für Mathematik an der Tsing Hua Universität in Taiwan. 1970 wurde er vom Chiang Ching-kou zur Berufung des Präsidenten an der Tsing Hua Universität in Taiwan eingelanden. Hsu erweiterte nicht nur die Fakultäten an der Tsing Hua Universität (1970-1975), sondern bemührte sich ab 1976 in der Nachbarschaft von Forschungsinstituten und den Universitäten Tsing Hua und Chioa Tung, ein nach dem Modell von Silicon Valley konzipierter Technologiepark in Hsinchu aufzubauen. Damit wurde die Ausweiterung privater Klein- und Mittelunternehmen stark unterstützt, vor allem in der Elektronik- und Computerindustrie. Vgl. Chang Chung-wei 2005.

${ }^{550} \mathrm{Vgl}$. Tsung Liang-tung 1986, S.609-657 und 687-692.
} 
individuelle Entwicklung sollte zudem in Schule und Familie besser unterstützt werden, um eine „blinde Kollektivhandlung ${ }^{\text {‘551 }}$ zu vermeiden. ${ }^{552}$ Um das Konzept in die Praxis umzusetzen, wurde der Erziehungsberater Dr. John S. Helmick, ein Mitarbeiter des „Educational Testing Service“ (ETS), aus den USA eingeladen, der den „Educational Testing Service“ mit entwickelt hatte. In der Schrift „University Entrance Examination Procedures. A Report to the Minister of Education of the Republic of China" machte er folgende Reformvorschläge in Bezug auf die Prüfungsaufgaben: ${ }^{553}$

(1) Die Korrektur der Aufgaben, die Berechnung der Ergebnisse und die Zuteilung der Studienplätze sollten mithilfe des Computers durchgeführt werden.

(2) Psychologen und Lehrer der Senior High Schools sollten an der Erstellung der Aufgaben teil haben, welche im „Multiple-Choice-Verfahren“ erstellt werden sollten.

(3) Die Aufnahmeprüfung zur Hochschule sollte von der Wissensüberprüfung (Achievement test) zu einen Fähigkeitstest (Aptitude test) umgewandelt werden.

Nachdem die taiwanesischen Pädagogen und Beamten über die Konzepte und Methoden in den USA für die Differenzierung im Bildungssystem sowie die pädagogische Diagnostik aufgeklärt worden waren, wurde ein „Bericht für die Verbesserung der Aufnahmeprüfung zur Universität“ von Tsung Liang-tung vorgelegt. $^{554}$

1972 traten die Fachschulen aus dem gemeinschaftlichen Prüfungsverfahren aus, und eine eigenständige Kommission regelte fortan die Aufnahme. 1976 wurde „die Kommission für die Aufnahmeprüfung zur Universität" errichtet, die erste dauerhafte

\footnotetext{
${ }^{551}$ In dieser Zeit wurde der Bildungseifer der Bevölkerung von Beamten und Wissenschaftlern als eine „blinde“ kollektive Handlung verstanden. In: Yang Kuo-shu und Ye Chi-cheng 1978 S.73-93.

${ }_{552}$ Vgl. Tsung Liang-tung 1986, S.626-629 und 635-638.

553 John S. Helmick 1972.

${ }^{554}$ Vgl. Tsung Liang-tung 1986, S. 719-761.
} 
Institution mit 15 bis 25 Mitgliedern. Sie war auch für die Verbesserung der Aufnahmeverfahren zuständig. ${ }^{555}$ Dazu entwickelte sie Reformmaßnahmen, wie eine neue Gruppierung der Prüfungsteilnehmer und neue Berechnungsmethoden der Prüfungsergebnisse mithilfe statistischer Verfahren. ${ }^{556}$

\subsubsection{2. Änderung von Anmeldung zur Prüfung und Zuteilung der Studienplätze}

Ab 1984 wurde eingeführt, dass die Prüfungsteilnehmer die Ranglisten ihrer Wunschfächer erst nach Erhalt der Prüfungsergebnisse ausfüllten. Außerdem wurden die Fakultäten nach zehn Kategorien differenziert und in vier Gruppen aufgeteilt:

Gruppe I: Geisteswissenschaften, Jura und Sozialwissenschaften, Handelswesen,

Gruppe II: Naturwissenschaften A und Technikwesen A,

Gruppe III: Naturwissenschaften B, Technikwesen B, Agrarwissenschaften B und Medizin,

Gruppe IV: Agrarwissenschaften A.

Die gemeinsamen Prüfungsfächer waren wie zuvor Chinesisch, „Drei Prinzipien des Volkes“ und Englisch, die Gruppenfächer der Gruppe I: Chinesische und Weltgeschichte, Chinesische und Weltgeographie sowie Mathematik für sozialwissenschaftliche Studenten; Gruppe II: Physik, Chemie und Mathematik für naturwissenschaftliche Studenten; Gruppe III - Physik, Chemie, Biologie und Mathematik für naturwissenschaftliche Studenten; Gruppe IV: Chemie, Biologie und Mathematik für naturwissenschaftliche Studenten. ${ }^{557}$

Damit wurden Geisteswissenschaften, Jura und Sozialwissenschaften sowie das Handelswesen wieder $\mathrm{zu}$ einer Gruppe zusammengestellt. Die

555 Vgl. Chiu Ai-ling 1998, S. 121-129.

556 Vgl. Tsung Liang-tung 1986, S. 630-635.

557 Vgl. Bildungsministerium (Hrsg.) 1984 S.159-163. 
naturwissenschaftlichen und technischen Fächer wurden hingegen mehr differenziert. Denn die Lehrinhalte von Mathematik und Naturwissenschaften wurden aus US-amerikanischen Lehrbüchern direkt übersetzt. Das war sehr lebensfern und schwer verständlich, sodass viele Schüler davor scheuten, diese Fächer zu wählen. ${ }^{558}$ Um die Anzahl der Bewerber für naturwissenschaftliche und technische Fächer zu erhöhen, wiesen die Beamten zum einen mehr Studienplätze aus, zum anderen versuchten sie zu verdeutlichen, dass die Prüfungsfächer Naturwissenschaften, Agrarwissenschaften und Technikwesen den Interessen und Begabungen mancher Schüler entgegenkamen. Durch die Änderung erfuhr die naturwissenschaftliche und technische Erziehung in der Schule allmählich mehr Wertschätzung, und immer mehr Schüler gaben sich in den entsprechenden Fächern mehr Mühe, um später die Aufnahmeprüfung bestehen zu können.

\subsubsection{Neue Aufgabenstellung und Neubewertung der Prüfungsergebnisse}

Ab 1973 wurden die Korrektur der Prüfungsaufgaben, die Berechnung der Ergebnisse, die Anmeldung zur Prüfung und die Verteilung der Studienplätze mithilfe des Computers durchgeführt. Damit änderten sich auch die Prüfungsaufgaben: Aufsatzschreiben, Argumentation und Lückentexte wurden massiv reduziert und durch „Multiple-Choice-Fragen“ ersetzt. Folgend stellten die schuliche Didakik und Lehrinhalte auf diese Formen der Prüfungsaufgaben, daher befürchtete die Regierung, dass sich die Ausdrucksfähigkeit der Schüler dadurch reduziert, deswegen wurden die Argumentationen im Fach „San-min chu-i“ ab 1976 ; das Aufsatzschreiben und die Übersetzung in den Fächern Englisch und Chinesisch ab 1982 erneuert praktiziert. ${ }^{559}$

\footnotetext{
$558 \mathrm{Vgl}$. 6.3.5.2. in dieser Arbeit.

559 Vgl. Chiu Ai-ling 1998, S.293.
} 
Ab 1975 konnte jede Fächergruppe besondere Anforderungen an Studienplatzbewerber stellen, so z.B. in der Gruppe B in den Fächern Chinesisch und Englisch und in Gruppe A im Fach Mathematik. Dabei wurden die durchschnittlichen Punktzahlen aller Prüfungsteilnehmer einer Gruppe bzw. der besseren Hälfte der Gruppe als Basis der Anforderungen für die Bewerbung um einen Studienplatz in einem bestimmten Fach verwendet. ${ }^{50}$ Ab 1984 durfte jede Fächergruppe ihre besonderen Anforderungen an die Leistungsfächer der Bewerber und die Gewichtung dieser Fächer selbst festsetzen. Damit sollte jede Fakultät ihre wünschenden Bildungsbewerber aussuchen können.

1973 wurde die Richtlinie für pädagogische Diagnostik und Beratung in den Senior High School eingelassen. Der Zweck dieser Richtlinie war folgend, dass die Schüler in den Senior High School mithlife der pädagogische Diagnostik und Beratung ihre Entscheidung für künftigen Bildungsweg richtig treffen konnten. ${ }^{561} 1975$ wurde ein „Itembank für die Erstellung der Prüfungsaufgaben in Bezug auf die Senior High School“ von Fachlehrern aus neun Fachbereichen der Senior High School durchgeführt. Dies schaffte eine neue Basis zur Erstellung der Prüfungsaufgaben für die Aufnahmeprüfung zu den Universitäten. ${ }^{562}$

\footnotetext{
560 Vgl. Chiu Ai-ling 1998, S.130.

561 Vgl. Tsung Liang-tung 1986, S. 635-638.

562 Vgl. ebd. S. 651-653.
} 


\section{Der Kampf um einen Studienplatz}

Für einen erfolgreichen Übergang in den Arbeitsmarkt stellen Schulabschlüsse und berufliche Ausbildung entscheidende biografische Weichenstellungen dar. Sehr viele Eltern in Taiwan strebten deshalb eine höhere Bildung ihrer Kinder an. 1953 wurde der Wunsch nach Verbesserung der beruflichen und sozialen Position sowie des Einkommens von Chiang Kai-shek als mangelnde Rücksicht auf die Gesamtentwicklung des Landes kritisiert. Seitdem wurde „Sheng-hsueh chu-i““563 zu einem wesentlichen Punkt der Bildungspolitik. Schon zu Beginn der Ausweitung der Berufsschulen in den 1950er-Jahren entstanden Meinungskonflikte zwischen den zuständigen Beamten und Eltern bzw. Schülern. Da die Berufsschule allein der Ausbildung und nicht der Vorbereitung auf eine weiterführende Bildung dienen sollte, befürchteten viele Eltern, dass ihre Kinder durch den Besuch der Berufsschule die Chance zum künftigen sozialen Aufstieg verlören.

Die „Arbeitsgruppe Arbeitskräfteplanung“ argumentierte 1970, dass aufgrund der konfuzianischen Tradition alle Schularten ihren Schwerpunkt auf die Vermittlung des Wissens und auf die Vorbereitung für die Aufnahmeprüfung legen. Dadurch werde die allgemeine moralische Erziehung vernachlässigt. „Außerdem stellt sich die Frage, was die Schüler in der Schule gelernt haben, ob sie sich damit in der Zukunft um eine passende Stelle bewerben und das erworbene Schulwissen bei der Arbeit verwenden können ... Über solche Fragen machen sich Schule, Eltern und Schüler gar keine Gedanken." Büroarbeit werde zu hoch bewertet und die technische oder körperliche Arbeit geringgeschätzt. ${ }^{\text {(5564 }}$

\footnotetext{
$563 \mathrm{Vgl}$. 6.3.1. in dieser Arbeit.

564 Zit. nach Hwang Tsung-mu 2007, S.32. Eigene Übersetzung.
} 
Nach dem Verständnis der Beamten war die vom Konfuzianismus geprägte Einstellung zur Erziehung sowie zur Arbeit noch immer die Ursache für das Streben nach Bildungstiteln sowie für die Geringschätzung naturwissenschaftlicher und technischer Fachausbildungen. Doch fehlte es laut den „Vierjahresplänen zum wirtschaftlichen Aufbau“ gerade im Bereich Technik an entsprechenden Arbeitskräften. Solange der Großteil der Bevölkerung nach Höherer Bildung strebte, entstehe auf Dauer ein unausgeglichenes Verhältnis zwischen dem Bedarf des Arbeitsmarktes und der Zahl der Absolventen. Zur Lösung der Diskrepanz wurde die Vergabe der Bildungsplätze staatlich kontrolliert, und die Bildungsbewerber wurden in Richtung einer beruflichen Ausbildung gelenkt. Die Beamten drängten darauf, dass bei der Schul- sowie der Studienfachwahl die künftigen Beschäftigungsmöglichkeiten berücksichtigen wurden. Die Jugendliche sollten erkennen, dass etwa eine naturwissenschaftliche oder technische Fachausbildung mehr Chancen auf eine künftige Stelle in der Arbeitswelt böte als ein geistes- oder sozialwissenschaftliches Studium. Diese Aufforderungen wurden mit der neuen Regulierung der Bildungsplätze und der Reform des Aufnahmeprüfungssystems reagiert.

\subsection{Konkurrenz um Bildungsplätze}

Dass die Teilnehmer der Aufnahmeprüfung gemäß den „Plänen zur Ausbildung von Arbeitskräften“ gezielt in Ausbildungen solcher Bereichen gelenkt wurden, wo ein Bedarf am Arbeitsmarkt bestand, und dementsprechend auf weiterführende Schulen und Universitäten verteilt wurden, hatte für die schulische Realität Konsequenzen.

\subsubsection{Qualitative Abstufungen bei gleicher Schulform}

Jedes Jahr stand nur für ein knappes Drittel der Absolventen ein Studienplatz zur 
Verfügung. Der Forschungsbericht des „Council for Economic Planning and Development“ $1982^{565}$ zeigt, dass deshalb eine steigende Zahl an Bewerbern die Aufnahmeprüfung nicht bestand und versuchte, diese zu wiederholen. Daneben bestand die Möglichkeit, eine andere Form der Hochschule, etwa die zwei- oder dreijährige Fachschule, zu besuchen oder eine Arbeit aufnehmen.

\begin{tabular}{|c|c|c|c|c|c|c|c|c|}
\hline \multirow[b]{3}{*}{$\begin{array}{l}\text { Anzahl der } \\
\text { Angemeldeten }\end{array}$} & Senior High & $\begin{array}{l}\text { Non- Senior } \\
\text { High }\end{array}$ & \multicolumn{4}{|c|}{ Senior High Schools } & \multicolumn{2}{|c|}{ Non-Senior High Schools } \\
\hline & \multicolumn{2}{|c|}{$\begin{array}{l}\text { Gesamte Anzahl } \\
\text { der Prüfungsteilnehmer }\end{array}$} & $\begin{array}{l}\text { Absolventen } \\
\text { in diesem } \\
\text { Jahrgang }\end{array}$ & $\begin{array}{l}\text { Wiederho } \\
\text { lende }\end{array}$ & $\begin{array}{l}\text { Öffentliche } \\
\text { Schulen }\end{array}$ & $\begin{array}{l}\text { Private } \\
\text { Schulen }\end{array}$ & $\begin{array}{l}\text { Absolventen } \\
\text { in diesem } \\
\text { Jahrgang }\end{array}$ & Wiederholend \\
\hline & 84.178 & 13.005 & 44.268 & 39.910 & 67.224 & 16.954 & 4.301 & 8.704 \\
\hline Gesamtanzahl & \multicolumn{2}{|l|}{97.183} & \multicolumn{2}{|l|}{84.178} & \multicolumn{2}{|l|}{84.178} & \multicolumn{2}{|l|}{13.005} \\
\hline \multirow[t]{2}{*}{$\begin{array}{l}\text { Anteil der } \\
\text { Angemeldeten }\end{array}$} & $86,6 \%$ & $13,3 \%$ & $52,6 \%$ & $47,4 \%$ & $79,9 \%$ & $20,1 \%$ & $33,1 \%$ & $66,9 \%$ \\
\hline & \multicolumn{2}{|l|}{$100 \%$} & \multicolumn{2}{|l|}{$100 \%$} & \multicolumn{2}{|l|}{$100 \%$} & \multicolumn{2}{|l|}{$100 \%$} \\
\hline $\begin{array}{l}\text { Anzahl der } \\
\text { Aufgenommenen }\end{array}$ & 27.003 & 1.405 & 14.651 & 12.352 & 24.289 & 2.714 & 688 & 725 \\
\hline Gesamtanzahl & \multicolumn{2}{|l|}{28.408} & \multicolumn{2}{|l|}{27.003} & \multicolumn{2}{|l|}{27.003} & \multicolumn{2}{|l|}{1.413} \\
\hline \multirow[t]{2}{*}{$\begin{array}{l}\text { Anteil der } \\
\text { Aufgenommenen }\end{array}$} & $95,0 \%$ & $5,0 \%$ & $54,3 \%$ & $45,7 \%$ & $90,0 \%$ & $10,0 \%$ & $48,7 \%$ & $51,3 \%$ \\
\hline & \multicolumn{2}{|l|}{$100 \%$} & \multicolumn{2}{|l|}{$100 \%$} & \multicolumn{2}{|l|}{$100 \%$} & \multicolumn{2}{|l|}{$100 \%$} \\
\hline
\end{tabular}

Laut Bericht des CEPD 1982 gab es 97.183 Anmeldungen zur Universität im Jahr 1981, daunter konnte nur 29,23\% aufgenommen werden. Es kamen 86,6\%, also der Großteil der aufgenommen Prüfungsteilnehmer aus den Senior High Schools 841.778 -; 47,4\% der Anmeldungen aus den Senior High Schools (39.910 von 84.178) hatten schon mindestens einmal die Aufnahmeprüfung abgelegt, 45,7\% der aufgenommen Prüfungsteilnehmer aus den Senior High Schools waren Prüfungswiederholer. 20,1\% der Anmeldungen der Senior High Schools kamen aus 
den privaten Senior High Schools, 10,0\% der aufgenommen Prüfungsteilnehmer aus den Senior High Schools waren Schüler der privaten Senior High Schools. Nur ein kleinerer Anteil von 13,3\% der Prüfungsanmeldungen kam aus Senior Vocational High Schools, Förderschulen oder fünfjährigen Fachschulen - 13.005 -. Darunter hatten $66,9 \%$ der Bewerber schon mindestens ein Mal die Prüfung geschrieben. Gegenüber den Bewerbern aus den Senior High Schools hatte diese Schüler sehr geringe Chancen, nur 5\% erhielten einen Studienplatz.

Im diesen Bericht zeigt eine wietere Analyse. Von 195 Senior High Schools lag die Erfolgsquote bei 28 Schulen über 40,1\%, was einen Anteil von $64 \%$ an der Gesamtzahl der aufgenommenen Studenten ausmachte. 53 Schulen, in denen die Erfolgsquote zwischen 10,1\% bis $40 \%$ lag, erreichten einen Anteil von $25,4 \%$ an der Gesamtzahl. Nur $0,1 \%$ bis $10,0 \%$ der Absolventen aus 80 Schulen konnten sich für einen Studienplatz qualifizieren, was $4,1 \%$ der Gesamtzahl ausmacht. Die Absolventen von 34 Schulen dagegen wurden in keiner Universität aufgenommen. Also nur die Absolventen aus den oben erwähnten 28 Senior High Schools bzw. gerade $14,4 \%$ der gesamten Senior High Schools - diese Schulen liegen meistens in den Großstädten - hatten eine gute Chance, einen Studienplatz zugewiesen zu bekommen. ${ }^{566}$

Das Forschungsinstitut für Erziehung an der „National Taiwan Normal University“ wies zudem darauf hin, dass 92,02\% der Absolventen der 20 sogenannten „Star Senior High Schools“ im Jahr 1991 einen Studienplatz erhalten hatten, darunter waren 13 Schulen mit einer Erfolgsquote von über 78\%. ${ }^{567}$ Zur „Star Senior High

566 CEPD 1982, S. 136

567 Vgl. Center for Educational Research NTNU 1994, S.63. 
School“" wurde eine Schule erklärt, wenn ein Großteil der Absolventen die Aufnahmeprüfung zur Hochschule bestand. Sie genoss daraufhin einen besseren Ruf und wurde zur beliebtesten Schule innerhalb des Prüfungsbezirkes. Diese Forschungsergebnisse zeigen, dass nur deren Absolventen mit großer Wahrscheinlichkeit die Universität besuchen durften, wobei die Wahrscheinlichkeit jährlich noch weiter stieg. Dies deutet darauf hin, dass bei der Auslese innerhalb scheinbar gleichwertiger Schulen ein „Teufelskreis“ entstanden war: Die „Star Senior High Schools“ zogen immer mehr leistungsstarke Bewerber und spätere Prüfungsteilnehmer an, während aus den normalen Senior High Schools nur Bewerber mit schlechteren Leistungen die Aufnahmeprüfung ablegten und logischerweise häufiger durchfielen. Daraufhin wurden sogar einige Schulen geschlossen oder in andere Schulformen, z. B. berufliche Schulen oder Ausbildungszentren, umgewandelt.

Dass die soziale Anerkennung einer beruflichen Ausbildung nach wie vor gering war, lag indes nicht nur an der traditionellen Bildungseinstellung, den schmalen Subventionen des Staates, sondern auch an der Reihenfolge der Aufnahmeprüfungen. Das Bildungssystem fungierte als die zentrale Zuteilungsstelle für soziale Chancen und wurde damit langsam zum „Leistungssieb“ der Nation, der US-amerikanische vergleichende Erziehungswissenschaftler Douglas C. Smith bezeichnete das Phänomen als „Academic Darwinism““.568 Vor allem in der Sekundarstufe machten sich die Schüler untereinander Konkurrenz, weil ihre Lernleistung ständig in Ranglisten abgebildet wurde - in der Klasse, in der Schule, später in den Bezirken (Aufnahmeprüfung zur Sekundarstufe II) sowie im ganzen Land (Aufnahmeprüfung zur Hochschule). Die Aufnahmeprüfung zur Sekundarstufe II fand in der Regel zunächst zur Senior High School statt, dann zur fünfjährigen Fachschule und danach 
zur Senior Vocational High School. Das bedeutete, dass die Senior High Schools Priorität besaßen und die Absolventen aus den Junior High Schools auswählen konnten, wogegen die Fachschulen und Senior Vocational High Schools oft nur Bewerber mit relativ schwacher Schulleistung bekamen. Senior High Schools, Senior Vocational High Schools, Fachschulen und auch Universitäten sowie Fachhochschulen wurden nach den Prüfungsergebnissen der Teilnehmer in eine Rangfolge gebracht. Das strenge Leistungsprinzip verschärfte enorm die Konkurrenz um Bildungschancen, denn die Schüler strebten nicht einfach nur nach einem Studienplatz, sondern nach einem Platz an einer „guten Schule“, einer „guten Universität“ sowie in einem ,guten Fach“. Sie mussten deshalb nicht nur erfolgreich die Prüfung ablegen, sondern auch allerbeste Leistungen erbringen. Mit der neu eingeführten Testform der „Multiple-Choice-Fragen“ wurden die Prüfungsteilnehmer noch feiner differenziert: Ein winziger Unterschied in der Punktzahl konnte sehr große Konsequenzen für die weitere Bildungslaufbahn haben.

Die Schulen bemühten sich darum, nur Schüler mit besten Leistungen aufzunehmen, um ihr Ansehen in der Gemeinde zu heben. Dies hatte bereits Auswirkungen auf Schüler der Junior High Schools, die nach ihren Ergebnissen in Intelligenztests und schulischen Leistung eingruppiert wurden, sogar Lehrer wurden aufgrund der Leistungen ihrer Schüler eingestuft. Daraus ergaben sich sog. „Starschulen“, „Starlehrer“ und „leistungsstarke Klassen“ oder Sheng-hsueh-Klassen bzw. „leistungsschwache Klassen“, auch „Buffalo-boy-“ oder „Girl-Klassen“). Obwohl das taiwanesische Bildungsministerium eine heterogene Verteilung bei der Eingruppierung der Schüler forderte, wandte der größte Teil der Schulen eine homogene Verteilung gemäß dem Leistungsprinzip an, um den Unterrichts effektiver durchführen zu können. 


\subsubsection{Durch Tests bestimmtes Schulleben}

Hung Chen-tsung, ein Abgeordneter der Provinzversammlung, berichtet 1981: „In der regulären Junior High School gibt es sogenannte Kapiteltests, Tests in der Mitte des Semesters, Tests am Ende des Semesters, Wochentests, Gruppierungstests, Wahltests, Wiederholungstests, analoge Aufnahmeprüfungen, Wettbewerbtests usw.“569 In der Junior High School mit hohem Prestige würden 18 Tests innerhalb einer Woche, 324 bis 360 Tests innerhalb eines Semesters durchgeführt. Die Schüler seien durch die zahllosen Prüfungen und den Leistungsdruck stark belastet, denn viele Lehrer organisierten Unterricht bzw. Nachhilfe außerhalb des offiziellen Stundenplans, nach der Schule und sogar am Wochenende, um die Prüfungsaufgaben einzuüben. Grundschüler gingen durchschnittlich fünf Stunden pro Woche zur Nachhilfe, um ihre Leistung in der Schule zu verbessern oder eine bestimmte Begabung zu vertiefen.

Die Schüler der Junior High Schools durchschnittlich zwölf Wochenstunden die Nachhilfe. ${ }^{570} 51,8 \%$ der Lehrer gaben zu Hause Nachhilfeunterricht; 30,1\% bei einem Nachhilfeinstitut. ${ }^{571}$ Die Nachhilfe diente meist dazu, Lerninhalte wurden auswendig zu lernen bzw. einzupauken, die Technik der Aufgabenlösung einzuüben und die schulische Leistung insgesamt zu verbessern.

\subsection{Kritik am ,blinden Streben nach höherer Bildung“6}

Zwar hatten die Schulkinder schon seit den 1950er-Jahren in der Grundschule und ab

Mitte der 1960er-Jahre in der Junior High School enorm unter Leistungsdruck und Prüfungsstress gelitten, sodass immer wieder Forderungen nach einer Bildungsreform laut wurden, um die Schüler zu entlasten. In den 1970er- und 1980er-Jahren wurden

\footnotetext{
569 Vgl. 1981-06-02/ United Daily News /S.03/

570 Vgl. Die Welt 1996 S.51.

571 Vgl. ebd. S.53.
} 
die Phänomen der schulischen Erziehung vom taiwanesischen Bildungsministerium selbst als „krankhaftes Phänomen“ gesehen. Im Aufsatz „Erziehungsprobleme unter Sheng-hsueh chu-i“ (1978) bezeichneten der Psychologe Yang Kuo-shu und der Soziologe Ye Chi-chen, beide vom US-Liberalismus beeinflusst, ${ }^{572}$ „Sheng-hsueh chu-i“" als blindes Streben nach weiterführender Bildung, wodurch die Betreffenden „eigene Fähigkeiten, Persönlichkeit, Interesse, Motivation und Finanzzustand" vernachlässigten und nicht mehr darüber nachdachten, wie man durch die schulische Bildung der Gesellschaft und auch sich selber zu Diensten sein konnte. Höhere Bildung werde dadurch ganz rational zum Mittel, wodurch man sich bessere Zukunftschancen erwarb, ohne zu berücksichtigen, ob man geeignet sei und welche Form der Ausbildung oder welches Fach besser zu einem passe. Deshalb sei das Streben nach weiterführender Bildung nur „ein blindes Nachfolgen und Mitmachen“. „Die Folge war, dass die weiterführende Bildung zum Selbstzweck wurde und man nicht mehr danach fragte, aus welchem Grund man sich um die weiterführende Bildung bemühte. ${ }^{6573}$ Die beiden Wissenschaftler verfolgten ein anderes Bildungsideal als Chiang Kai-shek, nämlich dass schulische Erziehung dem Zweck der Selbsterkenntnis und der persönlichen Entfaltung dienen solle. Dem stehe das Phänomen „Sheng-hsueh chu-i“ als unreflektierte kollektive Handlung gegenüber, was nur durch die Unterstützung zu einer individuellen Entwicklung, d.h. durch eine Erziehung zu Selbstständigkeit und Selbstbewusstsein in Schule und Familie geändert werden könne.

$\mathrm{Da}$ in den 1970er- und 80er-Jahren das mithilfe des Konzepts „Human Resources“ gestaltete Bildungswesen ${ }^{574}$ und das Streben der Bevölkerung nach

572 Beide Wissenschaftler der National Taiwan University hatten in den USA studiert.

573 Zit. nach Yang Kuo-shu und Ye Chi-cheng 1978 S.75f. Eigene Übersetzung.

574 Vgl. Li Kou-ting und Chen Za-mu 1987, S. 481-486. 
höherer Bildung unter Einfluss der konfuzianischen Tradition ${ }^{575}$ als wichtige

Faktoren für Industrialisierung und Wirtschaftsaufschwung gesehen wurden und Taiwan zudem in dieser Zeit von der internationalen Gemeinschaft isoliert war, zögerte die KMT-Regierung, eine Bildungsreform durchzuführen, um die Stabilität ihrer Herrschaft und das Wirtschaftswachstum nicht zu gefährden.

\subsection{Das Scheitern der Bildungsreform von 1996}

Nach Aufhebung des Kriegsrechts 1987 wurde nun sowohl vonseiten der Regierung als auch der Öffentlichkeit das Problem von „Sheng-hsueh chu-i“ als wesentliche Frage der Erziehung gesehen. Die Initiativgruppe der Bildungsreform stellte 1994 vier Forderungen an das Bildungsministerium: die Förderung neuer Senior High Schools und Universitäten, die Anzahl an Schulklassen und Schulen zu verkleinern, die Modernisierung der schulischen Erziehung einzuleiten und ein Bildungsgesetz zu entwerfen. ${ }^{576}$ Themen wie Aufsicht über die Schule, Maßnahmen der Differenzierung durch ein einheitliches und gemeinschaftliches Aufnahmeprüfungssystem und die Regulierung der Bildungsplätze nach den „Plänen zur Ausbildung von Arbeitskräften“ wurden kritisch behandelt und eine „schülerzentrierte“ und lebensnahe Idee für die schulische Evaluation und eine Curriculum als Ziel erklärt. ${ }^{577}$ Die Mitglieder des Kommissionsrats für eine Bildungsreform forderten die „Deregulierung، ${ }^{678}$ und Liberalisierung der bestehenden Bildungspolitik durch die

\footnotetext{
575 Vgl. Peter Berger 1984 S.14-18; King Yeo-chi 1985; Yu Ying-shih 1986 und Hwang Kwang-Kuo 1983 und 1988 S.276-295.

576 Vgl. Hsueh Hsiao-hua 1996 S. 267-281.

577 Vgl. z. B. Chang Ching-hsi und Wu Hui-ling 1996; Wang Jenn-wu und Ling Wen-ing 1996 (a) und (b); Chu Ching-i, Tai Hua und Chen Chih-chia 1995.

578 „Deregulierung“ ist ein Begriff für die Förderung der Liberalisierung der Märkte aus dem wirtschaftlichen Bereich. Begründet wird Deregulierung mit negativen mikro- und makroökonomischen Folgen staatlicher Regulierung, die wirtschaftliche Aktivitäten verhindern oder in eine falsche Richtung lenken. Aus wettbewerbspolitischer Sicht soll mit Hilfe von Deregulierungen eine Beseitigung von marktwirtschaftlichen Verzerrungen erreicht werden. Durch die Deregulierung wurde die bisherige staatliche Kontrolle und Regulierung der Bildungsangebote sichtbar. Zugleich 
Berücksichtigung ihrer Reformvorschläge. Ab 1996 wurden die Vorschläge zur

Bildungsreform umgesetzt, d.h. mehr Senior High Schools einzurichten und mehr

Studienplätze zu schaffen. Zugleich sollten Aufnahmeprüfungen zur weiterführenden

Bildung zwei Mal pro Jahr stattfinden und die Prüfungsinhalte vereinfacht werden. ${ }^{579}$

Doch neun Jahre später äußerte Bildungsminister Tu Cheng-sheng in einem Interview im Juli 2005, dass die „,verderbliche Spur“ des „Sheng-hsueh chu-i“ die Ziele der Bildungsreform verhindert habe. ${ }^{580}$ Auch Lee Yuan-tseh, der Präsident des Kommissionsrats, entschuldigte sich beim Parlament, dass die Bildungsreform gescheitert sei, weil der auf den Schülern lastende Leistungsdruck nicht gemindert, sondern sogar erhöht worden sei. Das Ergebnis stand dem Ziel der Bildungsreform folglich völlig entgegen. Die Strategie der Deregulierung und Liberalisierung der Bildung hatten „Sheng-hsueh chu-i“ gegenüber nicht viel bewirkt.

wurde diskutiert, in welche Bereiche der Staat sich einmischen sollte, um die Gerechtigkeit der Verteilung der Mittel aus staatlichem Haushalt zu sichern.

${ }^{579}$ Im herkömmlichen Aufnahmesystem wurden die Durchführung der Aufnahmeprüfung und die Zuteilung der Bildungsplätze verbunden. Aufgrund des „Multiprogramms zur Aufnahme der neuen Schüler zur Senior High School und zur Universität“" wurde die herkömmliche Aufnahmeprüfung innerhalb eines Prüfungskreises durch einen landesweiten „,Test der Basiskenntnisse“ ersetzt. Jedes Jahr fanden zweimal „Tests der Basiskenntnisse“ statt, der erste im Juni und der zweite im Juli landesweit gleichzeitig. Der Zweck der zweimaligen Durchführung der Prüfungen war, den Nachteil der herkömmlichen einmaligen Aufnahmeprüfung zu vermeiden, z. B. dass die Schüler wegen Nervosität die Aufgaben nicht so gut lösen wie im normalen Zustand. Der Zweck des „Tests der Basiskenntnisse“ lag darin, den Lernstandes der Schüler zum besten, jedoch spielte das Ergebnis dieser Tests nur zu einem geringen Teil der Rolle für den Eintritt zur weiterführenden Bildung, denn es wurde eine neue Methode für den Zugang zur weiterführenden Bildung entwickelt, der Bewerbungsweg. Die schulische Leistung sowie schulische und außerschulische Aktivitäten der Bewerber wurden in diesen Weg einbezogen. Im „Test der Basiskenntnisse“ blieben die Prüfungsfächer unverändert, jedoch wurde Prüfungsfach Sozialkunde und die „Kenntnis über Taiwan“ - Heimatkunde - hinzugefügt und das Fach „Drei Prinzipien des Volkes“ gestrichen.

${ }^{580}$ Vgl. Chinatimes Weekly, Interview mit Tu Cheng-sheng am 11.07.2005. 


\section{Schlussbetrachtung}

Der Konfuzianismus war nicht nur seit lange die donimaten Führung des chinesischen Kaiserreichs. Sonder auch bevor die westlichen Bildungskonzepte in Taiwan richtunggebend wurden, waren Erziehung und Bildung stark vom Konfuzianismus geprägt. Nach der konfuzianischen Lehre basiert die hierarchische Gesellschaft auf der Leistung sowie der Persönlichkeit des Einzelnen. Die Aufgabe der Bildung bestand solcherart primär in der Kultivierung, vor allem der Verfeinerung der Umgangsformen, sowie darin, die jahrtausendealte Kultur Chinas zu überliefern, um Staatsdiener und soziale Führungspersonen auszubilden. Die Schüler wurden dazu angehalten, unablässig nach Optimierung der Persönlichkeit zu streben und ihre moralischen und kognitiven Qualitäten zu verbessern, um später soziale Verantwortung übernehmen zu können. Das Erziehungsziel fokussierte den Gebildeten oder „Edlen“, der sich nicht mit niederen Tätigkeiten, z. B. Handwerk, Technik oder Geschäftsführung, beschäftigte. Die Intention bestand also vorderhand nicht darin - wie bei der Erziehung in westlichen Kulturen -, Fachkräfte im Dienst der sozialen und wirtschaftlichen Entwicklung des Landes auszubilden, zu religiösem Glauben oder auch Nationalbewusstsein zu erziehen. Vielmehr wurde die im Westen übliche, auf die Ausübung eines Berufs ausgerichtete Ausbildung als „weltlich“ abgelehnt, und naturwissenschaftliche oder technische Bildung fand in der traditionellen konfuzianischen Erziehung kaum Berücksichtigung. Fächer wie Mathematik, Naturwissenschaft, Geographie, Sport, Musik und Zeichnen waren folglich nicht Gegenstand des Unterrichts.

Auch das im 7. Jahrhundert in China eingeführte kaiserliche Beamtenprüfungssystem vermittelte in erster Linie konfuzianische Werte und Inhalte. Im Allgemein war die kaiserliche Beamtenprüfung ein Wettbewerben in Aufsatzschreiben, Argumenten und 
Dichten bzw. Grammatik, Rhetorik, Logik und Kalligraphie usw.. Die Aneignung der Klassiker bzw. moralischen und politischen Führungen des Kaiserreichs und Beherrschung der Kulturtechnik wie Schreiben des „achtgliedrigen Aufsatzes“, Dichten, Kalligraphie usw. waren die Voraussetzung für Eintretten auf die herrschende Schichte. Es motivierte den Großteil der Bevölkerung dazu, sich mit der konfuzianischen Lehre zu beschäftigen, denn nach erfolgreichem Bestehen der Prüfung ermöglichte es den sozialen Aufstieg in den öffentlichen Dienst, ohne Berücksichtigung von Herkunft und sozialem Stand - ein zwar schwieriger, aber vergleichsweise schneller Weg in der feudalistischen Zeit Chinas. Das kaiserliche Beamtenprüfungssystem diente einerseits dazu, die intelligentesten und tüchtigsten Personen des Kaiserreichs auszuwählen, um sie als Verwaltungskräfte auszubilden, andererseits sorgte die zentral vom Staat gesteuerte und einheitliche Zulassungsprüfung dafür, das Gedankengut der Bewerber zu vereinheitlichen und zu kontrollieren, um die feudale Herrschaft zu festigen. Das chinesische Kaiserreich bestimmte als „Dirigent“ der Staatsideologie die Prüfungsinhalte und fungierte als Regulator und Wahrer der Gerechtigkeit bei der Durchführung - jedoch nicht als Investor, um die Bildung seiner Bevölkerung zu garantieren, denn in der Regel war die höhere Bildung dem Nachwuchs der gehobenen Schichten vorbehalten, während die elementare Erziehung eine Angelegenheit der lokalen Gemeinden sowie von einzelnen privaten Initiativen blieb.

Die Angleichung an das westliche Modell einer vom Staat geförderten und kontrollierten Bildung und Erziehung wurde in Taiwan erst im Zuge der Modernisierungsbewegung vorangetrieben, zuerst vom chinesischen Kaiserreich, in der Besetzungszeit vom japanischen Kaiserreich und schließlich vom KMT-Regime. Während die Beweggründe zur Modernisierung in der westeuropäischen Kultur 
ursprünglich auf Abgrenzungsbestrebungen zum unaufgeklärten, „dunklen“ Mittelalter zurückzuführen sind, zielten innovative Ideen im 19. und 20. Jahrhundert primär auf eine Veränderung der Gesellschaft - zunächst in Richtung eines Nationalstaates, dann auf Demokratie und Industrialisierung. In den fernöstlichen Ländern wie China, Japan oder auch Taiwan unterlagen die Bestrebungen indes äußerem Zwang im Zuge der Ausweiterung des westlichen Imperialismus. Hier wurde die Modernisierung durch Auseinandersetzungen zwischen westlichen bzw. exogenen und traditionellen bzw. endogenen Modellen sowie durch Widerstände und Forderungen begleitet und war somit einem Lernprozess unterworfen, den die Bevölkerung zunächst durch Laufen musste.

In der ersten Hälfte des 17. Jahrhunderts waren die Bildungskampagne der katholischen und calvinistischen Missionen aktiv Taiwanesen zum Christen zu bekehren. Nach der Harrschaft des Ming- und Ching-Kaiserreichs über Taiwan wurden die konfuzianische Erziehung und das kaiserliche Beamtenprüfungssystem in Taiwan eingeführt. Nach der Konstitution der Provinz Taiwan 1885 wurden die westlichen Bildungseinrichtungen im Zusammenhang mit der Selbstärkungsbewegung errichtet. Doch während der Kolonialherrschaft Japans wurde die Erziehungslandschaft hin zu modernen Schulen völlig umgewandelt, was sowohl der Integration als auch der Ausbreitung des japanischen Imperialismus bzw. der Kriegsvorbereitung diente. Die modernen Bildungseinrichtungen verbesserten deutlich die Erziehungsqualität im Vergleich zu den konfuzianischen Schulen, die Lehrinhalte wandelten sich von konfuzianischen Klassikern zur Vermittlung von Kenntnissen nach modernem Maßstab. Die vom Gouvernement Taiwan errichteten Lehrerausbildungsanstalten bildeten die Basis zur Ausbildung von qualifizierten Lehrkräften für schulische Erziehung unter der Aufsicht der Kolonialherrschaft. Die 
Verbreitung der allgemeinen Elementarbildung bei Rückgang der konfuzianischen Lehre bedeutete aber nicht nur eine bessere Schulbildung, sondern auch dass die Taiwanesen ihrer kulturellen Identität beraubt wurden. Stattdessen rückte die japanische Sprache, Kultur sowie japanische Sitten und Gebräuche in den Vordergrund, und Taiwanesen wurden zu loyalen Untertanen des japanischen Kaisers erzogen. Dazu wurde die breite elementare Bildung gefördert, der Zugang zur höheren Bildung war für taiwanesische Jugendliche allerdings sehr eingeschränkt. Die während der japanischen Besatzungszeit aufgebauten Bildungseinrichtungen lieferten später die Grundlage für die Bildungskonzepte des nachfolgenden KMT-Regimes.

Die Annäherung an westliche Bildungskonzepte in China zwischen 1861 bis 1949 wurde von den Gelehrten als Fortschritt und Allheilmittel „zur Rettung des Landes“" verstanden, durch das China reich und mächtig bzw. konkurrenzfähig werden konnte. Darüber hinaus war die Politik zugunsten eines modernen staatlichen Bildungswesens stark von internationalen Beziehungen beeinflusst. Der Prozess verlief von der auf konfuzianischem Gedankengut basierenden Ablehnung über Aneignung zur teilweisen Übernahme von westlichen Modellen, die mit Modernität und Fortschritt gleichgesetzt wurden. Als Vorbilder dienten häufig westliche Protagonisten jener Zeit aus militärischen, wirtschaftlichen und politischen Bereichen.

Nach der Machtübernahme 1928 versuchte Chiang-Kai-shek, die Aufbauprogramme aus den „Drei Prinzipien des Volkes“ von Landesvater Sun Yat-sen auf das Bildungswesen zu übertragen. Ziele waren „Fortbestand der Nation“, „Unterstützung des gesellschaftlichen Lebens“ und „Unterstützung der Volkswirtschaft und des allgemeinen Wohlstands“. Die Bildungskonzepte verbanden unterschiedliche, z.T. widersprüchliche Elemente, so wurden unter Einfluss des Nationalismus in den 
1930er-Jahren auch feudalistische Elemente, wie Loyalität gegenüber Herrscher und Vaterland, wieder hinzugefügt, um die Staatsmacht zu stärken. Gleichzeitig begann die Gestaltung von differenzierenden Lehrgängen und Bildungszweigen, um den Arbeitskräftemangel in unterschiedlichen industriellen Sektoren zu beheben, die Verbesserung der Landwirtschaftsproduktion voranzutreiben und die Arbeitslosigkeit unter Jugendlichen und die dadurch entstehenden sozialen Unruhen zu unterbinden. Die Gestaltung des Bildungswesens wurde also nicht isoliert betrachtet, sondern mit der Entwicklung von Wirtschaft, Arbeitsmarkt und Gesellschaft koordiniert.

Nach ihrem Rückzug auf Taiwan setzten die Kuomintang ihre Konkurrenz mit dem kommunistischen Regime auf dem chinesischen Festland um den Alleinvertretungsanspruch für ganz China und die legitime Repräsentanz der chinesischen Kultur fort und suchten den Unterschied zum KPCh-Regime deutlich zu machen, indem sie sich als Bewahrer der konfuzianischen Werte und Traditionen empfahlen. Schritt für Schritt wurde das Modell des westlichen, staatlich kontrollierten Bildungssystems nach Taiwan importiert und umgesetzt, die Richtlinien lauteten „Erziehung mit Feder und Waffe“, „Bildung des Volksgeistes“ und „Produktions- und Arbeitserziehung“. Damit schlossen die Bildungsprogramme an die Regierungszeit in den 1930er- und 40er-Jahren an.

Doch zunächst war das KMT-Regime bestrebt, die Bevölkerung zu kampfwilligen und loyalen Streitkräften im Bürgerkrieg mit den chinesischen Kommunisten zu erziehen. Um die Kräfte für die Rückeroberung des chinesischen Festlands und den Kampf gegen den Kommunismus zu bündeln und loyale Parteigänger auszubilden, wurden Bildungseinrichtungen als politische Apparate für die Verbreitung der Staatsideologie sowie zur Überwachung der Jugendlichen genutzt. Das autoritäre 
Erziehungssystem wurde nicht nur durch die Bildungsbehörde kontrolliert, sondern auch durch Militärbehörden und Nachrichtendienst, so wurden militärische Instrukteure wie die Mitglieder des „China Youth Corps“ in den Bildungseinrichtungen eingesetzt, und Lehrinhalte, Schulverwaltung, die Ausbildung der Lehrkräfte, die Schaffung von Bildungsangeboten etc. standen unter staatlicher Aufsicht.

Nach der Niederlage im Bürgerkrieg bemühte sich das KMT-Regime vor allem darum, frei einsetzbare Arbeitskräfte auszubilden, um seine Wirtschaft zu stärken. Und da während des Kalten Krieges Taiwan als Frontabschnitt gegen den Kommunismus galt und von den USA in das Weltsystem unter US-amerikanischer Führung integriert wurde, erhielt das Land dank US-Entwicklungshilfe massive finanzielle und technische Förderungen zum Aufbau der naturwissenschaftlichen und technischen Bildung und Berufsausbildung, was vor dem Hintergrund der militärischen Konkurrenz zwischen den USA und der Sowjetunion, besonders nach dem Sputnik-Schock, zu sehen ist. Taiwan rutschte damit nicht nur in ein militärisches und wirtschaftliches, sondern auch ein bildungspolitisches Abhängigkeitsverhältnis zu den USA, denn pädagogische Theorien sowie Lehrpläne und -mittel wurden direkt aus den USA übernommen. Darüber hinaus wirkten internationale Organisationen wie UNESCO und die Weltbank durch Beratung und Finanzierung bei der Gestaltung des Bildungswesens mit. Der Westen hatte Vertrauen in die gemäß den „Drei Prinzipien des Volkes“ aufgebauten Bildungsprogramme des KMT-Regimes, welche eine enge Verbindung zwischen schulischer Ausbildung, wirtschaftlichen Leistungen und sozialer Stabilität vorsahen. Obwohl Taiwan zu Beginn der Nachkriegszeit noch eine bäuerliche Gesellschaft war, wurde nun eine ähnliche Bildungspolitik mitsamt Schulpflicht wie in den Industrieländern angestrebt. 
Die in den 1970er- und 1980er-Jahren vorgenommene Erweiterung der Bildungsangebote sollte vorderhand der Stärkung der internationalen wirtschaftlichen Wettbewerbsfähigkeit und der Verknüpfung mit dem Weltwirtschaftssystem dienen. Dazu sollten nun Facharbeiter, Techniker, Ingenieure, Kaufleute usw. im Zuge des staatlichen Aufbauprogramms sowie für private Unternehmen - arbeitskraftintensive Hochtechnologie und Export - ausgebildet werden. Um globalen Anforderungen zu genügen, wurden eine Reihe von „Plänen zur Ausbildung von Arbeitskräften“ nach den Konzepten „Human Ressource“ und „Funktionelle Differenzierung“ erstellt und mit der Gestaltung von differenzierten Ausbildungswegen für Facharbeitskräfte - von einfachen Facharbeitern bis hin zu professionellen Technikern und wissenschaftlichen Forschungskräften - durchgesetzt. So sollte das Bildungsideal vom konfuzianischen Gelehrten hin zur Ausbildung von Fachleuten umgestellt werden und naturwissenschaftliche und technische Erziehung immer mehr Gewicht und Wertschätzung gewinnen.

Doch die Mehrheit der Bevölkerung folgte in ihrer Einstellung zu Erziehung und Schulbesuch noch immer der konfuzianischen Tradition. Sie glaubte daran, dass ihren Kindern allein durch das Studium konfuzianischer Lektüre bzw. eine literarische Ausbildung Aufstiegsmöglichkeiten als Beamte oder Führungspersonen offen standen. Facharbeiter galten in der traditionellen Gesellschaft hingegen als Unterschicht. Hinzu kam, dass die überwiegend aus US-amerikanischen Lehrbüchern übernommenen Lehrinhalte der naturwissenschaftlichen und mathematischen Fächer für die meisten Schüler schwierig zu erlernen waren. Bereits in den 1950er-Jahren hatte Chiang Kai-shek den allein auf sozialen Aufstieg ausgerichteten, „blinden Bildungseifer“ der taiwanesischen Bevölkerung als rücksichtslos gegenüber dem Staatsaufbau bezeichnet. Auch später wurde dies von KMT-Politikern kritisiert. Durch die Einführung eines 
Aufnahmeprüfungssystems, mit dem das Regime mittels moderner Prüfungstechnik die Aufnahme neuer Schüler und Studenten zu steuern suchte, wurde eben dieser „blinde Bildungseifer“ indes befördert, denn ähnlich dem kaiserlichen Beamtenprüfungssystem ermöglichte es sozialen Aufstieg durch Bildung und individuelle Leistung. Darüber hinaus diente das System der politischen und erzieherischen Kontrolle, weil es dem KMT-Regime einen Mechanismus lieferte, das von ihnen diktierte „,ideale Menschenbild“ und die Staatsideologie in die Köpfe der jungen Bevölkerung einzupfropfen. Denn nur diejenigen Schüler, die der Staatsideologie folgten und die vom Staat diktierten Lehrinhalte beherrschten, waren erfolgreich und bekamen einen Bildungsplatz in der höheren Schule bzw. der Universität zugewiesen.

Im Fall der Modernisierung des Bildungswesens in Taiwan zeigt, dass es eine dauernde Auseinandersetzung zwischen den fremden und traditonellen Erziehungskonzepte war, dadurch einen $\mathrm{zu}$ eigener Gesellschaft angepassenden Weg zur Modernisierung zu finden, zugleich die nationale Identität zu bewahren. 


\section{Literaturverzeichnis}

\section{Quellen und Dokumente:}

Chiang Kai-shek 1934: Die zentralen Regeln der „Bewegung Neues Leben“ (新生活 運動之中心主旨) in: Propagate Commission in Central Committee of the KMT (Hrsg.) 中國國民黨中央執行委員會宣傳委員會 1935: Sammelband: Reden während der „Bewegung Neues Leben“. 新生活運動言論集. Nan-chang. 南昌. Bd. 1. S.21.

„Historic Commission in Central Committee of the KMT“ (Hrsg.) 中國國民黨中央 委員會黨史委員會主編. 1984: Sammelbände: Gedanken und Reden Chiang Kai-sheks. 先總統 蔣公思想言論總集. Taipei.

--1950: Rede an die Bevölkerung auf dem chinesischen Festland wegen des Abzugs der Truppen von Chou Shan und Hai Nan. 為撤退舟山、海南國軍告大陸同 胞書. Bd. 32. S.264-266.

--1951 (a): „Die Zeit überprüft die Jugend, die Jugend macht die Geschichte“ mit Erklärung über „Ssu-wei pa-te“ als wesentliche Waffe im Kampf gegen die Kommunisten und Russland. 時代考驗青年青年創造時代 並說明四維八德 為反共抗俄鬥爭中的主要武器. Bd. 24. S.197-206.

--1951 (b) : „Das Verhältnis zwischen der Erziehung und Revolution sowie dem Aufbau des Landes“،- zugleich Erklärung über die Bedeutung der Demokratie und Wissenschaft. 教育與革命建國的關係——並說明什麼是民主與科學. Bd. 24. S.207-213.

--1951 (c): Die Rekonstruktion der Bildung und die Änderung der Mentalität des Volkes. 改造教育與變化氣質. Bd. 24. S.264-274.

--1952 (a): Das Land braucht die revolutionäre Jugend, die Jugend braucht die 
revolutionäre Erziehung“، 國家需要革命青年 青年需要革命教育. Bd. 25 . S.98-103.

--1952 (b) : Rede zur Gründung des „China Youth Corps“. 中國青年反共救國團成 立大會講詞. Bd.25. S.173-176.

--1952 (c): Rede zum Fest des Jugendtages an alle Jugendlichen im Jahr 41 ROC 中華 民國四十一年青年節告全國青年書. Bd. 33. S. 11-14.

--1952 (d): Übersicht über die Schriften des Landesvaters. 國父遺教概要. Anhang II:

Das Wesen der „Drei Prinzipien des Volkes“ - Ethik, Demokratie, Wissenschaft-。附錄二 三民主義的本質 - 倫理、民主、科學 -. Bd. III. S.157-181.

--1953: Zwei Aufsätze über Yu und Le zur Ergänzung des Prinzips des Volkswohls. 民生主義育樂兩篇補述. Bd. III. S.191-260.

--1966: „Rede zum einhundertersten Geburtstag von Sun Yat-sen und die Gründung der Halle der chinesischen Kultur im Erinnerungshaus Chung-shan“.中山樓 文化堂落成紀念文國父一百零一年誕辰紀念文. Bd.35. S.270-272.

--1968: „Punkte zur Beachtung bei der Erneuerung der Erziehung“. 對革新教育注意 事項之指示. Bd.37. S.425-433.

Sun Yat-sen 孫中山 1927: Die "Drei Prinzipien des Volkes“. In: Hsu Wen-shan (Hrsg.) 徐文珊編 1969: Sammelband: Die „Drei Prinzipien des Volkes“. 三民主義 總集. Taipei.

--1894: Brief über Reformvorschläge an den Hochbeamten Li Hung-chang. 上李鴻 章書. In: Kommission für die Ausgabe der Sammelbände des Landesvaters (Hrsg.) 國父全集編輯委員會編 1973：Sammelband des Landsvaters. 國父全集. Bd. I. S.83-95. Taipei.

--1923: „Rede über das Studium zur Verbesserung des Gemeinwohls, nicht aber zur Förderung des Hochbeamtentums“. 要立志做大事,不要做大官. Gehalten am 
21. Dezember 1923 an der Lin Nan Universität in Kanton. 民國十二年 十二月二十一日 於廣州嶺南大學 In: Kommission für die Ausgabe der Sammelbände des Landesvaters (Hrsg.) 國父全集編輯委員會1973: Sammelband des Landsvaters. 國父全集. Bd. II. S.8. Taipei

Wirtschafsministerium (Hrsg.):

--1971: Der erste Vierjahresplan zum wirtschaftlichen Aufbau ROC. 中華民國第一 期台灣經濟建設四年計畫.

--1971: Der zweite Vierjahresplan zum wirtschaftlichen Aufbau ROC 中華民國第二 期台灣經濟建設四年計畫

--1971: Der dritte Vierjahresplan zum wirtschaftlichen Aufbau ROC (1961-1964). 中 華民國第三期台灣經濟建設四年計畫.

--1971: Der vierte Vierjahresplan zum wirtschaftlichen Aufbau ROC (1965-1968). 中 華民國第四期台灣經濟建設四年計畫.

--1972: Der fünfte Vierjahresplan zum wirtschaftlichen Aufbau ROC (1969- 1972). 中華民國第五期台灣經濟建設四年計畫.

--1973: Der sechste Vierjahresplan zum wirtschaftlichen Aufbau ROC (1973- 1976). 中華民國第六期台灣經濟建設四年計畫.

--1976: Der Sechsjahresplan zum wirtschaftlichen Aufbau ROC (1976-1981). 中華民 國台灣六年經濟建設計畫.

„Council for International Economic Cooperation und Development“ under Exkusiv Yuan“(Hrsg.) 行政院國際經濟合作發展委員會編:

--1964: Kritik an dem Erziehungsprojekt unter der US-Entwicklungshilfe. 美援教育 計劃檢討.

--1966 : Bericht über die „Ausbildung von Arbeitskräften“ in Taiwan. 台灣人力資源 專題報告.

--1968: Der „revidierte Plan zur Ausbildung von Arbeitskräften“. 人力發展計畫修 
正草案.

--1970: „Der dritte Plan zur Ausbildung von Arbeitskräften“. 第三期人力發展計劃 草案.

--1970: Bericht über die Durchführungsergebnisse des „Plans zur Ausbildung von Arbeitskräften“. 人力發展計畫工作成果檢討報告.

--1970: Übersicht über die Darlehensmaßnahmen der Weltbank. 世銀貸款簡介. In: Material für die dritte Konferenz über Human Capital der Gruppe für Erziehungsentwicklung. 第三屆全國人力研討會參考資料 教育發展組.

--1972: „Der vierte Plan zur Ausbildung von Arbeitskräften“(1971-1981) 第四期人 力發展計劃草案.

„Council for Economic Planning and Development ROC“ under Exkusiv Yuan“(Hrsg.). 行政院經濟建設委員會編:

--1982: Bericht über das Studium der Planung zur Ausbildung von Arbeitskräften. Bd. II. 人力規劃研究報告 $($ 二 $)$

--1986: Mittel- und langfristiger Plan zur Ausbildung von Arbeitskräften zum wirtschaftlichen Aufbau ROC. (1986-1990) 中華民國台灣經濟建設人力發 展部門中長期計畫，民國七十五年至八十九年.

-- Taiwan Statistical Data Book 1978, 1995, 1996, 2007.

Statistikbehörde der Verwaltungsbehörden des Gouvernements der Provinz Taiwan. (Hrsg.) 台灣省行政長官公署統計室編. 1946: Der Überblick der 51 Jahre Statistik in der Provinz Taiwan. 台灣省五十一年統計提要.

Bildungsbehörde der Provinz Taiwan. (Hrsg.) 台灣省政府教育廳編 1951: Untersuchungsbericht über die Volksschule und Mittelschule im Jahr 1951 in der Provinz Taiwan. 台灣省五十年國民教育及中等教育調查報告書.

Bildungsbehörde der Verwaltungsbehörden des Gouvernements der Provinz Taiwan. (Hrsg.) 台灣省行政長官公署教育處編 1955: Zehn Jahre Erziehung in 
Taiwan. 十年來的台灣教育.

Directorate General of Budget, Accounting and statistics in Province Taiwan (Hrsg.) 臺灣省主計處編 1959: Überblick der Statistik der Provinz Taiwan. 台灣省 統計要覽. Bd. 14.

Directorate General of Budget, Accounting and Statistics, Executive Yuan 行政院主 計處 2009: Yearbook of manpower survey statistics Taiwan area, Republic of China. 中華民國台灣地區人力資源調查統計年報.

Abteilung für Bevölkerungsstatistik unter dem Innenministerium (Hrsg.) 內政部人 政策資料委員會編 2006: Sammelband der Bevölkerungsstatistik. 人口政策 資料彙集.

Directorate General of Budget, Accounting and Statistics under Executive Yuan (Hrsg.) 行政院主計處編: Jahrbuch der Statistik ROC. 中華民國統計年鑑. 1987,1997, 2007.

Bildungsministerium (Hrsg.):

--1957: Das dritte Jahrbuch der Bildung ROC. 第三次中華民國教育年鑑.

--1974: Das vierte Jahrbuch der Bildung ROC. 第四次中華民國教育年鑑.

--1984: Das fünfte Jahrbuch der Bildung ROC. 第五次中華民國教育年鑑.

--Educational Statistics ROC. 中華民國教育統計：1970，1971，1972，1997，1998， 2002, 2008.

--,Stanford Research Institute“ in California, USA．美國加州司丹佛研究所 1962: Education and Development. The Role of Educational Planning in the Economic Development of the Republic of China. 教育與發展. 中華民國經 濟發展過程中教育計劃之任務. Chinesische Ausgabe der Zentralen Kommission für Zusammenarbeit zwischen schulischer und außerschulischer Ausbildung unter dem Bildungsministerium. 中文版 教育部中央建教合作 委員會. 
--UNESCO und Zentrale Kommission für Zusammenarbeit zwischen schulischer und außerschulischer Ausbildung unter dem Bildungsministerium. (Hrsg.) 教育部 中央建教合作委員會編 1964: „Bildungsplan der ROC - Langfristiger Bildungsplan (1964-1982)““中華民國教育計畫-長期教育計畫 民國 53-71 年.

The National Institute of Educational Resources and Research (Hrsg.) 國立教育資料 館編:

--1957: Bericht über die Durchführung der Erziehungsprojekte unter der US-Entwicklungshilfe. 美援有關教育計畫實施報告.

--1959: Sieben Jahre Zusammenarbeit dem auf dem Bildungssektor zwischen den USA und der Republik China 。七年來的中美教育合作.

Center for Educational Research NTNU 1994: Studie über die Reform der Senior High School unseres Landes für das Jahr 2000. 我國公元兩千年高級中等教 育改革方向之研究. In: Bulletin of the National Institute of Education Materials

„Sino-American Joint Commission on Rural Reconstruction“ (Hrsg.) 中國農村復 興聯合委員會編 1951: Arbeitsberichte der „Sino-American Joint Commission on Rural Reconstruction“. 中國農村復興聯合委員會工作報告. 1950-1951. Bd.2

\section{Literatur in chinesischer Sprache:}

An Hou-wei 安後暐 1998: Die Einflüsse der US-Entwicklungshilfe auf die Berufausbildung in Taiwan 1940-1965. 美援對台灣職業教育的影響 (民國 39-54 年). Magisterarbeit am Institut für Geschichte an der „National Taiwan Normal University“. 國立台灣師範大學歷史研究所碩士論文.

Andrade, Tonio 歐陽泰 2007: How Taiwan became Chinese? 福爾摩沙如變成台灣 
府? Chinesische Aufgabe. Taipei.

Bai Hsi-liang 白新良 1995: Die Entwicklungsgeschichte der ”Akademien” (Shu-yuen) im alten China. 中國古代書院發展史. Tien-chin.

Die Bildungskommission des Völkerbunds 國聯教育考察團 1963: The Reorganisation of Education in China 1932. 中國教育之改進. Chinesische Ausgabe. Taipei.

Campbell, Willim 2001: Formosa under the Dutch: described from contemporary records, with explanatory notes and a bibliography of the island. 荷據下的福 爾摩沙. Taipei.

Cha Shih-chieh 查時傑 1984: Die historischen Hintergründe für die Entstehung der anti-christlichen Bewegung in den 1920er Jahren (1922-1927). 民國十年代非 基督教運動產生的時代背景 (1922-1927) In: Sammelband: Debatten über Geschichte und Kultur der Republik China. 中華民國歷史與文化討論集. Bd.3. Die kulturelle und gedankliche Geschichte. 文化思想史. Taipei. S. 148-396.

Chaffee, John W. 賈志揚 1995: Die kaiserliche Aufnahmeprüfung in der SongDynastie. 宋代科舉. Taipei.

Chan Sheng-ju 詹勝如 2000: Studie über die Erziehungspolitik der Weltbank. 世界 銀行教育研究之研究. Magisterarbeit beim Institut für vergleichende Pädagogik an der „National Chi Nan University”. 國立暨南大學比較教育研 究所碩士論文.

Chan Yoa-chi 詹曜齊 2005: Polemik über Modernisierung und die Bewegung der Modernisierung auf Taiwan. 台灣的現代化論戰與現代化運動. Magisterarbeit beim Institut für die Gesellschaftsentwicklung an der „Shi Hsin University”. 世新大學社會發展研究所碩士論文.

Chan Wei 詹瑋 1992: Wu Chih-hui und die Bewegung der Hochchinesischen Sprache. 
吳稚暉與國語運動. Taipei.

Chang An-jan 張安然 1987:Erziehungsgedanken der “Drei Prinzipien des Volkes”三 民主義的教育思想. Taipei.

Chang Chi-yun. 張其昀 1956: Die Ergebnisse der Durchführung von zwei wichtigen Maßnahmen aus dem Bildungsministerium. 教育部兩大方案實施情況. In: Erziehung und Kultur 教育與文化 Vol.13 (10), 1956, S. 35.

--1982 (a) : Memoiren über meine Amtszeit im Bildungsministerium (1954-1958). 景 福門回憶錄. Hua Gang Gesellschaft an der „Chinese Culture University“(Hrsg.) 中國文化大學華岡學會編 1982: Das Leben und die Gedanken von Dr. Chang Chi-yun. 張其昀博士的生活和思想. Bd.I 上冊 S. 209-282.

--1982 (b) : Der Prozess des Wiederaufbaus der „National Cheng Chi University”. 政 治大學在台復校之經過. Hua Gang Gesellschaft an der „Chinese Culture University“(Hrsg.) 中國文化大學華岡學會編 1982: Das Leben und die Gedanken von Dr. Chang Chi-yun. 張其昀博士的生活和思想. Bd.I S. 464-467.

Chang Chih-tung 張之洞 1971 : Die kaiserliche Regelung für die Gestaltung des Bildungswesens. 奏定學堂章程. Taipei.

Chang Ching-hsi und Wu Hui-ling 張清溪、吳惠林 1996 :Soll die Bildung dem Zweck der Wirtschaftsentwicklung dienen? 教育應以經濟發展為目的? Taipei.

Chang Chung-tung 張忠棟 1990: Hu Shih, Wang Shih-chian, Lei Chen, Yin Hai-guang. 胡適, 雷震, 殷海光. Taipei.

Chang Chung-wei 張仲瑋 2005: Kleine Vorstellung über den emaligen Präsident der Universität Tsing Hua, Herr Hsu Hsien-hsiu. 淺談前校長徐賢修先生. Hsing-chu.

Chang Li 張力 1986: Die technische Zusammenarbeit zwischen China und dem 
Völkerbund in den 1930er Jahren. 一九三零年代中國與國聯的技術合作. In: Bulletin of the Institute of Modern History Academia Sinica. 中央研究院近代 史研究所集刊. Vol. 15 (2) Dec. 1986 S. 281-314.

Chang Hai-pung und Tao Wen-chao 張海鵬 陶文釗 2010 : Kürze Geschichte zu Taiwan. 台灣簡史. Nan King.

Chang Po-chung 張柏仲 1996: The Social-psychological Analysis of Taiwan's Mainlanders. 外省族群的社會心理分析:族群觀點、我群認同與社會距離. Magisterarbeit beim Institut für Politikwissenschaft an der „Tung Wu University”. 東吳大學政治研究所碩士論文.

Chang Po-yu (Hrsg.) 張博宇編: Dokumente der Bewegung zur Verbreitung der Nationalsprache im Gebiet Taiwan. 台灣地區國語運動史料. Taipei.

Chang Shih-Hsien 張世賢 2009: Die Politik zu Taiwan während Ende ChingKaiserreich 1874-1895. 晚清治台政策 1874-1895. Taipei.

Chang Shu-lun 張樹倫 1998: Der soziale Wandel und die Konstruktion der Kultur auf Taiwan. 臺灣地區社會變遷與文化建設. Taipei.

Chang Shu-ya 張淑雅 1990: Studie zum Wandel der US-Politik bezüglich Taiwan im Zeitraum von 12.1950 bis 05.1951. 美國對台政策轉變的考察.

Chang Ya-chun 張亞群 2005: Die Abschaffung des kaiserlichen Beamtenprüfungssystems und die Umwandlung der Hochschulbildung im modernen China. 科 舉革廢與近代中國高等教育的轉型. Shang Hai.

Chang Yuh-fa 張玉法 1977: Die Geschichte des modernen China. 中國現代史. Taipei.

--1998: Skizze zur Geschichte der Republik China. 中國民國史稿. Taipei.

Chao Chi-chang 趙既昌 1985: Die Verwendung der US-Entwicklungshilfe. 美援的運 用. Taipei.

Chao Ena 趙綺娜 2001: U.S. Educational and Cultural Exchange Programs in Taiwan 
(1951-1970). 美國政府在台灣的教育與文化交流活動 (一九五一至一九七).

In: A Journal of European and American Studies. 歐美研究. Vol. 31 Nr. 1, March 2001, S. 79-127.

Chen Ban-chen 1988. 陳邦禎：Das Studium über akademische Gedanken von Ku Tin-ling. 顧亭林先生學術思想研究. Dissertation beim Institut für Chinesische Literatur an der „Chinese Culture University“. 中國文化大學中國 文學研究所博士論文. Taipei.

Chen Bo-chang 陳伯璋 1991: Die Illusion der Volkserziehung. 國民教育的迷思. In: Yang Ying (Hrsg.) 楊瑩編 1991: Pädagogik in der sich wandelnden Gesellschaft. 轉型社會中的教育. Taipei. S. 75-84.

Chen Chao-nan, Sun Te-hsiung und Li Tung-ming 陳肇男、孫得雄、李棟明 2003: Das Wunder des Bevölkerungswandels in Taiwan. 台灣的人口奇蹟. Taipei.

Chen Cheng-jan 陳正然 1985: Die Kulturbewegung der Intellektuellen in den 1960er Jahren auf Taiwan am Beispiel der Zeitschrift ,Wen-hsing“. 台灣五零年代知 識分子的文化運動以「文星」為例. Magisterarbeit beim Institut für Soziologie an der „National Taiwan University”. 台大社會學研究所碩士論文.

Chen Chi-ming 陳啟民 2000: Analyse der Maßnahmen des KMT Regime für Festlandchinesen auf Taiwan. 國民黨對外省族群的統治分析. Magisterarbeit beim Institut für Politikwissenschaft an der „National Chen Chi University”. 國 立政治大學政治學研究所碩士論文.

Chen Chun-hua 陳俊華 2008: Die Studie über die neue Erziehung am Ende der Ching-Dynastie 清末新式教育之研究. Ping-tong.

Chen Fang-ping und Bi Chen 程方平和畢誠 1996: Die Bildungsgeschichte Chinas. 中國教育史. Taipei.

Chen Hsiao-chung 陳小沖 2005: Die fünfzigjährige Geschichte der japansichen Kolonie auf Taiwan. 日本殖民統治臺灣五十年史. Peking. 
Chen Jung-chieh 陳榮捷 1990: Chu Hsi. 朱喜. Taipei.

Chen Kao-xiang 鄭學稼 1979: Die Führung der Bewegung des Vierten Mai Zeitschrift „Neuen Jugend“. 主導五四時代的新青年雜誌. In: Chou Yang-shan 周陽山 (Hrsg.) 1979: Der Vierte Mai und China. 五四與中國. Taipei. S. 513-525.

Chen Li-fu 陳立夫 1973: Memoiren über die Verwaltung des Bildungssystems während des sino-japanischen Krieges. 戰時教育行政回憶錄. Taipei.

Chen Mei-ju 陳美如 1996: Studie über die Sprachpolitik nach der Rückgabe Taiwans an China. 台灣光復後語言教育政策之研究. Magisterarbeit beim Institut für Pädagogik an der „National Taiwan Normal University“. 國立台灣師範大學 教育研究所碩士論文.

Chen Ta-hwa 鄭大華 1997 ：Biographie von Chang chün-mai. 張君勱傳. Taipei.

Chen Teng-yun 鄭登雲 1994: Die Bildungsgeschichte des modernen China. 中國近 代教育史. Shang Hai.

Chen Tien-hsi, Yu Huan-mo und Chang Pi-chi 鎮天錫, 余煥模, 張丕繼 1983: Entstehung und Durchführung der Politik zur „Ausbildung der Arbeitskräfte“. 人力政策的形成與實施. Taipei.

Cheng Wan-chi 鄭婉琪 2002: Politische Kontrolle, Regulierung der Bildungsangebote und : eine historsch-soziologische Analyse. 政治控制、教育管制與 升學主義:歷史社會學的詮釋.

Chen Yao-hong 陳耀宏 1992: Wandlungsprozess des „China Youth Corps“ Freizeitveranstaltung.中國青年反共救國團全國性動態青年活動之歷 史變遷. Magisterarbeit beim Institut für Sportwissenschaft an der „National Taiwan Normal University“. 國立台灣師範大學體育研究所碩士論文.

Chen Yueh-ping 陳月萍 2003: Erziehung der Überseechinesen und Antikommuni- 
smus im Zug der US-Entwicklungshilfe (1950-1965). 美援的華僑教育與反共 活動 (1950-1965). Taipei.

Cheng Tzu 鄭梓 1994: Übernahme und Wiederaufbau Taiwans nach dem Zweiten Weltkrieg. 戰後台灣的接收與重建. Taipei.

Chiang Pei-ching 江佩津 1997: Landwirtschaftserziehung in Taiwan unter der japanischen Besatzung (1895-1945). 日治時代台灣的農業教育(1895-1945). Magisterarbeit beim Institut für Geschichte an der „National Central University“. 國立中央大學歷史研究所碩士論文.

Chien Man-chien und Chin Lin-hsiang (Hrsg.) 錢曼倩和金林祥主 1996: Studie zum Vergleich der modernen Bildungssysteme.中國近代學制比較研究. Kanton.

Chien Mu 錢穆 1977 : Die politischen Erfolge und Misserfolge in den Dynastien Chinas. 中國歷代政治得失. Taipei.

Chin Hsiao-i (Hrsg.) 秦孝儀編 1981: Geschichte der Kulturentwicklung der Republik China. 中華民國文化發展史. Bd. 4. Taipei.

Chin Sui-ling 秦穗齡 2004: Pfadfinder und Ausbildung der chinesischen modernen Jugendlichen. (1911-1949) 童子軍與現代中國的青少年訓練（1911-1949). Magisterarbeit beim Institut für Geschichte an der „National Taiwan Normal University“. 國立台灣師範大學歷史研究所碩士論文.

„Chinese Association for the Advancement of Nature Science“ (Hrsg.) 中國自然科 學促進會編 1956: Probleme der Naturwissenschaftserziehung und ausrüstungen in den High Schools. 中等學校理科教育和設備問題. In: Naturwissenschaftserziehung. 科學教育. 2 (2). S. 55-56.

--1960: Untersuchungsbericht über die Naturwissenschaftserziehung in den High Schools in der Provinz Taiwan. 台灣省中等學校科學教育調查報告. In: Naturwissenschaftserziehung. 科學教育. 6 (1). S. 59-64.

Chiu Ai-ling 邱愛玲 1998: A Study of Changes of the Joint College Entrance 
Examination Policies in Taiwan from 1954 to 1997. 我國大學聯招政策變遷 之研究 1954-1997. Dissertation beim Institut für Pädagogik an der „National Taiwan Normal University“. 國立台灣師範大學教育研究所博士論文.

Chiu Hei-yuen 翟海源 2003: Das Streben nach höherer Bildung. Eine Analyse des taiwanesischen Bildungswesens und der Werte der Bildung seit der ChingDynastie. 追求高教育成就 - 清代以來臺灣教育制度與價值的分析. In: Taiwan Journal of Sociology of Education. 臺灣教育社會學研究. No.3 (2): $1-39$.

Chiu Hsiu-hsiang 邱秀香 2005: Von Realerziehung zur Berufsausbildung: Eine Studie zum Verhältnis zwischen sozialer Entwicklung des modernen Chinas und Ausbau der Erziehung (1904-1922). 〈從實業教育到職業教育:論近代中國社 會發展與教育推行之關係 (1904-1922)〉 In: Fu Jen Historical Journal.《輔 仁歷史學報》, 16 vol. 2005.7. S. 130-132.

Chou Chi-wen 周期文 1987: Erziehung durch Experimente: Rückblick und Vorausschau . 實驗教育的回顧與前瞻. In: Erziehung in Taiwan. 台灣教育. Vol. 433, 1987, S. 4-8.

Chou Tan-huei 周談輝 1985: Entwicklungsgeschichte der beruflichen Ausbildung in China. 中國職業教育發展史. Taipei.

Chou Yu-wen 周愚文 2001: Grundriss der chinesischen Bildungsgeschichte. 中國教 育史綱. Taipei.

Chow Tse-tsung 周策縱 1989: The May Fourth Movement. Intellectual Revolution in Modern China. 五四運動史. Chinesische Ausgabe. Taipei.

Chu Ching-I, Tai Hua und Chen Chih-chia 朱敬一、戴華、陳致嘉 1995: Die Rolle des Staates in der Erziehung. 國家在教育中的角色. Taipei.

Chu Je-ying 祝若穎 2011: Die Entwicklung der modernen Erziehungsgedaken des Westens und ihre Einflüsse auf die Didaktik in den öffentlichen Schulen unter 
der japanischen Besatzung (1895-1945). 日治時期西方近代教育思想之發展 與對公學校教學法之影響 (1895-1945). Dissertation beim Institut für Pädagogik an der „National Taiwan Normal University“. 國立台灣師範大學 教育研究所博士論文.

Chu Hsi 朱喜 2007: Vier Bücher unter den Kommentaren Chu Hsi’s.四書集注 Taipei.

Chu Pei-chi 朱㛂琪 2000: Die Wiege der taiwanesischen Elite unter der japanischen Besatzung am Beispiel der Taichung Mittelschule. 台籍菁英的搖籃：台中一 中. Magisterarbeit beim Institut für Geschichte an der „National Tsing Hua University”. 國立台灣清華大學歷史研究所碩士論文.

Chung Ching-han 鍾清漢 1988: Studie über Ausbildung und Wirtschaftsentwicklung. 教育與經濟發展之研究. Taipei.

Chung Han-shu 鍾瀚樞 2004: Die Erziehungsprojekte der US-Entwicklungshilfe auf Taiwan in den 1950er Jahren. 1950 年代台灣的美援教育計畫. Magisterarbeit beim Institut für Geschichte an der „Tang Hai University“. 東海大學歷史研究 所碩士論文.

Chyu Li-ho 翟立鶴 1984 (a): Die Zeitströmungen nationalistischer Erziehung am Ende der Ching-Dynastie und zu Beginn der Republik China. 清末民初民族主 義教育思潮. Taipei.

--1984 (b): The Educational Thoughts of Military-Training in Modern China. 清末民 初軍國民教育思潮. In: Journal of National Taiwan Normal University. 台灣 启大學報. Nr.29 S. 27-52.

Eckstein, Max A. und Noah, Harold J. 1996: Secondary School Examination: International Perspectives on Politicise and Practice. 邁向大學之路一各國考 試制度的理論與實際. Chinesische Ausgabe. Taipei.

Elman, Benjamin A. 艾爾曼 2010: Kulturelle Geschichte der modernen 
Wissenschaft. 中國近代科学的文化史. Shang-hai.

Fang Chun-yu 方俊育 2000: Technology or Politics--The Development of Industrial Vocational Education in Taiwan (1945-1986). 技術或政治-台灣戰後工業職 業教育發展史 (1945-1986). Magisterarbeit beim Institut für Geschichte an der „National Tsing Hua University”. 國立清華大學歷史研究所碩士論文.

Fu Li-yuh 傅麗玉 2006 (a): Secondary Science Education in Taiwan under US AID (1951-1965): Initiation and Implementation. 美援時期台灣中等教育科學發 展. In: Chinese Journal of Science Education. 科學教育學刊. 2006, 14 (3), S. 333-380.

--2006 (b): A Chronology of the Initiation and Implementation of the Secondary Science Education Project under the U.S. Aid in Taiwan (1951-1965). 美援時 期台灣中等科學教育計畫之形成與實施年表 (1951-1965). In: Chinese Journal of Science Education. 科學教育學刊. 2006. 14 (4), S. 447-465.

Fan Shih-tuo 方師鐸 1965: Die Geschichte der Bewegung zur Verwendung der Nationalsprache seit den fünfzig Jahren. 五十年來中國國語運動. Taipei.

Gu Schang-sheng 顧長聲 2004: Missionaries and Modern China. 傳教士與近代中 國. Shang Hai.

Ku Po-yen 顧柏岩 1958: Die fünf Stufen der Berufe in der Industrie. In: Taiwanesische Expedition der gewerblichen Ausbildung (Hrsg.) 1958: Bericht über die Untersuchung der taiwanesischen industriellen Berufe in ROC 1958. Taipei.

He Ching-chin 何清欽 1980: Die taiwanesische Erziehung zu Beginn der Rückgabe an China. 光復初期之台灣教育. Kaohsiung.

--1985: Studie über die Entstehung des neuen Bildungswesens im Jahr 1922. 民國十 一年新學制釀成過程之探討. In: Educational Review Forum. 教育學刊. Nr.5,1985, S. 41-93. 
He I-lin 何義麟 1986: Studie über die Politik der Japanisierung. 皇民化政策之研究. Die Bildungspolitik und Bewegung der Japanisierung am Ende der japanischen Besatzung von Taiwan. 日據時代末期日本人對台灣的教育政策與教化運動. Magisterarbeit beim Institut für Japanese Studies an der „Chinese Culture University”. 文化大學日本研究所碩士論文.

Hong Rui-chong 洪瑞重 1998: Übernahme und Fortsetzung der Erziehung in der Übergangszeit durch Verwaltungsbehörden des Gouvernements der Provinz Taiwan (1945-1947). 臺灣省行政長官公署時期教育的接收與推展 (1945-1947). Magisterarbeit beim Institut für Geschichte an der „National Taiwan Normal University“. 國立師範大學歷史研究所碩士論文.

Hsiao Kung-chuan 蕭公權 1982: Die Geschichte des politischen Denkens in China. Bd.1. 中國政治思想史 (上). Taipei.

Hsieh Li-fa 謝里法 1997: Die taiwanesische Kunstbewegung unter der japanischen Besatzung. 日據時代台灣美術運動史. Taipei.

Hsiung Ping-chen 熊秉真 1992: Guter Anfang: Erziehung der frühen Kindheit von Gelehrten des modernen China. 好的開始--中國近世士人子弟的幼年教育. In: Sammlung der Geschichte: Familie und Politik im modernen China. 近世家 族與政治比較歷史論文集. S. 201-238.

Hsu Fu-ming 許福明 1986: Reorganisation der Nationalpartei Chinas 1950-1952 und deren Einflüsse auf die politische Entwicklung der ROC. 中國國民黨的改造 1950-1952 兼論其對中華民國政治發展的影響. Taipei.

Hsu Kuang-tai 徐光台 2003: Begegnung zwischen Neo-Konfuzianismus und Jesuiten am Ende der Ming-Dynastie und zu Beginn der Ching-Dynastie am Beispiel der Aufklärungserfahrung von Hsiung Ming-yu. 明末清初理學與耶穌會教育傳 統的遭遇：以熊明遇的啟蒙為例. In: Chu Han-ming (Hrsg.) 朱漢民主編 2003: Die chinesischen „Shu-yuen“ 中國書院. Bd. 5. Chang Sha. S. 445-471. 
--2004: Reflections on the Introduction of Western Education into Late Ming China. 明末西方教育的傳入及歷史反思. In: Taiwan Journal of East Asian Studies. 臺灣東亞文明研究學刊. Bd.1 Heft 2, 2004, Taipei. S. 77-104.

Hsu Ming-hua 徐明華 1993: Die Academia Sinica und die Institutionalisierung der Forschung. 中央研究院與中國科學研究的制度化. In: Bulletin of the Institute of Modern History Academia Sinica. 中央研究院近代史研究所集刊. Vol.22. 1993. 06. S. 233-254.

Hsu Hsi-ching 許錫慶 2008: Der Prozess über die Entbindung der Taiwanesen von Chinesischer Identität am Beispiel der Situation der elementaren Bildung zu Beginn der japanischen Besatzung. 台灣漢人首度掙脫中國情結的心路歷 程一以日治初期在台灣時初等教育的概況為著作為探討。In: Sammelband von fünfter Tagung über Dokumenten des Gouvernement Taiwans. 2008. 第五 屆台灣總督府檔案學術研討會論文集. Taipei.

Hsu Nan-hao 徐南號 1984: Die Entstehung und Entwicklung des Bildungswesens in Taiwan. In: Gesellschaft für vergleichende Pädagogik (Hrsg.) 1984: Die Reformtendenz der weltlichen Bildungssysteme.

--1996: Die taiwanesische Bildungsgeschichte - Rückblick und Aussichten. 台灣教 育史之回顧與展望. In: Hsu Nan-hao (Hrsg.) 徐南號主編 1996: Die taiwanesische Bildungsgeschichte. 台灣教育史. Taipei. S. 211-236.

Hsu Pei-hsien 許佩賢 2001: Die Geburt der modernen Schule in Taiwan: Aufbau des elementaren Bildungssystems unter der japanischen Besatzungszeit (18951911). 臺灣近代學校的誕生：日本時代初等教育體系的成立 (1895-1911). Dissertation beim Institut für Geschichte an der "National Taiwan University". 國立臺灣大學歷史研究所博士論文.

Hsu Tzu 徐梓 2006: Die Aufgabe der traditionellen elementaren Erziehung - These von Chu-Hsi 傳統啟蒙教育的使命-朱喜的論說. In: Lee Thomas H.C. (Hrsg.) 
李弘祺編 2006: Tradition der Erziehung in China und Ostasien Bd. 1. - Die Erziehung und das kaiserliche Beamtenprüfungssystem in China. 中國與東亞 的教育傳統(一)：中國的教育與科舉. Taipei. S. 257-282.

Hsueh Hsiao-hua 薛曉華 1996 : Die Bildungsreform aus den Initiativen auf Taiwan: Eine Analyse aus Sicht des Staates und der Gesellschaft. 臺灣民間教育改革: 國家與社會的分析. Taipei.

Hsueh Hua-yuan 薛化元 1995: Untersuchung über die Einstellung zur Demokratie in der Zeitschrift „Free China“ - eine Studie über die gedankliche Geschichte in den 1950er Jahren auf Taiwan. 《自由中國》雜誌自由民主理念的考察 - 一 九五 0 年代台灣思想史研究. In: Studie of Taiwan History Academia Sinica. 台灣史研究. Bd. 2 (1), 1995, Taipei. S.1 27-160.

Hu Chang-chih 胡昌智 1987: Das Bildungswesen und die chinesischen Intellektuellen unter dem Einfluss des Historismus: Die gesellschaftliche Reaktion auf den Bericht über die „Reorganisation des chinesischen Bildungswesens“ aus dem Völkerbund und dessen Einfluss. 歷史主義的教育制度與中國知識份子:國聯 中國教育考察團報告書的社會思想及其影響. In: Journal of History of University Tung Hai. 東海大學歷史學報. Vol. 28.,Juni 1987, S. 37-50.

Hu Shih 胡適 1960: Biographie Ting Wen-chiang. Taipei.

--1961: What are the social changes necessary for the growth of science in our countries in the Far East? Speech delivered at SECRAID, Nov. 6, 1961 at JCRR Conference Room.

--1962 (a): Social changes and science. In: Free China Review, March, S. 39-41.

--1962 (b): „Social Changes Necessary for Science Development“. 發展科學所需要 的社會. In: Zeitschrift ,,Wen-hsing”. 文星雜誌. Heft 50. S. 5.

Hu Shih-ching 胡世慶 2009: Geschichte der chinesischen Kultur. 中國文化通史. Taipei. 
Huang Chi-wen 黃琦雯 2005: Studie über die Frauenerziehung in der SpätChingDynastie. 清末女學研究. Magisterarbeit beim Institut für Geschichte an der „Tam Kang University”. 淡江大學歷史研究所碩士論文.

Huang Chia-shu 黃嘉樹 1991: Die nationalistische Partei Chinas auf Taiwan. 國民黨 在台灣 (1945-1988). Hai Kou.

Huang Chu-li 黃茱莉 2005: A Reflection on the Practice of Baden-Powell's Scout Education Thought in the Republic of China. 貝登堡童軍教育思想在我國實 踐之省思. Magisterarbeit beim Institut für Pädagogik an der „National Tung Hua University“. 國立東華大學教育研究所碩士論文.

Huang Chun-chieh 黃俊傑 1992: “Confucianism in Postwar Taiwan” Proceedings of the National Science Souncil ROC, Part C. In: Humanities and Social Sciences No.2. Taipei.

--1996: Studie der fernöstlichen Kultur: Die Entwicklung der traditionellen Kultur. 東 亞文化的探索:傳統文化的發展. Taipei.

--2001: Confucianism and Modern Taiwan. 儒家與現代台灣. Peking.

Huang Chung 黃中 1980: Die Entwicklung der modernen Erziehung unseres Landes. 我國近代教育的發展. Taipei.

Huang Fu-ching 黃福慶 1975:Chinese Students in Japan during the late Ching Period 清末留日學生. In: Bulletin of the Institute of Modern History Academia Sinca. 中央研究院近代史研究所專刊. S. 147-187.

Huang Guang-liang 黃光亮 1976: Studie über das kaiserliche Beamtenprüfungssystem in der Ching-Dynastie. 清代科舉制度之研究. Taipei.

Huang Hsu-ten/Wang Jen-wu/Ling Hong-yu/ 黃旭田、林烘煜、王震武1996: Das Wesen der schulischen Organisation in Taiwan am Beispiel der Volkserziehung. 台灣教育組織的體質-以國民教育為中心的分析. Taipei.

Huang Kuo-feng 黃國峰 1987: Studie über die Sprachreform unter den chinesischen 
Kommunisten. 中共文字改革之研究. Magisterarbeit beim Institut für „Chinese Studies“a an der „Chinese Culture University”. 文化大學大陸問題研 究所碩士論文.

Huang Kuo-lu 黃國倫 2007: Simplified Chinese Characters Research. 簡化字研究. Magisterarbeit beim Institute für „Chinese and Chinese Literature“ an der „Hsuan Chuang University”. 玄奘大學中國與文學研究所碩士論文.

Huang Te-fu 黃德福 1992: Demokratische Fortschrittspartei und politische Demokratisierung auf Taiwan. 民主進步黨與台灣地區政治民主化. Taipei.

Huang Ying-che 黃英哲 2007: De-Japanisierung und Sinisierung - Der Wiederaufbau der taiwanesischen Kultur nach dem Zweiten Weltkrieg. 去日本化 在 中國化 - 戰後台灣文化重建 1945-1947. Taipei.

Hung Hsuan-fan 洪萱範 1994: Sprache, Gesellschaft und Bewußtsein des Volkes. Eine soziologische Studie über die Sprache in Taiwan. 語言社會與知識 - 台 灣語言社會學的研究. Taipei.

Hung Wei-jen 洪惟仁 1992. Die Krise der taiwanesischen Dialekte. 台灣語言危機. Taipei.

Hwang Jin-lin 黃金麟 1998: The Power of the Grotesque and the New Life Movement, 1934-1937. 醜怪的裝扮:新生活運動的政略分析. In: Taiwan: A Radical Quarterly in Social Studies. 台灣社會研究季刊. Vol. 30, 06. 1998. S. 163-203.

--2004: The Production of the Millitarized Body in China, 1895-1949. 近代中國的軍 事身體建構 (1895-1949). In: Bulletin of the Institute of Modern History Academia Sinica. 中央研究院近代史研究所集刊. Vol. 43，03. 2004. S. $173-221$.

--2009: Kriege, Körper, Modernisierung - Die militärische Verwaltung und Behandung der Körperlichkeit im modernen Taiwan (1895-2005). 戰爭 身體 
現代性 - 近代台灣的軍事治理與身體 (1895-2005). Taipei.

Hwang Kwang-kuo 黃光國 1983: Der konfuzianische Gedanke und die ostasiatische Modernisierung. 儒家思想與東亞現代化. Taipei.

Kang Tai-kuang (Hrsg.) 康代光編著 1965: Wachstum der Wirtschaft und Entwicklung der Erziehung. 經濟成長與教育發展. Taipei.

Kang Tai-kuang und Shen Tseng-chi 康代光, 沈曾圻 1965: Allgemeine Schilderung zur Koordination der Erziehung mit der Wirtschaftsentwicklung. 教育配合經 濟發展概論. Taipei.

Kao Cheng-i 高正儀 1985: Eine Analyse der Evolutionsgedanken von Herrn Sun Yat-sen. 中山先生進化思想析論. Magisterarbeit beim Institut für Studien über die „Drei Prinzipien des Volks” an der „National Taiwan University”. 國 立台灣大學三民主義研究所碩士論文.

Kao Chien-her 高健和 2006: Research on Taiwan's gereneral Education in The Authoritative Times of Civics Textbooks (1952-1989). 威權時期台灣初等教 育公民科教科書之研究 (1952-1989). Magisterarbeit beim Institut für Geschichte an der „Tam Kang University”. 淡江大學歷史研究所碩士論文.

Kao Ming 高明 1978: Aufsatz über die Entwicklung von „Chu-yin-fu-hao“, das römische Alphabetsystem für die Umschrift des Chinesischen und das Internationale Alphabetsystem. 論注音符號，國語羅馬與國際音標的演進. In: Hwa Kang Journal of Humanities. 台灣華岡文科學報. Vol. 11. January 1978 S. 315-346.

Kao Ming-shih 高明士 2005: Das Studium über die traditionellen Erziehung und das juristische System in Ostasien. 東亞傳統教育與法制研究. Taipei.

--2006: Kulturkreis und Erziehung in Ostasien. 東亞文化圈與教育. In: Himalaya Foundation (Hrsg.) 喜瑪拉雅基金會編. 2006: Chinesische Kultur und deren Interaktion und Fusion mit fremder Kultur. 中華文化與域外文化的互動與融 
合. Taipei. S. 181-215.

Kao Shao-i 高邵儀 1988: Chiang Kai-shek und die Jugend. 蔣中正與青年. Taipei.

King Yeo-chi 金耀基 1985: Von der Tradition zum Modernen. 從傳統到現代. Taipei.

Kong Ching-mao 孔慶茂 2008: „Pa-ku-wen“. 八股文史. Nan-king.

Ku Ming-yuen 顧明遠 主編 2000: Wörterbuch für Erziehungsereigenisse in der Welt. 世界教育大事典. Chiang-shu.

Ku Tao-chung 古道中 1985: Studie über die Politische Sozialisation der chinesischen Jugend. Eine Analyse am Fall der Freizeitveranstaltungen des „China Youth Corps“. 中國青年政治社會化之研究中國青年反共救國團自強活動個案. Magisterarbeit beim Institut für Politikwissenschaft an der Militärfachhochschule. 政治作戰學院政治學研究所碩士論文.

Kuan Mei-jung 管美蓉 2004: Aufnahmeprüfungen zur Universität und Bildung unter staatlicher Kontrolle - eine historische Unersuchung über die Wandlung des Aufnahmeprüfungssystems zur Universität auf Taiwan (1949- 2001). 大學 入學考試制度與教育控制臺灣地區的歷史考察 (1949-2001). Dissertation beim Insititut für Geschichte an der „National Cheng Chi University“. 國立政 治大學歷史研究所博士論文.

--2008: From Separation to Joint: Development of the Joint College Entrance Examination (1938-1940). 從自主到統一一抗戰前期大學入學考試的發展 (1938-1940). In: Bulletin of Academia Historica. 國史館學術集刊. Bd. 16, Juni 2008, S. 91-134.

Kuo Cheng-chao 郭正昭 1972: Sozialer Darwinismus und die Bewegung am Ende der Ching-Dynastie. 社會達爾文主義與晚清學會運動. In: Bulletin of the Instiute of Modern History Academia Sinica. 中央研究院近代史研究所集幵. Bd. III, December 1972, Taipei. S. 566-570. 
Kuo Shu-ying 郭樹英 2002: Das Verhältnis zwischen Aufhebung des kaiserlichen Beamtenprüfungssystems und Modernisierung - Ein entscheidender Wendepunkt der chinesischen Modernisierung. 科舉的廢止與我國現代化的 關係 - 中國現代化的一個關鍵性環節 -. In: Journal of Examination Yuen. 考銓季刊. Vol.20, 04. 2002 S. 90-112.

Kuo Ting-i 郭廷以 1987 : Selbststärkungsbewegung: Streben nach der westlichen Technik.自強運動：西洋技藝的追求. In: Fairbank, John K. (Hrsg.) 費正清 主編：Cambridge History of China: Late Ch’ing 1800-1911. Pt.1. 劍橋中國 史. 晚清篇 (上). Chinesische Ausgabe. Taipei.

--1988: Übersicht über die historische Ereignissen zu Taiwan. 台灣史事概說. Taipei.

Lai An-ming 來安民 1996: Die Entwicklung der Mittelschule auf Taiwan. 台灣中學 教育之演進. In: Hsu Nan-hao (Hrsg.) 徐南號主編 1996: Die taiwanesische Bildungsgeschichte. 台灣教育史. Taipei. S. 133-158.

Lai Hui-lan 賴惠蘭 1985 ：Das Studium über die Entsendung der Kinder in die USA am Ende der Ching-Dynastie. 清末留美幼童之研究. Magisterarbeit beim Institut für „Beziehungen zwischen China und den USA” an der „Chinese Culture University”. 文化大學中美關係研究所碩士論文.

Lai Tse-han 賴澤涵 1994: Untersuchungsberichte über den Aufstand am 28. Februar 1947.「二二八事件」研究報告. Taipei.

Lan Po-chou 藍博洲 1993: Der weiße Terror. 白色恐怖. Taipei.

Lee Deng-ker 李登科 1999: A Study of the Triangular Relations among Washington, Moscow, and Peking since 1949. 五十年的「中」、美、蘇(俄)關係. In: Mainland China Studies. 中國大陸研究. Vol: 42 (10), Oct. 1999, S. 45-68.

Lee Han-yun 李漢雲 2003: Die Geschichte des modernen China. 中國近代史. Taipei. 
Lee Tai-han 李泰翰 2002: Parteiapparat, Militär und Erziehung - eine Untersuchung über die Einführung der militärischen Erziehung in den 1950er Jahren. 黨團、 軍事與教育 - 一九五 0 年代學生軍訓進入校園之研究. Magisterarbeit beim Insititut für Geschichte an der „National Central University“. 國立中央大學大 學歷史研究所碩士論文.

Lee Thomas H.C. 李弘祺 1993: Die amtliche Schule und das kaiserliche Beamtenprüfungssystem. 宋代官學教育與科舉.Taipei.

--2006: Tradition der Erziehung in China und Ostasien Bd. 1 - Die Erziehung und das kaiserliche Beamtenprüfungssystem in China. 中國與東亞的教育傳統 (一)：中國的教育與科舉. Taipei.

Lee Wei-sung 李偉松 2005: Studie über Chang Ching-kuo und „the China Youth Corps“ (1969-1988). 蔣經國與救國團之研究（1969-1988). Magisterarbeit beim Insititut für Geschichte an der „National Central University“. 中央大學 歷史研究所碩士論文.

Li Ao 1962 (a): Stellungnahme zu den Äußerungen über die östliche und westliche Kultur. 給談中西文化的人看看病 In: Zeitschrift Wen-hsing. 文星雜誌 Heft 52,1962, S.9-12.

--1962 (b) : Hu Shi - ein Sämann 播種者胡適. In: Zeitschrift Wen-hsing. 文星雜誌. Heft 51, 1962, S.3-4.

Li Chih-kang 李志剛 1992: Sammelband: Das Christentum und die chinesische Kultur in der modernen Zeit. 基督教與近代中國文化論文集. Taipei.

Li Chin-hsi 黎錦熙 1931: Kurze Schilderung über die Erstellung des römischen Umschriftsystems für die chinesische Sprache. 國語羅馬字公布經過述略. Peking.

Li Ching-erh 李靜兒 2003: 50 Jahre Erziehung der Überseechinesen (1950-2000). 近 半世紀的華僑教育 (1950-2000). Taichung. 
Li Chuan-ling 李俊霖 2006: Pädagogische Gedanken und Praxis von Chang Chi-yun. 張其昀之教育思想與實踐. Magisterarbeit beim Institut für Politikwissenschaft an der „National Taiwan Normal University“. 國立臺灣師範大學政治學研究 所碩士論文.

Li Hsi-suo 李喜所 1987: Die modernen chinesischen Auslandsstudenten. 近代中國 的留學生. Peking.

Li Hsiao-feng 李篠峰 1991: Heraustreten aus dem Schatten am 28. Februar 1947. 走 出二二八的陰影. Taipei.

Li Hsiao-feng und Liu Feng-sung 李䈗峰、劉峰松 1994: Das Lesen über taiwanesische Geschichte. 台灣歷史閱覽. Taipei.

Li Hsin-ta 李新達 1995: Geschichte des chinesischen Beamtenprüfungssystems. 中國 科舉制度史. Taipei.

Li Kuan-chih 李光智 2006: Aufbau und Entwicklung der staatlichen Lehrpläne in den „öffentlichen Schulen“ auf Taiwan unter der japanischen Besatzungszeit. 台灣日治時期公學校課程的形成與發展. Magisterarbeit beim Institut für Politikwissenschaft an der „National Sun Yatsen University“. 國立中山大學 政治研究所碩士論文.

Li Kou-ting 李國鼎 1961: „Development of Human Resources and 1st Relations with Science Education“. In: Industry of Free China, Vol. XVI, No. 5, Nov. 1961.

--1962: „How to Increase Employment“. In: Industry of free China, Vol. XVIII, No.2 Aug. 1962.

--1978: Die Erfahrungen des raschen Wirtschaftswachstums auf Taiwan. 台灣經濟快 速成長的經驗. Taipei.

--1987: Die Prinzipien und Strategien der Wirtschaftsentwicklung. In: Li Kou-ting und Chen Za-mu 李國鼎、陳在木 (Hrsg.) 1987: Allgemeines zur Strategie der Wirtschaftsentwicklung in Taiwan. 我國經濟發展策略總論. Taipei. 
--1994: Wirtschaftsentwicklung und Arbeitskräfte in Taiwan. 經濟發展與人力資源. In: Ders.1994: Probleme im Bezug auf Arbeitskräfte und Bevölkerung in Taiwan. 台灣的人力資源與人口問題. Nanking.

--1999: Die technische Ausbildung und Arbeitskräfte der Techniker innerhalb der Wirtschaftsentwicklung in Taiwan. 台灣經濟發展中的科技教育與人才. Taipei.

Li Yuen-huei 李園會 1983: Die elementare Bildung unter der japanischen Besatzung in Taiwan. 日據時期台灣初等教育制度. Taipei.

--1984: Studie über die Bildungspolitik zu Beginn der Rückgabe an China und die Übersiedlung der KMT-Regierung auf Taiwan. 台灣光復時期與政府遷台初 期教育政策之研究. Kaohsiung.

--2005: Die taiwanesische Bildungsgeschichte unter der japanischen Besatzungszeit. 日據時期臺灣教育史. Taipei.

Liang Chi-chao 梁啟超 1897: Aufsatz über die „Frauenerziehung“. 論女學. In: Gesamtwerk Liang Chi-chaos 梁啟超全集 1960: Sammelband der Aufsätze I. Taipei. S. 34-37.

--1920: Reiseberichte in Europa. 歐遊心影錄. Neue Ausgabe 1959. Taipei.

Liang Shu-ming 梁漱溟 2002: die „Östliche und westliche Kultur und ihre Philosophie“. 東西文化及其哲學. Taipei.

Lin Ben 林本 1977: Achtzig Jahre Umwandlung des Bildungswesens. 學制演變八十 年. In: Journal of normal University Taiwan. 启大學報. Bd. 22, 1977, S. 1-36.

Lin Cheng-chen 林正珍 1994：晚清學制的變遷及西方教育制度的引介. In: Journal of National Tsing Hua University for Genenal Education. 清華大學通識教育 季刊. Vol.1 (4). Dec.1994, S. 45-48.

Lin Cheng-chen und Lin Hung-chun 林正珍和林鴻鈞 2002: Pointed Criticism from Europe: From the League of Nations' Commission of Survey of Chinese 
Education with Its Focus on the Change of Chinese Educational System in 1930s. 國聯中國教育考察團與一九三零年代中國教育制度的變遷. In: Journal of Humanities College of Liberal Arts National Chung Hsing University. 興大人文學報. Vol 32, Juni 2002, S. 835-862.

Lin Chian-fu 林建福 1996: Die Entwicklung der Hochschulbildung in Taiwan. 台灣 高等教育之演進. In：Hsu Nan-hao (Hrsg.) 1996: Die taiwanesische Bildungsgeschichte. Taipei. S. 159-184.

Lin Chiou-huang 林丘湟 2006: The provincial discriminating system in economic side and ethnicity construction by KMT-regime. 國民黨政權在經濟上的省籍差別 待遇 體制與族群建構. Magisterarbeit beim Institut für Studien über die Lehre von Sun Yat-sen an der „National Sun Yat-sen University”. 國立中山大學中 山學術研究所.

Lin Chuan-i 林全義 2002: Studie über die Aufnahmemethode zur Senior High School in Taiwan. 台灣地區高級中學入學方式之研究. Magisterarbeit beim Institut für Politikwissenschaft an der „National Kaohsiung Normal University“. 國立 高雄師範大學教育學系碩士論文.

Lin Gou-shian 林果顯 2001: Untersuchung über die Kommission der Durchführung für die "Bewegung zur Restauration der chinesischen Kultur"(1966-1975).Aufbau und Umwandlung der Sanktion der Herrschaft. 「中華文化復興運動推 行委員會」之研究 (1966-1975) - 統治正當性的建立與轉變. Magisterarbeit beim Insititut für Geschichte an der „National Cheng Chi University“.

Lin Hsin-fa1996 林新發 1996: Der fremde Einfluss auf die Entwicklung des Bildungswesens - Kommentar über „Anwendung der Theorie von Modernisierung und Abhängigkeit“ -. 我國教育發展的外來影響一「現代化理論與依賴 理論之應用」評論. In: Bildungsreform - von Tradition zu Postmodern. 載於教 育改革一從傳統到後現代. Taipei. 
Lin Pao-tsung und Chen Cheng-mao (Hrsg.) 林寶琮 陳正茂編 2007: Grundriss der taiwanesischen Geschichte. 台灣史綱. Taipei.

Lin Yong-feng 林永豐 1996: Die Entwicklung der taiwanesischen Lehrerausbildung. In: Hsu Nan-hao 1996 徐南號 Die taiwanesische Bildungsgeschichte. 台灣教 育史 Taipei. S. 33-58.

Liu Chen 劉真 1979: Die Kritik der quantitativen und qualitativen Erziehungsprobleme in der Provinz Taiwan. 本省教育量與質的問題之檢討. Taipei.

Liu Li-hui 劉麗慧 2000: Das Studium über das Ideal der Allgemeinbildung Herr Chung-shan's 中山先生普及教育理想之研究 $\mathrm{In}: \mathrm{Fu} H \operatorname{Hing} \mathrm{Kang}$ Academic Journal. 復興崗學報. Vol.70, September 2000. S. 1-22.

Liu Long-hsin 劉龍心 2002: Akademie und System: das akademische System und der Aufbau der modernen chinesischen Geschichtsforschung. 學術與制度：學科 體制與現代中國史學的建立. Taipei.

Liu Hai-feng 劉海峰 2006: Die Tradition der kaiserlichen Beamtenprüfung und die kulturelle Tradition der Prüfung in Ostasien. 科舉傳統與東亞考試文化圈. In: Lee Thomas H.C. (Hrsg.) 李弘祺編.2006: Tradition der Erziehung in China und Ostasien (1) : Die Erziehung und das kaiserliche Beamtenprüfungssystem in China. 中國與東亞的教育傳統 (一) : 中國的教育與科舉. Taipei. S. 461-486.

Liu Hong 劉虹 2006: Die moderne Interpretation über den Werdegang von Pa-ku-wen von Wang An-shi in der Nord Song-Dynastie bis zur Zeit des Hong Wu Kaisers in der Ming-Dynastie. 從北宋王安石經義取士到明洪武八股文 形成的當代詮釋. In：Lee Thomas H.C. (Hrsg.) 2006: Die Erziehungstradition in China und Ostasien (1): Die Erziehung und das kaiserliche Beamtenprüfungssystem in China. 中國與東亞的教育傳統 $(一)$ ：中國的教育與科舉. Taipei. S. 71-118. 
Liu Hsien-yun 劉先雲 1956: Der Prozeß der Durchführung der Abschlussprüfung in der Senior High School. 辦理高中畢業生會考之經過. In: Erziehung und Kultur. 教育與文化. Vol 14 (4), 1956, S. 13.

Liu Hui-hsuan 劉惠璇 1986: Die Gesellschaft für die Entwicklung der „neuen Erziehung“ und „Bewegung der Autonomie der Erziehung“. 中華教育改進社 與教育獨立運動 (1921-1927). Magisterarbeit beim Institut für Geschichte an der „National Taiwan Normal University“. 國立台灣師範大學歷史研究所碩 士論文.

Liu Ke-chih 劉克智 1975: Zuwachs und Einsatz von Arbeitskräften in Taiwan. 台 灣人力資源的成長與運用. Taipei.

Liu Shu-hsien 劉述先 1997: Die Entwicklung des neuen Konfuzianismus. 新儒學的 開展. S. 54-60. Tai-chung .

Liou Te-mei 劉德美 1989: Die Entwicklung von Bildungspolitik, Einstellung zum Studium und Bildungswesen in der Ching- Dynastie. 清季的學政與學風、學 制的演變. In: Journal of National Taiwan Normal University, Institute for History. 台灣所大歷史學報. Vol. 17, 1989, S. 301-340.

Lo Li-wen 羅俐雯 2002: Die Reform der taiwanesischen Demokratie anhand der These von Samuel P. Huntington. 台灣民主轉型 (1980-2000) 之研究一以 Samuel P. Huntington 的理論作驗證. Magisterarbeit beim Insitiut für Politikwissenschaft an der „Chinese Culture University“. 文化大學政治研究 所碩士論文.

Lo Wen-hao 羅文豪 2005: The Decision Process of the Kennedy Administration Urge - Self-restraint on both sides of the Taiwan Strait in 1962. 甘迺迪政府要 求兩岸降溫自制之決策過程 (1962). Magisterarbeit beim Institut für US-America Studie an der „Tam Kang University“. 淡江大學美國研究所碩 土論文 
Lo Tzu 羅滋 1981: Der chinesische Nationalismus und die Bewegung gegen das Christentum in den 1920er Jahren. 中國民族主義與 1920 年代之反基督教運 動. In: Sammelband der Aufsätze: Die Geschichte des modernen China. 中國 現代史論集. Bd. 6. Taipei. S. 211-234.

Lu Fang-sang 呂芳上 1976: Chiang Kai-shek und die Gründung der Whampoa Militärakademie in Kanton. 總統蔣公與黃埔軍校的創建. In: Journal of National Taiwan Normal University on Institute for History. 台灣猏历大歷史學 報. Heft 4, 1976, S. 37-51.

--1994: Von der Studentenbewegung bis zur Mobilisierung der Studenten von 1919 bis 1929. 從學生運動到運動學生一民國八年至十八年. Taipei.

Lu Shu-huei 陸淑慧 2006: Studium über den Gedanken zur Wirtschaft von Huang Tsung-shi. 黃宗羲( 1610-1695) 經濟思想之探究. Magisterarbeit beim Institut für Geschichte an der „National Chen-Chi University“. 國立政治大學歷史系 碩士論文.

Luoh Ming-Ching 駱明慶 2002: Who are NTU Students? - Differences across Ethnic and Gender Groups and Urban/Rural Discrepancy. 誰是台大學生? 省籍和城鄉差異, 性別. In: Taiwan Economic Review 經濟論文叢刊 30:1, 2002, S. 113-147.

Masahisa Shimura 志村雅久 1993: Studien über die Verbreitung der Nationalsprache im Gebiet Taiwan von ROC. 中華民國台灣地區推行國語運動之研究. Magisterarbeit beim Institut für Studien über die „Drei Prinzipien des Volkes“an der „National Taiwan University“. 國立台灣大學三民主義研究所 碩士論文.

Pan Tai-hsiung 潘台雄 1995: Der Gedanken der politischen Reform von Kang You-wei und Liang Chi-chao (1898-1911).康有為與梁啟超的君主立憲思想 (1898-1911). Dissertation beim Institut für Politikwissenschaft an der „National 
Chen-chi University“. 國立政治大學政治研究所博士論文.

Peng Ta-nien 彭大年 2005: Das vergessenene Kriegsprojekt Kou-kuang. 塵封的作 戰計畫:國光計畫. Taipei.

Sang Bing 桑兵 1991: Die Schüler in der modernen Schule in der späten ChingDynastie und der soziale Wandel. 晚清學堂學生與社會變遷. Taipei.

Shen Ting-ping 沈定平 1994: Die Verbreitung der westlichen Kenntnisse und die frühe Modernisierung Chinas. 西學的傳播與中國的早期近代化. In: Lin Chih-ping (Hrsg.) 林治平主編 1994: Das Christentum und die Modernisierung Chinas. 基督教與中國現代化. Taipei. S. 87-117.

--2001: Der Kulturaustausch in der Zeit des Übergangs zwischen Ming- und Ching-Dynastie: Anpassung und Verständnis in der Ming-Dynastie. 明清之際 中西交流史：明代 - 調適與會通. Peking.

Shih Chi-sheng 1993 石計生: Ideologie und taiwanesische Lehrbücher. 意識型態與 台灣教科書. Taipei.

Shih Chih-hui 施志輝 1995: Studie über die „Bewegung zur Bewahrung und Restauration der chinesischen Kultur“ (1966-1991). 中華文化復興運動之研 究 (1966-1991). Magisterarbeit beim Institut für Geschichte an der „National Taiwan Normal University“. 國立台灣師範大學歷史研究所碩士論文.

Shih Min-fa 施明發 1996: Die Entwicklung der Berufsausbildung auf Taiwan. 臺灣 職業教育之演進．In：Hsu Nan-hao (Hrsg.) 徐南號主編 1996：Die taiwanesische Bildungsgeschichte.台灣教育史. Taipei. S. 85-132.

Shih Ming-hsiung 施明雄 1998: Die dunkle Zeit unter dem weißen Terror: die Geschichte des Leidens der Taiwanesen. 白色恐怖黑暗時代：台灣人受難史. Taipei.

Shih Ying-chun 史穎君 1984: Studie über die „Bewegung der Verbreitung der Nationalsprache“ (1912-1981). 我國國語運動之研究 (1912-1981). Magister- 
arbeit beim Institut für Pädagogik an der „National Cheng Chi University”. 國 立政治大學教育研究所碩士論文.

Shu Hsin-cheng 舒新城 1989: Die Geschichte des Auslandsstudiums im modernen China. 近代中國留學史. Shang Hai.

Su Cheng-pei 蘇正沛 2005: Dominance and Resistance-A Comparison of "Free China” and “Formosan Magazine” Events. 支配與反抗-『自由中國』事件與 『美麗島』事件之比較. Magisterarbeit beim Institut für Studium über die Lehre von Sun Yat-sen an der „National Sun Yat-sen University“. 國立中山大 學中山學術研究所碩士論文.

Sun Chiang-lin 孫家麒 1961: Die Konspiration vom Aufbau eines Systems der Spionageorganisation durch Chiang Ching-kou. 蔣經國建立台灣特務系統密 辛. Taipei.

Su Ching-hsuan 蘇慶軒 2008: State-Building and the White Terror：The Causes of Political Persecution in the early 1950s in Taiwan. 國家建制與白色恐怖：五 ○年代初期臺灣政治案件形成之原因. Magisterarbeit beim Institut für Politikwissenschaft an der „National Taiwan University“. 國立台灣大學政治 研究所碩士論文.

Su Rui-qiang 蘇瑞鏘 2003: Studie über die Gründungsbewegung der demokratischen Partei Chinas.「中國民主黨」組黨運動之研究. Magisterarbeit beim Institut für Geschichte an der „National Taiwan Normal University“. 國立台灣師範大 學歷史研究所碩士論文.

Su Yun-fung 蘇雲峰 1981:Der Wandlung der Erziehungskonzepte im modernen China. 近代中國教育思想之演變. In: Bulletin of the Institute of Modern History Academia Sinica. 中央研究院近代史研究所集刊.. Vol.10, 1981, S. $1-31$.

--2005: Beginn und Erweiterung der neuen Erziehung in China 1860-1928. 中國新教 
育的萌芽與成長 1860-1928. Taipei.

Su Yu-kuo 蘇玉國 1998: Studie über die „Bildung des Volkgeistes“ im Fach Chinesisch in der Junior High School in Taiwan. 我國國民中學國文科民族精 神教育之研究. Magisterarbeit beim Chung-Shan Institut für Geistes- und Sozialwissenschaft an der „National Cheng Chi University“. 國立政治大學中 山人文社會科學研究所碩士論文..

Sun Pang-cheng 孫邦正 1976 : Die Erziehungsgedanken von Präsident Chiang Kai-shek. 蔣公的教育思想. In: Journal of National Taiwan Normal University. 台灣師大學報. Vol.21, 1976, S. 27-49.

--1970: Kommentar über die „Reformvorschläge des Briten, R.H. Tawney für das chinesische Bildungswesen. 談英人陶納對中國教育的改革意見. In: Ders. 1970: Die Erziehungsprobleme Chinas. 中國教育問題. Taipei. S. 67-70.

Song Ten-zheng 1995: „Großes Lernen“ (Ta-hsue 大學篇). In: Wang Yun-wu (Hrsg.) 1995: Die heutige Interpretation und „Kommentar von vier Büchern“. Taipei. S. $1-40$.

Tai Chi-tao 戴季陶 1931:,Einführung für den gesamten Bericht über die erstjährige Durchführung der Aufnahmeprüfung der Beamten“第一屆考試制度實施總體 報告 In: Institution für das Prüfungs- und Auswahlsystem (Hrsg.) 1984 銓叢書 指導委員會: Tai Chi-tao und das Prüfungs- und Auswahlsystem. 戴季陶先生 與考銓制度. Taipei.

Tai Pao-tsun 戴寶村 2006: Die politische Geschichte Taiwans. 台灣政治史. Taipei. Tang Chih-min 湯志民 2002: School Buildings in Taiwan.台灣的學校建築. Taipei.

Teng Hung-po 鄧洪波 2005: Die Geschichte der chinesischen „Akademien“ (Shuyuen 書院). 中國書院史. Taipei.

Ting Shih-ta 丁時達 (2005): Studie über die Nachhilfe-Institute zur Vorbereitung für die Aufnahmeprüfung zur Hochschule in Taipei. 臺北市升大學文理補習班變 
遷研究. Magisterarbeit beim Institut für Pädagogik an der „National Taiwan Normal University“. 國立臺灣師範大學教育研究所碩士論文.

Tong Tekong 唐德剛 2005: Die Autobiographie Hu Shi. 胡適口述自傳. Taipei.

Tsai Ming-hsien 蔡明賢 2009: Die Sprachpolitik in Taiwan nach dem Zweiten Weltkrieg (1945-2008). Von der Bewegung der Nationalsprache zur Bewegung der Muttersprache. 戰後台灣的語言政策 (1945-2008) - 從國語運動到母語 運動. Magisterarbeit beim Institut für Geschichte an der „National Chung Hsien University“. 國立中興大學歷史學系碩士論文.

Tsai Pei-huo 蔡培火等 1971: Die Geschichte der Volksbewegung des modernen Taiwan. 台灣近代民族運動史. Taipei.

Tsai Yuen-pei 蔡元培 1912: Meinungen zur „neuen Erziehung“. 對于新教育之意見. In: Sammelband Tsai Yuen-peis 蔡元培全集 1984, Bd. 2. Taipei. S. 130-137. --1922: Aufsatz über die Autonomie der Erziehung. 教育獨立議. In: Sammelband der Aufsätze Tsai Yuen-peis. 蔡元培文選. 1994. Shang Hai. S. 352-354

Tsao Chang-jen und Lin Chung-yao 曹常仁、林崇堯 2003: Der Pionier der modernen Berufsausbildung Chinas - Studium über Konzepte der beruflichen Ausbildung von Huang Jen-pei. 近代中國職業教育開拓者-黃炎培職業教育 理念之初探. In: Quarterly Journal of Technological and Vocational Education. 技術及職業教育隻月们. Bd. 78. 2003, S. 38-41.

Tsao Ruey-tai 曹瑞泰 2007: A Contrast and Comparison Study on the Evolution Process of Characters and Pronunciation in Chinese and Japanese Language. 中日語言文字與語音發展過程之對比分析. In: Journal of the Chinese for General Education. 通識研究集刊. Kai Nan University. 開 南 大 學. Vol. 11, 06. 2007, S. 1-24.

Tsao Yung-hen 曹永和 1979: Taiwan unter der Besatzung von der Holländer und 
Spanier.荷蘭與西班牙佔據時期的臺灣. In: Ders. 1979: Studie über Taiwan in der früheren Zeit. 臺灣早期歷史研究. Taipei. S. 25-44.

Tseng Chien-min 曾健民 2009: 1949 Der Chinesische Bürgerkrieg und Taiwan. 1949 國共內戰與台灣. Taipei.

Tseng Hui-wen 曾蕙雯 1999: Studie über die elementare Erziehung in Taiwan während der Ching-Dynastie (1684-1895). 清代台灣啟蒙教育研究 (1684-1895). Magisterarbeit beim Institut für Pädagogik an der „National Taiwan Normal University“. 國立台灣師範大學教育研究所碩士論文.

Tsung Liang-tung 宗亮東 1986: Sammelband von Ansätze über Pädagogische Beratung und Pädagogik. 輔導與教育論文選集. Taipei.

Tu Chian-feng 杜劍峰 1989: Die Propagierung der Ideologie von den „Drei Prinzipien des Volkes“ - Tai Chi-tao. 三民主義意識型態的肇建者-戴季陶. Magisterarbeit beim Institut für Studie über die Lehre von Sun Yat-sen an der „National Sun Yat-sen University“. 國立中山大學中山學術研究所.

Tu Hsueh-yuen 杜學元 1997: Die Frauenerziehung Liang Chi-chao während der Hundert-Tage-Reform. 維新時期梁啟超的女子教育思想. In: Journal of Southwest China Normal University. 西南所範大學學報. Vol. 5. 1997, S. 89-91.

Wakabayashi Masahiro 若林正丈 1997: Taiwan: Getrennter Staat und Demokratisierung. 台灣:分裂國家與民主化. Chinesische Ausgabe. Taipei.

Wan Chih-ting 汪知亭 1959: Die Geschichte der taiwanesischen Bildung. 臺灣教育 史. Taipei.

Wan I-ting 汪彝定 1991: Nach der Wendezeit. 走過關鍵年代. Taipei.

Wang Bing-chao 王炳照 1994: Die Bildungsgeschichte des modernen China. 中國近 代教育史. Taipei.

Wang Bing-chao und Hsu Yung 王炳照、徐勇主編 2002: Studie über das kaiserliche 253 
Beamtenprüfungssystem. 中國科舉制度研究. Shih Chia Chuang: He Pei.

Wang Chia-tong und Wu Yuh-yi 王家通、吳裕益 1983: Analyse der Quote der aufgenommenen Studenten aus den „Star Senior High School“. 明星高中錄取 率之分析. In: Education information digest. 教育資料文摘. November 1983. S. 35-39.

Wang Chih-ping 王峙萍 2002: Maze of Uprising and Resistance-Student Movements in the $2 / 28$ Incident. 暴動與抗爭的迷思--論二二八事件中的校園與學生反 抗運動. Magisterarbeit beim Institut für Geschichte an der “Tam Kang University”. 淡江大學歷史研究所碩士論文.

Wang Chih-hsiang 王之相 2008: Die Entstehung des taiwanesischen modernen Staats 台灣現代國家的興起. Taipei.

Wang Chin-chueh 王錦雀 2002: The Evolution of Colonial and Policies of Education during the Period of Japanese Ruling on Taiwan. 日本治台時期殖民與教育政 策之演變日本治臺時期殖民與教育政策之演變. In: Bulletin of Civic and Moral Education. 公民訓育學報. Vol.11,02.2002, S. 131- 157.

--2005: Die Staatsbürgererziehung und die Eigenschaften der Staatsbürger auf Taiwan unter der japanischen Besatzungszeit. 日治時期台灣公民教育與公民特性. Taipei.

Wang Chung-fu 王仲孚 1981: Wandlung der Kultur im modernen China. 現代中國 文化的轉變. In: Chin Hsiao-i (Hrsg.) 秦孝儀編 1981: Die kulturelle Entwicklungsgeschichte Chinas. 中國文化發展史. Bd.2. S.501-835. Taipei.

Wang Er-min 王爾敏 1969: Die These „chinesische Weltanschauung als Grundlage, westliches Wissen zum praktischen Gebrauch“" von Intellektuellen in der Ching-Dynastie. 清季知識份子的中體西用論. In: Ders. 1969: Ansätze über die politischen Gedanken der späteren Ching-Dynastie. 晚清政治思想史論. Taipei. S. 51-71. 
--1982: Die Verbreitung des modernen Wissens, Aufklärung und Bewegung der Nationalsprache. 中國近代知識普及化之自覺及國語運動. In: Bulletin of the Institute of Modern History Academia Sinica. 中央研究院近代史研究所集刊. Vol.11, 07.1982, S. 13-45.

Wang Feng-chieh 王鳳㫮 1998: Die Geschichte der chinesischen Erziehung. 中國教 育史 Taipei.

Wang Hong-zen 王宏仁 1999: Studie zur Struktur und Mobilität der sozialen Schichten in den 1950er Jahren. 一九五 O 年代的台灣階級結構與流動初探. In. Taiwan: A Radical Quarterly in Social Studies. 台灣社會研究季刊. Bd. 36. Dec. 1999, S. 1-43.

Wang Jenn-wu und Ling Wen-ing 王震武、林文瑛 1994: Aufnahmesystem und Symptome von ,Sheng-hsueh chu-i“升學制度與升學症候群. In: Taiwan Research Fund (Hrsg.) 台灣研究基金會編: Bildungsreform in Taiwan. 台灣 的教育改革. Taipei. S. 502-539.

Wang Jenn-wu und Ling Wen-ing 王震武、林文瑛 1996 (a): Kontrolle und Normalisierung oder Fehlentwicklungen in der taiwanesischen Bildungspolitik Integrierte Studie über Prinzipien der Deregulation in der Bildung. 台灣教育的 管制、規範或扭曲——鬆綁」原則整合研究. Taipei.

--1996 (b): Eine Anaylse der sozialen Effekte der Differenzierungsmaßnahmen. 分流 教育影響之分析. Taipei.

Wang Liang-ching 王良卿 2003: Reform in schwankender Zeit - Reorganisation der KMT (1945-1950). 動盪中的改革-中國國民黨從革新走向改造 (1945-1950). Dissertation beim Institut für Geschichte an der „National Cheng Chi University“. 國立政治大學歷史系博士論文.

Wang Lun-shen 王倫信 2002: Studie der Mittelschulerziehung am Ende der ChingDynastie und zu Beginn der Republik China. 清末民國時期中學教育研 
究. Shang Hai.

Wang Pei-lin 王佩玲 2001: Umweltevaluation und Umwandlung der Institution des „China Youth Corps“(1987-2001). 環境演化與救國團之組織變遷--救國團 (1987-2001). Magisterarbeit beim Institut für Politikwissenschaft an der „National Taiwan Universty“. 國立台灣大學政治研究所碩士論文.

Wang Shiao-pao 王曉波 1988: Taiwanesische Geschichte und Taiwanesen. 台灣史 與台灣人. Taipei.

Wang Shou-nan 王壽南 1981: „Die Bewegung zur Bewahrung und Restauration chinesischer Kultur“. 中華文化復興運動. In: Chin Hsiao-I (Hrsg.) 秦孝儀編 1981: Die kulturelle Entwicklungsgeschichte Chinas. 中國文化發展史. Bd.4. Taipei. S.2027-2201.

Wang Wen-lung 王文隆 2008：An Imaginary Motherland in the Geographic Textbook of Taiwanese Middle Schools (1949-1999). 臺灣中學地理教科書的 祖國想像 (1949-1999). In: Bulletin of Academia Historica. 國史館學術期刊. Vol. 17. Dec. 2008, S.201-251.

Wen Chung-i 文崇一 1988: Der Charakter des chinesischen Volkes anhand ihrer Werte und Normen. 從價值取向談中國國民性. In: Li I-yuen und Yang Kuo-shu 李亦園、楊國樞主編 1988: Die Persönlichkeit der Chinesen.中國人 的性格. S.49-84.

Wen Hsing-ying 文馨瑩 1989: US-aid and the dependent Development of Taiwan (1951-1965). 經濟奇蹟的背後一台灣美援經濟的政經分析(1951-1965). Magisterarbeit beim Institut für Politikwissenschaft an der „National Taiwan University“. 國立台灣大學政治研究所碩士論文.

Wu Chan-liang 吳展良 1999: A New Interpretation of the Intention and Connotation of Yen Fu's “On Evolution”. 嚴復《天演論》作意與內涵新詮. In: Bulletin of the Department of History of National Taiwan University. 台大歷史學報. 
Vol.24, Dec.1999, S. 103-176.

Wu Chia-ying 吳家瑩 1990: Entwicklungsgeschichte der Bildungspolitik der Republik China unter dem KMT-Regime 1925-1940. 中華民國教育政策發展 史一國民政府時期 (1925-1940). Taipei.

Wu Mi-Cha 吳密察 1991: Die Errichtung der Taihoku Imperal Universität im Zusammenhang von Entwicklung des Bildungssystems unter japanischer Kolonialherrschaft. 從日本殖民地教育學制發展看台北帝國大學的設立. In: Wu Mi-Cha 1991: Studien über die moderne Geschichte Taiwans. 台灣近代史 研究. Taipei. S.149-175.

Wu Wen-hsing 吳文星 1978: Studie über die „konfuzianische Schule“ unter der japanischen Besatzung. 日據時代臺灣書房之研究. In: Denken und Reden. 思 與言. Band 16. Nr.3, 1978, S.264-291.

--1992: Studie der Führungsschichten in der taiwanesischen Gesellschaft unter der japanischen Besatzung. 日據時期臺灣社會領導階層之研究. Taipei.

--2000: Soziale Mobilität und Bildung unter der japanischen Besatzung. 日治時期台 灣的教育與社會流動. In: Taiwan Wen Hsien. 台灣文獻. Vol. 51(2) 06. 2000. S.163-173.

--2003: Den Kenjiro und das Schuledikt zu Taiwan 1922. 田健治郎與 1922 年臺灣 教育令 In: China und die Tradition der Shu-yuen in Ostasien (2)- Die Tradition der Shu-yuen und die Wendepunkte zur modernen Erziehung. 中國與東亞的書 院傳統(二)-東亞的書院傳統與近代教育的轉折. Taipei. S.527-547.

Yanaihara Tadao 矢內原忠雄 1987: Taiwan unter dem japanischen Imperialismus. 本帝國主義下之台灣. Chinesische Ausgabe. Taipei.

Yang Chen-chien und Chin Tao-sheng 楊成鑒、金濤聲 (1995): Das chinesische Prüfungswesen.中國考試學. Taipei.

Yang Chi-tung 楊啟棟 1994: Die Einflüsse des gewerblichen Ausbildungssystems 
auf die Entwicklung der beruflichen Ausbildung. 我國工業職業教育對職業訓 練發展之影響. In: Bulletin of the National Institute of Education Materials. 教 育資料集刊. Bd. 19. 1994, Taipei. S. 145-160.

Yang Hsueh-wei 楊學為編 (Hrsg.): Sammelband: Historische Dokumente des chinesischen Prüfungssystems . 中國考試制度史資料選編. He Fei:合肥市

Yang Kuo-shu und Ye Chi-cheng 楊國樞、葉啟政 1978: Erziehungsprobleme unter „Sheng-hsueh chu-i““升學主義下的教育問題. In: Ders. (Hrsg.) 楊國樞 葉 啟政主編: „Soziale Probleme Taiwans“. 臺灣的社會問題 Taipei. S. 73-93.

Yang Kuo-tzu 楊國賜 1981 (a): Bildung. In: Geschichte der kulturellen Entwicklung der Republik China. Bd.2. Taipei. S. 663-844.

-- 1981 (b): Die Wandlung der Bildungspolitik und deren Ergebinsse. 教育政策的演 變與其績效. S.83-87. In: Die Erziehung seit den 70 Jahren ab der Gründung der Republik China. Bd. 1. 中華民國開國七十年之教育 (上). Taipei.

Yang Mao-chun 楊稿春 1988: Der Familialismus Chinas und die nationale Persönlichkeit. 中國的家族主義與國民性格. Taipei.

Yang Rong-cong 楊榮聰 1992: Kulturelle Konstruktion und nationale Identität: Das sinisierte Taiwan nach dem Zweiten Weltkrieg. 文化建構與國族認同:戰後台 灣的中國化. Magisterarbeit beim Institut für Soziologie und Anthropologie an der „National Tsing Hua University“. 國立清華大學社會人類學研究所碩士 論文.

Yang Tsui-hua 1978 楊翠華: Die antireligiöse Erziehung und Rücknahme der Erziehungshoheit (1922-1930). 非宗教教育與收回教育權運動 (1922-1930). Magisterarbeit beim Institut für Geschichte an der „National Chengchi University“. 國立政治大學歷史研究所碩士論文.

--1989: Die Gründung und Umorganisation der „China Foundation for the Promotion of Education and Culture“. 中基會的成立與改組. In: Bulletin of the Institute 
of Modern History Academia Sinica. 中央研究院近代史研究所集刊. Vol.18, 06. 1989 , S. $259-280$.

--1991: Die finaziellle Unterstützung der „China Foundation for the Promotion of Education and Culture" für die Entwicklung der Wissenschaft in China. 中基 會對科學的贊助.

--1995.: Jen Hung-chuan und die wissenschaftlichen Gedanken und Aktivitäten im modernen China. 任鴻鹪與中國近代科學思想與事業 In: Bulletin of the Institute of Modern History Academia Sinica. 中央研究院近代史研究集刊. Vol.24 (1), Juny 1995, S. 297-324.

--1999: Wang Shih-chian und der wissenschaftliche Austausch zwischen der Republik China und den USA von 1963 bis 1978: Hilfe oder Zusammenarbeit?王世杰與 中美科學學術交流，1963-1978：援助或合作？ In: A Journal of European and American Studies. 歐美研究. Vol. 29 (2), Juny 1999, S. 41-103.

--2002: Beitrag von Hu Shih zur Entwicklung der Wissenschaft auf Taiwan - Der verlängerte Traum von der „Autonomie der Forschung“. 胡適對台灣科學發展 的推動：「學術獨立」夢想的延續. In: Research in Chinese Studies. 漢學研 究. Vol.20:2, Dec. 2002, S. 327-352.

--2003: Vorläufer der Technologiepolitik in Taiwan: Wu Ta-you und „Direct Council of Development of Science“. 臺灣科技政策的先導：吳大猷與科導會. In: Taiwan Historical Research. 台灣史研究. Vol.10 (2), Dec.2003, S. 67-110.

Yen Chia-chih 葉家熾 1981: Religion und der chinesische Nationalismus - die theoretische Basis der Antireligion von Gelehrten zu Beginn der Republik China. 宗教與中國民族主義--民初知識份子反教思想的學理基礎．In: Sammelband der Aufsätzen: Die Geschichte des modernen China.中國現代史 論集. Bd.6. Taipei. S. 167-182.

Yen Hsien-chun 葉憲峻 1993: Bewegung der Sinisierung nach dem Zweiten 
Weltkrieg in Taiwan, am Beispiel der elementaren Bildung. 二次世界戰後初 期臺灣之中國化運動 - 以初等教育為例. Magisterarbeit beim Institut für Pädagogik an der „National Taiwan Normal University“. 國立臺灣師範大學 教育研究所碩士論文.

--1996: Die Entwicklung der elementaren Erziehung in Taiwan. 台灣初等教育之演 進. In: Hsu Nan-hao (Hrsg.) 徐南號主編 1996: Die taiwanesische Bildungsgeschichte. 台灣教育史. Taipei.台北 S. 85-132.：

Yen Jung-chung 葉榮鐘 1971: Die Geschichte der taiwanesischen Volksbewegung. 台灣民族運動史. Taipei.

Yen Shu-fang 顏淑芳 1989: Der Gedanken der Partei in der Zeitschrift „,Free China“. ”自由中國“半月刊的政黨思想. Magisterarbeit beim Institut für Politikwissenschaft an der „Chinese Culture University“. 中國文化大學政治 研究所碩士論文.

Yu Ben-fa und Hsiung Hsien-chun 喻本伐 熊賢君 1995: Die Bildungsgeschichte Chinas.中國教育史. Taipei.

Yu Sung-nian 郁松年 1976: Studie über den Antrag für das Darlehen bei der Weltbank für Erziehung. 我國申請世銀教育計畫貸款經緯. Taipei.

Yu Ying-shih 余英時 1986: Der Sinn der chinesischen Kultur in der Modernisierung anhand ihrem Wertsystem: Chinesische Kultur und modernes Leben. 從價值系 統看中國文化的現代意義:中國文化與現代生活. Taipei.

--2005: Erklärung über Funktion und Bedeutung des kaiserlichen Beamtenprüfungssystems in der chinesischen Geschichte. 試說科舉在中國歷史上的功 能與意義. In: The Twenty-First Century Review.二十一世紀隻隹月刊. Vol.89， 06.2005, S.4-18.

Yuan Zheng 袁征 2002: From Independence of Education to Participation of Education. An Important Change in Cai Yuan-pei’s Educational Thought. 從教 
育獨立到黨化教育--蔡元培教育思想的重要變化. In: Hong Kong Journal of Social Sciences. 香港社會科學學報. No.24, Winter 2002, S. 115-135.

Yuen Chin-shu 袁金書 1991: Studie über die Großtaten des Konfuzius und seiner Schüler. 孔子及其弟子事蹟考詮. Taipei.

Zurndorfer, Harriet T. 宋漢理 2006: Geschlechtsspezifizierung innerhalb des kaiserlichen Beamtenprüfungssystems. 性別化的中國科舉制度. In: Lee Thomas H.C. (Hrsg.) 李弘祺編 2006: Tradition der Erziehung in China und Ostasien Bd. 1. - Die Erziehung und das kaiserliche Beamtenprüfungssystem in China. 中國與東亞的教育傳統(一)：中國的教育與科舉. Taipei. S. 207-224.

\section{Literatur in europäischer Sprache:}

Anderson, Benedict R. 1988: Die Erfindung der Nation. Zur Karriere eines folgenreichen Konzepts. Frankfurt/M., New York: Campus (Erweiterte Neuausgabe 1996).

Bai Limin 2005: Shaping the Ideal Child: Children and their Primers in Late Imperial China. Columbia Univ Pr.

de Bary, Wm. Theodore 1989: Chu Hsi's Aim as an Educator. In: de Bary, Wm. Theodre and Caffee, Jahn W. (Hrsg.) 1989: Neo-Confucian Education: The Formative Stage. Univ. of Califormia Press. S.186-218.

Bauer, Wolfgang 2006: Geschichte der chinesischen Philosophie: Konfuzianismus, Daoismus, Buddhismus. Hrsg. von Hans van Ess. München: Beck.

Becker, Gary S. 1993: Human Capital. A Theoretical and Empirical Analysis with Special Reference to Education, 3. Auflage.

Berger, Peter 1984: „A Model of East Asian Development: The Cultural Factor in the Post-war Taiwan Experience“, In: The Economic News. Sept. 1984 S.17-23.

Bergère, Marie-Claire 1998: Sun Yat-sen. Translated from the French by Loyd, Janet. 
Stanford, Calif. Stanford University Press.

Birge, Bettine 1989: Chu Hsi and Women’s Education. In: de Bary, Wm Theodre and Caffee, Jahn W. (Hrsg.) 1989: Neo-Confucian Education: The Formative Stage. Berkeley. Cal: Univ. of Califormia Press. S.325-367.

Blankertz, Herwig 1982: Die Geschischte der Pädagogik. Von der Aufklärung bis zur Gegenwart. Wetzlar.

Bol, Peter K. 1989: Chu-His`s Redefinition of Literati Learning. In: de Bary, Wm Theodre and Caffee, Jahn W. (Hrsg.) 1989: Neo-Confucian Education: The Formative Stage. Berkeley. Cal: Univ. of Califormia Press. S.151-185.

Bürklin, Wilhelm 1994: Die vier kleinen Tiger: die pazifische Herausforderung; Hongkong, Singapur, Taiwan, Südkorea. Frankfurt/M .: Ulltein

Cai Jian-guo 1998: Tsai Yuen-pei: Gelehrter und Mittler zwischen Ost und West. Deutsch von Stichler, Hans Christian. Münster.

Chang Chung-li 1955: The Chinese gentry: studies on their role in nineteenth-century Chinese society. Seattle: University of Washington Press.

Chun Allen 1996: From Nationalism to Natonalizing: Cultural Imagination and State Formation in Postwar Taiwan. In: Unger, Johathan 1996: Chinese Nationalism. S.126-147.

Dahrendorf, Ralf 1968: Bildung ist Bürgerrecht. Plädoyer für eine aktive Bildungspolitik. Hamburg: Wegner.

Denison, E. F. (1962). "Education, Economic Growth and Gaps in Information", Journal of Political Economy, Vol. 70, No. 5, pp.124-128.

Dirlik, Arif 1975: „, The Ideological Foundation of the New Life Movement: A Study in Couter Revolution, ,Journal of Asian Studies 34:4.

Domes, Jürgen 1969: Vertragte Revolution. Die Politik der Kuomintang in China 1923-1937, Berlin: De Gryter. 
--1970: Die Koumintang-Herrschaft in China. Hannover: Niedersächs. Landeszentrale für Polit. Bildung.

Dörnhaus, Udo 1988: Berufsbildungspolitik Taiwans im Verlaufe der wirtschaftlichen Entwicklung 1949-1985. Hamburg.

Eastman, Lloyd E. 1974: The Abortive Revolution. China under Nationalist Rule 1927-1937. Cambridge, Mass.

--1976: The Kuomingdang in the 1930s'“" in the Limits of Change: Essays on Conservative Alternatives in Republic China (Cambrige: Harvard University Press).

Eisenstadt, Shmuel N. 200: Die Vielfalt der Moderne: Ein Blick zurück auf die ersten Überlegungen zu den „multiple modernities“. Weilerswist.

Elman, Benjamin A 2000: A cultural history of civil examinations in late imperial China. Berkeley: Univ. of California Press.

---2005: On their own terms: science in China, 1550-1990. Cambridge, Mass.: Harvard Univ. Press.

--2006: A cultural history of modern science in China. Cambridge, Mass: Harvard Univ. Press.

Elman, Benjamin A. und Woodside, Alexander 1994: Education and society in late imperial China 1600-1900. Berkeley: Univ. of California Press.

Esherick, Joseph W. 1987: The Origins of the Boxer Uprising. Berkeley: University of California Press.

Freudenberg, Michael 1985: Die Frauenbewegung in China am Ende der Qing-Dynastie. Bochum.

Fei, John C.H. and Liu Tsúi-jung 1982: The Growth and Decline of Chinese Famliy Clans“. In: Journal of Interdisciplinary History. Vol.XII, No.3. S.375-408.

Franke, Wolfgang1957: Chinas kulturelle Revolution- Die Bewegung des 4. Mai 1919. 
München.

--1967: Anpassungsprobleme im chinesischen Erziehungswesen des 19. und frühen 20. Jahrhunderts. Vortrag, geh. am 13.10.1967 auf der 27. ; Versammlung deutscher Historiker in Freiburg. Hamburg

Fujikawa, Nobuo 2006: Zur Verbindung von eugenischem Gedanken und militärischem Zweck in der japanischen Pädagogik in der Zeit vor und währen des Krieges- am Bespiel der Begabungsförderungserziehung in der Kriegszeit. In: Horn, Klaus-Peter/ Ogaswara, Michio u.a.(Hrsg.) 2006:Pädagogik im Militarismus und im Nationalsozialismus. Japan und Deutschland im Vergleich. Bad Heilbrunn. S.45-66.

Furuya, Keiji 1981: Chiang Kai-shek. His Life and Times. Abridged Engl. ed. / by Chang Chun-ming. New York: St. John’s Univ.

Gälli, Anton 1988: Taiwan R.O.C.: eine chinesische Herausforderung: von der Handelsmacht zum Technologiezentrum. München: weltforum-Verl.

Geißler, Rainer 1996: Bildungsexpansion und Wandel der Bildungschancen, in: Die Sozialstruktur Deutschlands. Zur gesellschaftlichen Entwicklung mit einer Zwischenbilanz zur Vereinigung. Opladen.

--2000: Bildungsexpansion und Bildungschancen. Informationen zur politischen Bildung: Sozialer Wandel in Deutschland, Nr. 269/2000, Hersg. Bundeszentrale für polit. Bildung.

Gernet, Jacques 1988: Die chinesische Welt: Die Geschichte Chinas von den Anfängen bis zur Jetztzeit. 1. Aufl. Frankfurt a.M.

Greene, J. Megan 2000: The Greening of Taiwan’s Scientific Desert: Science and the State in the Republic of China, 1949-1969. In: Harvard Studies on Taiwan: Vol.3. S.67-94.

--2007:Taiwan's knowledge-based economy. A historical perspective on higher 
education, manpower planning and economic development. In: Asch, Robert and Greene, J. Megan (ed.) 2007: Taiwan in the 21st Century. Aspects and Limitations of a Development Model. New York.

Harbison, Frederick, and Charles A. Myers, eds.1964: Education, Manpower, and Economic Growth: Strategies of Human Resource Development. New York: McGraw-Hill.

Heinzig, Dieter 1978: Sowjetische Militärberater bei der Kuomintang 1923-1927. Baden-Baden.

Helmick, John 1972: University Entrance Examination Procedures. A Report to the Minister of Education of the Republic of China.

Heman, Friedrich 1909: Geschichte der neueren Pädagogik. Eine Darstellung der Bildungsideale der Deutschen seit der Renaissance und Reformation. Zum Unterricht für Lehrerseminare und zum Selbstudium. Zweite verbesserte und vermehrte Auflage. Osterwieck/Harz und Leipzig.

Herrmann, Ulrich 1993: Abschied vom Erziehungsstaat? Einführung in den Themenschwerpunkt. In: Zeitschrift für Pädagogik, 39. Jg. 1993, H.4., S.563-565.

--1993: Erziehungsstaat - Staatserziehung - Nationalbildung. Staatliche und gesellschaftliche Funktionen und Leistungen von Erziehung und Unterricht im Übergang vom Untertanen des Staates zur modernen Staatsbürger Gesellschaft. In: Zeitschrift für Pädagogik, 39. Jg. 1993, H.4., S.567-582.

Herrmann-Pillath, Carsten 1998: Wettbewerb der Systeme und wirtschaftliche Entwicklung im chinesischen Kulturraum. In: Herrmann-Pillath/Lackner (Hrsg.) 1998: Länderbericht China. Köln. S.261-277.

Hickey, Dennis Van Vranken 1994: United States-Taiwan Security Ties. From Cold War to beyond Containment. 
Ho Ping-ti 1959: “Aspects of Social Mobility in Imperal China.” In: Comparative Studies in Socociety and History, Vol.1. S.330-359.

--1965: The Ladder of Success in Imperial China (New York: Columbia University Press.

Hoffmann, Rainer 1980: Der Untergang des konfuzianischen China. Von Mandschureich zur Volksrepublik. Wiesbaden.

Horio Teruhisa 1998: Tennostaat und Nationalerziehung: Das kaiserliche Japan - Ein Erziehungsstaat? In: Benner, Dietrich/Schriewer, Jürgen/Tenorth, Heinz-Emar (Hrsg.): Erziehungsstaaten. Weinheim. S.305-322.

Hsiao Chih-bang 2006: Lehrerbildung als Politikum - ihre Befreiungs- und Professionalitätsprozesse in Taiwan, eine historische Darstellung - untersuchte den Wandelungsprozess der Lehrerbildung von der konfuzianischen Tradition zur modernen Professionalausbildung.

Hsu Chi-wei Stephan 1969: Die Pädagogik von Tsai Yuan-pei : die Begegnung zwischen der europäischen und chinesischen Kultur im Bereich der Bildung und Erziehung. Münster, Univ., Diss.

Huang Huang-ping and Chiu Lian-Hwang 1991: Moral and Civic Education. In: Douglas C. Smith (ed.) 1991: The Confucian Continuum: Educational Modernization in Taiwan. New York: Praeger.

Hwang Kwang-kuo1986: „Dao and Transformative Power of Confucianism: A Theory of East Asian Modernization", Alumni Paper Series 8. Honolulu: East-West Center.

Jocoby, Neil H. 1966: U.S. Aid to Taiwan. Study of Foreign Aid, Self-Help and Development. New York: Fredrick A. Praeger, Inc.

Keller, M. Theresa 1989: Back to basics: Chu Hsis elementary Learning. In: de Bary, Wm Theodre and Caffee, Jahn W. 1989: Neo-Confucian Education: The 266 
Formative Stage. University of California Press. S.219-251.

Kindermann, Gottfried-Karl (Hrsg.) 1963: Konfuzianismus, Sunyatsenismus und chinesischer Kommunismus. Dokumente zur Begründung und Selbstdarstellung des chinesischen Nationalismus. Breisgau.

--1974: Einleitung zu „Stimmen des anderen China“ Taiwan: Die Herausforderung einer chinesischen Alternative. - Zur Problemstruktur und Selbstdeutung des „anderen China“ In: Kindermann, Gottfried K. (Hrsg.) 1974: Stimmen des anderen China: Nationalchinesische Experten $\mathrm{zu}$ entwicklungspolitischen Alternativen für China. München: Weltforum Verl. S.7-23.

--1977: Pekings chinesische Gegenspieler. Theorie und Praxis nationalchinesischen Widerstandes auf Taiwan. Düsseldorf

--1980 (a): Zur Entstehungsgeschichte und Frühphase des Bürgerkrieges in China 1927-1949. In: Kindermann, Gottfried-Karl (Hrsg.) 1980: Chinas unbeendeter Bürgerkrieg. Im Spannungsfeld Peking-Taiwan-USA 1949-1980. München. S. $25-40$.

--1980 (b): Amerikas Chinapolitik im Spannungsfeld des innerchinesischen Systemkonflikts. In: Kindermann, Gottfried-Karl (Hrsg.) 1980: Chinas unbeendeter Bürgerkrieg. Im Spannungsfeld Peking- Taiwan -USA 1949-1980. Wien/München. S.41-76.

Kleinschmidt, Harald 2007: Das europäische Völkerrecht und die ungleichen Verträge um die Mitte des 19. Jahrhunderts. München.

Klintworth, Gary 1995: New Taiwan, New China: Taiwan's changing role in the AsiaPacific region. NewYork.

Kracke, Edward A.1947: Family vs. Merit in Chinese Civil Service Examination under the Empire. In: Harvard Journal of Asiatic Studies. Vol. 10, S.103-123.

--1957: Region, Family and Individual in the Chinese Examination. In: Fairbank, John 
K. (Hrsg.) Chinese Thought and Institutions Chicago: University of Chicago Press. S.251-268.

Lan Yu-su 1996: Zur aktuellen Methodik der Moralerziehung in Taiwan. Der Sinn und die Problematik der Realisierung des „Werterziehungsansatzes“ in den Lehrbuchreihen des Faches „Civics“ für den Primar-Bereich und die Anwendungsmöglichkeit dieses Modells für den Sekundar-Bereich. Dissertation an der Universität Köln.

Lee Eun-jeung 2003: „Anti-Europa“. Die Geschichte der Rezeption des Konfuzianismus und der konfuzianischen Gesellschaft seit der frühen Aufklärung; eine ideengeschichtliche Untersuchung unter besonderer Berücksichtigung der deutschen Entwicklung. Münster.

Lee Thomas H.C. 1989: Sung Schools and Education Before Chu His. In: de Bary, Wm Theodre and Caffee, Jahn W. 1989: Neo-Confucian Education: The Formative Stage. University of California Press. S.105-138.

--2000: Education in Traditional China: a history. Handbuch der Orientalistik. Vierte Abteilung; China Handbuch der Orientalistik / Hrsg. von B. Spuler. Unter Mitarb. von H. Franke ... ; Abt. 4: China / Hrsg. von H. Franke Bd. 13.

Li Kwoh-ting and Tzong-shian Yu (eds. )1982: Experiences and Lessons of Economic Development in Taiwan, Taipei: Academia Sinica.

Linebarger, Paul Myron Anthony 1957: The Political Doctrines of Sun Yat-Sen. An Exposition of San Min Chu I. Baltimore.

Liu Hui-ru 1993:,,Die 4-Mai-Bewegung aus heutiger Sicht“. In: Pohl, Karl-Heinz; Wacker, Gudrun und Liu Hui-ru (Hrsg.) 1993: Chinesische Intellektuelle im 20. Jahrhundert: Zwischen Tradition und Modernen. Hamburg. S.37-55.

Liou Wei-chih 2006: „Aus deutschem Geistesleben...“: Zur Rezeption der deutschen Pädagogik in China und Taiwan zwischen 1900 und 1960. Leipzig 
Liuo Wen-ji 1994: Rückblick und Reflexion über die Entwicklung der Hochschule seit 40 Jahren. In: Jiao Ou-yang und Huang Zhen-jie (Hrsg.) 1994: Das Ideal der Universität.

Maier, Harry 1994: Bildungsökonomie. Die Interdependenz von Bildungs- und Beschäftigungssystem.

Makeham, John 2008: Lost Soul. „Confucianism” in Contemporary Chinese Academic Discourse. Ruxue: Daotong versus Zhengtong. S.192-207.

Man-Cheong, Iona 2004: The Class of 1761 Examinations, State and Elite in Eighteenth Century China (Standford, CA.)

Martin, Bernd 1987: Japans Weg in die Moderne und das deutsche Vorbild: Historische Gemeinsamkeit zweier „,verspäteter Nationen“ (1860-1960) In: Ders. (Hrsg.) 1987: Japans Weg in die Moderne. Ein Sonderweg nach deutschem Vorbild? Frankfurt/Main, New York. S.17-44.

McBeath, Gerald A. 1998: Wealth and Freedom. Taiwan’s New Political Economy. Hampshire: Ashgate.

McKnight, Brian E. 1971: Village and Bureaucracy in Southern Sung China. Chicago \& London, The University of Chicago Press.

Meißner, Werner 1993: Intellektuelle im Zwiespalt. Fallstudien über Chang Chün-mai, Hu Shih und Ch'en Tu-hsiu. In: Pohl, Karl-Heinz; Wacker, Gudrun und Liu Hui-ru (Hrsg.) 1993: Chinesische Intellektuelle im 20. Jahrhundert: Zwischen Tradition und Modernen. Hamburg. S.125-143.

--1994: China zwischen nationalem „Sonderweg“ und universaler Modernisierung. Zur Rezeption westlichen Denkens in China. München.

Meyer, John W. 1977: The Effects of Education as an Institution. In: American Journal of sociology. Vol.83 (1), S.53-77.

Meyer, John W. und Ramirez, Francisco O.; Rubinson, Richard; Boli-Bennett, John 
1977: The World Educational Revolution, 1950-1970. In: Sociology of Education 1977 vol. 50 (October) S.242-258.

Meyer, John W.; Ramirez, Francisco O. und Conrad W. Snyder 1992: „World Expansion of Mass Education (1870-1970)“ In: Sociology of Education 65, S.128-149.

Miyazaki, Ichisada 1981: China's Examination Hell. The Civil Service Examinations of Imperial China. Translated by Conrad Schirokauer. New Haven: Yale Univ. Press.

Müller-Hofstede, Christoph 1998: Reich und mächtig- Chinas Zukunft als Nation- Ein Essay. In: Herrmann-Pillath/Lackner (Hrsg.)1998: Länderbericht China. Köln. S.534- 566.

Neugebauer, Ernst 1971: Anfänge pädagogischer Entwicklungshilfe unter dem Völkerbund in China: 1931 bis 1935. Hamburg: Institut für Asienkunde.

Noyes, W.A. Jr. 1958: Science and Diplomacy. In: ebd.: Science and Foreign Policy. New York: Foreign Policy Association.

OECD 2007: Bildung auf einen Blick. OECD-Indikatoren 2007, OECD Paris.

Osterhammel, Jürgen 1989 : China und die Weltgesellschaft vom 18. Jahrhundert bis in unsere Zeit. München.

Osterhammel, Jürgen und Petersson, Niels P. 2003: Geschichte der Globalisierung. München.

Paul, Gregor 2001: Konfuzius, Herder, Freiburg/B.

Peng Pon-to (Hrsg.): Taiwan Handbuch. Die Republik China. München.

Picht, Georg1965: Die deutsche Bildungskatastrophe. Analyse und Dokumentation. Freiburg i.Br. 1964, 2. Auflage München.

Polianski, Igor J. und Schwartz, Matthias (Hrsg.) 2009: Die Spur des Sputniks. Kulturhistorische Expeditionen ins kosmische Zeitalter. Frankfurt am Main: 
Campus-Verl.

Rist, Gilbert 1997: The History of Development. From Western Oringins to Global Faith. London und New York: Zed Books.

Robinsohn, Saul B. und Thomas, Helga 1971: Deutscher Bildungsrat Gutachten und Studien der Bildungskommission 3.: Differenzierung im SekundarschulwesenVorschläge zur Struktur der weiterführenden Schulen im Licht internationaler Erfahrungen. Stuttgart.

Roetz, Heiner 1995: Konfuzius. Beck, München.

Roger, B. Jeans, Jr. 1997: Democracy and Socialism in Republican China: The Politics of Zhang Junmai (Carsun Chang), 1906-1941, Lanham (Maryland) and Oxford (UK): Rowman \& Littlefield.

Ruth Hayhoe (Hrsg.) 1992:„Education and Modernization: The Chinese Experience“. Oxford: Pergamon Press.

--1996: China’s Universities 1895-1995. A Century of Cultural Conflict. New York \& London: Garland.

Schlomann, Friedrich Wilhelm und Friedlingstein Paulette 1976: Tschiang Kai-schek. Ein Leben für China; Bergisch Gladbach: Bastei Lübbe.

Schubert, Günter 1998: Taiwan seit 1945: Von der Entwicklungsdiktatur zur entwickelten Demokratie. In Herrmann-Pillath/Lackner (Hrsg.)1998: Länderbericht China. Köln. S.206-221.

Schubert, Volker 1998: Japan in der Meiji-Zeit-Schule im Prozess der Formierung des „modernen“ Alltagslebens. In: ebd., S.323-337.

Schultz, Theodor W. 1961: Investment in Human Capital, in: American Economic Review, 51 (1961) Vol. I. S.1-17.

Schulte, Barbara 2008: Zur Rettung des Landes“. Bildung und Beruf im China der Republikzeit. Frankfurt. 
Seiwert, Hubert 1985: Volksreligion und national Tradition in Tiawan: Studien zur regionalen Religionsgeschichte. Chines. Provinz. Stuttgart, Steiner.

Smith, Douglas C. 1991: Secondary Academic Education. In: Smith, Douglas C. (Hrsg.) 1991: The Confucian continuum: educational modernization in Taiwan. New York : Praeger.

Song Xue-ming 1998: Der chinesische Wirtschaftsraum als Faktor in der Weltwirtschaft. In: Herrmann-Pillath/Lackner (Hrsg.) 1998: Länderbericht China. S.302-324.

Spencer, Jonathan D.1995: Chinas Weg in die Moderne. München.

Strauss, Julia C. 1998: Strong Institutions in Weak Polities: State Building in Republican China, 1927-1940 (New York: Oxford University Press.

Statistisches Bundesamt Wiesbaden (Hrsg.) 1991: Länderbericht: Taiwan 1991. Stuttgart.

Statistisches Bundesamt Wiesbaden (Hrsg.) 1988: Länderbericht Taiwan 1988. Stuttgart.

Teng Ssu Yu 1971: The Taiping Rebellion and the Western Powers. Oxford.

Tenoth, Heinz - Elmar 2000: Geschichte der Erziehung. Einführung in die Grundzüge ihrer neuzeitlichen Entwicklung. Weinheim und München.

Thomson, Charles A. und Laves, Walter H.C. 1963: Culture Relations and U.S. foreign Policy Bloomington, Indiana Univ. Press.

Thomson, James C., Jr., While China Faced West - American Reformers in Nationalist China, 1928-1937. Cambridge, Massachusetts, Harvard University Press, 1969.

Tsurumi, E. Patricia 1977: Japanese colonial Education in Taiwan, 1895-1945, Harvard University Press Cambridge, Massachusetts and London, England.

Tu Wei-ming 1985: „Confucian Thought: Selfhood as Creative Transformation », 272 
State University of New York Press, Albany.

Tucker, Nancy Bernkopf 1994: Taiwan, Hong Kong and the United States, 1945-1992:

Uncertain Friedships New York: Twayne Publishers. Twayne's international history series. [no. 14]

Twitchett, Denis Crispin 1965: A Critique of some Recent studies of Modern Chinese Social-Economic History. In: Transactions of the International Conference of Orientalises in Japan, Vol. 10, S.28-41.

--1976: The birth of the Chinese meritocracy. Bureaucrats and examinations in Tang China. London.

Verlag für fremdsprachige Literatur, Peking 1978: Die Reformbewegung von 1898. In: „Serie der Geschichte des modernen China“.

Wachman, Alan M.1994: "Competing Identities in Taiwan". In: Murray A. Rubinstein (ed.) The Other Taiwan: 1945 to the Present. (Armonk, N.Y.: M.E. Sharpe.

Walton, Linda 1989: The Institutional Context of Neo-Confucianism: Scholar, Schools and Shu-yüan in Sung-Yüan China. In: de Bary, Wm Theodre and Caffee, John W. 1989: Neo-Confucian Education: The Formative Stage. S.457-492.

Wang Pei-li 1996:Wilhelm von Humboldt und Tsai Yuen-pei: eine vergleichende Analyse zweier klassischer Bildungskonzepte in der deutschen Aufklärung und in der ersten chinesischen Republik. Münster: Waxmann.

Wang Yi-chu 1966: Chinese intellectuals and the West 1872-1949. Chapel Hill, NC: Univ. of North Carolina Press.

Weber, Max 1991: Die Wirtschaftsethik der Weltreligionen. Konfuzianismus und Taoismus. Schriften 1915-1920. Herausgeben von Hewig Schmidt-Glintzer. Tübingen. 
Weggel, Oskar 1991: die Geschichte Taiwans: vom 17. Jahrhundert bis heute. Köln.

Whittome, Günter1991: Taiwan 1947. Der Aufstand gegen die Koumintang, Institut für Asienkunde, Hamburg.

Wilson, Richard 1970:Lerning to be Chinese: the political Socialization of Children in Taiwan (Cambrige, Ma.: MIT Press

Witting, Horst E. 1976: Pädagogik und Bildungspolitik Japans. Quellentexte und Dokumente. München/Basel S.89f.

Wu Pei-yi 1989: Education of Children in the Sung. In: de Bary, Wm Theodre and Caffee, Jahn W. 1989: Neo-Confucian Education: The Formative Stage.

Yang Ming-che 1974: Grundfragen der nationalchinesischen Verteidigungspolitik auf Taiwan. In: Kindermann, Gottfried-Karl (Hrsg.) 1974: Stimme des anderen China. Nationalchinesische Experten zu entwicklungspolitischen Alternativen für China. München. S.65-74.

Yen-Scharpf, Sophia 1980: Chinas Taiwan-Problem bis 1949. Die regionale Dimension. In: Kindermann, Gottfried-Karl (Hrsg.) 1980: Chinas unbeendeter Bürgerkrieg. Im Spannungsfeld Peking-Taiwan-USA (1949-1980). Wien/München. S.93-133.

You Jong-Il 1998: Income Distribution and Growth in East Ausa, The Journal of Develpment Studies, Vol. 34, No.6, S.37-65.

Yun Young-Min 1994: Class Structure and Class Mobility in East Asia: A Comparison among South Korea, Japan and Taiwan, Korea Journal of Population and Development, vol. 23, No. 2:257-281.

Zürcher, Erik 1989: Buddhism and Education in Tang Times. In: de Bary, Wm Theodre and Caffee, Jahn W. 1989: Neo-Confucian Education: The Formative Stage. University of California Press. S.19-56.

\section{Zeitung:}


1981-06-02/ United Daily News (聯合報) /P-03/

Hu Shih 胡適 1958: Rede des Amtsantritt als Prasident „Sinica Academia“ von $\mathrm{Hu}$ Shih. 中研院長胡適就職. In der Zeitungsartikel in „Central daily newspaper“ am 11. April 1958. S.1.（1958, 4 月 11 日）：中央日報, 1 版.

\section{Zeitschrift}

„Die Welt“ 天下 1996: Die Erziehung in Taiwan, Sonderband, 台灣教育特刊. Nov. 1996. Taipei.

Chinatimes Weekly 時報週刊 2005: Interview mit Tu Cheng-sheng 杜正勝專訪. am 11.07.2005, 


\begin{tabular}{|c|c|c|c|c|c|}
\hline \multicolumn{6}{|c|}{$\begin{array}{c}\text { Tabelle 1 } \\
\text { Entwicklung der Anzahl der Schulen, Schüler und der Schulbesuchsrate in den } \\
\text { Konfuzianischen Schulen und der Anzahl der Schulen sowie der Einschulungsrate in den } \\
\text { „Öffentlichen Schulen“von } 1899 \text { bis } 1944 \\
\end{array}$} \\
\hline & \multicolumn{2}{|c|}{ Öffentliche Schule } & \multicolumn{3}{|c|}{ Konfuzianische Schule } \\
\hline Jahrgang & $\begin{array}{l}\text { Anzahl der } \\
\text { Schulen }\end{array}$ & $\begin{array}{c}\text { Einschulungsrate } \\
\text { in: } \%\end{array}$ & $\begin{array}{l}\text { Anzahl der } \\
\text { Schulen }\end{array}$ & $\begin{array}{l}\text { Anzahl der } \\
\text { Schüler }\end{array}$ & $\begin{array}{c}\text { Einschulungsrate } \\
\text { in: } \%\end{array}$ \\
\hline 1899 & 96 & & 1.421 & 25.341 & 8,27 \\
\hline 1900 & 117 & & 1.473 & 26.186 & 8,43 \\
\hline 1901 & 121 & & 1.554 & 28.064 & 8,91 \\
\hline 1902 & 139 & & 1.623 & 29.742 & 9,34 \\
\hline 1903 & 146 & & 1.365 & 25.710 & 7,95 \\
\hline 1904 & 152 & & 1.080 & 21.661 & 6,58 \\
\hline 1905 & 180 & & 1.055 & 19.255 & 5,77 \\
\hline 1906 & 195 & & 914 & 19.915 & 5,87 \\
\hline 1907 & 207 & & 873 & 18.612 & 5,40 \\
\hline 1908 & 219 & & 630 & 14.782 & 4,24 \\
\hline 1909 & 231 & & 655 & 17.101 & 4,83 \\
\hline 1910 & 240 & & 567 & 15.811 & 4,39 \\
\hline 1911 & 253 & & 548 & 15.759 & 4,33 \\
\hline 1912 & 265 & & 541 & 16.302 & 4,40 \\
\hline 1913 & 280 & & 576 & 17.284 & 4,62 \\
\hline 1914 & 292 & & 638 & 19.257 & 5,11 \\
\hline 1915 & 307 & & 599 & 18.000 & 4,71 \\
\hline 1916 & 328 & & 584 & 19.320 & 4,96 \\
\hline 1917 & 352 & 13,14 & 533 & 17.641 & 4,45 \\
\hline 1918 & 421 & 15,71 & 385 & 13.314 & 3,33 \\
\hline 1919 & 438 & 20,69 & 302 & 10.936 & 2,68 \\
\hline 1920 & 495 & 25,11 & 225 & 7.639 & 1,83 \\
\hline 1921 & 531 & 27,22 & 197 & 6.962 & 1,64 \\
\hline 1922 & 592 & 28,82 & 194 & 3.664 & 0,81 \\
\hline 1923 & 715 & 28,60 & 122 & 5.283 & 1,16 \\
\hline 1924 & 725 & 28,99 & 126 & 5.165 & 1,12 \\
\hline 1925 & 728 & 29,51 & 120 & 5.137 & 1,09 \\
\hline 1926 & 735 & 28,42 & 136 & 5.486 & 1,16 \\
\hline 1927 & 743 & 29,18 & 137 & 5.312 & 1,11 \\
\hline 1928 & 749 & 29,79 & 139 & 5.597 & 1,15 \\
\hline 1929 & 754 & 31,11 & 160 & 5.700 & 1,16 \\
\hline 1930 & 757 & 33,11 & 164 & 5.968 & 1,20 \\
\hline 1931 & 761 & 34,20 & 157 & 5.378 & 1,04 \\
\hline 1932 & 762 & 35,87 & 142 & 4.700 & 0,90 \\
\hline 1933 & 769 & 37,44 & 129 & 4.494 & 0,84 \\
\hline 1934 & 770 & 39,33 & 110 & 3.524 & 0,64 \\
\hline 1935 & 781 & 41,47 & 98 & 3.099 & 0,54 \\
\hline 1936 & 785 & 43,79 & 62 & 2.411 & 0,42 \\
\hline 1937 & 788 & 46,69 & 28 & 1.407 & 0,23 \\
\hline 1938 & 796 & 49,82 & 19 & & \\
\hline 1939 & 810 & 53,15 & 17 & & \\
\hline 1940 & 825 & 57,56 & 7 & & \\
\hline 1941 & 843 & 61,60 & & & \\
\hline 1942 & 878 & 61,56 & & & \\
\hline 1943 & 905 & 65,82 & & & \\
\hline 1944 & 944 & 71,31 & & & \\
\hline
\end{tabular}

Quelle: Die Statistikbehörde unter den Verwaltungsbehörden des Gouvernements der Provinz Taiwan. 1946 (Hrsg.) S.242 und 477 und Li Yuen-Huei 1983 S.597. 


\begin{tabular}{|c|c|c|c|c|c|c|c|c|c|c|c|c|c|}
\hline \multirow[t]{2}{*}{ Jahr } & \multicolumn{2}{|c|}{ Universität } & \multicolumn{2}{|c|}{ Fachschule } & \multicolumn{2}{|c|}{$\begin{array}{l}\text { Ausbildungsstätte } \\
\text { für Lehrerausbildung }\end{array}$} & \multicolumn{2}{|c|}{ Mittelschule } & \multicolumn{2}{|c|}{ Berufsschule } & \multicolumn{2}{|c|}{$\begin{array}{l}\text { Öffentliche } \\
\text { Schule }\end{array}$} & \multirow[t]{2}{*}{$\begin{array}{c}\text { Die relative } \\
\text { Schulbesuchrate } \\
\text { in } \% \\
\end{array}$} \\
\hline & $\begin{array}{c}\text { Anzahl } \\
\text { der } \\
\text { Schulen }\end{array}$ & $\begin{array}{l}\text { Anzahl der } \\
\text { Studenten }\end{array}$ & $\begin{array}{c}\text { Anzahl } \\
\text { der } \\
\text { Schulen } \\
\end{array}$ & $\begin{array}{c}\text { Anzahl der } \\
\text { Studenten }\end{array}$ & $\begin{array}{c}\text { Anzahl } \\
\text { der } \\
\text { Schulen } \\
\end{array}$ & $\begin{array}{l}\text { Anzahl der } \\
\text { Schüler }\end{array}$ & $\begin{array}{l}\text { Anzahl der } \\
\text { Schulen }\end{array}$ & $\begin{array}{l}\text { Anzahl der } \\
\text { Schüler }\end{array}$ & $\begin{array}{c}\text { Anzahl der } \\
\text { Schulen }\end{array}$ & $\begin{array}{l}\text { Anzahl der } \\
\text { Schüler }\end{array}$ & $\begin{array}{l}\text { Anzahl der } \\
\text { Schulen }\end{array}$ & $\begin{array}{l}\text { Anzahl der } \\
\text { Schüler }\end{array}$ & \\
\hline 1900 & & & 1 & 89 & 1 & 195 & & & & & 127 & 13.272 & \\
\hline 1905 & & & 1 & 140 & 1 & 242 & 1 & 136 & & & 204 & 31.221 & \\
\hline 1910 & & & 1 & 194 & 1 & 443 & 1 & 266 & & & 291 & 49.556 & \\
\hline 1915 & & & 1 & 205 & 1 & 764 & 3 & 1.357 & 1 & 168 & 420 & 81.979 & 13,1 \\
\hline 1920 & & & 3 & 508 & 2 & 1.188 & 6 & 2.279 & 5 & 857 & 625 & 175.596 & 25,1 \\
\hline 1925 & & & 3 & 723 & 3 & 1.699 & 21 & 7.569 & 22 & 2.246 & 861 & 244.902 & 29,5 \\
\hline 1930 & 1 & 180 & 3 & 831 & 4 & 1.190 & 23 & 10.507 & 38 & 4.323 & 891 & 282.641 & 33,1 \\
\hline 1935 & 1 & 114 & 3 & 976 & 4 & 1.379 & 24 & 12.241 & 46 & 5.552 & 917 & 407.449 & 41,5 \\
\hline 1940 & 1 & 322 & 3 & 1.078 & 6 & 2.002 & 36 & 18.684 & 89 & 16.240 & 974 & 671.059 & 57,6 \\
\hline 1943 & 1 & 357 & 3 & 1.817 & 3 & 2.888 & 45 & 29.540 & 117 & 32.718 & 1099 & 932.525 & 71,3 \\
\hline
\end{tabular}

Quelle: Statistikbehörde unter den Verwaltungsbehörden des Gouvernements der Provinz Taiwan. 1946 (Hrsg.) S.1211-1212, 241-1242 


\begin{tabular}{|c|c|c|c|c|c|c|}
\hline \multicolumn{7}{|c|}{$\begin{array}{c}\text { Tabelle } 3 \\
\text { Anzahl der Schulen und der Schüler (Anzahl der gesamten Schüler, der taiwanesischen Schüler und der japanischen Schüler) und Anteil der taiwanesischen } \\
\text { Schüler in den allen Bildungseinrichtungen im Jahr 1944 }\end{array}$} \\
\hline & & $\begin{array}{l}\text { Anzahl der } \\
\text { Schulen }\end{array}$ & $\begin{array}{c}\text { Anzahl der } \\
\text { gesamten Schüler }\end{array}$ & $\begin{array}{l}\text { Anzahl der } \\
\text { Taiwanesen }\end{array}$ & $\begin{array}{l}\text { Anzahl der } \\
\text { Japaner }\end{array}$ & $\begin{array}{l}\text { Anteil der Taiwanesen } \\
\text { In:\% }\end{array}$ \\
\hline \multicolumn{2}{|c|}{$\begin{array}{c}\text { Volksschulen } \\
\text { und Grundschulen }\end{array}$} & 1.074 & 862.674 & 739.856 & 122.818 & 85,76 \\
\hline \multirow{2}{*}{$\begin{array}{l}\text { Allgemeine } \\
\text { Bildung }\end{array}$} & $\begin{array}{l}\text { Mittelschulen } \\
\text { (männlich) }\end{array}$ & 22 & 14.523 & 7.343 & 7.180 & 50,56 \\
\hline & $\begin{array}{l}\text { Mittelschulen } \\
\text { (weiblich) }\end{array}$ & 23 & 11.902 & 4.340 & 7.562 & 36,46 \\
\hline \multirow[b]{2}{*}{$\begin{array}{l}\text { Berufliche } \\
\text { Ausbildung }\end{array}$} & Berufsschulen & 27 & 13.042 & 7.876 & 5.166 & 60,39 \\
\hline & $\begin{array}{c}\text { Ausbildungseinrich } \\
\text { tungen } \\
\text { für kurzzeitige } \\
\text { Förderkurse }\end{array}$ & 90 & 7.718 & 5531 & 2.187 & 71,66 \\
\hline \multicolumn{2}{|c|}{ Lehrerausbildungsstätte } & 3 & 2.642 & 347 & 2.295 & 13,13 \\
\hline \multicolumn{2}{|c|}{ Fachhochschulen } & 3 & 1.755 & 301 & 1.454 & 17,15 \\
\hline \multicolumn{2}{|c|}{$\begin{array}{c}\text { Höhere } \\
\text { allgemeinbildende Schule }\end{array}$} & 1 & 635 & 101 & 534 & 15,91 \\
\hline \multicolumn{2}{|c|}{ Studienkolleg } & 1 & 491 & 54 & 437 & 11,00 \\
\hline \multicolumn{2}{|c|}{ Universität } & 1 & 454 & 69 & 385 & 15,20 \\
\hline
\end{tabular}

Quelle: Statistikbehörde unter den Verwaltungsbehörden des Gouvernements der Provinz Taiwan (Hrsg.) 1946 S.242. 
Tabelle 4

Entwicklung der Anzahl von Schulen, Klassen, Lehrern und Schülern sowie der relativen Schulbesuchsrate in der Grundschule von 1944-1949

\begin{tabular}{|c|c|c|c|c|c|}
\hline Jahr & Schulen & Klassen & Lehrer & Schüler & $\begin{array}{c}\text { relative } \\
\text { Schulbesuchsrate in:\% }\end{array}$ \\
\hline 1944 & 1.097 & 13.521 & 15.483 & 898.424 & 71,31 \\
\hline 1945 & 1.053 & 13.656 & 14.914 & 850.097 & 80,01 \\
\hline 1946 & 1.130 & 13.683 & 15.355 & 823.400 & 78,56 \\
\hline 1947 & 1.160 & 14.072 & 16.083 & 855.821 & 79,02 \\
\hline 1948 & 1.185 & 14.519 & 17.369 & 840.783 & 77,14 \\
\hline 1949 & 1.199 & 15.539 & 19.000 & 892.758 & 79,07 \\
\hline
\end{tabular}

Quelle: Bildungsbehörde unter den Verwaltungsbehörden des Gouvernements der Provinz Taiwan. (Hrsg.) 1955 S.291f.

Tabelle 5

Entwicklung der Schülerzahlen in Junior High Schools, Junior Vocational High Schools, dreijährigen Lehrerausbildungsstätten, Senior High Schools und Senior Vocational High Schools, fünfjährigen pädagogischen Fachschulen, Fachschulen und Universitäten von 1945 bis 1949

\begin{tabular}{|c|c|c|c|c|c|c|c|c|}
\hline Jahr & $\begin{array}{c}\text { Junior High } \\
\text { Schools }\end{array}$ & $\begin{array}{c}\text { Junior } \\
\text { Vocational } \\
\text { Schools }\end{array}$ & $\begin{array}{c}\text { dreijährige } \\
\text { Lehrerausbild } \\
\text { ungsstätten }\end{array}$ & $\begin{array}{c}\text { Senior } \\
\text { High } \\
\text { Schools }\end{array}$ & $\begin{array}{c}\text { Senior } \\
\text { Vocational } \\
\text { Schools }\end{array}$ & $\begin{array}{c}\text { fünfjährigen } \\
\text { Pädagogischen } \\
\text { Fachschulen }\end{array}$ & Fachschulen & Universitäten \\
\hline 1945 & 36.088 & 21.535 & 581 & 4.075 & 2.444 & & & \\
\hline 1946 & 36.222 & 20.773 & 537 & 4.503 & 2.543 & 2.458 & & 2.983 \\
\hline 1947 & 46.955 & 22.931 & 830 & 6.519 & 4.721 & 2.736 & & 3.176 \\
\hline 1948 & 60.448 & 24.403 & 413 & 9.939 & 7.336 & 3.684 & 79 & 4.033 \\
\hline 1949 & 60.713 & 23.655 & 528 & 15.667 & 9.500 & 4.501 & 969 & 4.937 \\
\hline
\end{tabular}

Quelle: Bildungsbehörde der Gouvernementverwaltung der Provinz Taiwan. (Hrsg.) 1955 S.294-299 


\begin{tabular}{|c|c|c|c|c|c|c|}
\hline \multicolumn{7}{|c|}{$\begin{array}{c}\text { Tabelle } 6 \\
\text { Entwicklung der relativen Schulbesuchsrate, der Anzahl der Schulen, Schulklassen und Schüler } \\
\text { sowie der durchschnittlichen Anzahl der Klassen und Klassenstärke in der Elementarbildung } \\
\text { von 1950-1990 }\end{array}$} \\
\hline Jahr & $\begin{array}{l}\text { Die relative } \\
\text { Schulbe- } \\
\text { suchsrate } \\
\text { In \% }\end{array}$ & Schulen & Schulklassen & Schüler & $\begin{array}{l}\text { Durchschnittliche } \\
\text { Anzahl der } \\
\text { Klassen }\end{array}$ & $\begin{array}{l}\text { Durchschnittliche } \\
\text { Klassestärke }\end{array}$ \\
\hline 1950 & 79,98 & 1.231 & 16.856 & 906.950 & 13,69 & 53,81 \\
\hline 1951 & 81,49 & 1.248 & 17.743 & 970.664 & 14,22 & 54,71 \\
\hline 1952 & 84,00 & 1.251 & 18.833 & $1.003,304$ & 15,05 & 53,27 \\
\hline 1953 & 87,75 & 1.300 & 20.211 & 1.060 .324 & 15,55 & 52,46 \\
\hline 1954 & 90,83 & 1.350 & 22.025 & 1.133 .438 & 16,31 & 51,46 \\
\hline 1955 & 92,33 & 1.446 & 24.127 & 1.244 .029 & 16,69 & 51,56 \\
\hline 1956 & 93,82 & 1.537 & 26.189 & 1.344 .432 & 17,04 & 51,34 \\
\hline 1957 & 94,61 & 1.597 & 28.307 & 1.480 .557 & 17,73 & 5230 \\
\hline 1958 & 94,84 & 1.663 & 30.945 & 1.642 .888 & 18,61 & 53,09 \\
\hline 1959 & 95,44 & 1.757 & 33.586 & 1.777 .118 & 19,11 & 52,91 \\
\hline 1960 & 95,59 & 1.843 & 35.944 & 1.888 .783 & 19,50 & 52,55 \\
\hline 1961 & 96,00 & 1.932 & 37.906 & 1.997 .016 & 19,62 & 52,68 \\
\hline 1962 & 96,52 & 1.995 & 39.712 & 2.097.957 & 19,91 & 52,83 \\
\hline 1963 & 96,71 & 2.067 & 41.114 & 2.148 .652 & 19,89 & 52,26 \\
\hline 1964 & 96,83 & 2.107 & 42.139 & 2.202 .867 & 19,99 & 52,28 \\
\hline 1965 & 97,15 & 2.143 & 43.338 & 2.257 .720 & 20,22 & 52,10 \\
\hline 1966 & 97,16 & 2.175 & 44.382 & 2.307.955 & 20,41 & 52,00 \\
\hline 1967 & 97,52 & 2.208 & 45.171 & 2.348 .218 & 20,46 & 51,99 \\
\hline 1968 & 97,67 & 2.244 & 45.780 & 2.383 .204 & 20,40 & 52,06 \\
\hline 1969 & 97,62 & 2.275 & 47.084 & 2.428 .041 & 20,70 & 51,57 \\
\hline 1970 & 98,00 & 2.319 & 48.404 & 2.445 .405 & 20,87 & 50,52 \\
\hline 1971 & 98,02 & 2.331 & 49.300 & 2.456 .615 & 21,15 & 49,83 \\
\hline 1972 & 98,13 & 2.337 & 49.432 & 2.459 .743 & 21,15 & 49,76 \\
\hline 1973 & 98,09 & 2.349 & 49.333 & 2.431 .440 & 21,00 & 49,29 \\
\hline 1974 & 98,46 & 2.354 & 49.421 & 2.406 .531 & 20,99 & 48,69 \\
\hline 1975 & 99,29 & 2.376 & 49.322 & 2.364 .961 & 20,76 & 47,95 \\
\hline 1976 & 99,42 & 2.378 & 49.682 & 2.341 .413 & 20,89 & 47,13 \\
\hline 1977 & 99,57 & 2.400 & 50.109 & 2.319.342 & 20,88 & 46,29 \\
\hline 1978 & 99,64 & 2.412 & 50.339 & 2.278 .726 & 20,87 & 45,27 \\
\hline 1979 & 99,68 & 2.421 & 50.376 & 2.256 .363 & 20,81 & 44,79 \\
\hline 1980 & 99,72 & 2.428 & 50.345 & 2.233 .706 & 20,73 & 44,37 \\
\hline 1981 & 99,76 & 2.444 & 50.622 & 2.213 .179 & 20,71 & 43,72 \\
\hline 1982 & 99,79 & 2.457 & 50.715 & 2.226 .699 & 20,64 & 43,91 \\
\hline 1983 & 99,81 & 2.464 & 51.091 & 2.242 .641 & 20,73 & 43,90 \\
\hline 1984 & 99,82 & 2.474 & 51.718 & 2.273.390 & 20,90 & 43,96 \\
\hline
\end{tabular}




\begin{tabular}{|l|l|l|l|l|l|l|}
\hline 1985 & 99,85 & 2.486 & 52.732 & 2.321 .700 & 21,21 & 44,03 \\
\hline 1986 & 99,87 & 2.486 & 53.885 & 2.364 .438 & 21,68 & 43,88 \\
\hline 1987 & 99,89 & 2.472 & 55.070 & 2.400 .614 & 22,28 & 43,59 \\
\hline 1988 & 99,90 & 2.478 & 55.896 & 2.407 .166 & 22,56 & 43,07 \\
\hline 1989 & 99,90 & 2.484 & 56.315 & 2.384 .801 & 22,67 & 42,35 \\
\hline 1990 & 99,89 & 2.487 & 56.120 & 2.354 .113 & 22,57 & 41,95 \\
\hline
\end{tabular}

Quelle: Bildungsministerium (Hrsg.) 2008: Educational Statistics of the Republic of China 2008 S. 4, 6, 20, 22 und 34

Durchschnittliche Anzahl der Klassen und durchschnittliche Klassenstärken sind eigene Berechnung 
Tabelle 7

Entwicklung der Einschulungsrate in der Sekundarstufe I bzw. Junior High School und Junior vocational High School,

Anzahl der Schulen, Schulklassen und Schüler sowie die durchschnittliche Anzahl der Klassen pro Schule und Klassenstärke in den Junior High Schools und Anzahl der Schulen und Schüler in den Junior Vocational High Schools von 1950 bis1967

\begin{tabular}{|c|c|c|c|c|c|c|c|c|c|}
\hline \multirow[b]{2}{*}{ Jahr } & \multirow{2}{*}{$\begin{array}{c}\text { Einschulungsrate: } \\
\text { in: } \%\end{array}$} & \multicolumn{5}{|c|}{ Junior High School } & \multicolumn{3}{|c|}{ Junior vocational High School } \\
\hline & & $\begin{array}{l}\text { Anzahl der } \\
\text { Schulen }\end{array}$ & $\begin{array}{c}\text { Anzahl der } \\
\text { Klassen }\end{array}$ & $\begin{array}{l}\text { Anzahl der } \\
\text { Schüler }\end{array}$ & $\begin{array}{l}\text { Durchschnittliche } \\
\text { Anzahl der Klassen }\end{array}$ & $\begin{array}{l}\text { Durchschnittliche } \\
\text { Klassestärke }\end{array}$ & $\begin{array}{c}\text { Anzahl der } \\
\text { Schulen }\end{array}$ & $\begin{array}{l}\text { Anzahl der } \\
\text { Klassen }\end{array}$ & $\begin{array}{c}\text { Anzahl der } \\
\text { Schüler }\end{array}$ \\
\hline 1950 & 31,78 & 66 & 1.322 & 61.082 & 20,03 & 46,20 & 77 & 567 & 23.211 \\
\hline 1951 & 38,60 & 61 & 1.352 & 64.370 & 22,16 & 47,61 & 77 & 561 & 23.541 \\
\hline 1952 & 34,85 & 54 & 1.468 & 71.900 & 27,19 & 48,98 & 77 & 594 & 25.860 \\
\hline 1953 & 36,50 & 53 & 1.706 & 84.513 & 32,19 & 49,54 & 81 & 676 & 29.787 \\
\hline 1954 & 39,71 & 55 & 2.023 & 101.005 & 36,78 & 49,93 & 91 & 798 & 35.007 \\
\hline 1955 & 43,94 & 54 & 2.284 & 116.192 & 42,30 & 50,87 & 95 & 895 & 39.211 \\
\hline 1956 & 47,75 & 64 & 2.667 & 133.687 & 41,67 & 50,13 & 97 & 926 & 40.493 \\
\hline 1957 & 49,27 & 80 & 3.078 & 150.594 & 38,48 & 48,93 & 105 & 917 & 39.445 \\
\hline 1958 & 51,07 & 81 & 3.344 & 160.490 & 41,28 & 47,99 & 109 & 888 & 36.728 \\
\hline 1959 & 51,76 & 91 & 3.650 & 177.842 & 40,11 & 48,72 & 109 & 871 & 37.205 \\
\hline 1960 & 52,24 & 105 & 4.123 & 205.853 & 39,27 & 49,93 & 109 & 888 & 39.720 \\
\hline 1961 & 53,79 & 144 & 4.878 & 252.107 & 33,88 & 51,68 & 111 & 910 & 42.227 \\
\hline 1962 & 55,14 & 181 & 5.622 & 295.363 & 31,06 & 52,54 & 110 & 915 & 42.828 \\
\hline 1963 & 54,06 & 221 & 6.450 & 340.972 & 29,19 & 52,86 & 114 & 894 & 42.500 \\
\hline 1964 & 55,64 & 253 & 7.195 & 382.998 & 28,44 & 53,23 & 121 & 891 & 42.638 \\
\hline 1965 & 58,23 & 272 & 7.968 & 426.822 & 29,29 & 53,57 & 130 & 906 & 43.461 \\
\hline 1966 & 59,04 & 271 & 8.554 & 456.090 & 31,57 & 53,32 & 128 & 930 & 44.789 \\
\hline 1967 & 63,66 & 288 & 9.328 & 499.843 & 32,39 & 53,59 & 136 & 994 & 48.749 \\
\hline
\end{tabular}

Quelle: Educational Statistics of the Republic of China 2008 S.4, 6, 16, 18, 20, 22 und 35

Die durchschnittliche Anzahl der Klassen pro Schule und die durchschnittliche Klassenstärke sind eigene Berechnung. 


\begin{tabular}{|c|c|c|c|c|c|}
\hline \multicolumn{6}{|c|}{$\begin{array}{c}\text { Tabelle 7a } \\
\text { Die Schulbesuchsrate zu Grundschulen, Junior High Schools, Senior High Schools und }\end{array}$} \\
\hline $\mathrm{Jahr}$ & Schulbesuchsrate & $\begin{array}{c}\text { Absolventen } \\
\text { der } \\
\text { Grundschulen }\end{array}$ & \begin{tabular}{|} 
Absolventen \\
der \\
Junior High \\
Schools
\end{tabular} & $\begin{array}{c}\text { Absolventen } \\
\text { der Senior } \\
\text { High } \\
\text { Schools }\end{array}$ & $\begin{array}{c}\text { Absolventen } \\
\text { der Senior } \\
\text { Vocational } \\
\text { Schools }\end{array}$ \\
\hline 1950 & 79,98 & 31,78 & 51,15 & 39,76 & $\ldots$ \\
\hline 1951 & 81,49 & 38,60 & 57,26 & 39,60 & $\ldots$ \\
\hline 1952 & 84,00 & 34,85 & 56,96 & 26,27 & $\ldots$ \\
\hline 1953 & 87,75 & 36,50 & 61,04 & 41,75 & $\ldots$ \\
\hline 1954 & 90,83 & 39,71 & 64,20 & 43,01 & $\ldots$ \\
\hline 1955 & 92,33 & 43,94 & 63,13 & 44,27 & $\ldots$ \\
\hline 1956 & 93,82 & 47,75 & 71,39 & 41,94 & $\ldots$ \\
\hline 1957 & 94,61 & 49,27 & 71,44 & 37,16 & $\ldots$ \\
\hline 1958 & 94,84 & 51,07 & 71,57 & 36,85 & $\ldots$ \\
\hline 1959 & 95,44 & 51,76 & 75,98 & 37,49 & $\ldots$ \\
\hline 1960 & 95,59 & 52,24 & 75,88 & 43,41 & $\ldots$ \\
\hline 1961 & 96,00 & 53,79 & 78,60 & 44,65 & $\ldots$ \\
\hline 1962 & 96,52 & 55,14 & 76,16 & 41,83 & $\ldots$ \\
\hline 1963 & 96,71 & 54,06 & 73,32 & 45,02 & $\ldots$ \\
\hline 1964 & 96,83 & 55,64 & 76,53 & 41,07 & $\ldots$ \\
\hline 1965 & 97,15 & 58,23 & 78,52 & 38,30 & $\ldots$ \\
\hline 1966 & 97,16 & 59,04 & 75,80 & 38,62 & $\ldots$ \\
\hline 1967 & 97,52 & 63,66 & 77,97 & 40,52 & $\ldots$ \\
\hline 1968 & 97,67 & 74,17 & 83,95 & 38,95 & $\ldots$ \\
\hline 1969 & 97,62 & 75,53 & 83,15 & 39,73 & $\ldots$ \\
\hline 1970 & 98,00 & 78,59 & 82,66 & 41,92 & $\ldots$ \\
\hline 1971 & 98,02 & 80,85 & 69,62 & 43,47 & $\ldots$ \\
\hline 1972 & 98,13 & 83,31 & 71,17 & 42,62 & $\ldots$ \\
\hline 1973 & 98,09 & 83,71 & 67,90 & 37,92 & $\ldots$ \\
\hline 1974 & 98,46 & 88,03 & 67,57 & 39,68 & $\ldots$ \\
\hline 1975 & 99,29 & 89,47 & 65,82 & 39,80 & $\ldots$ \\
\hline 1976 & 99,42 & 90,41 & 61,57 & 42,39 & $\ldots$ \\
\hline 1977 & 99,57 & 93,60 & 60,04 & 43,59 & $\ldots$ \\
\hline 1978 & 99,64 & 94,08 & 60,42 & 40,43 & $\ldots$ \\
\hline 1979 & 99,68 & 95,58 & 62,75 & 43,96 & $\ldots$ \\
\hline 1980 & 99,72 & 96,14 & 65,16 & 44,64 & $\ldots$ \\
\hline 1981 & 99,76 & 96,77 & 68,11 & 45,39 & $\ldots$ \\
\hline 1982 & 99,79 & 97,96 & 71,52 & 46,03 & $\ldots$ \\
\hline 1983 & 99,81 & 97,95 & 69,55 & 46,40 & $\ldots$ \\
\hline 1984 & 99,82 & 98,70 & 70,54 & 44,36 & $\ldots$ \\
\hline 1985 & 99,85 & 98,73 & 71,31 & 40,19 & $\ldots$ \\
\hline
\end{tabular}




\begin{tabular}{|c|c|c|c|c|c|}
\hline 1986 & 99,87 & 99,04 & 77,13 & 40,98 & $\ldots$ \\
\hline 1987 & 99,89 & 99,51 & 79,32 & 46,26 & 2,83 \\
\hline 1988 & 99,90 & 99,09 & 79,51 & 45,53 & 4,57 \\
\hline 1989 & 99,90 & 99,62 & 79,60 & 44,40 & 7,00 \\
\hline 1990 & 99,89 & 99,77 & 84,70 & 48,58 & 12,92 \\
\hline
\end{tabular}

Schulbesuchsrate der Absolventen der Grundschulen = Schülerzahl der ersten Schuljahr in den Junior High School/ Anzahl der Absolventen der Grundschule*100

Quote des Besuchs zur weiteren Bildung der Absolventen der Junior High Schools = Anzahl des ersten Schuljahrs in den Senior High Schools, Senior Vocational High Schools und der fünfjährigen Fachschulen/ Anzahl der Absolventen der Junior High School*100

Quote des Besuchs zur weiteren Bildung der Absolventen der Senior High Schools = Anzahl des ersten Schuljahrs in den Fachhochschulen, Universitäten, dreijährigen Fachschule/ Anzahl der Absolventen der Senior High School*100

Quote des Besuchs zur weiteren Bildung der Absolventen der Senior Vocational High Schools $=$ Anzahl des ersten Schuljahrs in den Fachhochschulen, Universitäten, dreijährigen und der zweijährigen Fachschulen/ Anzahl der Absolventen der Senior Vocational High School*100 
Tabelle 8

Wandel der Anzahl der Schulen und Schüler an den Senior High Schools, Senior Vocational High Schools und Fachschulen von 1950 bis 1967

\begin{tabular}{|c|c|c|c|c|c|c|c|c|}
\hline \multirow{3}{*}{ Jahr } & \multicolumn{2}{|c|}{$\begin{array}{c}\text { Senior High } \\
\text { Schools }\end{array}$} & \multicolumn{4}{|c|}{ Berufsbildende Schulen } & \multirow{3}{*}{\multicolumn{2}{|c|}{$\begin{array}{l}\text { Das Verhältnis der } \\
\text { Schüler } \\
\text { zwischen Senior High } \\
\text { Schools und } \\
\text { Berufsschulen }\end{array}$}} \\
\hline & \multirow{2}{*}{$\begin{array}{l}\text { Anzahl } \\
\text { der } \\
\text { Schulen }\end{array}$} & \multirow{2}{*}{$\begin{array}{l}\text { Anzahl } \\
\text { der } \\
\text { Schüler }\end{array}$} & \multicolumn{2}{|c|}{$\begin{array}{c}\text { Senior Vocational } \\
\text { High } \\
\text { Schools } \\
\end{array}$} & \multicolumn{2}{|c|}{ Fachschulen } & & \\
\hline & & & $\begin{array}{c}\text { Anzahl der } \\
\text { Schulen }\end{array}$ & $\begin{array}{c}\text { Anzahl } \\
\text { der } \\
\text { Schüler }\end{array}$ & $\begin{array}{c}\text { Anzahl } \\
\text { der Schulen }\end{array}$ & $\begin{array}{c}\text { Anzahl } \\
\text { der Schüler }\end{array}$ & & \\
\hline 1950 & 62 & 18.866 & 77 & 11.226 & 3 & & 62,69 & 37,31 \\
\hline 1951 & 68 & 21.303 & 77 & 13.075 & 4 & & 61,97 & 38,03 \\
\hline 1952 & 75 & 21.046 & 77 & 14.232 & 4 & & 59,66 & 40,34 \\
\hline 1953 & 80 & 21.793 & 81 & 15.814 & 5 & & 57,95 & 42,05 \\
\hline 1954 & 82 & 24.198 & 91 & 17.823 & 7 & & 57,59 & 42,41 \\
\hline 1955 & 92 & 30.169 & 95 & 21.186 & 5 & & 58,75 & 41,25 \\
\hline 1956 & 105 & 37.253 & 97 & 25.410 & 6 & & 59,45 & 40,55 \\
\hline 1957 & 117 & 43.922 & 105 & 30.378 & 6 & & 59,11 & 40,89 \\
\hline 1958 & 127 & 48.394 & 109 & 35.104 & 7 & 1.425 & 57,96 & 42,04 \\
\hline 1959 & 135 & 54.314 & 109 & 40.595 & 8 & 1.729 & 57,23 & 42,77 \\
\hline 1960 & 139 & 57.512 & 109 & 44.617 & 12 & 2.115 & 56,31 & 43,69 \\
\hline 1961 & 136 & 62.548 & 111 & 46.108 & 14 & 2.148 & 57,57 & 42,43 \\
\hline 1962 & 139 & 70.950 & 110 & 48.905 & 15 & 2.173 & 59,20 & 40,80 \\
\hline 1963 & 140 & 82.892 & 114 & 54.421 & 15 & 3.237 & 60,37 & 39,63 \\
\hline 1964 & 143 & 100.873 & 121 & 64.205 & 20 & 6.387 & 61,11 & 38,89 \\
\hline 1965 & 142 & 116.197 & 130 & 74.114 & 35 & 14.384 & 61,06 & 38,94 \\
\hline 1966 & 160 & 127.490 & 128 & 83.102 & 48 & 28.215 & 60,54 & 39,46 \\
\hline 1967 & 170 & 140.604 & 136 & 94.547 & 57 & 40.372 & 59,79 & 40,21 \\
\hline
\end{tabular}

Quelle: Bildungsministerium (Hrsg.) 2008: Educational Statistics of the Republic of China 2008 S. 4, 6, 20, 22 und 34.

Es fehlte in der Statistik die Anzahl der Schüler in den ersten drei Jahre in der Fachschule 


\begin{tabular}{|c|c|c|c|c|c|c|c|c|}
\hline & & $\begin{array}{r}\text { Entwicklu } \\
\text { in den Junio }\end{array}$ & $\begin{array}{l}\text { g der Schi } \\
\text { und Senio }\end{array}$ & $\begin{array}{l}\text { Tabe } \\
\text { zahlen in } \\
\text { ocational }\end{array}$ & $\begin{array}{l}9 \\
\text { terschied } \\
\text { gh Schoo }\end{array}$ & $\begin{array}{l}\text { chen Lehrgä } \\
\text { von } 1952 \text { b }\end{array}$ & $\begin{array}{l}\text { en } \\
1967\end{array}$ & \\
\hline & $\begin{array}{c}\text { Gesamte } \\
\text { Anzahl }\end{array}$ & \begin{tabular}{|c} 
Agrarwirt- \\
schaft
\end{tabular} & Gewerbe & Handel & $\begin{array}{c}\text { Meeres } \\
\text { wesen }\end{array}$ & $\begin{array}{c}\text { Medizin } \\
\text { und Pflege }\end{array}$ & $\begin{array}{c}\text { Haus- } \\
\text { wirtschaft }\end{array}$ & Übrige \\
\hline 1952 & 40.092 & 14.735 & 8.868 & 12.877 & 1.189 & 441 & 1.982 & 0 \\
\hline 1953 & 45.601 & 17.582 & 9.258 & 14.265 & 1.450 & 498 & 2.548 & 0 \\
\hline 1954 & 52.830 & 20.604 & 10.257 & 16.185 & 1.572 & 559 & 3.653 & 0 \\
\hline 1955 & 60.397 & 23.206 & 11.279 & 18.905 & 1.724 & 592 & 4.560 & 131 \\
\hline 1956 & 65.903 & 24.208 & 12.041 & 21.910 & 1.735 & 657 & 5.068 & 284 \\
\hline 1957 & 69.823 & 23.624 & 13.137 & 25.134 & 1.559 & 690 & 5.182 & 497 \\
\hline 1958 & 71.832 & 22.792 & 12.805 & 28.259 & 1.692 & 748 & 5.182 & 354 \\
\hline 1959 & 77.800 & 22.983 & 13.182 & 32.782 & 1.846 & 880 & 5.726 & 401 \\
\hline 1960 & 84.337 & 24.765 & 13.030 & 36.612 & 1.963 & 1.083 & 6.571 & 313 \\
\hline 1961 & 88.335 & 25.350 & 13.142 & 38.941 & 2.195 & 1.103 & 7.394 & 210 \\
\hline 1962 & 91.963 & 25.948 & 13.367 & 40.758 & 2.334 & 1.199 & 7.983 & 104 \\
\hline 1963 & 96.888 & 26.163 & 13.714 & 44.542 & 2.711 & 1.336 & 8.241 & 181 \\
\hline 1964 & 106.811 & 26.914 & 15.152 & 50.988 & 3.022 & 1.496 & 8.993 & 246 \\
\hline 1965 & 117.575 & 26.776 & 17.396 & 58.555 & 3.441 & 1.936 & 9.471 & 0 \\
\hline 1966 & 127.891 & 26.791 & 19.266 & 66.130 & 3.900 & 2.201 & 9.603 & 0 \\
\hline 1967 & 143.296 & 26.844 & 24.123 & 75.178 & 4.401 & 2.936 & 9.814 & 0 \\
\hline
\end{tabular}

Quelle: CEPD 1978 (Hrsg.): Taiwan Statistical Data Book 1978 S.256.

\begin{tabular}{|c|c|c|c|c|c|c|c|c|}
\hline & \multicolumn{9}{|c|}{ Tabelle 9a } \\
\hline Jahrgang & $\begin{array}{c}\text { Entwicklung der Schüleranzahl und Anteil der Schüler in unterschiedlichen Lehrgängen in den } \\
\text { Junior und Senior Vocational High Schools in den Jahren 1952,1960 und 1967 }\end{array}$ \\
\hline $\begin{array}{c}\text { Agrarwirt- } \\
\text { Schaft }\end{array}$ & Gewerbe & Handel & $\begin{array}{c}\text { Meeres- } \\
\text { wesen }\end{array}$ & $\begin{array}{c}\text { Medizin } \\
\text { und } \\
\text { Pflege }\end{array}$ & $\begin{array}{c}\text { Haus- } \\
\text { wirtschaft }\end{array}$ & Kunst & Insgesamt \\
\hline $\begin{array}{c}\text { Anteil } \\
\text { in \% }\end{array}$ & 14.735 & 8.868 & 12.877 & 1.189 & 411 & 1.928 & & 40.092 \\
\hline 1960 & 24.765 & 13.030 & 36.612 & 1.963 & 1.083 & 6.571 & 313 & 84.337 \\
\hline $\begin{array}{c}\text { Anteil } \\
\text { in \% }\end{array}$ & 29,36 & 15,45 & 43,41 & 2,33 & 1,28 & 7,79 & 0,37 & 100 \\
\hline 1967 & 26.844 & 24.123 & 75.178 & 4.401 & 2.936 & 9.814 & 0 & 143.296 \\
\hline $\begin{array}{c}\text { Anteil } \\
\text { in \% }\end{array}$ & 18,73 & 16,83 & 52,46 & 3,07 & 2,05 & 6,85 & 0 & 100 \\
\hline
\end{tabular}

Anteil der Schüler in unterschiedlichen Lehrgängen ist eigene Berechnung 


\begin{tabular}{|c|c|c|c|c|c|c|c|c|}
\hline \multicolumn{9}{|c|}{$\begin{array}{c}\text { Tabelle } 10 \\
\text { Entwicklung der Anzahl der Fachschulen, Fachhochschulen und Universitäten } \\
\text { sowie die Studentenzahl in den Hochschulen von } 1950 \text { bis } 1990\end{array}$} \\
\hline Jahr & $\begin{array}{l}\text { Anzahl der } \\
\text { Fachschulen }\end{array}$ & $\begin{array}{l}\text { Anzahl der } \\
\text { Studenten } \\
\text { im fünfjährigen } \\
\text { Studiengang }\end{array}$ & $\begin{array}{l}\text { Anzahl der } \\
\text { Studenten } \\
\text { im dreijährigen } \\
\text { Studiengang }\end{array}$ & $\begin{array}{l}\text { Anzahl der } \\
\text { Studenten } \\
\text { im zweijährigen } \\
\text { Studiengang }\end{array}$ & $\begin{array}{l}\text { Anzahl der } \\
\text { Studenten in } \\
\text { den } \\
\text { Fachschulen }\end{array}$ & $\begin{array}{l}\text { Anzahl } \\
\text { der } \\
\text { Fachhoch- } \\
\text { schulen }\end{array}$ & $\begin{array}{c}\text { Anzahl der } \\
\text { Universitäten }\end{array}$ & $\begin{array}{l}\text { Anzahl der } \\
\text { Studeten } \\
\text { im Bachlor } \\
\text { Studium }\end{array}$ \\
\hline 1950 & 3 & & & & 1.286 & 3 & 1 & 5.374 \\
\hline 1951 & 4 & & & & 2.140 & 3 & 1 & 6.057 \\
\hline 1952 & 4 & & & & 3.171 & 3 & 1 & 6.853 \\
\hline 1953 & 5 & & & & 4.240 & 3 & 1 & 7.687 \\
\hline 1954 & 7 & & & & 4.587 & 5 & 2 & 9.001 \\
\hline 1955 & 5 & & & & 4.545 & 6 & 4 & 13.460 \\
\hline 1956 & 6 & & & & 5.025 & 5 & 6 & 17.309 \\
\hline 1957 & 6 & & & & 4.879 & 5 & 6 & 20.394 \\
\hline 1958 & 7 & 1.425 & 4.386 & & 5.822 & 7 & 7 & 21.739 \\
\hline 1959 & 8 & 1.729 & 3.895 & & 5.624 & 7 & 7 & 23.709 \\
\hline 1960 & 12 & 2.115 & 5.773 & & 7.888 & 8 & 7 & 26.735 \\
\hline 1961 & 14 & 2.148 & 6.218 & & 8.366 & 8 & 8 & 29.524 \\
\hline 1962 & 15 & 2.173 & 8.901 & & 11.074 & 9 & 9 & 32.532 \\
\hline 1963 & 15 & 3.237 & 9.702 & & 13.171 & 10 & 10 & 37.719 \\
\hline 1964 & 20 & 6.387 & 11.536 & & 17.923 & 11 & 10 & 45.203 \\
\hline 1965 & 35 & 14.384 & 15.150 & & 29.534 & 11 & 10 & 54.819 \\
\hline 1966 & 48 & 28.215 & 19.284 & & 47.499 & 11 & 10 & 65.245 \\
\hline 1967 & 57 & 40.372 & 23.039 & & 63.411 & 13 & 9 & 73.881 \\
\hline 1968 & 63 & 53.663 & 25.033 & 760 & 79.456 & 14 & 8 & 80.255 \\
\hline 1969 & 69 & 67.831 & 25.968 & 2.189 & 95.988 & 13 & 9 & 86.233 \\
\hline 1970 & 70 & 78.657 & 26.505 & 3.166 & 108.328 & 13 & 9 & 92.850 \\
\hline 1971 & 73 & 85.041 & 29.579 & 4.626 & 119.146 & 14 & 9 & 100.455 \\
\hline 1972 & 76 & 89.745 & 22.561 & 26.004 & 138.310 & 14 & 9 & 109.827 \\
\hline 1973 & 76 & 93.240 & 25.445 & 28.936 & 147.621 & 14 & 9 & 120.304 \\
\hline 1974 & 76 & 94.446 & 27.599 & 27.872 & 149.917 & 15 & 9 & 128.930 \\
\hline 1975 & 76 & 95.815 & 28.233 & 26.178 & 150.226 & 16 & 9 & 135.297 \\
\hline 1976 & 76 & 99.435 & 28.429 & 26.192 & 154.056 & 16 & 9 & 140.857 \\
\hline 1977 & 76 & 104.161 & 28.983 & 27.362 & 160.506 & 17 & 9 & 142.921 \\
\hline 1978 & 75 & 109.279 & 29.151 & 28.105 & 166.535 & 17 & 9 & 145.210 \\
\hline 1979 & 75 & 115.926 & 29.543 & 29.154 & 174.623 & 15 & 11 & 149.370 \\
\hline
\end{tabular}




\begin{tabular}{|l|l|l|l|l|l|l|l|l|}
\hline 1980 & 77 & 129.156 & 29.715 & 31.380 & 183.134 & 11 & 16 & 153.088 \\
\hline 1981 & 77 & 122.039 & 29.881 & 33.864 & 192.901 & 11 & 16 & 158.181 \\
\hline 1982 & 77 & 129.156 & 29.444 & 39.018 & 203.722 & 6 & 16 & 163.482 \\
\hline 1983 & 77 & 135.260 & 29.496 & 46.176 & 216.165 & 6 & 16 & 169.341 \\
\hline 1984 & 77 & 140.493 & 29.236 & 52.924 & 227.492 & 6 & 16 & 173.908 \\
\hline 1985 & 77 & 145.332 & 29.008 & 56.957 & 236.824 & 12 & 16 & 179.334 \\
\hline 1986 & 77 & 150.779 & 29.532 & 58.205 & 244.482 & 12 & 16 & 184.729 \\
\hline 1987 & 68 & 156.745 & 29.789 & 64.458 & 256.610 & 23 & 16 & 192.933 \\
\hline 1988 & 70 & 162.363 & 29.834 & 72.541 & 271.710 & 23 & 16 & 207.479 \\
\hline 1989 & 75 & 169.335 & 29.510 & 87.172 & 293.204 & 20 & 21 & 222.311 \\
\hline 1990 & 75 & 183.480 & 27.451 & 104.238 & 315.169 & 25 & 21 & 239.082 \\
\hline
\end{tabular}

Quelle: Bildungsministerium (Hrsg.) 2008: Educational Statistics of the Republic of China 2008 S.4-7 und 20-27.

Anzahl der Schüler mit fünfjährigen, dreijährigen und zweijährigen Studien in den Fachschulen sind eigene Berechung anhand von S.24 und

26. 
Tabelle 11

Entwicklung der Einschulungsrate zur Sekundarstufe II, der Anzahl der Schulen, Schulklasse und Schüler sowie der durchschnittlichen Anzahl der Klassen pro Schule und der Klassenstärke in den Junior High Schools von 1968 bis 1990

\begin{tabular}{|c|c|c|c|c|c|c|}
\hline Jahr & $\begin{array}{c}\text { Einschulungsrate } \\
\text { In: } \%\end{array}$ & $\begin{array}{c}\text { Anzahl } \\
\text { der } \\
\text { Schule } \\
\end{array}$ & $\begin{array}{c}\text { Anzahl } \\
\text { der } \\
\text { Klassen }\end{array}$ & $\begin{array}{l}\text { Anzahl der } \\
\text { Schüler }\end{array}$ & $\begin{array}{c}\text { Durchschnittliche } \\
\text { Anzahl der Klassen } \\
\text { pro Schule }\end{array}$ & $\begin{array}{c}\text { Durchschnittliche } \\
\text { Klassestärke }\end{array}$ \\
\hline 1968 & 74,17 & 487 & 11.459 & 617.225 & 23,53 & 53,86 \\
\hline 1969 & 75,53 & 525 & 13.395 & 710.818 & 25,51 & 53,07 \\
\hline 1970 & 78,59 & 553 & 15.281 & 799.223 & 27,63 & 52,30 \\
\hline 1971 & 80,85 & 567 & 16.345 & 847.660 & 28,83 & 51,86 \\
\hline 1972 & 83,31 & 577 & 17.407 & 908.615 & 30,17 & 52,20 \\
\hline 1973 & 83,71 & 586 & 18.080 & 948.872 & 30,85 & 52,48 \\
\hline 1974 & 88,03 & 594 & 19.014 & 990.931 & 32,01 & 52,12 \\
\hline 1975 & 89,47 & 605 & 19.908 & 1.036 .357 & 32,91 & 52,06 \\
\hline 1976 & 90,41 & 614 & 20.454 & 1.058 .870 & 33,31 & 51.77 \\
\hline 1977 & 93,60 & 624 & 21.063 & 1.075 .455 & 33,76 & 51,06 \\
\hline 1978 & 94,08 & 632 & 21.799 & $1.082,074$ & 34,49 & 49,64 \\
\hline 1979 & 95,58 & 637 & 22.433 & 1.082 .970 & 35,22 & 48,28 \\
\hline 1980 & 96,14 & 648 & 22.811 & 1.075 .532 & 35,20 & 47,15 \\
\hline 1981 & 96,77 & 658 & 22.946 & 1.070 .942 & 34,87 & 46,67 \\
\hline 1982 & 97,96 & 661 & 23.459 & $1.082,358$ & 35,49 & 46,14 \\
\hline 1983 & 97,95 & 669 & 23.816 & $1.087,601$ & 35,60 & 45,67 \\
\hline 1984 & 98,70 & 669 & 23.768 & 1.077 .732 & 35,53 & 45,34 \\
\hline 1985 & 98,73 & 675 & 23.498 & 1.062 .226 & 34,81 & 45,20 \\
\hline 1986 & 99,04 & 676 & 23.352 & 1.052 .993 & 34,54 & 45,09 \\
\hline 1987 & 99,51 & 679 & 23.639 & 1.053 .923 & 34,81 & 44,58 \\
\hline 1988 & 99,09 & 683 & 24.573 & 1.088 .890 & 35,98 & 44,31 \\
\hline 1989 & 99,62 & 691 & 25.514 & 1.125 .238 & 36,92 & 44,10 \\
\hline 1990 & 99,77 & 700 & 26.297 & 1.160 .180 & 37,57 & 44,12 \\
\hline
\end{tabular}

Quelle: Bildungsmisteriumg (Hrsg.) 2008: Educational Statistics of the Republic of China 2008

S.4, 6, 16, 18, 20, 22 und 35

Durchschnittliche Anzahl der Klassen und durchschnittliche Klassenstärke sind eigene Berechnung. 


\begin{tabular}{|c|c|c|c|c|c|c|c|c|}
\hline \multirow[b]{3}{*}{ Jahr } & \multicolumn{8}{|c|}{$\begin{array}{l}\text { Tabelle } 12 \\
\text { Schüler an den Senior High Schools, Senior Vocational High Schools } \\
\text { Fachschulen von } 1968 \text { bis } 1990\end{array}$} \\
\hline & \multicolumn{2}{|c|}{ Senior High Schools } & \multicolumn{4}{|c|}{ Berufsbildende Schulen } & \multirow{2}{*}{\multicolumn{2}{|c|}{$\begin{array}{l}\text { Das Verhältnis der } \\
\text { Schüler } \\
\text { zwischen Senior High } \\
\text { Schools und den } \\
\text { Berufsschulen }\end{array}$}} \\
\hline & $\begin{array}{c}\text { Anzahl der } \\
\text { Schulen }\end{array}$ & $\begin{array}{l}\text { Anzahl der } \\
\text { Schüler }\end{array}$ & $\begin{array}{l}\text { Anzahl der } \\
\text { Senior } \\
\text { Vocational } \\
\text { High } \\
\text { Schools }\end{array}$ & $\begin{array}{c}\text { Anzahl der } \\
\text { Schüler in } \\
\text { den Senior } \\
\text { Vocational } \\
\text { High Schools }\end{array}$ & $\begin{array}{l}\text { Anzahl } \\
\text { der } \\
\text { Fachschulen }\end{array}$ & $\begin{array}{c}\text { Anzahl } \\
\text { der } \\
\text { Schüler in den } \\
\text { fünfjährigen } \\
\text { Fachschulen }\end{array}$ & & \\
\hline 1968 & 177 & 152.877 & 134 & 116.206 & 63 & 53.663 & 56,81 & 43,19 \\
\hline 1969 & 177 & 161.459 & 141 & 137.565 & 69 & 67.831 & 54,00 & 46,00 \\
\hline 1970 & 185 & 178.537 & 146 & 175.650 & 70 & 78.657 & 50,41 & 49,59 \\
\hline 1971 & 199 & 190.798 & 168 & 198.969 & 73 & 85.041 & 48,95 & 51,05 \\
\hline 1972 & 203 & 197.151 & 171 & 216.905 & 76 & 89.745 & 47,61 & 52,39 \\
\hline 1973 & 202 & 191.388 & 171 & 232.574 & 76 & 93.240 & 45,14 & 54,86 \\
\hline 1974 & 201 & 185.349 & 173 & 255.965 & 76 & 94.446 & 42,00 & 58,00 \\
\hline 1975 & 195 & 185.181 & 177 & 282.415 & 76 & 95.815 & 39,60 & 60,40 \\
\hline 1976 & 195 & 181.933 & 178 & 296.493 & 76 & 99.435 & 38,03 & 61,97 \\
\hline 1977 & 189 & 181.150 & 183 & 304.992 & 76 & 104.161 & 37,26 & 62,74 \\
\hline 1978 & 187 & 177.647 & 184 & 312.061 & 75 & 109.279 & 36,28 & 63,72 \\
\hline 1979 & 186 & 181.408 & 184 & 328.395 & 75 & 115.926 & 35,58 & 64,42 \\
\hline 1980 & 184 & 180.665 & 191 & 349.138 & 77 & 129.156 & 34,10 & 65,90 \\
\hline 1981 & 180 & 182.391 & 196 & 374.206 & 77 & 122.039 & 32,77 & 67,23 \\
\hline 1982 & 175 & 187.015 & 202 & 394.258 & 77 & 129.156 & 32,17 & 67,83 \\
\hline 1983 & 176 & 190.214 & 202 & 404.549 & 77 & 135.260 & 31,98 & 68,02 \\
\hline 1984 & 176 & 192.360 & 201 & 407.832 & 77 & 140.493 & 32,05 & 67,95 \\
\hline 1985 & 176 & 194.757 & 201 & 421.784 & 77 & 145.332 & 31,59 & 68,41 \\
\hline 1986 & 175 & 200.599 & 204 & 437.924 & 77 & 150.779 & 31,42 & 68,58 \\
\hline 1987 & 171 & 206.019 & 209 & 447.328 & 68 & 156.745 & 31,53 & 68,47 \\
\hline 1988 & 168 & 208.994 & 212 & 444.232 & 70 & 162.363 & 31,99 & 68,01 \\
\hline 1989 & 168 & 204.457 & 214 & 438.140 & 75 & 169.335 & 31,82 & 68,18 \\
\hline 1990 & 170 & 209.010 & 216 & 449.111 & 75 & 183.480 & 31,76 & 68,24 \\
\hline
\end{tabular}

Quelle: Bildungsministerium (Hrsg.) 2008: Educational Statistics of the Republic of China 2008

S. 4, 6, 20, 22 und 34.

Es fehlte die Angabe für die Anzahl der Schüler in den ersten drei Jahren in der Fachschule in „Educational Statistics of the Republic of China 2008“ 


\begin{tabular}{|c|c|c|c|c|c|c|c|c|}
\hline \multicolumn{9}{|c|}{$\begin{array}{l}\text { Tabelle 13 } \\
\text { Entwicklung der Schülerzahlen in unterschiedlichen Lehrgängen } \\
\text { in den Senior Vocational High Schools von } 1968 \text { bis } 1990\end{array}$} \\
\hline Jahr & Gesamt & $\begin{array}{c}\text { Agrarwirt- } \\
\text { schaft }\end{array}$ & Gewerbe & Handel & Meerwesen & $\begin{array}{l}\text { Medizin und } \\
\text { Pflege }\end{array}$ & $\begin{array}{c}\text { Haus- } \\
\text { wirtschaft }\end{array}$ & Übrige \\
\hline 1968 & 150.131 & 24.431 & 31.877 & 75.922 & 4.745 & 3.717 & 9.439 & 0 \\
\hline 1969 & 155.870 & 21.101 & 43.488 & 74.857 & 4.704 & 3.726 & 7.994 & 0 \\
\hline 1970 & 175.905 & 19.231 & 63.084 & 76.748 & 4.937 & 5.193 & 6.712 & 0 \\
\hline 1971 & 198.779 & 16.651 & 80.527 & 84.241 & 5.421 & 5.870 & 6.069 & 0 \\
\hline 1972 & 216.587 & 13.923 & 93.260 & 91.102 & 5.731 & 6.770 & 5.801 & 0 \\
\hline 1973 & 232.233 & 12.694 & 103.557 & 97.030 & 5.826 & 7.693 & 5.433 & 0 \\
\hline 1974 & 255.536 & 12.697 & 116.640 & 106.164 & 6.098 & 8.558 & 5.379 & 0 \\
\hline 1975 & 282.415 & 13.218 & 127.698 & 120.579 & 6.416 & 9.287 & 5.148 & 69 \\
\hline 1976 & 295.762 & 14.045 & 133.404 & 126.829 & 6.089 & 9.670 & 5.490 & 235 \\
\hline 1977 & 304.198 & 14.521 & 135.852 & 130.785 & 6.409 & 9.979 & 6.310 & 342 \\
\hline 1978 & 311.294 & 14.098 & 140.188 & 131.483 & 6.557 & 9.536 & 8.589 & 843 \\
\hline 1979 & 327.559 & 14.538 & 149.150 & 137.730 & 6.623 & 10.376 & 8.100 & 1.042 \\
\hline 1980 & 349.138 & 15.191 & 163.982 & 140.615 & 6.252 & 11.000 & 10.911 & 1.187 \\
\hline 1981 & 373.142 & 16.334 & 180.908 & 146.076 & 5.745 & 11.073 & 12.514 & 419 \\
\hline 1982 & 393.022 & 16.809 & 194.550 & 149.113 & 6.038 & 11.389 & 14.586 & 537 \\
\hline 1983 & 403.185 & 17.411 & 201.126 & 149.110 & 6.085 & 11.620 & 17.280 & 553 \\
\hline 1984 & 407.832 & 17.874 & 203.121 & 148.465 & 6.067 & 11.836 & 19.889 & 580 \\
\hline 1985 & 421.784 & 18.376 & 209.701 & 149.833 & 6.022 & 12.196 & 25.048 & 608 \\
\hline 1986 & 437.924 & 19.132 & 215.746 & 150.970 & 6.111 & 12.496 & 32.772 & 697 \\
\hline 1987 & 447.328 & 19.834 & 217.912 & 149.905 & 5.960 & 12.770 & 40.142 & 805 \\
\hline 1988 & 444.232 & 19.703 & 211.007 & 148.725 & 5.707 & 12.926 & 45.106 & 1.058 \\
\hline 1989 & 438.140 & 18.327 & 215.508 & 151.849 & 5.341 & 14.009 & 31.841 & 1.265 \\
\hline 1990 & 449.111 & 17.801 & 202.978 & 159.119 & 5.677 & 15.664 & 46.169 & 1.703 \\
\hline
\end{tabular}

Quelle: CEPD (Hrsg.) 1995 und 2007: Taiwan Statistical Data Book 1995 S.269 und ders. 2007 S.284.

\begin{tabular}{|c|c|c|c|c|c|c|c|c|}
\hline \multicolumn{10}{|c|}{$\begin{array}{l}\text { Tabelle 13 a } \\
\text { Entwicklung der Schüleranzahl und Anteil der Schüler in unterschiedlichen Lehrgängen } \\
\text { in der Senior Vocational High School in den Jahren 1968, 1978 und 1986 }\end{array}$} \\
\hline & Gesamt & $\begin{array}{c}\text { Agrarwirt- } \\
\text { schaft }\end{array}$ & Gewerbe & Handel & $\begin{array}{c}\text { Meerwese } \\
\mathrm{n}\end{array}$ & $\begin{array}{c}\text { Medizin und } \\
\text { Pflege }\end{array}$ & $\begin{array}{c}\text { Hauswirtscha } \\
\mathrm{ft}\end{array}$ & Übrige \\
\hline 1968 & 150.131 & 24.431 & 31.877 & 75.922 & 4.745 & 3.717 & 9.439 & 0 \\
\hline Anteil in \% & 100 & 16,27 & 21,23 & 50,57 & 3,16 & 2,48 & 6,29 & \\
\hline 1978 & 311.294 & 14.098 & 140.188 & 131.483 & 6.557 & 9.536 & 8.589 & 843 \\
\hline Anteil in \% & 100 & 4,53 & 45,03 & 42,24 & 2,11 & 3,06 & 2,76 & 0,27 \\
\hline 1986 & 437.924 & 19.132 & 215.746 & 150.970 & 6.111 & 12.496 & 32.772 & 697 \\
\hline Anteil in \% & 100 & 4,37 & 49,27 & 34,47 & 1,40 & 2,85 & 7,48 & 0,16 \\
\hline
\end{tabular}

Anteil der Schüler ist eigene Berechnung 
Tabelle 14

Entwicklung der Anzahl der Schulen und Schüler in den gesamten und privaten Bildungseinrichtungen für Senior High Schools und Senior Vocational High Schools von 1968 bis 1990

\begin{tabular}{|c|c|c|c|}
\hline & Senior High Schools & $\begin{array}{c}\text { Senior } \\
\text { Vocational High Schools }\end{array}$ \\
\hline
\end{tabular}

\begin{tabular}{|c|c|c|c|c|c|}
\hline Jahr & & Schülerzahl & Schulzahl & Schülerzahl & Schulzahl \\
\hline 1968 & gesamte & 152.877 & 177 & 116.206 & 134 \\
\hline & private & 35.643 & 98 & 50.831 & 55 \\
\hline 1969 & gesamte & 161.459 & 177 & 137.642 & 141 \\
\hline
\end{tabular}

\begin{tabular}{|c|c|c|c|c|c|}
\hline & gesamile & 101.459 & $17 /$ & שדט. & \\
\hline & private & 36.866 & 95 & 63.702 & 63 \\
\hline \multirow[t]{2}{*}{1970} & gesamte & 178.537 & 185 & 175.650 & 146 \\
\hline & private & 43.876 & 104 & 89.154 & 70 \\
\hline 1971 & gesamte & 190.798 & 199 & 198.969 & 168 \\
\hline
\end{tabular}

\begin{tabular}{|c|c|c|c|c|c|}
\hline 1971 & gesamte & 190.798 & 199 & 198.969 & 168 \\
\hline & private & 46.559 & 118 & 104.859 & 92 \\
\hline 1972 & gesamte & 197.151 & 203 & 216.905 & 171 \\
\hline & private & 47.315 & 121 & 116.320 & 97 \\
\hline 1973 & gesamte & 191.388 & 202 & 232.574 & 171 \\
\hline & private & 43.064 & 120 & 128.270 & 96 \\
\hline 1974 & gesamte & 185.349 & 201 & 255.965 & 173 \\
\hline
\end{tabular}

\begin{tabular}{|l|c|c|c|c|c|}
\hline & private & 36.484 & 113 & 170.792 & 102 \\
\hline 1977 & gesamte & 181.150 & 189 & 1704.992 & 183 \\
\hline & private & 36.763 & 107 & 312.061 & 184 \\
\hline 1978 & gesamte & 177.647 & 187 & 180.976 & 106 \\
\hline & private & 35.552 & 105 & 328.395 & 184 \\
\hline 1979 & gesamte & 181.408 & 186 & 191.680 & 106 \\
\hline & private & 38.834 & 104 & 349.138 & 191 \\
\hline 1980 & gesamte & 180.665 & 184 & 205.634 & 110 \\
\hline & private & 39.008 & 101 & 374.206 & 196 \\
\hline 1981 & gesamte & 182.391 & 180 & 220.875 & 111 \\
\hline & private & 40.763 & 100 & 394.258 & 202 \\
\hline 1982 & gesamte & 187.015 & 175 & 234.941 & 115 \\
\hline & private & 41.840 & 96 & 404.549 & 202 \\
\hline & gesamte & 190.214 & 176 & 240.286 & 115 \\
\hline & private & 43.147 & 96 & 407.832 & 201 \\
\hline & gesamte & 192.360 & 176 & 244.869 & 114 \\
\hline
\end{tabular}




\begin{tabular}{|l|c|c|c|c|c|}
\hline 1985 & gesamte & 194.757 & 176 & 421.784 & 201 \\
\hline & private & 47.340 & 96 & 255.298 & 113 \\
\hline 1986 & gesamte & 200.599 & 175 & 437.924 & 204 \\
\hline & private & 50.176 & 95 & 268.179 & 115 \\
\hline 1987 & gesamte & 206.019 & 171 & 447.328 & 209 \\
\hline & private & 51.805 & 95 & 270.144 & 115 \\
\hline 1988 & gesamte & 208.994 & 168 & 444.232 & 212 \\
\hline & private & 53.247 & 92 & 263.435 & 214 \\
\hline 1989 & gesamte & 204.457 & 168 & 438.140 & 120 \\
\hline & private & 52.993 & 90 & 259.445 & 216 \\
\hline 1990 & gesamte & 209.010 & 170 & 449.111 & 121 \\
\hline
\end{tabular}

Quelle : Bildungsminsteriumg (Hrsg.) 2009

Educational Statistics of the Republic of China 2008 S.4-7 und 20-23. 


\begin{tabular}{|c|c|c|c|c|c|c|c|c|}
\hline \multirow[b]{3}{*}{$\mathrm{Jahr}$} & \multicolumn{7}{|c|}{$\begin{array}{l}\text { Tabelle } 15 \\
\text { Imten und privaten Bildungseinrichtungen und Studenten } \\
\text { Hochschulen von } 1950 \text { bis } 1990\end{array}$} & \\
\hline & & \multicolumn{3}{|c|}{ Anzahl an der Bildungseinrichtungen } & \multicolumn{4}{|c|}{ Anzahl der Studenten } \\
\hline & & Fachschulen & Fachhochschulen & Universitäten & Fachstudien & \begin{tabular}{|c|} 
Bachlor- \\
studien
\end{tabular} & $\begin{array}{l}\text { Magister- } \\
\text { Studien }\end{array}$ & Promotion \\
\hline \multirow[t]{2}{*}{1950} & gesamte & 3 & 3 & 1 & 1.286 & 5.374 & 5 & \\
\hline & private & 1 & - & - & 374 & - & - & - \\
\hline \multirow[t]{2}{*}{1951} & gesamte & 4 & 3 & 1 & 2.140 & 6.057 & 12 & - \\
\hline & private & 1 & - & - & 651 & - & - & - \\
\hline \multirow[t]{2}{*}{1952} & gesamte & 4 & 3 & 1 & 3.171 & 6.853 & 13 & - \\
\hline & private & 1 & - & - & 893 & - & - & - \\
\hline \multirow[t]{2}{*}{1953} & gesamte & 5 & 3 & 1 & 4.240 & 7.687 & 16 & - \\
\hline & private & 1 & - & - & 954 & - & - & - \\
\hline \multirow[t]{2}{*}{1954} & gesamte & 7 & 5 & 2 & 4.587 & 9.001 & 82 & - \\
\hline & private & 1 & 2 & - & 821 & 461 & - & - \\
\hline \multirow[t]{2}{*}{1955} & gesamte & 5 & 6 & 4 & 4.545 & 13.460 & 169 & - \\
\hline & private & 1 & 3 & 1 & 992 & 1.455 & - & - \\
\hline \multirow[t]{2}{*}{1956} & gesamte & 6 & 5 & 6 & 5.025 & 17.309 & 271 & 1 \\
\hline & private & 2 & 3 & 1 & 1.808 & 2.212 & - & - \\
\hline \multirow[t]{2}{*}{1957} & gesamte & 6 & 5 & 6 & 4.879 & 20.394 & 346 & - \\
\hline & private & 2 & 3 & 1 & 1.827 & 3.329 & - & - \\
\hline \multirow[t]{2}{*}{1958} & gesamte & 7 & 7 & 7 & 5.822 & 21.739 & 373 & 4 \\
\hline & private & 3 & 5 & 1 & 2.079 & 4.190 & - & - \\
\hline \multirow[t]{2}{*}{1959} & gesamte & 8 & 7 & 7 & 5.624 & 23.709 & 427 & 10 \\
\hline & private & 3 & 5 & 1 & 1.469 & 5.486 & - & - \\
\hline \multirow[t]{2}{*}{1960} & gesamte & 12 & 8 & 7 & 7.888 & 26.735 & 426 & 11 \\
\hline & private & 6 & 6 & 1 & 2.854 & 6.607 & - & - \\
\hline \multirow[t]{2}{*}{1961} & gesamte & 14 & 8 & 8 & 8.366 & 29.524 & 501 & 12 \\
\hline & private & 6 & 8 & 1 & 3.378 & 7.313 & 8 & - \\
\hline \multirow[t]{2}{*}{1962} & gesamte & 15 & 9 & 9 & 11.074 & 32.532 & 693 & 15 \\
\hline & private & 6 & 9 & 1 & 4.990 & 8.392 & 90 & - \\
\hline \multirow[t]{2}{*}{1963} & gesamte & 15 & 10 & 10 & 13.171 & 37.719 & 801 & 16 \\
\hline & private & 4 & 10 & 2 & 5.660 & 12.367 & 172 & - \\
\hline \multirow[t]{2}{*}{1964} & gesamte & 20 & 11 & 10 & 17.923 & 45.203 & 866 & 18 \\
\hline & private & 8 & 10 & 2 & 8.742 & 17.981 & 177 & - \\
\hline \multirow[t]{2}{*}{1965} & gesamte & 35 & 11 & 10 & 29.534 & 54.819 & 974 & 19 \\
\hline & private & 20 & 10 & 2 & 18.022 & 24.838 & 159 & - \\
\hline 1966 & gesamte & 48 & 11 & 10 & 47.499 & 65.245 & 1.083 & 28 \\
\hline & private & 32 & 10 & 2 & 32.218 & 32.706 & 150 & - \\
\hline 1967 & gesamte & 57 & 13 & 9 & 63.411 & 73.881 & 1.271 & 50 \\
\hline & private & 39 & 10 & 2 & 43.804 & 38.586 & 175 & 13 \\
\hline 1968 & gesamte & 63 & 14 & 8 & 79.456 & 80.255 & 1.531 & 95 \\
\hline & private & 43 & 10 & 2 & 57.092 & 41.636 & 250 & 28 \\
\hline 1969 & gesamte & 69 & 13 & 9 & 95.988 & 86.233 & 1.856 & 138 \\
\hline & private & 49 & 9 & 3 & 70.149 & 44.883 & 321 & 43 \\
\hline 1970 & gesamte & 70 & 13 & 9 & 108.328 & 92.850 & 2.129 & 166 \\
\hline & private & 50 & 9 & 3 & 79.882 & 48.684 & 330 & 43 \\
\hline 1971 & gesamte & 73 & 14 & 9 & 119.146 & 100.455 & 2.697 & 207 \\
\hline & private & 53 & 9 & 3 & 87.333 & \begin{tabular}{|l|}
52.931 \\
\end{tabular} & 411 & 56 \\
\hline 1972 & gesamte & 76 & 14 & 9 & 138.310 & 109.827 & 2.693 & 228 \\
\hline & private & 56 & 9 & 3 & 95.943 & 59.577 & 534 & 57 \\
\hline
\end{tabular}




\begin{tabular}{|c|c|c|c|c|c|c|c|c|}
\hline 1973 & gesamte & 76 & 14 & 9 & 147.621 & 120.304 & 2.745 & 225 \\
\hline & private & 56 & 9 & 3 & 104.291 & 67.688 & 608 & 53 \\
\hline \multirow[t]{2}{*}{1974} & gesamte & 76 & 15 & 9 & 149.917 & 128.930 & 3.042 & 279 \\
\hline & private & 56 & 9 & 3 & 108.423 & 73.625 & 754 & 94 \\
\hline \multirow[t]{2}{*}{1975} & gesamte & 76 & 16 & 9 & 150.226 & 135.297 & 3.614 & 298 \\
\hline & private & 56 & 9 & 3 & 110.527 & 78.866 & 948 & 99 \\
\hline \multirow[t]{2}{*}{1976} & gesamte & 76 & 16 & 9 & 154.056 & 140.857 & 4.138 & 363 \\
\hline & private & 56 & 9 & 3 & 115.899 & 82.512 & 1.074 & 103 \\
\hline \multirow[t]{2}{*}{1977} & gesamte & 76 & 17 & 9 & 160.506 & 142.921 & 4.749 & 407 \\
\hline & private & 56 & 10 & 3 & 121.876 & 84.047 & 1.252 & 120 \\
\hline \multirow[t]{2}{*}{1978} & gesamte & 75 & 17 & 9 & 166.535 & 145.210 & 4.974 & 469 \\
\hline & private & 55 & 10 & 3 & 127.027 & 85.678 & 1.297 & 143 \\
\hline \multirow[t]{2}{*}{1979} & gesamte & 75 & 15 & 11 & 174.623 & 149.370 & 5.049 & 561 \\
\hline & private & 55 & 10 & 3 & 134.317 & 88.758 & 1.288 & 166 \\
\hline \multirow[t]{2}{*}{1980} & gesamte & 77 & 11 & 16 & 183.134 & 153.088 & 5.633 & 673 \\
\hline & private & 56 & 6 & 7 & 140.292 & 91.285 & 1.471 & 185 \\
\hline \multirow[t]{2}{*}{1981} & gesamte & 77 & 11 & 16 & 192.901 & 158.181 & 6.555 & 800 \\
\hline & \begin{tabular}{|l|} 
private \\
\end{tabular} & 56 & 6 & 7 & 147.348 & 95.466 & 1.733 & 212 \\
\hline \multirow[t]{2}{*}{1982} & gesamte & 77 & 6 & 16 & 203.722 & 163.482 & 7.517 & 975 \\
\hline & private & 56 & 6 & 7 & 155.706 & 99.772 & 2.048 & 243 \\
\hline \multirow[t]{2}{*}{1983} & gesamte & 77 & 6 & 16 & 216.165 & 169.341 & 8.427 & 1.220 \\
\hline & private & 56 & 6 & 7 & 165.046 & 103.910 & 2.292 & 270 \\
\hline \multirow[t]{2}{*}{1984} & gesamte & 77 & 6 & 16 & 227.492 & 173.908 & 9.481 & 1.500 \\
\hline & private & 56 & 6 & 7 & 173.120 & 108.051 & 2.601 & 315 \\
\hline \multirow[t]{2}{*}{1985} & gesamte & 77 & 12 & 16 & 236.824 & 179.334 & 10.638 & 1.780 \\
\hline & private & 56 & 6 & 7 & 181.178 & 111.505 & 2.870 & 327 \\
\hline \multirow[t]{2}{*}{1986} & gesamte & 77 & 12 & 16 & 244.482 & 184.729 & 11.294 & 2.143 \\
\hline & private & 56 & 6 & 7 & 190.946 & 115.478 & 3.056 & 356 \\
\hline \multirow[t]{2}{*}{1987} & gesamte & 68 & 23 & 16 & 256.610 & 192.933 & 12.426 & 2.695 \\
\hline & private & 56 & 7 & 7 & 203.894 & 119.698 & 3.241 & 381 \\
\hline \multirow[t]{2}{*}{1988} & gesamte & 70 & 23 & 16 & 271.710 & 207.479 & 14.119 & 3.222 \\
\hline & private & 58 & 7 & 7 & 221.886 & 125.375 & 3.571 & 441 \\
\hline \multirow[t]{2}{*}{1989} & gesamte & 75 & 20 & 21 & 293.204 & 222.311 & 15.750 & 3.799 \\
\hline & private & 62 & 7 & 8 & 243.690 & 131,424 & 3.902 & 494 \\
\hline \multirow[t]{2}{*}{1990} & gesamte & 75 & 25 & 21 & 315.169 & 239.082 & 17.935 & 4.437 \\
\hline & private & 62 & 12 & 8 & 264.500 & 139.709 & 4.431 & 541 \\
\hline
\end{tabular}

Quelle: Bildungsministerium (Hrsg.) 2008: Educational Statistics of the Republic of China 2008 S.4-7 und $20-23$. 


\begin{tabular}{|c|c|c|c|c|c|c|c|}
\hline \multicolumn{8}{|c|}{$\begin{array}{c}\text { Tabelle } 16 \\
\text { Wandel der Anazahl der Prüfungsanmeldungen und der aufgenommenen S }\end{array}$} \\
\hline Jahr & Prüfungsanmeldungen & Aufgenommene & $\begin{array}{c}\text { Aufnahme- } \\
\text { rate }(\%)\end{array}$ & Jahr & Prüfungsanmeldungen & Aufgenommene & $\begin{array}{c}\text { Aufnahme- } \\
\text { rate }(\%)\end{array}$ \\
\hline 1954 & 10.532 & 2.135 & 20,27 & 1978 & 94.850 & 26.603 & 28,30 \\
\hline 1955 & 12.074 & 2.344 & 19,42 & 1979 & 94.697 & 26.847 & 29,26 \\
\hline 1956 & 17.272 & 3.193 & 18,48 & 1980 & 97.182 & 27.704 & 29,25 \\
\hline 1957 & 18.601 & 5.262 & 28,29 & 1981 & 97.963 & 29.260 & 29,87 \\
\hline 1958 & 17.789 & 6.242 & 35,09 & 1982 & 95.906 & 29.938 & 31,22 \\
\hline 1959 & 23.683 & 7.170 & 30,27 & 1983 & 96.421 & 30.803 & 31,95 \\
\hline 1960 & 26.564 & 9.400 & 35,38 & 1984 & 98.236 & 31.535 & 32,10 \\
\hline 1961 & 29.959 & 11.682 & 38,99 & 1985 & 102.004 & 32.473 & 31,84 \\
\hline 1962 & 32.492 & 12.502 & 38,48 & 1986 & 110.384 & 33.848 & 30,66 \\
\hline 1963 & 33.449 & 13.014 & 38,91 & 1987 & 108.656 & 35.651 & 32,81 \\
\hline 1964 & 36.598 & 14.407 & 39,36 & 1988 & 112.327 & 37.929 & 33,77 \\
\hline 1965 & 43.456 & 13.346 & 30,71 & 1989 & 117.845 & 40.380 & 34,27 \\
\hline 1966 & 49.141 & 18.995 & 38,53 & 1990 & 120.247 & 44.836 & 37,29 \\
\hline 1967 & 55.854 & 20.029 & 38,65 & 1991 & 122.247 & 49.012 & 40,09 \\
\hline 1968 & 58.791 & 21.145 & 35,96 & 1992 & 119.950 & 52.949 & 43,76 \\
\hline 1969 & 68.930 & 23.360 & 33,89 & 1993 & 126.126 & 55.328 & 43,87 \\
\hline 1970 & 74.437 & 25.954 & 34,60 & 1994 & 124.786 & 55.386 & 44,38 \\
\hline 1971 & 78.872 & 27.935 & 35,42 & 1995 & 125.490 & 55.604 & 44,31 \\
\hline 1972 & 83.971 & 22.676 & 26,95 & 1996 & 124.654 & 61.381 & 49,24 \\
\hline 1973 & 98.077 & 24.235 & 23,71 & 1997 & 123.545 & 74.333 & 60,17 \\
\hline 1974 & 93.205 & 25.010 & 26,83 & 1998 & 118.827 & 71.826 & 60,45 \\
\hline 1975 & 97.859 & 25.797 & 26,36 & 1999 & 121.122 & 72.471 & 59,83 \\
\hline 1976 & 94.807 & 26.197 & 27,63 & 2000 & 130.468 & 75.281 & 57,70 \\
\hline 1977 & 91.907 & 26.603 & 28,95 & 2001 & 126.233 & 77.455 & 61,36 \\
\hline
\end{tabular}

Von 1957 bis 1974

Quelle: Das vierte Jahrbuch der Bildung ROC S.732-733.

Von 1975 bis 1990

Quelle: Educational Statistics of the Republic of China 2008 S.42-44. 


\begin{tabular}{|c|c|c|c|c|c|c|c|c|c|c|c|c|c|c|c|}
\hline \multirow{4}{*}{$\begin{array}{l}\text { Jahr } \\
\\
1967\end{array}$} & \multicolumn{15}{|c|}{$\begin{array}{c}\text { Tabelle } 16 \text { a } \\
\text { Entwicklung der Prüfungsanmeldungen, der aufgenommenen Studenten un }\end{array}$} \\
\hline & \multicolumn{5}{|c|}{ Anzahl der Prüfungsanmeldungen } & \multicolumn{5}{|c|}{ Aufgenommenen Studenten } & \multicolumn{5}{|c|}{ Aufnahmerate zu den Hochschulen :in \% } \\
\hline & & \multicolumn{2}{|c|}{$\begin{array}{c}\text { Naturwissenschaften und } \\
\text { angewandte } \\
\text { Naturwissenschaften }\end{array}$} & \multicolumn{2}{|c|}{$\begin{array}{c}\text { Geistes- und } \\
\text { Sozialwissenschaften }\end{array}$} & & \multirow{2}{*}{\multicolumn{2}{|c|}{$\begin{array}{c}\begin{array}{c}\text { Naturwissenschaften } \\
\text { und angewandte } \\
\text { Naturwissenschaften }\end{array} \\
9.496\end{array}$}} & \multicolumn{2}{|c|}{$\begin{array}{c}\text { Geistes- und } \\
\text { Sozialwissenschaften }\end{array}$} & & \multicolumn{2}{|c|}{$\begin{array}{l}\text { Naturwissenschaften } \\
\text { und angewandte } \\
\text { Naturwissenschaften }\end{array}$} & \multicolumn{2}{|c|}{$\begin{array}{c}\text { Geistes- und } \\
\text { Sozialwissenschaften }\end{array}$} \\
\hline & 55.854 & & & & & 19.979 & & & & & 35,77 & & & & \\
\hline 1968 & 58.791 & & & & & 21.145 & \multicolumn{2}{|c|}{10.236} & \multicolumn{2}{|c|}{10.909} & 35,97 & & & \multicolumn{2}{|c|}{51,59} \\
\hline 1969 & 68.930 & & & & & 23.326 & \multicolumn{2}{|c|}{11.304} & \multicolumn{2}{|c|}{12.022} & 33,84 & & & \multicolumn{2}{|c|}{51,54} \\
\hline 1970 & 74.437 & & & & & 25.902 & \multicolumn{2}{|c|}{12.203} & \multicolumn{2}{|c|}{13.699} & 34,79 & & & \multicolumn{2}{|c|}{52,89} \\
\hline 1971 & 78.872 & & & & & 27.935 & \multicolumn{2}{|c|}{13.352} & \multicolumn{2}{|c|}{14.483} & 35,42 & & & \multicolumn{2}{|c|}{53,20} \\
\hline 1972 & 83.971 & & & & & 22.633 & \multicolumn{2}{|c|}{12.193} & \multicolumn{2}{|c|}{10.440} & 26,95 & & & \multicolumn{2}{|c|}{46,13} \\
\hline 1973 & 98.077 & & & & & 24.235 & \multicolumn{2}{|c|}{13.126} & \multicolumn{2}{|c|}{11.109} & 23,71 & & & \multicolumn{2}{|c|}{45,84} \\
\hline 1974 & 93.205 & & & & & 25.010 & \multicolumn{2}{|c|}{13.741} & \multicolumn{2}{|c|}{11.269} & 26,83 & & & & \\
\hline 1975 & 97.859 & 25.499 & 10.560 & 33.466 & 28.334 & 25.797 & 10.348 & 4.195 & 5.012 & 6.242 & 26,36 & 40,58 & 39,73 & 14,98 & 22,03 \\
\hline 1976 & 94.807 & 27.506 & 11.726 & 26.326 & 29.249 & 26.197 & 10.715 & 4.273 & 4.927 & 6.282 & 27,63 & 38,96 & 36,44 & 18,72 & 21,48 \\
\hline 1977 & 91.907 & 28.420 & 12.612 & 25.545 & 25.330 & 26.603 & 10.850 & 4.437 & 4.979 & 6.337 & 28,95 & 38,18 & 35,18 & 19,49 & 25,02 \\
\hline 1978 & 94.850 & 29.274 & 13.745 & 26.073 & 25.758 & 26.847 & 11.124 & 4.463 & 4.942 & 6.318 & 28,30 & 38,00 & 32,47 & 18,95 & 24,53 \\
\hline 1979 & 94.697 & 29.463 & 14.015 & 25.292 & 25.927 & 27.704 & 11.355 & 4.773 & 5.078 & 6.498 & 29,26 & 38,54 & 34,06 & 20,08 & 25,06 \\
\hline 1980 & 97.182 & 30.473 & 13.264 & 26.419 & 27.026 & 28.426 & 11.520 & 4.980 & 5.213 & 6.713 & 29,25 & 37,80 & 37,55 & 19,73 & 24,84 \\
\hline 1981 & 97.963 & 30.557 & 12.170 & 28.489 & 26.747 & 29.260 & 11.748 & 5.216 & 5.253 & 7.043 & 29,87 & 38,45 & 42,86 & 18,44 & 26,33 \\
\hline 1982 & 95.906 & 30.350 & 10.722 & 26.077 & 28.757 & 29.938 & 11.992 & 5.335 & 5.328 & 7.283 & 31,22 & 39,51 & 49,76 & 20,43 & 25,33 \\
\hline 1983 & 96.421 & 30.941 & 9.017 & 27.735 & 28.728 & 30.803 & 12.014 & 5.473 & 5.655 & 7.661 & 31,95 & 38,83 & 60,70 & 20,39 & 26,67 \\
\hline
\end{tabular}

Quelle: Bildungsministerium (Hrsg.) 1974: Das vierte Jahrbuch der Bildung ROC S.732-733 und ders. Educational Statistics ROC. 1997 S.27. 